\title{
CHARACTERIZING HIGH-ENERGY-DENSITY PROPELLANTS FOR SPACE PROPULSION APPLICATIONS
}

\author{
A Dissertation
}

Presented to

The Academic Faculty

By

Timothy Kokan

In Partial Fulfillment

Of the Requirements for the Degree of

Doctor of Philosophy in the

School of Aerospace Engineering

Georgia Institute of Technology

May 2007

Copyright (C) 2007 by Timothy Kokan 


\section{CHARACTERIZING HIGH-ENERGY-DENSITY PROPELLANTS FOR SPACE PROPULSION APPLICATIONS}

Approved by:

Dr. John R. Olds, Co-chairman School of Aerospace Engineering Georgia Institute of Technology

Dr. Jerry M. Seitzman, Co-chairman School of Aerospace Engineering Georgia Institute of Technology

Dr. Peter J. Ludovice

School of Chemical and Biomolecular Engineering Georgia Institute of Technology
Dr. Mitchell L. Walker II School of Aerospace Engineering Georgia Institute of Technology

Dr. John A. Blevins Marshall Space Flight Center National Aeronautics and Space Administration 


\section{ACKNOWLEDGEMENTS}

I would like to thank everyone who has helped me throughout this research project. I would like to thank my research advisor and thesis co-chairman, Dr. John Olds, for his help and guidance throughout my graduate career at Georgia Tech. I would like to thank my thesis co-chairman, Dr. Jerry Seitzman, for his help and insight into the variety of topics covered in my thesis research. I would also like to thank the other members of my committee, Dr. Peter Ludovice, Dr. Mitchell Walker, and Dr. John Blevins, for their advice and helpful guidance.

I would like to thank my friends and coworkers in the Space Systems Design Lab. Thank you for helping to make life in Atlanta and at Georgia Tech a fun and enjoyable experience.

I would like to thank my Mom and Dad for their tireless love and support. Mom, thank you for helping me to get to this point in life. Dad, thank you for instilling in me the importance of education.

Finally, I would like to thank my wife, Stephanie, and daughter, Caroline. Stephanie, thank you for being there whenever I needed you and for sharing with me all the great experiences life has to offer. Caroline, thank you for coming into our lives and for being extra motivation to graduate! 


\section{TABLE OF CONTENTS}

ACKNOWLEDGEMENTS .................................................................................II

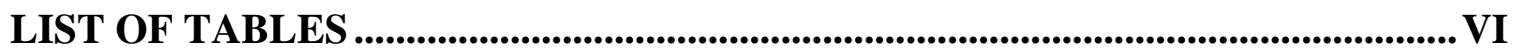

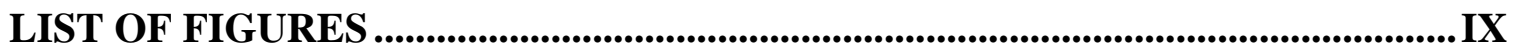

LIST OF ABBREVIATIONS ....................................................................................... XII

LIST OF ABBREVIATIONS ............................................................................................ XII

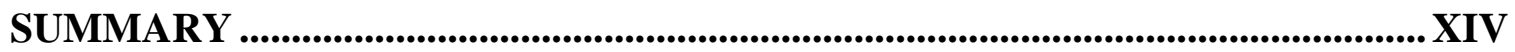

CHAPTER 1 OBJECTIVES AND MOTIVATION ................................................. 1

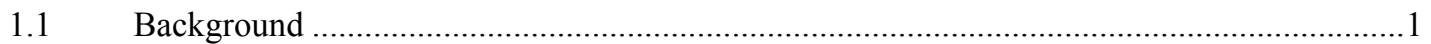

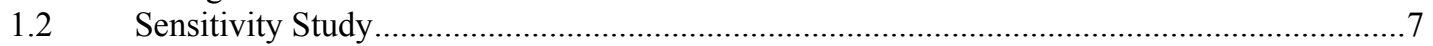

1.2.1 Overview of Conceptual Rocket Engine Powerhead Design.......................................................... 8

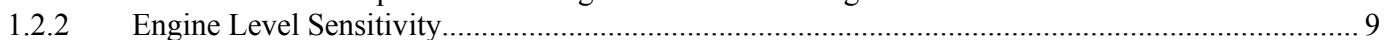

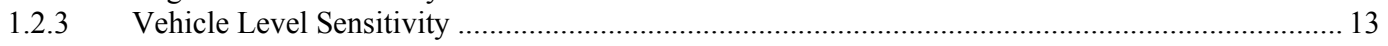

$1.3 \quad$ Unresolved Issues and Gaps in Knowledge......................................................................

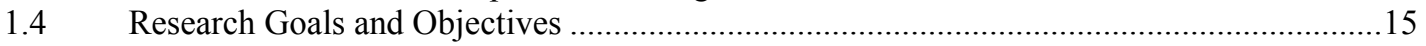

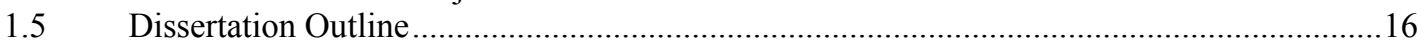

CHAPTER 2 BACKGROUND .............................................................................. 18

Evolution of Modeling Techniques to Predict Properties................................................... 18

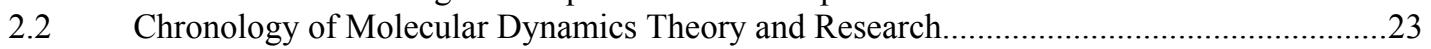

\section{CHAPTER 3 THERMOPHYSICAL PROPERTY CALCULATION METHOD... 31}

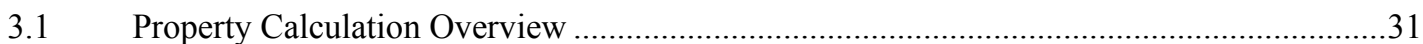

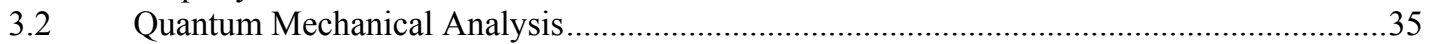

3.2.1 Determining the Minimum Energy Molecular Configuration ....................................................... 36

3.2.2 Calculation of Ideal Gas Enthalpy and Specific Heat.................................................................. 37

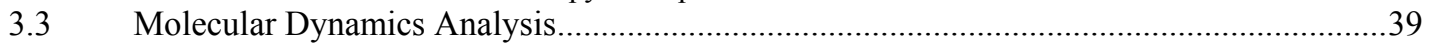

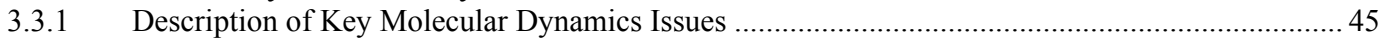

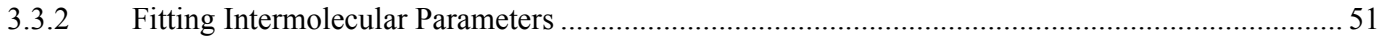

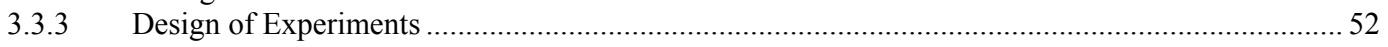

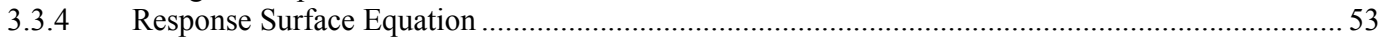

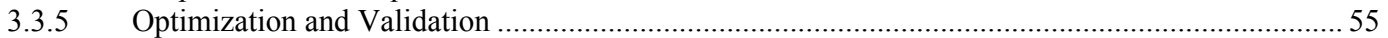

3.3.6 Molecular Dynamics Thermophysical Property Calculations ..................................................... 56

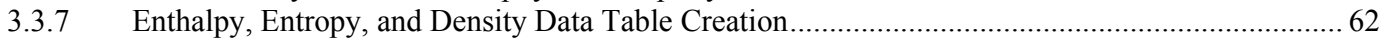

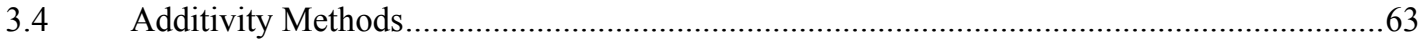

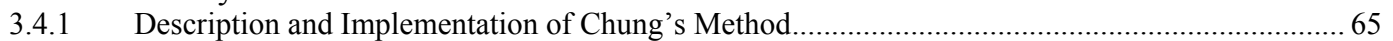

3.4.2 Creating Viscosity and Thermal Conductivity Data Tables ................................................... 70

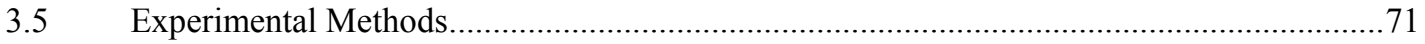

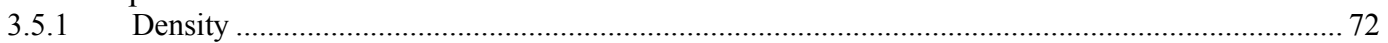

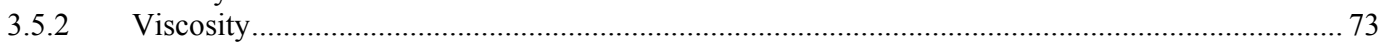

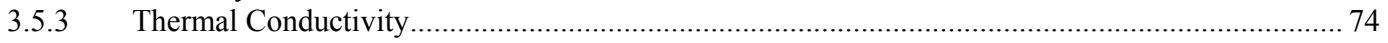

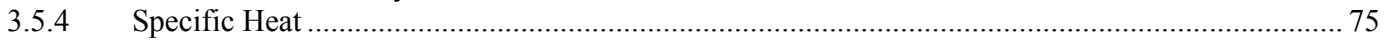

CHAPTER 4 FINAL APPLICATION .................................................................. 78

4.1 Model Compounds: Norbornane and Ethyl Azide ........................................................ 78

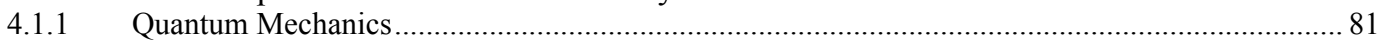

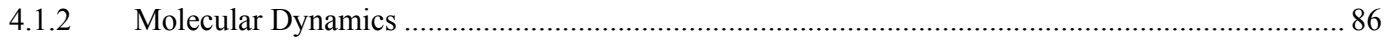

4.2 HEDM Compounds: Quadricyclane and DMAZ ................................................. 105

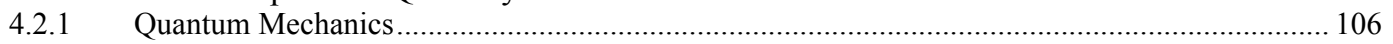




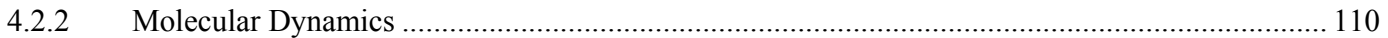

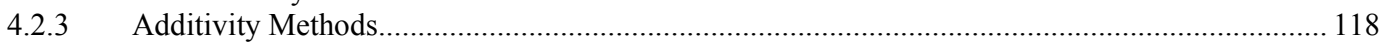

CHAPTER 5 CONCEPTUAL VEHICLE DESIGN CASE STUDY ....................... 124

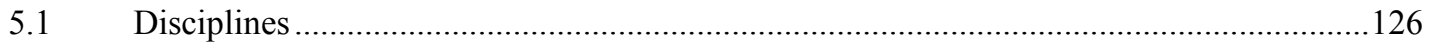

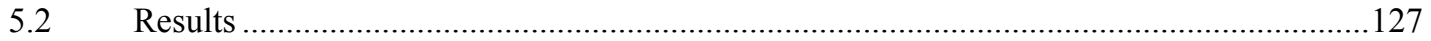

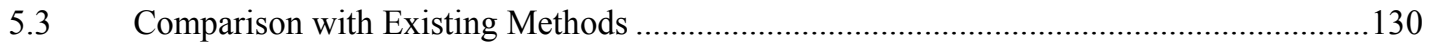

CHAPTER 6 CONCLUSIONS AND RECOMMENDATIONS ................................ 134

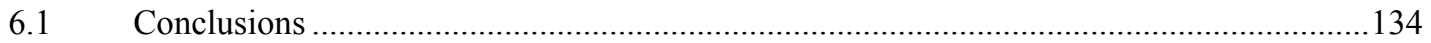

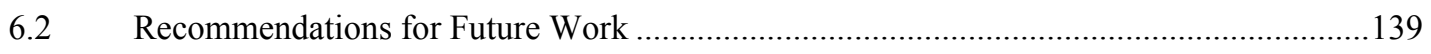

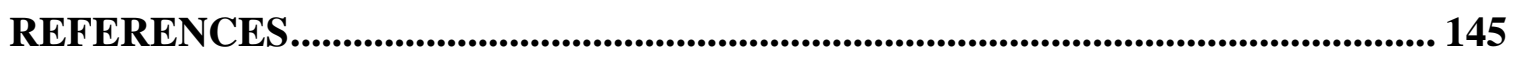

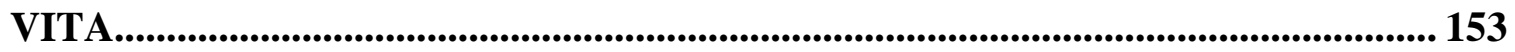




\section{LIST OF TABLES}

Table 1.1: Potential HEDM Propellants ..................................................................... 2

Table 1.2: Thermophysical Property Sensitivity Study ............................................. 12

Table 1.3: Sensitivity Study - Launch Vehicle Mass Impact ..................................... 14

Table 2.1: Bond Contributions for the Estimation of $C_{p}{ }^{o}, S^{o}$, and $\Delta h_{f}^{o}[28$, p.25] ......... 20

Table 2.2: Ring Corrections for the Estimation of $C_{p}^{o}, S^{o}$, and $\Delta h_{f}^{o}[28$, p.273] ........... 21

Table 3.1: Average RMS Deviations of MD Benzene Property Predictions.................. 44

Table 3.2: Four Design Variable CCD Run List........................................................ 53

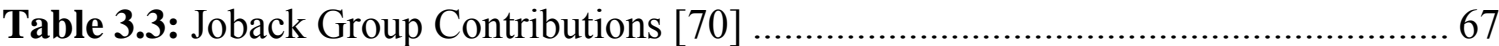

Table 3.4: Constants Used for Condensed Phase Shear Viscosity Relation [66] ........... 69

Table 3.5: Constants Used for Condensed Phase Thermal Conductivity Relation [66] .. 70

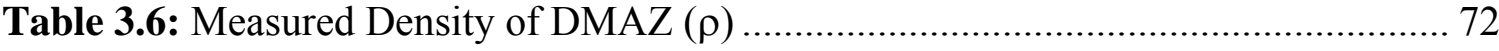

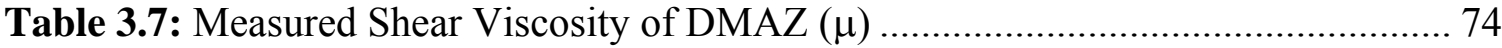

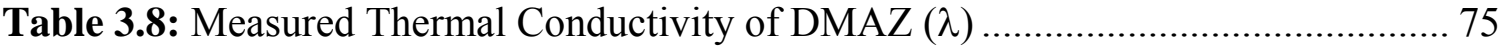

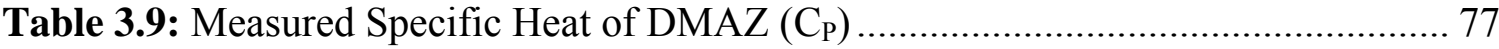

Table 4.1: Atomic Coordinates for Optimized Norbornane Geometry …...................... 82

Table 4.2: Atomic Coordinates for Optimized Ethyl Azide Geometry ......................... 82

Table 4.3: Normal Mode Vibrational Frequencies of Norbornane ............................... 84

Table 4.4: Normal Mode Vibrational Frequencies of Ethyl Azide............................... 85

Table 4.5: Ideal Gas Sensible Enthalpy Results for Norbornane................................... 86

Table 4.6: Ideal Gas Sensible Enthalpy Results for Ethyl Azide................................. 86

Table 4.7: Norbornane Baseline COMPASS Intermolecular Parameter Values ............ 87

Table 4.8: Ethyl Azide Baseline COMPASS Intermolecular Parameter Values ............ 87

Table 4.9: Norbornane Baseline COMPASS Charge Bond Increments ........................ 87

Table 4.10: Ethyl Azide Baseline COMPASS Charge Bond Increments...................... 87 
Table 4.11: Norbornane DOE - Intermolecular Parameter Multipliers and Results ....... 89

Table 4.12: Ethyl Azide DOE - Intermolecular Parameter Multipliers and Density ...... 90

Table 4.13: Norbornane DOE - Intermolecular Parameter Multipliers and $\mathrm{C}_{\mathrm{P}} \ldots \ldots \ldots \ldots . . . . .94$

Table 4.14: Ethyl Azide DOE - Intermolecular Parameter Multipliers and $C_{P} \ldots \ldots \ldots \ldots . . .95$

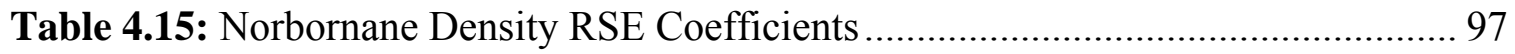

Table 4.16: Norbornane Specific Heat RSE Coefficients.......................................... 98

Table 4.17: Ethyl Azide Density RSE Coefficients .................................................. 99

Table 4.18: Ethyl Azide Specific Heat RSE Coefficients........................................ 101

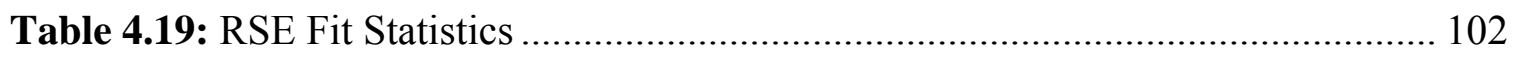

Table 4.20: Norbornane Optimized Intermolecular Parameters ................................. 103

Table 4.21: Ethyl Azide Optimized Intermolecular Parameters .................................. 104

Table 4.22: Molecular Dynamics Validation Results ............................................... 104

Table 4.23: Atomic Coordinates for Optimized Quadricyclane Geometry .................. 107

Table 4.24: Atomic Coordinates for Optimized DMAZ Geometry ............................ 107

Table 4.25: Normal Mode Vibrational Frequencies of Quadricyclane......................... 108

Table 4.26: Normal Mode Vibrational Frequencies of DMAZ ................................. 109

Table 4.27: Ideal Gas Sensible Enthalpy Results for Quadricyclane .......................... 110

Table 4.28: Ideal Gas Sensible Enthalpy Results for DMAZ .................................... 110

Table 4.29: Average RMS Deviations of Molecular Dynamics Property Predictions .. 114

Table 4.30: HEDM Compound Enthalpy and Entropy of Formation .......................... 115

Table 4.31: Quadricyclane Property Data Table Run Results .................................. 117

Table 4.32: DMAZ Property Data Table Run Results............................................... 118

Table 4.33: Additivity Method Average RMS Deviation........................................ 121

Table 4.34: Quadricyclane Additivity Method Data Table Results............................. 122

Table 4.35: DMAZ Additivity Method Data Table Results ..................................... 123

Table 5.1: ESAS Mission Parameters ........................................................................ 125

Table 5.2: Ascent Stage Engine Comparison........................................................ 128 
Table 5.3: LSAM Mass Results Comparison - MLI .............................................. 129

Table 5.4: LSAM Mass Results Comparison - MLI + Cryocooler ............................. 129

Table 5.5: Comparison of Engine Analysis Code Results ......................................... 132

Table 5.6: Comparison of Vehicle Results - MLI .................................................... 133

Table 5.7: Comparison of Vehicle Results - MLI + Cryocooler................................ 133

Table 6.1: Molecular Dynamics Average Deviations ............................................. 137

Table 6.2: Additivity Method Average Deviations................................................. 139 


\section{LIST OF FIGURES}

Figure 1.1: (a) Bond length, (b) Angle, (c) Dihedral, and (d) Out-of-Plane Motions ....... 6

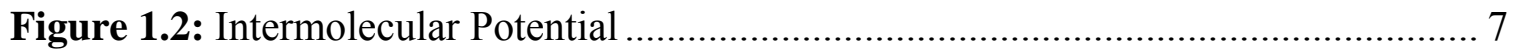

Figure 1.3: Expander Cycle Engine Diagram - Fuel Side........................................ 9

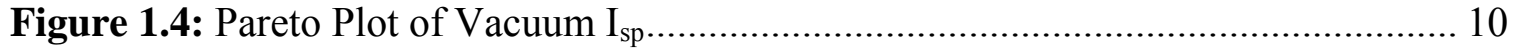

Figure 1.5: Vacuum $I_{\mathrm{sp}}$ versus Kinematic Viscosity Multiplier ................................. 11

Figure 1.6: Vacuum $I_{\mathrm{sp}}$ versus Thermal Conductivity Multiplier............................... 12

Figure 2.1: Critical Temperature-Boiling Point Relationship [26] .............................. 19

Figure 2.2: Ball and Cylinder Rendering of Chloroform $\left(\mathrm{CHCl}_{3}\right)$............................ 21

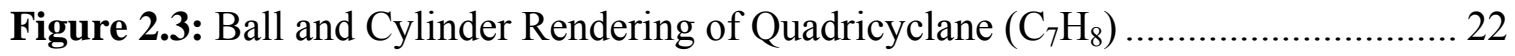

Figure 2.4: Lennard-Jones Intermolecular Potential............................................. 24

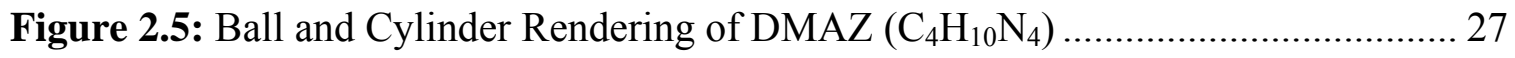

Figure 2.6: Benzene Experimental and MD Density vs. Temperature ......................... 28

Figure 2.7: Benzene Experimental and MD Enthalpy Change vs. Temperature............. 28

Figure 2.8: DMAZ Experimental and MD Density vs. Temperature ............................ 29

Figure 2.9: DMAZ Experimental and MD Enthalpy Change vs. Temperature.............. 29

Figure 3.1: Thermophysical Property Calculation Method Overview .......................... 32

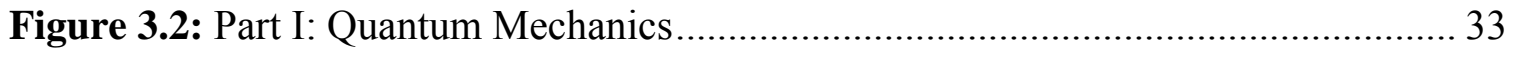

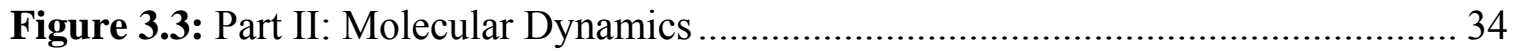

Figure 3.4: Part III: Additivity Methods ............................................................ 35

Figure 3.5: Notional Molecular Energy vs. Dihedral Angle...................................... 37

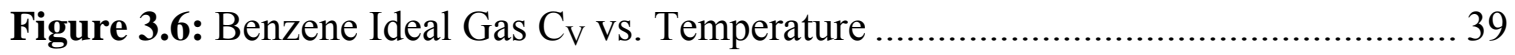

Figure 3.7: Molecular Dynamics Snapshot of Liquid DMAZ ..................................... 40

Figure 3.8: Ball and Cylinder Rendering of Quadricyclane $\left(\mathrm{C}_{7} \mathrm{H}_{8}\right) \ldots \ldots \ldots \ldots \ldots \ldots \ldots \ldots \ldots . . . . . \ldots 2$

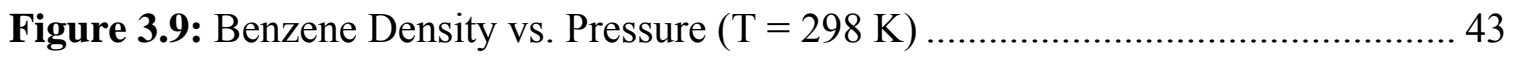

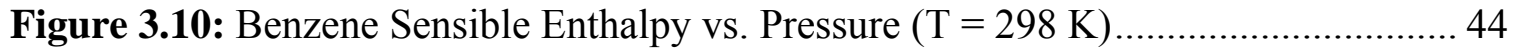


Figure 3.11: Benzene Sensible Entropy vs. Pressure $(\mathrm{T}=298 \mathrm{~K})$.............................. 44

Figure 3.12: Schematic of Periodic Boundary Conditions in Two Dimensions............. 45

Figure 3.13: Schematic of a Long-Range Cutoff in Two Dimensions .......................... 46

Figure 3.14: Benzene Molecular Dynamics Scaling Issue: T=300 K, P = $1 \mathrm{~atm} \ldots \ldots \ldots . . .48$

Figure 3.15: Density vs. Time-Step for Quadricyclane ......................................... 50

Figure 3.16: Total Energy vs. Time-Step for Quadricyclane .................................... 50

Figure 3.17: Benzene Potential Energy Interaction Issue .......................................... 59

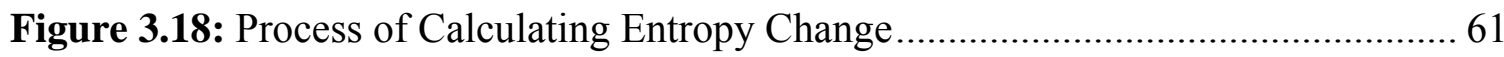

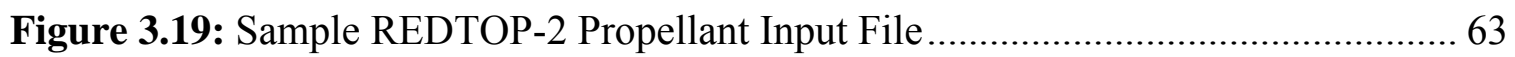

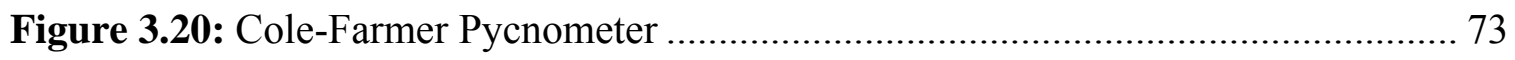

Figure 3.21: Cannon-Fenske Routine Viscometer.................................................. 74

Figure 3.22: Transient Hot-Wire Cell ................................................................... 75

Figure 3.23: TA Instruments Differential Scanning Calorimeter .............................. 76

Figure 3.24: TA Instruments Thermogravimetric Analyzer .................................... 77

Figure 4.1: Ball and Cylinder Renderings of (a) Ethyl Azide and (b) DMAZ ............... 80

Figure 4.2: Ball and Cylinder Renderings of (a) Norbornane and (b) Quadricyclane..... 80

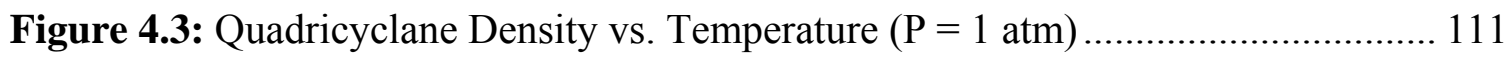

Figure 4.4: Quadricyclane Sensible Enthalpy vs. Temperature $(\mathrm{P}=1 \mathrm{~atm})$................ 112

Figure 4.5: Quadricyclane Sensible Entropy vs. Temperature $(\mathrm{P}=1 \mathrm{~atm}) \ldots \ldots \ldots \ldots \ldots \ldots . . . . . . .112$

Figure 4.6: DMAZ Density vs. Temperature ........................................................ 113

Figure 4.7: DMAZ Sensible Enthalpy vs. Temperature .......................................... 113

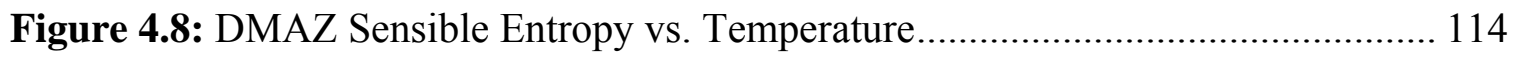

Figure 4.9: Quadricyclane Kinematic Viscosity vs. Temperature $(\mathrm{P}=1 \mathrm{~atm}) \ldots \ldots \ldots \ldots \ldots . . . . . .119$

Figure 4.10: Quadricyclane Thermal Conductivity vs. Temperature $(\mathrm{P}=1 \mathrm{~atm}) \ldots \ldots \ldots . . .119$

Figure 4.11: DMAZ Kinematic Viscosity vs. Temperature .................................... 120

Figure 4.12: DMAZ Thermal Conductivity vs. Temperature ................................... 120

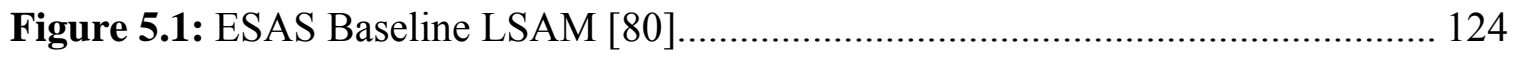


Figure 5.2: Design Structure Matrix of Conceptual Space Vehicle Design

Figure 6.1: Ball and Cylinder Rendering of (a) CPAZ and (b) MMAZ ....................... 140

Figure 6.2: Ball and Cylinder Rendering of Cubane ........................................... 140

Figure 6.3: Ball and Cylinder Rendering of Tetrapropynyl Silane............................ 141 


\section{LIST OF ABBREVIATIONS}

\begin{tabular}{|c|c|}
\hline AMBER & Assisted Model Building and Energy Refinement \\
\hline $\mathrm{CA}$ & contributing analysis \\
\hline CAD & computer-aided design \\
\hline CCD & central composite design \\
\hline CFF93 & Consistent Force Field 1993 \\
\hline CHARMM & Chemistry at Harvard Macromolecular Mechanics \\
\hline COMPASS & $\begin{array}{l}\text { Condensed-phase Optimized Molecular Potentials for Atomistic } \\
\text { Simulation Studies }\end{array}$ \\
\hline DOE & design of experiments \\
\hline DOT & Design Optimization Tool \\
\hline DMAZ & 2-azido-N, N-dimethylethanamine, $\mathrm{C}_{4} \mathrm{H}_{10} \mathrm{~N}_{4}$ \\
\hline DSC & differential scanning calorimeter \\
\hline DSM & design structure matrix \\
\hline ESAS & Exploration Systems Architecture Study \\
\hline GA & genetic algorithm \\
\hline HEDM & high-energy-density matter \\
\hline $\mathrm{HF}$ & Hartree-Fock \\
\hline $\mathrm{I}_{\mathrm{sp}}$ & specific impulse \\
\hline LAMMPS & Large-scale Atomic/Molecular Massively Parallel Simulator \\
\hline LEO & Low-Earth Orbit \\
\hline $\mathrm{LH}_{2}$ & liquid hydrogen, $\mathrm{H}_{2}$ \\
\hline LOX & liquid oxygen, $\mathrm{O}_{2}$ \\
\hline LSAM & Lunar Surface Access Module \\
\hline
\end{tabular}


MER

MLI

MM2

$\mathrm{MMH}$

NASA

NPSS

NPT

NVT

NTO

$\mathrm{O} / \mathrm{F}$

OPLS

POST

REDTOP

REDTOP-2

RMS

ROCETS

RP-1

RSE

SQP

TGA

UDMH

ZPE mass estimating relationship

multilayer insulation

Molecular Mechanics Program (Class 1)

monomethyl hydrazine, $\mathrm{CH}_{3} \mathrm{~N}_{2} \mathrm{H}_{3}$

National Aeronautics and Space Administration

Numerical Propulsion System Simulation

constant number of particles, constant pressure, constant temperature molecular dynamics simulation

constant number of particles, constant volume, constant temperature molecular dynamics simulation

nitrogen tetraoxide, $\mathrm{N}_{2} \mathrm{O}_{4}$

oxidizer-to-fuel ratio

Optimized Potentials for Liquid Simulations

Program to Optimize Simulated Trajectories

Rocket Engine Design Tool for Optimal Performance

Rocket Engine Design Tool for Optimal Performance - 2

root mean square

Rocket Engine Transient Simulation

rocket propellant-1 (kerosene)

response surface equation

sequential quadratic programming

thermogravimetric analyzer

unsymmetrical dimethylhydrazine, $\left(\mathrm{CH}_{3}\right)_{2} \mathrm{~N}_{2} \mathrm{H}_{2}$

zero-point energy 


\section{SUMMARY}

There exists wide ranging research interest in high-energy-density matter (HEDM) propellants as a potential replacement for existing industry standard fuels $\left(\mathrm{LH}_{2}\right.$, RP-1, MMH, UDMH) for liquid rocket engines. The U.S. Air Force Research Laboratory, the U.S. Army Research Lab, the NASA Marshall Space Flight Center, and the NASA Glenn Research Center each either recently concluded or currently has ongoing programs in the synthesis and development of these potential new propellants.

In order to perform conceptual designs using these new propellants, most conceptual rocket engine powerhead design tools (e.g. NPSS, ROCETS, and REDTOP-2) require several thermophysical properties of a given propellant over a wide range of temperature and pressure. These properties include enthalpy, entropy, density, viscosity, and thermal conductivity. For most of these potential new HEDM propellants, this thermophysical data either does not exist or is incomplete over the range of temperature and pressure necessary for liquid rocket engine design and analysis. Experimental testing of these properties is both expensive and time consuming and is impractical in a conceptual vehicle design environment where there is a limited amount of both time and resources.

A new technique for determining these thermophysical properties of potential new rocket engine propellants is presented. The technique uses a combination of three different computational methods to determine these properties. Quantum mechanics and molecular dynamics are used to model new propellants at a molecular level in order to calculate density, enthalpy, and entropy. Additivity methods are used to calculate the kinematic viscosity and thermal conductivity of new propellants.

By modeling the motion and distribution of the simulated molecules, molecular dynamics is used to calculate the enthalpy, entropy, and density as a function of 
temperature and pressure. Molecular dynamics simulations make use of force field equations to model the energy potential between atoms and molecules. These force field equations model the bond length stretching, bond angle bending, and dihedral angle rotating energies within a molecule as well as the electrostatic and van der Waals energies between molecules. A force field model developed by Sun in 1998, called the Condensed-phase Optimized Molecular Potentials for Atomistic Simulation Studies (COMPASS), is used as a starting point for molecular dynamics simulations of the HEDM molecules studied in this research work. The COMPASS force field model has been shown to be useful in predicting energies and densities of a variety of simple hydrocarbon molecules, but does not model more complex strained-bond hydrocarbon molecules, such as HEDM molecules, to the level of accuracy necessary for input in liquid rocket engine powerhead design codes. Modifications to the COMPASS force field model are made as part of this research work in order to improve its ability to accurately predict the required thermophysical properties over the range of pressures and temperatures experienced in a liquid rocket engine.

The COMPASS force field model modifications are made by comparing thermophysical properties predicted from molecular dynamics simulations with experimental data. The COMPASS force field model parameters are adjusted in order to minimize the difference between molecular dynamics predicted thermophysical properties and experimental data. Due to the fact that little or no experimental data exists for the HEDM compounds of interest, model compounds are used to determine the best settings for the COMPASS force field parameters. Model compounds are those compounds that have a similar molecular structure to the HEDM propellant of interest and have published thermophysical data available for use.

The new technique developed in this thesis research is validated via a series of verification experiments of HEDM compounds. Results are provided for two HEDM propellants: quadricyclane and 2-azido-N, N-dimethylethanamine (DMAZ). In each 
case, the new technique does a better job than the best current computational methods at accurately matching the experimental data of the HEDM compounds of interest.

A case study is provided to help quantify the vehicle level impacts of using HEDM propellants. The case study consists of the National Aeronautics and Space Administration's (NASA) Exploration Systems Architecture Study (ESAS) Lunar Surface Access Module (LSAM). The results of this study show that the use of HEDM propellants (either quadricyclane or DMAZ) instead of hypergolic propellants can lower the gross weight of the LSAM and may be an attractive alternative to the current baseline hypergolic propellant choice. 


\section{CHAPTER 1}

\section{OBJECTIVES AND MOTIVATION}

The primary objective of this research is the development and demonstration of a technique for predicting the thermophysical properties of new rocket propellants. The calculated thermophysical properties can be used by rocket engine powerhead analysis and design tools to predict rocket engine performance, weight, and cost, among other factors. Armed with the ability to perform powerhead designs of a rocket engine that uses the new propellants, one can then quantify the potential vehicle level impacts of the propellants.

\subsection{BACKGROUND}

HEDM propellants, as defined by the Air Force Office of Scientific Research, are propellants comprised of "advanced high energy compounds containing increased energy densities (energy to mass ratios) to produce greater specific impulses". Specific impulse, $I_{\mathrm{sp}}$, is defined with the following equation:

$$
I_{s p}=\frac{T}{g_{0} \dot{m}_{p}}
$$

In the above equation, $T$ is the total engine thrust, $g_{0}$ is the acceleration due to gravity at Earth sea-level $\left(9.81 \mathrm{~m} / \mathrm{s}^{2}\right)$, and $\dot{m}_{p}$ is the total mass flow rate of propellant. $\mathrm{I}_{\mathrm{sp}}$ is a measure of the fuel efficiency of an engine and is typically measured in units of seconds. $I_{\mathrm{sp}}$ is the number of seconds one pound weight of propellant can provide one pound force of thrust.

HEDM propellant research programs by the U.S. Air Force [1,2], U.S. Army $[3,4]$, and NASA $[5,6,7]$ have developed several promising new potential rocket 
propellants. A few selected HEDM propellants are shown in Table 1.1 with RP-1, an existing industry standard hydrocarbon fuel, listed for reference. The performance calculations are carried out at an optimal oxidizer-to-fuel, $\mathrm{O} / \mathrm{F}$, ratio to achieve the highest ideal vacuum $I_{s p}$. The $\mathrm{O} / \mathrm{F}$ ratio is the ratio of the oxidizer mass flow rate to the fuel mass flow rate in an engine. Liquid oxygen (LOX) is the oxidizer used in these performance calculations. As can be seen in this table, each of the HEDM propellants listed has a higher density and higher ideal vacuum $I_{s p}$ than that of RP-1.

Table 1.1: Potential HEDM Propellants

\begin{tabular}{ccccc}
\hline Name & $\begin{array}{c}\text { Chemical } \\
\text { Formula }\end{array}$ & $\begin{array}{c}\text { Density } \\
\left(\mathrm{g} / \mathrm{cm}^{3}\right)\end{array}$ & O/F & $\begin{array}{c}\text { Ideal Vacuum } \mathrm{I}_{s p} \\
(\mathrm{sec})^{\dagger}\end{array}$ \\
\hline RP-1 & $\mathrm{CH}_{1.942}$ & 0.80 & 2.82 & 365.1 \\
Quadricyclane & $\mathrm{C}_{7} \mathrm{H}_{8}$ & 0.99 & 2.28 & 372.5 \\
BCP & $\mathrm{C}_{6} \mathrm{H}_{8}$ & 0.85 & 2.29 & 375.9 \\
DMAZ & $\mathrm{C}_{4} \mathrm{H}_{10} \mathrm{~N}_{4}$ & 0.93 & 1.50 & 367.9 \\
1-7 Octadiyne & $\mathrm{C}_{8} \mathrm{H}_{10}$ & 0.82 & 2.32 & 373.8 \\
Cubane & $\mathrm{C}_{8} \mathrm{H}_{8}$ & 1.29 & 2.04 & 383.1 \\
\hline
\end{tabular}

${ }^{+}-I_{s p}$ calculated with LOX as oxidizer, expansion ratio $(\varepsilon)=50$, chamber pressure $\left(\mathrm{P}_{\mathrm{c}}\right)=3,000$ psia, one-dimensional equilibrium analysis, equilibrium nozzle

Although a great deal of research has been performed on HEDM propellants, knowledge about the thermophysical properties of these propellants over the range of temperature and pressure experienced in liquid rocket engines is limited. As a result, one has three main options available for designing rocket engines that utilize these propellants. The first is to perform a simple one-dimensional equilibrium analysis like the one used to compute the ideal vacuum $I_{s p}$ values found in Table 1.1. This analysis typically only requires a propellant's chemical formula, heat of formation, and density at the propellant's storage temperature and pressure. While this analysis method is considered a good preliminary step in conceptual rocket engine design, it does not provide an accurate enough prediction of engine performance $\left(I_{s p}\right)$ for most conceptual vehicle designs. Also, one-dimensional equilibrium analysis codes cannot accurately 
predict engine weight due to the fact that the various engine components, including turbopumps, preburners, and propellant valves and feed lines, are not analyzed.

The next two options can be used in conjunction with a full rocket engine powerhead analysis. The thermophysical properties required for rocket engine powerhead analyses are enthalpy, entropy, density, viscosity, and thermal conductivity as a function of temperature and pressure. The second option available is to perform laboratory measurements of all the required thermophysical properties over the range of temperature and pressure required. While this option can provide very accurate results, it is very costly and time consuming. The need for proper lab facilities, especially for extremely high temperatures and pressures, the need for expertise in using laboratory equipment, and the cost of significant amounts of experimental propellants which may be difficult to acquire and handle are all significant drawbacks to this option.

The third option is to predict the thermophysical properties of these propellants through analytical or numerical means. This option is the most appealing because one can perform the more accurate rocket engine powerhead analysis while not committing the substantial resources required to measure these properties in a laboratory.

There are many different techniques that have been used to calculate thermodynamic and physical properties of materials. Techniques ranging from new equations of state to quantum mechanics have been used to determine certain thermodynamic and physical properties. An overview of some of the most common techniques is provided in Chapter 2. A recent method developed by Sun [8] utilizes molecular dynamics simulations to calculate many thermophysical properties of alkanes and ringed hydrocarbons. Molecular dynamics is a technique of modeling the positions and velocities of molecules as a function of time. From this information, one can compute enthalpy, entropy, density, and many other thermodynamic and physical properties. 
Sun developed a new molecular force field model called COMPASS, which stands for Condensed-phase Optimized Molecular Potentials for Atomistic Simulation Studies. In molecular dynamics, the energy potential (force field) of an atom or molecule is used to model the effects of one particle on a neighboring particle. The models include the bond length stretching, bond angle bending, and dihedral angle rotating energies within a molecule, as well as the electrostatic and van der Waals forces between molecules.

The COMPASS model has been shown to be useful in predicting energies and densities of alkane and benzene compounds [8,9]. Although a variety of other force field models exist ranging from the very simple Lennard-Jones potential model [10, pp.11-12] to the more complex MM2 [11], AMBER [12,13,14,15], CHARMM [16,17], and CFF93 $[18,19]$ models, the COMPASS model is well suited for modeling hydrocarbons due to the fact that its parameters are optimized for the modeling of alkanes, alkenes, and alkynes [8]. As a result, it needs limited modifications in order to be applied to more complex strained-bond hydrocarbons. These molecular potential models are all discussed in more detail in Section 2.2 .

The COMPASS force field model has not been used to model more complex strained-bond hydrocarbons (such as those being researched by the U.S. Air Force, U.S. Army, and NASA) to the level of accuracy necessary for input in liquid rocket engine powerhead design codes. As a result, this research effort includes modifications to the COMPASS force field model in order to improve its ability to accurately predict the required thermophysical properties over the wide range of pressure and temperature experienced in a liquid rocket engine. The COMPASS force field model is shown in equation (2). 


$$
\begin{aligned}
& E^{\text {COMPASS }}=\sum_{b}\left[k_{2}\left(b-b_{0}\right)^{2}+k_{3}\left(b-b_{0}\right)^{3}+k_{4}\left(b-b_{0}\right)^{4}\right] \\
&+\sum_{\theta}\left[h_{2}\left(\theta-\theta_{0}\right)^{2}+h_{3}\left(\theta-\theta_{0}\right)^{3}+h_{4}\left(\theta-\theta_{0}\right)^{4}\right] \\
&+\sum_{\phi}\left[V_{1}(1-\cos \phi)+V_{2}(1-\cos 2 \phi)+V_{3}(1-\cos 3 \phi)\right] \\
&+\sum_{\chi} K_{\chi}\left(\chi-\chi_{0}\right)^{2} \\
&+\sum_{b, b^{\prime}} K_{b b^{\prime}}\left(b-b_{0}\right)\left(b^{\prime}-b_{0}^{\prime}\right) \\
&+\sum_{\theta, \theta^{\prime}} K_{\theta \theta^{\prime}}\left(\theta-\theta_{0}\right)\left(\theta^{\prime}-\theta_{0}^{\prime}\right) \\
&+\sum_{b, \theta} K_{b \theta}\left(b-b_{0}\right)\left(\theta-\theta_{0}\right) \\
&+\sum_{b, \phi}\left(b-b_{0}\right)\left[G_{1} \cos \phi+G_{2} \cos 2 \phi+G_{3} \cos 3 \phi\right] \\
&+\sum_{\theta, \phi}\left(\theta-\theta_{0}\right)\left[F_{1} \cos \phi+F_{2} \cos 2 \phi+F_{3} \cos 3 \phi\right] \\
&+\sum_{\theta, \theta^{\prime}, \phi} K_{\theta \theta^{\prime} \phi}\left(\theta-\theta_{0}\right)\left(\theta^{\prime}-\theta_{0}^{\prime}\right) \cos \phi \\
&+\sum_{i, j} \frac{q_{i} q_{j}}{r_{i j}} \\
&+\sum_{i, j} \varepsilon_{i j}\left[2\left(\frac{\sigma_{i j}}{r_{i j}}\right)^{9}-3\left(\frac{\sigma_{i j}}{r_{i j}}\right)^{6}\right] \text { Talence } \\
& \text { Terms } \\
& \text { Terms }
\end{aligned}
$$

The functions in the COMPASS model can be divided into two general categories: the valence terms, which represent the internal coordinates of the atoms that make up the molecule, and the nonbond interaction terms, which represent those interactions between atoms separated by two or more atoms or those that belong to different molecules [8]. The first ten terms in the equation are the valence terms while the last two terms are the nonbond interaction terms. The first four terms represent bond lengths $(b)$, bond angles $(\theta)$, dihedral angles $(\phi)$, and out-of-plane angles $(\chi)$. The out-ofplane angle, as defined by Wilson [20, p.59], is the angle between a bond connecting a central atom and its bonded atom and a plane defined by the same central atom connected to two other bonded atoms. The internal molecular motions are shown in Figure 1.1. 
The next six terms are cross-coupling terms of two or more of the internal coordinates. In the development of related molecular force field models, it has been shown that the inclusion of these cross terms is important in improving model accuracy [21]. In the work of Maple et al. [21], the inclusion of cross terms improved the model accuracy in predicting the internal energy of a formate anion by nearly $20 \%$. A sensitivity study is performed in Section 1.2 to quantify the effect of changing the thermophysical property values by $20 \%$ on engine level and vehicle level outputs.

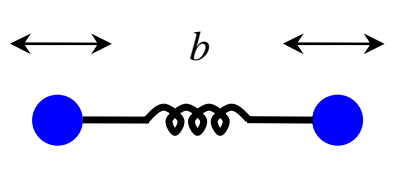

(a)

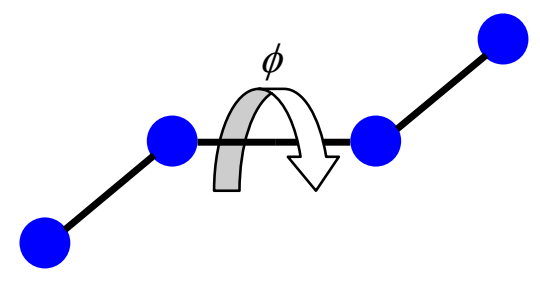

(c)

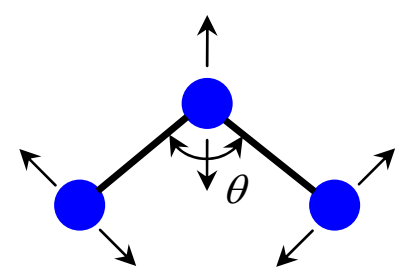

(b)

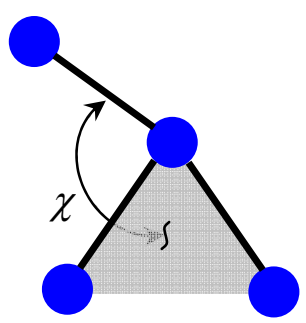

(d)

Figure 1.1: (a) Bond length, (b) Angle, (c) Dihedral, and (d) Out-of-Plane Motions

In equation (2), $k_{2}, k_{3}, k_{4}, h_{2}, h_{3}, h_{4}, V_{1}, V_{2}, V_{3}, K_{\chi}, K_{b \theta}, K_{\theta \theta}, K_{b \theta}, G_{1}, G_{2}, G_{3}, F_{1}$, $F_{2}, F_{3}$, and $K_{\theta \theta^{\prime} \phi}$ are all coefficients for the corresponding intramolecular deformations. The values of these coefficients are different for different molecules. The parameters $b_{0}$, $\theta_{0}, \phi_{0}$, and $\chi_{0}$ are the bond length, bond angle, dihedral angle, and out-of-plane angle values of the molecule in its minimum energy configuration. The parameters $b, \theta, \phi$, and $\chi$ are the bond length, bond angle, dihedral angle, and out-of-plane angle values of the molecule in its current configuration. In a molecular dynamics simulation, the values of $b, \theta, \phi$, and $\chi$ all change as a function of time. The parameters $q_{i}$ and $q_{j}$ are the charges 
on atoms $i$ and $j$ respectively. The terms $\varepsilon_{i j}$ and $\sigma_{i j}$ are the potential well depth and atomic diameter for the potential between atoms $i$ and $j$ (Figure 1.2). The parameter $r_{i j}$ is the distance between atoms $i$ and $j$.

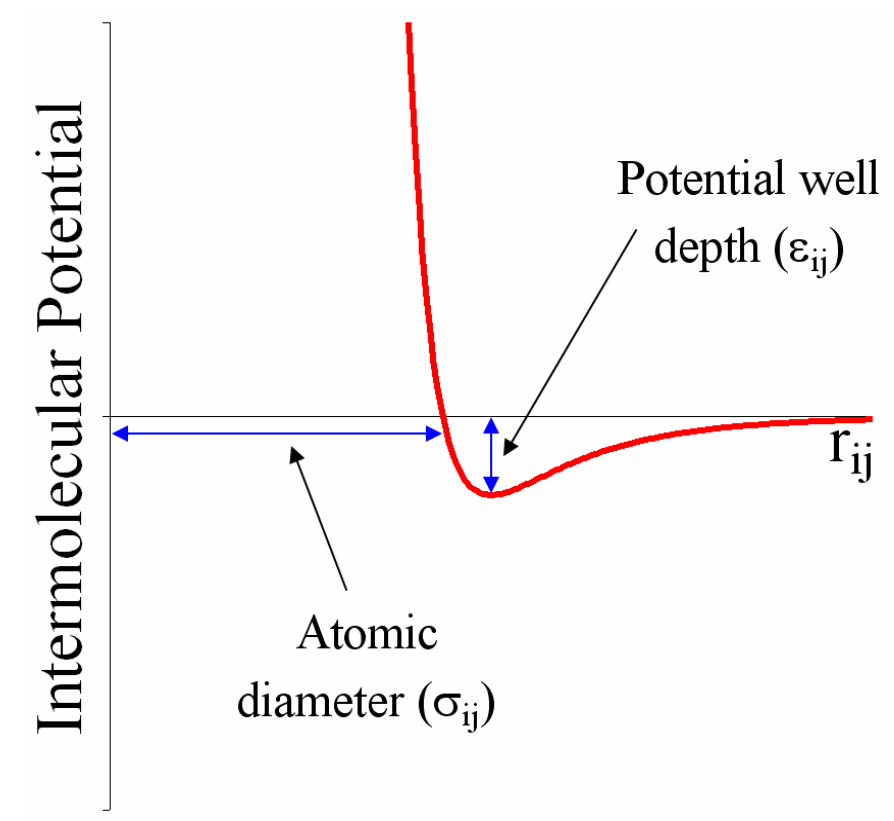

Figure 1.2: Intermolecular Potential

\subsection{SENSITIVITY STUdY}

A sensitivity study was performed to demonstrate how errors in the predicted energy of a rocket fuel can affect both propulsion level and vehicle level outputs. This study used the engine powerhead design code ROCETS [22] with a liquid oxygen, liquid hydrogen staged-combustion cycle rocket engine. The thermophysical properties of liquid hydrogen were altered to study the effects of changing these properties on vacuum $\mathrm{I}_{\mathrm{sp}}$. The purpose of this sensitivity study is twofold: (1) to see which of the thermophysical properties need to be measured most accurately, and (2) to see how errors in the predicted energy of a rocket fuel affect the vehicle level prediction of launch vehicle gross weight. 


\subsubsection{Overview of Conceptual Rocket engine Powerhead Design}

An overview of conceptual rocket engine powerhead design is first provided to describe a typical design code analysis, the engine components analyzed, and the thermophysical properties needed in each component analysis.

The main engine components designed are shown in the engine cycle diagram in Figure 1.3. This figure is taken from the REDTOP-2 cycle diagrams [23]. The main rocket engine components analyzed by powerhead design codes are the propellant tank, turbopump, main combustion chamber, and nozzle. Other key components are propellant feed lines and valves. Other main components for certain types of engine cycles are gas generators and preburners. The analysis of each of these components typically requires information about the thermodynamic and physical properties of the propellants moving through them. Figure 1.3 provides a listing of the thermophysical propellant properties needed for each component analysis.

Propellant density is used in sizing the fuel and oxidizer tanks. Density and kinematic viscosity are used in designing and analyzing turbopumps, propellant feed lines, and valves. Thermal conductivity is used in heat exchanger models to model the heat transfer from the main combustion chamber and nozzle into propellant flowing in the chamber and nozzle cooling jacket. Enthalpy and entropy are used in the combustor model (for the main combustion chamber and any other combustion devices such as gas generators or preburners). The combustor model is typically a one-dimensional chemical equilibrium model. If the fuel and oxidizer are assumed to exist as both reactants and products, then entropy data for the fuel and oxidizer is needed. If they are assumed to exist only as reactants, then only enthalpy data is needed.

With the thermophysical properties required for rocket engine powerhead design defined, sensitivity studies of changes in these properties on engine and vehicle level metrics can be performed. 


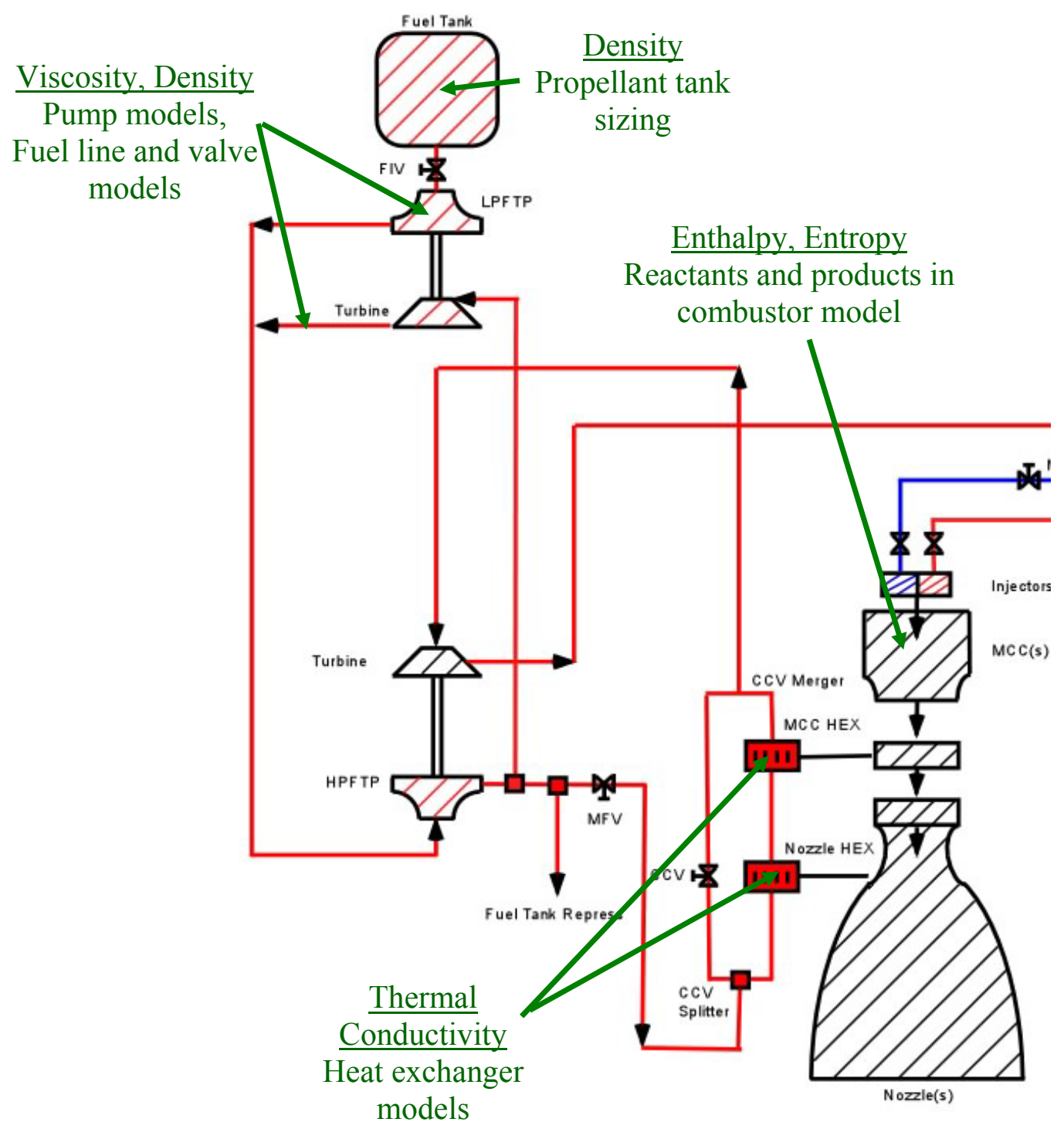

Figure 1.3: Expander Cycle Engine Diagram - Fuel Side

\subsubsection{ENGINE LEVEL SENSITIVITY}

The property prediction approach should ideally be tailored to the accurate prediction of the particular thermophysical properties that have the greatest influence on the engine level metrics. Those thermophysical properties that have less influence on engine level metrics can then be estimated using lower fidelity techniques to save time and resources. 
Figure 1.4 is a Pareto plot of engine vacuum $I_{s p}$ as a function of hydrogen fuel (both liquid and gaseous) enthalpy, entropy, density, kinematic viscosity, and thermal conductivity. A Pareto plot is used to determine the sensitivity of the response $\left(\mathrm{I}_{\mathrm{sp}}\right)$ to the various factors (enthalpy, entropy, density, kinematic viscosity, and thermal conductivity). The horizontal axis indicates the contribution of the variation of a particular factor to the total variation of the response. Each factor was changed by $20 \%$ to generate the data for this Pareto plot.

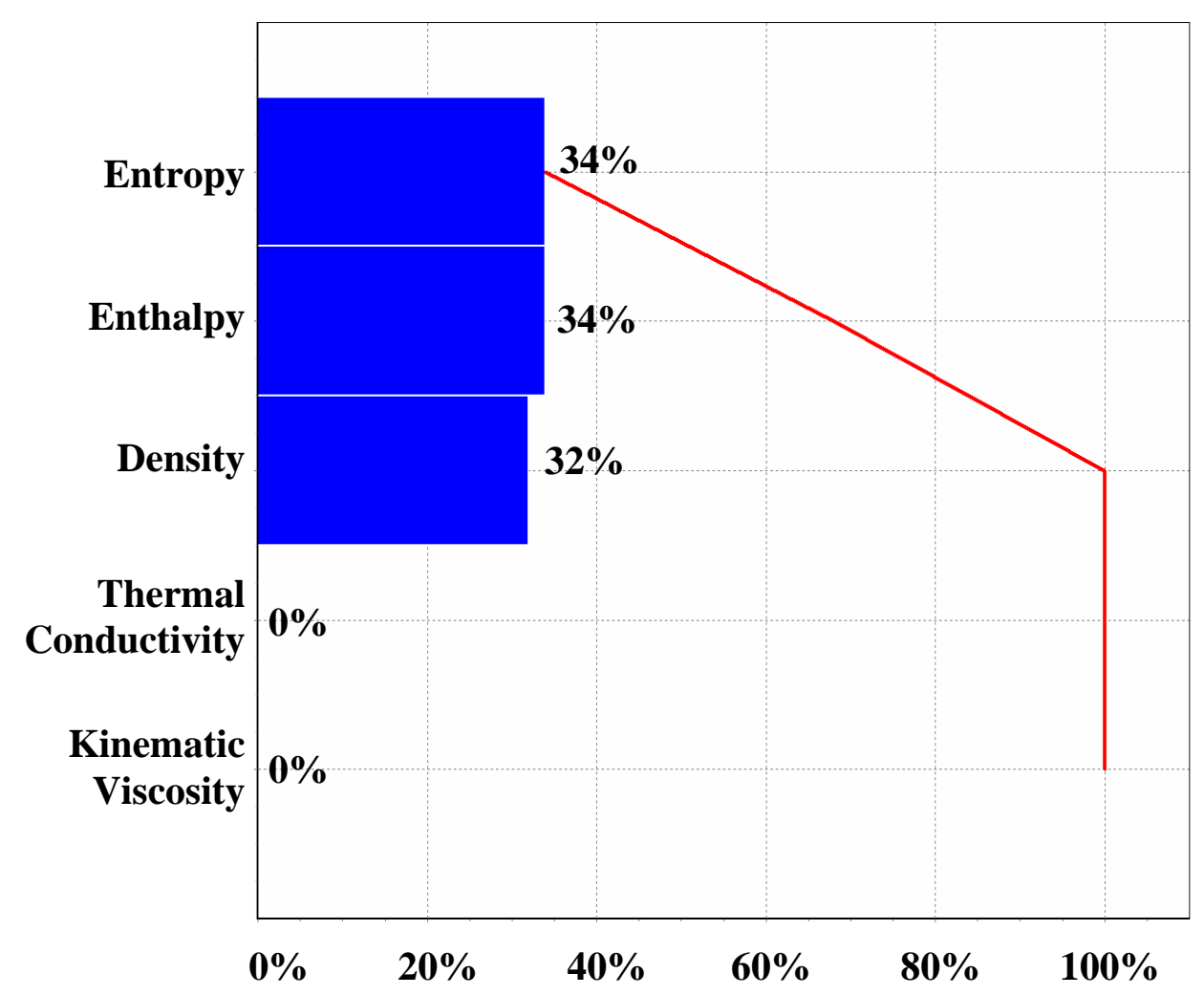

Figure 1.4: Pareto Plot of Vacuum $I_{\mathrm{sp}}$

The results shown in Figure 1.4 indicate that changes in enthalpy, entropy, and density have a much greater influence on $\mathrm{I}_{\mathrm{sp}}$ than do changes in kinematic viscosity and thermal conductivity. This indicates that enthalpy, entropy, and density need to be 
predicted with higher accuracy than do kinematic viscosity and thermal conductivity for conceptual liquid rocket engine design.

To better understand the influence of kinematic viscosity and thermal conductivity on $\mathrm{I}_{\mathrm{sp}}$, single variable sensitivity studies were performed. A multiplication factor was applied to both kinematic viscosity and thermal conductivity. This multiplication factor ranged from 0.2 to 5 . The results of this analysis are shown in Figure 1.5 for kinematic viscosity and Figure 1.6 for thermal conductivity. The results indicate that $\mathrm{I}_{\mathrm{sp}}$ changes by approximately 0.13 seconds $(0.029 \%)$ over the kinematic viscosity multiplier range and 0.14 seconds $(0.031 \%)$ over the thermal conductivity multiplier range. These results indicate that a lower fidelity technique can be used to estimate kinematic viscosity and thermal conductivity.

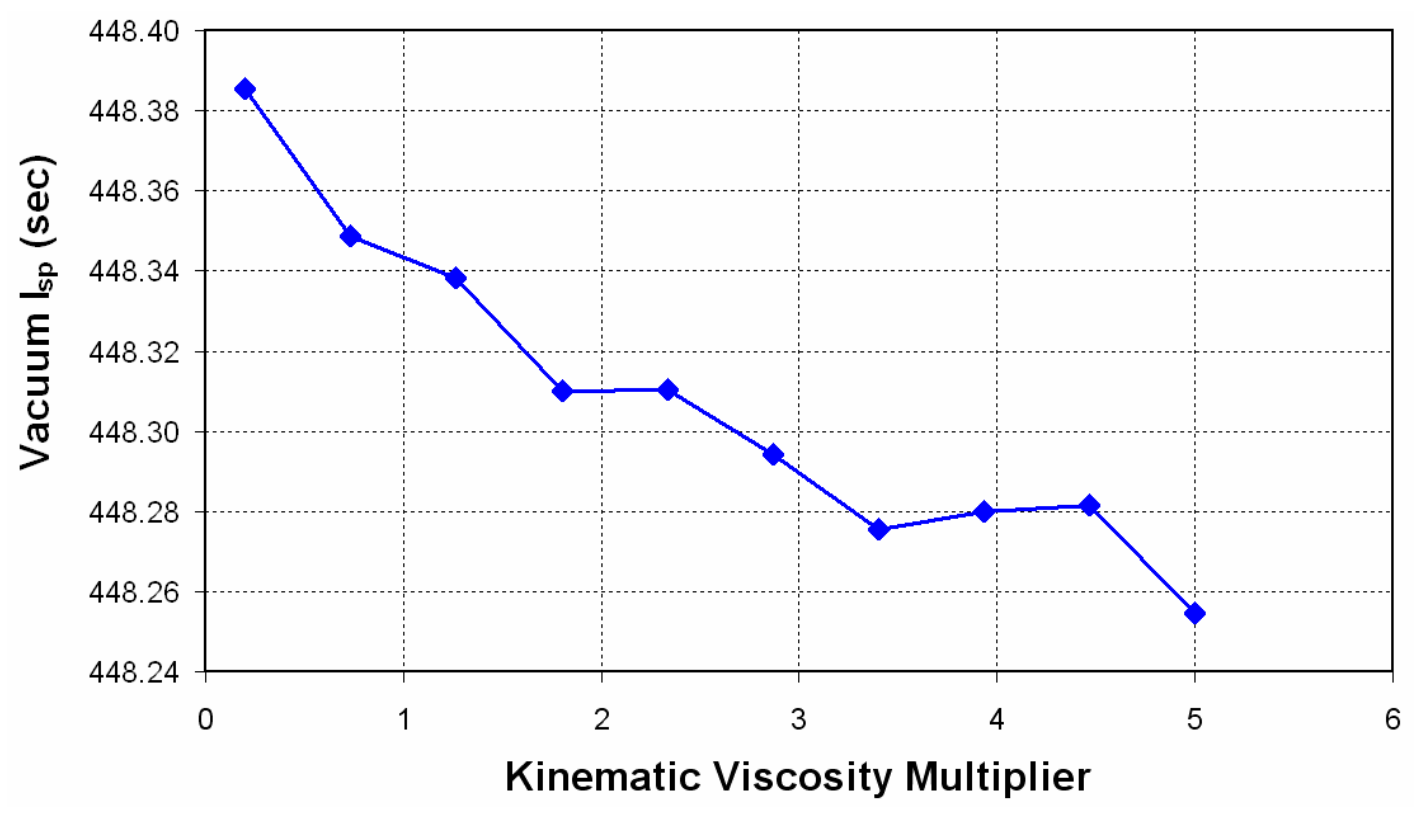

Figure 1.5: Vacuum $I_{s p}$ versus Kinematic Viscosity Multiplier

The results shown in Figure 1.5 and Figure 1.6 are not smooth due, most likely, to the internal tolerances of ROCETS. This is especially pronounced due to the insensitivity of $\mathrm{I}_{\mathrm{sp}}$ to changes in kinematic viscosity and thermal conductivity. 


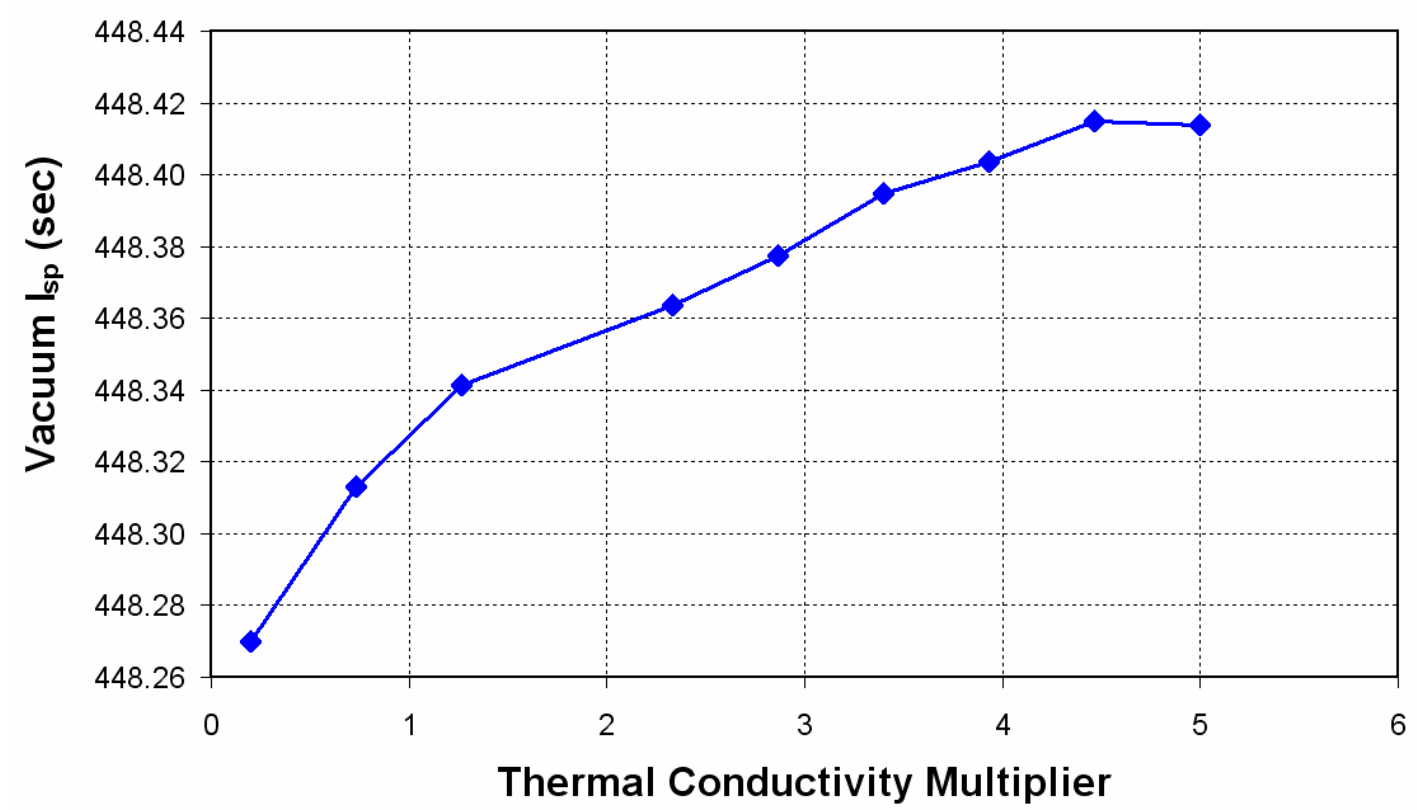

Figure 1.6: Vacuum $I_{\text {sp }}$ versus Thermal Conductivity

Table 1.2 provides the results of the engine level multi-variable sensitivity study. As can be seen from the table, changing the enthalpy, entropy, or density by $20 \%$ has a significant impact on vacuum $\mathrm{I}_{\mathrm{sp}}$.

Table 1.2: Thermophysical Property Sensitivity Study

\begin{tabular}{cccccc}
\hline $\begin{array}{c}\text { Enthalpy } \\
\text { Multiplier }\end{array}$ & $\begin{array}{c}\text { Entropy } \\
\text { Multiplier }\end{array}$ & $\begin{array}{c}\text { Density } \\
\text { Multiplier }\end{array}$ & $\begin{array}{c}\text { Kinematic } \\
\text { Viscosity } \\
\text { Multiplier }\end{array}$ & $\begin{array}{c}\text { Thermal } \\
\text { Conductivity } \\
\text { Multiplier }\end{array}$ & $\begin{array}{c}\text { Vacuum } \\
I_{s p}(\mathrm{sec})\end{array}$ \\
\hline 1.0 & 1.0 & 1.0 & 1.0 & 1.0 & 448.337 \\
1.2 & 1.0 & 1.0 & 1.0 & 1.0 & 441.538 \\
1.0 & 1.2 & 1.0 & 1.0 & 1.0 & 441.537 \\
1.0 & 1.0 & 1.2 & 1.0 & 1.0 & 452.436 \\
1.0 & 1.0 & 1.0 & 1.2 & 1.0 & 448.336 \\
1.0 & 1.0 & 1.0 & 1.0 & 1.2 & 448.342 \\
\hline
\end{tabular}

A $20 \%$ increase in enthalpy multiplier (the enthalpy at a given temperature is multiplied by 1.2) results in a 6.8 second decrease in vacuum $\mathrm{I}_{\mathrm{sp}}$. This 6.8 second decrease in $\mathrm{I}_{\mathrm{sp}}$ has a significant impact on vehicle level metrics such as a launch vehicle's gross weight. The fact that vacuum $\mathrm{I}_{\mathrm{sp}}$ reduces as enthalpy multiplier increases is due to 
the fact that the enthalpy of liquid hydrogen is negative. As a result, by increasing the enthalpy multiplier, we are actually reducing the energy available and thus the resulting $\mathrm{I}_{\mathrm{sp}}$.

\subsubsection{VEHICLE LEVEL SENSITIVITY}

In order to quantify the impact on launch vehicle gross weight of changing the vacuum $I_{\mathrm{sp}}$ by 6.8 seconds, a simple mass estimation analysis using the modified rocket equation was performed. The modified rocket equation relates the change in mass of a space vehicle in a given mission to the energy or $\Delta \mathrm{V}$ required to perform that mission. The modified rocket equation is shown in equation (3).

$$
\Delta V=g_{0} \bar{I}_{s p} \ln M R-\Delta V_{\text {Drag }}-\Delta V_{\text {Gravity }}-\Delta V_{T V C}
$$

In the above equation, $\Delta V$ is the overall change in velocity required to perform a particular mission, $\bar{I}_{s p}$ is the average specific impulse over the entire trajectory, $M R$ is the ratio of the vehicle's mass before firing the rocket engine to the vehicle's mass after firing the rocket engine, $\Delta V_{\text {Drag }}$ is the loss in velocity due to atmospheric drag, $\Delta V_{\text {Gravity }}$ is the loss in velocity to account for the energy required by the rocket's engine to counteract the force of gravity, and $\Delta V_{T V C}$ is the loss in velocity due to changing the direction of the velocity vector.

Using equation (3) along with assumptions regarding the amount of losses incurred in a launch trajectory from the surface of the Earth into a $120 \mathrm{~km}$ circular orbit, an analysis was performed to determine the change in mass of a launch vehicle as a result of a $20 \%$ increase in the enthalpy multiplier. An analysis was performed for both a single-stage and a two-stage launch vehicle. The mass results for the $20 \%$ decrease in enthalpy are shown in Table 1.3. We see that by increasing the enthalpy multiplier by 
$20 \%$, the gross weight of a single-stage launch vehicle increases by $74.2 \%$ and the gross weight of a two-stage launch vehicle increases by $8.4 \%$.

Table 1.3: Sensitivity Study - Launch Vehicle Mass Impact

\begin{tabular}{|c|c|c|c|c|}
\hline & \multicolumn{2}{|c|}{ Single-Stage Vehicles } & \multicolumn{2}{|c|}{ Two-Stage Vehicles } \\
\hline & Baseline & $\begin{array}{c}\text { Lower } \mathrm{LH}_{2} \\
\text { Enthalpy }\end{array}$ & Baseline & $\begin{array}{c}\text { Lower } \mathrm{LH}_{2} \\
\text { Enthalpy }\end{array}$ \\
\hline Vacuum $I_{s p}(\mathrm{sec})$ & 448.337 & 441.538 & 448.337 & 441.538 \\
\hline Non-Dimensional Gross Weight & 1.000 & 1.742 & 1.000 & 1.084 \\
\hline
\end{tabular}

The significant changes in gross vehicle weight caused by simply changing the enthalpy of the rocket engine fuel by $20 \%$ clearly show the need to accurately predict these thermophysical properties for conceptual rocket engine design.

\subsection{UNRESOLVED ISSUES AND GAPS IN KNOWLEDGE}

The COMPASS force field model does a good job of predicting energies and densities of compounds that are similar to those upon which it is parameterized (alkanes, alkenes, and certain ring compounds) [8,9]. However, this force field model has problems when attempting to model the more complex, high energy hydrocarbons that are often referred to as HEDM. HEDM propellants are more difficult to model using the COMPASS force field model because the strained bonds found in these HEDM propellants are not seen in the training set molecules used to create the original COMPASS force field.

In order to improve the predictive capabilities of the COMPASS model for HEDM propellants, alterations to the force field model need to be made. The coefficient values used in the COMPASS model need to be tailored to specific propellants in order to provide the most accurate prediction of the thermophysical properties of interest. 


\subsection{RESEARCH GOALS AND OBJECTIVES}

The overall goal of this project is to develop a thermophysical property calculation method which can be used to calculate the properties necessary for the conceptual design of rocket engine powerheads. This calculation method must be repeatable and implementable in a reasonable amount of time for use in conceptual design. Listed below are the specific objectives required to achieve this goal:

Objective 1: The process of predicting the thermophysical properties of potential new liquid rocket propellants should primarily be analytical / numerical. It should require little or no new experimental work.

This objective is necessary in order to allow this method to be incorporated into a conceptual design environment in which a limited amount of time and resources are available. As a result, the prediction of the necessary thermophysical properties must be done relatively quickly without the need for expensive and time-consuming experimental work.

Objective 2: Predict the density of HEDM molecules to within $10 \%$ of the experimentally measured value. Predict the total enthalpy and total entropy to within 5\% of the experimentally measured value. Predict the specific heat at constant pressure to within $10 \%$ of the experimentally measured value. Do this by improving the predictive capability of the COMPASS force field model.

The COMPASS force field model is a general force field model that can be used on a wide range of hydrocarbon molecules to predict condensed-phase thermophysical properties [8,9], and as a result, it is a good initial model for predicting the thermophysical properties of hydrocarbon HEDM molecules. However, due to the fact 
that the COMPASS model was created with the goal of producing fairly accurate predictions of molecular properties for alkanes, alkenes, and alkynes [8], it is not specifically designed to model hydrocarbon HEDM molecules. The research work described in this thesis addresses the COMPASS model predictive inaccuracy for HEDM molecules by tailoring the coefficients of the COMPASS force field model to HEDMtype molecules.

\section{Objective 3: Predict the kinematic viscosity and thermal conductivity of HEDM molecules so that the predicted values fall within a multiplication range of $40 \%$ to $250 \%$ of the experimental values.}

As was shown by the sensitivity study analysis in section $1.2, \mathrm{I}_{\mathrm{sp}}$ is less sensitive to changes in kinematic viscosity and thermal conductivity than it is to changes in enthalpy, entropy, and density. A multiplication range of $20 \%$ to $500 \%$ was applied to the values of kinematic viscosity and thermal conductivity at a given temperature and pressure. The sensitivity study results indicated that $I_{\text {sp }}$ changes by approximately 0.13 seconds over the kinematic viscosity multiplier range and 0.14 seconds over the thermal conductivity multiplier range. Due to the likelihood that changes in kinematic viscosity and thermal conductivity could have a larger impact on $\mathrm{I}_{\mathrm{sp}}$ for different engine cycles and for different powerhead design codes, the multiplication range decreased by a factor of two from both ends $(40 \%$ instead of $20 \%$ on the lower end and $250 \%$ instead of $500 \%$ on the upper end).

\subsection{DisSERTATION OUTLINE}

The rest of this dissertation is broken down into five chapters. Chapter 2 provides a background of propellant characterization techniques. Chapter 3 presents the methodology implemented in this thesis research to predict the thermophysical properties 
necessary for conceptual rocket engine powerhead design codes. Chapter 4 provides the results from implementing this methodology on two HEDM propellants, quadricyclane and DMAZ, and their corresponding model compounds, norbornane and ethyl azide. Chapter 5 is a conceptual vehicle design case study utilizing the thermophysical property calculations from Chapter 4. The vehicle designed is the Lunar Surface Access Module (LSAM) from the NASA Exploration Systems Architecture Study (ESAS). Chapter 6 provides conclusions and recommendations for future work. 


\section{CHAPTER 2}

\section{BACKGROUND}

An overview of the analytical and numerical methods available to predict the thermophysical properties used by conceptual rocket engine powerhead design codes is provided in this chapter. The relative advantages and disadvantages of each method are discussed.

\subsection{Evolution of Modeling Techniques to Predict Properties}

A review of the methods used to predict the thermophysical properties of molecules has shown that there are three main techniques that can be used. The first is a technique of fitting equations of state to experimental data to relate thermodynamic properties to one another. Examples of this method range from the simple ideal gas equation of state (equation (4)), to the more complex van der Waals (equation (5)) and Benedict-Webb-Rubin (equation (6)) equations of state [24, pp.47-49].

$$
\begin{gathered}
P=\rho R T \\
\left(P+\frac{a}{V_{m}^{2}}\right)\left(V_{m}-b\right)=R T \\
P=\rho R T+\rho^{2}\left(R T B_{0}-A_{0}-C_{0} / T^{2}\right)+\rho^{3}(R T b-a)+\rho^{6}(a \alpha)+\frac{\rho^{3} c}{T^{2}}\left(1+\rho^{2} \gamma\right) e^{-\rho^{2} \gamma}
\end{gathered}
$$

In the above equations, $P$ is the pressure of the system, $\rho$ is the density, $R$ is the gas constant for a particular substance, $T$ is the temperature, and $V_{m}$ is the molar volume. The parameters $a$ and $b$ in equation (5) and $a, b, c, A_{0}, B_{0}, C_{0}, \gamma$, and $\alpha$ in equation (6) are coefficients that are specific to a particular substance. 
This method of making use of relatively simple equations that approximate the properties of real substances has been used extensively for centuries. In 1662, Robert Boyle developed what is now known as Boyle's Law. Boyle's law states that the product of volume and pressure is a constant for an ideal gas when temperature is held constant [25]. Over a century later in 1787 , Jacques Charles developed what was later named Charles's Law. Charles's law states that the ratio of volume to temperature of an ideal gas is constant when pressure is held constant [25]. These two laws together form the basis for the ideal gas equation of state shown in equation (4).

Equations of state are typically valid for a single phase (gas, liquid, or solid) and make use of several empirical constants. These equations relate a predicted physical property to other known properties. Figure 2.1 [26] is an example of this type of relationship. Figure 2.1 is a graph of the critical temperature versus normal boiling point for 535 chemicals. The critical temperature of a substance is the maximum temperature at which liquid and vapor phases of that substance can coexist in equilibrium [27, p.86].

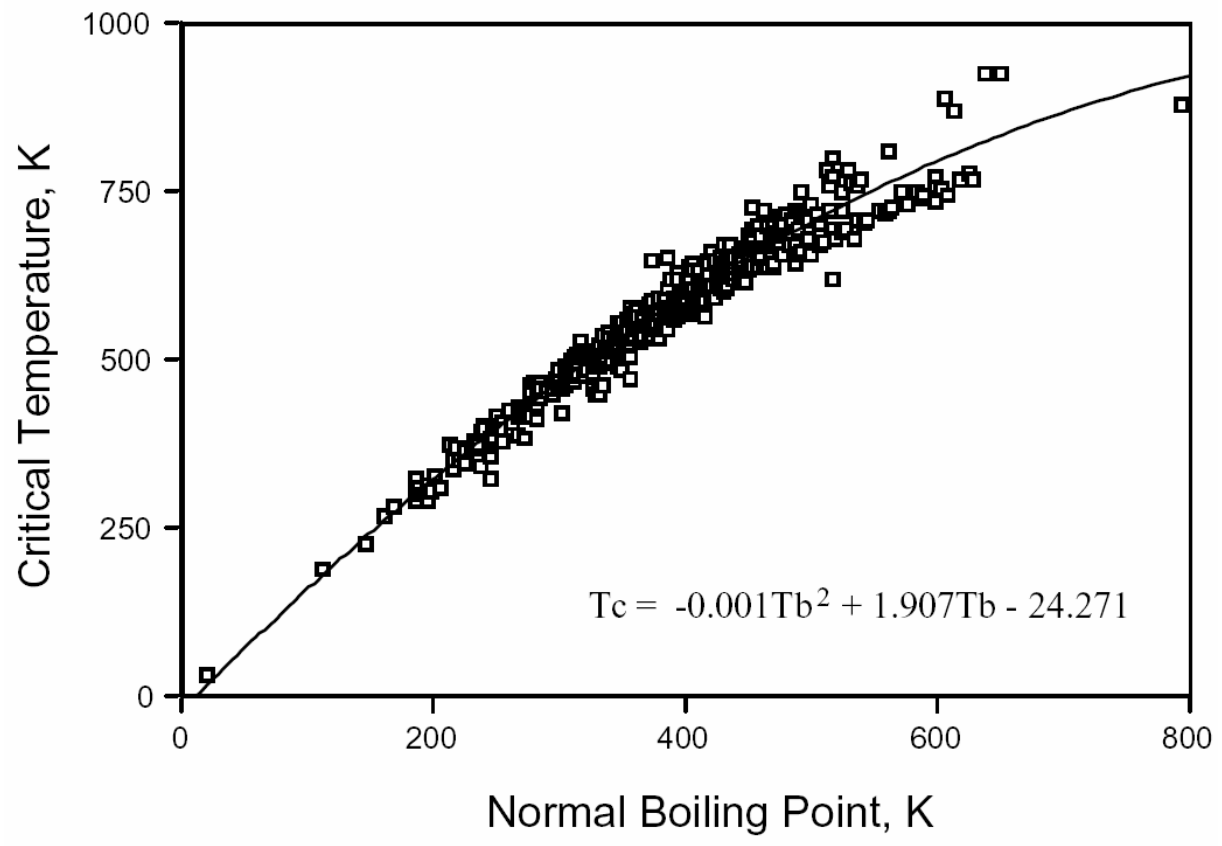

Figure 2.1: Critical Temperature-Boiling Point Relationship [26] 
A general trend can be seen from the graph that can be captured with a quadratic equation relating the critical temperature to the boiling point temperature. The average absolute deviation of this regression, for these 535 chemicals, is less than $4 \%$. As can be seen from the graph, however, the deviation tends to increase as the normal boiling point increases. Limits must be placed on the validity of these types of equations as they are typically only valid over some range of temperature and/or pressure. As a result, when used outside their range of validity, these types of equations provide inaccurate results for new molecules such as the HEDM propellants mentioned previously.

A second technique for predicting the thermophysical properties of substances makes use of the observation that a substance's physical properties depend on that substance's particular molecular structure [26,28, p.24]. Benson and Buss [29] in 1958 showed that it was possible to make a system of "additivity rules" to determine certain thermodynamic and physical properties of substances based upon their atom, bond, and group makeup. Individual contributions from atoms, bonds, and groups to the estimated values of thermophysical properties can be calculated by regressing empirical thermophysical data for known substances. Table 2.1 [28, p.25] and Table 2.2 [28, p.273] show the results of such a regression for the bond and ring contributions to specific heat at constant pressure $\left(C_{p}^{o}\right)$, total entropy $\left(S^{o}\right)$, and the heat of formation $\left(\Delta h_{f}^{o}\right)$ for ideal gases at $25^{\circ} \mathrm{C}$ and $1 \mathrm{~atm}$.

Table 2.1: Bond Contributions for the Estimation of $C_{p}{ }^{o}, S^{o}$, and $\Delta h_{f}^{o}[28$, p.25]

\begin{tabular}{cccc}
\hline Bond & $C_{p}{ }^{o}(\mathrm{cal} / \mathrm{mole}-\mathrm{K})$ & $S^{o}(\mathrm{cal} / \mathrm{mole}-\mathrm{K})$ & $\Delta h_{f}^{o}(\mathrm{kcal} / \mathrm{mole})$ \\
\hline $\mathrm{C}-\mathrm{H}$ & 1.74 & 12.90 & -3.83 \\
$\mathrm{C}-\mathrm{C}$ & 1.98 & -16.40 & 2.73 \\
$\mathrm{C}-\mathrm{F}$ & 3.34 & 16.90 & -52.50 \\
$\mathrm{C}-\mathrm{Cl}$ & 4.64 & 19.70 & -7.40 \\
$\mathrm{C}-\mathrm{O}$ & 2.70 & -4.00 & -12.00 \\
\hline
\end{tabular}


Table 2.2: Ring Corrections for the Estimation of $C_{p}^{o}, S^{o}$, and $\Delta h_{f}^{o}[28$, p.273]

\begin{tabular}{cccc}
\hline Ring & $C_{p}{ }^{o}(\mathrm{cal} / \mathrm{mole}-\mathrm{K})$ & $S^{o}(\mathrm{cal} / \mathrm{mole}-\mathrm{K})$ & $\Delta h_{f}^{o}(\mathrm{kcal} / \mathrm{mole})$ \\
\hline Cyclopropane & -3.05 & 32.10 & 27.60 \\
Cyclobutane & -4.61 & 29.80 & 26.20 \\
Cyclopentane & -6.50 & 27.30 & 6.30 \\
\hline
\end{tabular}

If one wished to use these additivity rules to calculate $C_{p}{ }^{o}$ of $\mathrm{CHCl}_{3}$ (Figure 2.2) at $25^{\circ} \mathrm{C}$ and $1 \mathrm{~atm}$, one would calculate it in the following way:

$C_{p}^{o}\left(\mathrm{CHCl}_{3}\right)=C_{p}^{o}(\mathrm{C}-\mathrm{H})+3 * C_{p}^{o}(\mathrm{C}-\mathrm{Cl})=1.74+3 * 4.64=15.66 \mathrm{cal} / \mathrm{mole}-\mathrm{K}$

The calculated value of $C_{p}{ }^{o}\left(\mathrm{CHCl}_{3}\right)$ of $15.66 \mathrm{cal} / \mathrm{mole}-\mathrm{K}$ is only $0.25 \%$ below the empirically measured value of $15.70 \mathrm{cal} / \mathrm{mole}-\mathrm{K}[28, \mathrm{p} .25]$. This high level of accuracy is common for these types of small molecules that do not contain strained-rings and are not heavily branched [28, p.26].

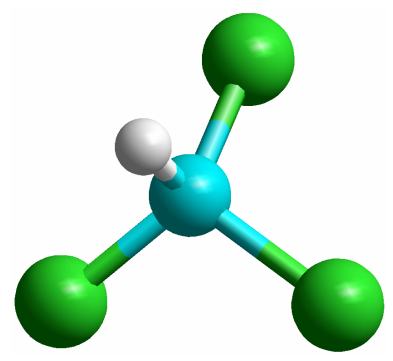

Figure 2.2: Ball and Cylinder Rendering of Chloroform $\left(\mathrm{CHCl}_{3}\right)$

While this technique of using additivity rules works well for relatively simple molecules, it does not fare as well when used with more complex molecules such as HEDM molecules. This is because the values for the various bond (Table 2.1) and ring (Table 2.2) contributions are obtained from a regression of thermodynamic and physical data of relatively simple molecules. Using these same additivity rules on a HEDM 
molecule, quadricyclane (Figure 2.3), we can see the inability of these additivity rules to accurately measure thermodynamic properties.

$$
\begin{aligned}
\Delta h_{f}^{o}(\text { Quad })= & 8 * \Delta h_{f}^{o}(C-H)+10 * \Delta h_{f}^{o}(C-C)+ \\
& 2 * \Delta h_{f}^{o}(\text { Ring }- \text { cyclopropane })+\Delta h_{f}^{o}(\text { Ring }- \text { cyclobutane }) \\
= & 8 *-3.83+10 * 2.73+2 * 27.60+26.20=78.06 \mathrm{kcal} / \text { mole } \\
C_{p}^{298 K}(\text { Quad })= & 8 * C_{p}^{298 K}(C-H)+10 * C_{p}^{298 K}(C-C)+ \\
& 2 * C_{p}^{298 K}(\text { Ring }- \text { cyclopropane })+C_{p}^{298 K}(\text { Ring }- \text { cyclobutane }) \\
= & 8 * 1.74+10 * 1.98+2 *-3.05-4.61=23.01 \text { cal / mole }-K
\end{aligned}
$$

The calculated value of $\Delta h_{f}^{o}($ Quad) of $78.06 \mathrm{kcal} / \mathrm{mole}$ is $8.12 \%$ above the experimentally measured value of $72.2 \mathrm{kcal} / \mathrm{mole}[5,30]$. The calculated value of $C_{p}{ }^{298 K}$ (Quad) of $23.01 \mathrm{cal} / \mathrm{mole}-\mathrm{K}$ is $37.17 \%$ below the experimentally measured value of $36.62 \mathrm{cal} / \mathrm{mole}-\mathrm{K}[1]$. These deviations are higher than desired for $\Delta h_{f}^{o}$ and $C_{p}{ }^{298 K}$ for use in conceptual rocket engine powerhead design. Another disadvantage of using additivity rules is the limited range of temperatures and pressures in which the rules may be valid. The high temperatures and pressures found in a liquid rocket engine are outside the range of applicability of most additivity rules. When predictions are made outside a particular additivity rule's range of applicability, predictive errors increase.

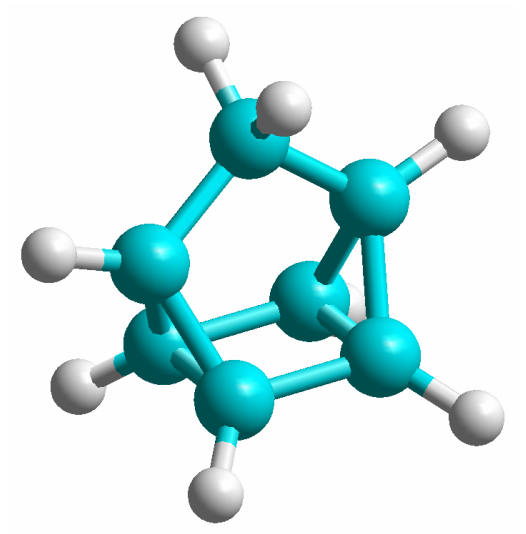

Figure 2.3: Ball and Cylinder Rendering of Quadricyclane $\left(\mathrm{C}_{7} \mathrm{H}_{8}\right)$ 
With the advent of computer simulations, a third method for predicting the thermophysical properties of substances was developed. This third method is molecular dynamics simulation [31, pp.1-3]. This is a method for modeling the behavior of solids, liquids, or gases by modeling the interactions of the individual particles that make up the solid, liquid, or gas. In molecular dynamics, the classical many-body problem is typically solved with Newtonian mechanics governing the movement of particles [10, pp.1-2]. For a polyatomic molecule, the molecule can be either rigid or flexible. A flexible analysis allows for internal motion, which results in changes in internal energy, but typically requires a smaller time step to capture high frequency internal vibrations.

A potential force field function is prescribed to the particles to model their influence on one another. This force field function is different for different molecules. With the correct force field model, the dynamics and interactions of the particles with one another can be accurately modeled. By modeling these interactions accurately, one can predict all the necessary thermodynamic and physical properties of the fluid.

\subsection{Chronology OF MOLECUlar DyNAMics THEORY AND RESEARCH}

A variety of potential force field models exist for use in molecular dynamics. The simplest, oldest, and most widely studied potential function is the Lennard-Jones potential shown in equation (10):

$$
u^{L J}\left(r_{i j}\right)=4 \varepsilon_{i j}\left[\left(\frac{\sigma_{i j}}{r_{i j}}\right)^{12}-\left(\frac{\sigma_{i j}}{r_{i j}}\right)^{6}\right]
$$

In the above equation, $u^{L J}\left(r_{i j}\right)$ is the energy potential for a pair of particles $i$ and $j$; $r_{i j}$ is the distance between particles $i$ and $j ; \varepsilon_{i j}$ is the Lennard-Jones potential well depth, which describes the strength of the interaction; and $\sigma_{i j}$ is the Lennard-Jones atomic diameter [31, p.32]. The Lennard-Jones potential was developed by Sir John Lennard- 
Jones in 1931 and was originally used in molecular dynamics simulations in the 1960's for the modeling of liquid argon [10, pp.11-12]. This potential has two terms; the first provides a strong repulsion at close range and the second provides a weak attraction at longer ranges (Figure 2.4).

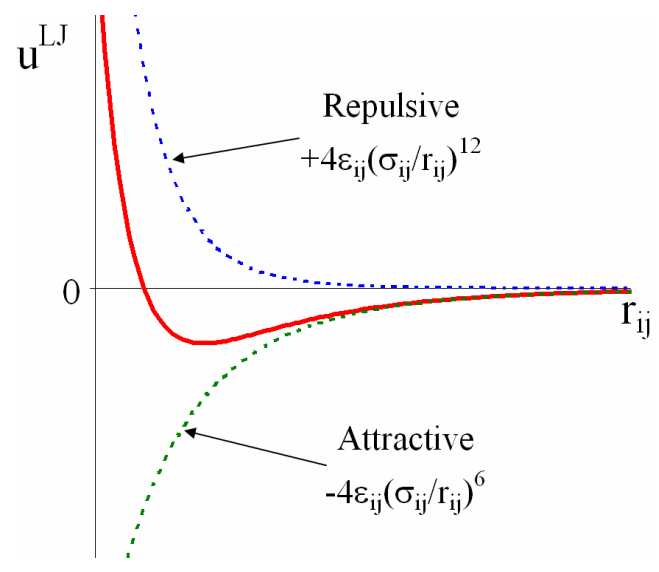

Figure 2.4: Lennard-Jones Intermolecular Potential

These terms represent respectively the nonbonded overlap of electron clouds of two different atoms and the van der Waals interaction due to dispersion forces caused when the electrons are not uniformly distributed around an atom or molecule [32, pp.66$67,33]$.

Although the Lennard-Jones potential is only accurate for very simple molecules such as argon, a great deal can be learned about the qualitative behavior of simple fluids with regards to phase equilibria, melting, vaporization, particles in small clusters, and surface and transport properties [34] through its study.

As computational power continued to increase, more accurate (and more computationally intensive) molecular potentials were developed in order to model more complex molecules. In the late 1970's and early 1980's, force field models such as MM2 [11], AMBER [12,13,14,15], and CHARMM [16,17] were developed for this purpose. 
These force field models all attempt to model not only the intermolecular forces between molecules, but also the molecular structure itself and the intramolecular forces that produce that structure. These models differ in the way they handle cross terms and the anharmonic nature of the vibrating bonds [8]. A cross term in a potential function is a term that includes functions of two or more internal coordinates (bond length, bond angle, and dihedral angle) multiplied together. It is typically incorporated into a potential function to attempt to capture the dependence of the internal coordinates upon one another [8]. It has been shown that the inclusion of the appropriate cross terms in a potential function is important in accurately predicting thermophysical properties [21].

In the early 1990's, improved force field models were developed to predict the thermophysical properties of condensed-phase materials. This is important for the prediction of liquid rocket propellant properties as these propellants exist typically in either liquid or supercritical form while in the various engine components such as the pump, turbine, feed lines, nozzle cooling jacket, and injector. New versions of the AMBER [35] and CHARMM [36] force field models both aim at improving the prediction of condensed-phase properties, as does the OPLS/CHARMM force field model [37].

The CFF93 force field model $[18,19]$ was developed in 1993 to achieve a very accurate prediction of molecular properties with a broad coverage of different types of molecules. The CFF93 model parameters were derived using quantum mechanical data for the intramolecular terms and molecular crystal data for the intermolecular terms. An error with this method became apparent when simulations run at elevated temperatures and liquid densities found that the calculated physical parameters did not match the experimental data.

As stated previously, molecular crystal data was used to determine the values for the intermolecular parameters $\left(q_{i}, q_{j}, \sigma_{i j}\right.$, and $\varepsilon_{i j}$ in the last two terms of equation (2)). This molecular crystal data was measured at non-zero temperatures. However, the 
calculations performed to match the molecular crystal data were run at $0 \mathrm{~K}[8]$. As a result, the calculated data will not be equal to the measured data, because the calculations were performed at one temperature while the measurements were taken at a different temperature. However, the values of the intermolecular parameters for the CFF93 model were set (using a least-squares fit) to force the calculated data equal to the measured molecular crystal data.

In an attempt to resolve the problem of experimental data and corresponding calculations at two different temperatures, the COMPASS force field model [8,9] was developed in 1998. Like CFF93, the COMPASS force field model parameters for the intramolecular terms were derived from curve fits of quantum mechanical data. The major improvement of the COMPASS force field model over the CFF93 force field model is the way in which the intermolecular parameters are determined. The intermolecular parameters for the COMPASS model are calculated by running molecular dynamics simulations at finite temperatures and fitting the simulated thermophysical results to experimental data by adjusting the intermolecular parameters $\left(\sigma_{i j}\right.$ and $\left.\varepsilon_{i j}\right)$. In the COMPASS model development, unlike the development of the intermolecular parameter values for the CFF93 model, both the simulation and the corresponding experimental data are at the same temperature. By determining the intermolecular parameters in this fashion, good agreement was reached between the calculated thermophysical properties and experimental data $[8,9]$.

Using the baseline COMPASS force field model, molecular dynamics simulations of liquid benzene and liquid DMAZ were performed as part of this thesis research work using the Large-scale Atomic/Molecular Massively Parallel Simulator (LAMMPS) molecular dynamics software [38]. Benzene was chosen because it is an example of a compound that the baseline COMPASS model does a good job of predicting thermophysical properties. This is due to the fact that benzene is one of the training set compounds used in determining the appropriate values for the COMPASS model 
parameters. DMAZ was chosen because it is an example of a HEDM compound that the baseline COMPASS model does a poor job of predicting thermophysical properties due to its high energy strained-bond structure. The structure of DMAZ is significantly different from that of the COMPASS model training set compounds.

A ball and cylinder rendering of a DMAZ molecule is shown in Figure 2.5. Figure 2.6 - Figure 2.9 show the density and enthalpy change results from these molecular dynamics simulations. The predictions of density and enthalpy change of liquid benzene match experimental data very well due to the fact that benzene is one of the COMPASS model training set compounds. However, the COMPASS model does a poor job of matching experimental data for liquid DMAZ, because the molecular structure of DMAZ is different from the alkanes, alkenes, and alkynes upon which the COMPASS model coefficients are based. All results are for a pressure of $1 \mathrm{~atm}$.

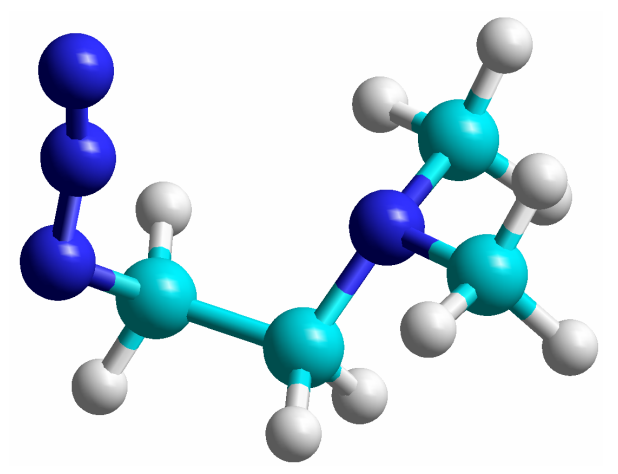

Figure 2.5: Ball and Cylinder Rendering of DMAZ $\left(\mathrm{C}_{4} \mathrm{H}_{10} \mathrm{~N}_{4}\right)$

The average root mean square (RMS) deviation over the temperature range simulated for the density of liquid benzene is $0.4 \%$. The average molecular dynamics simulated value for $C_{p}$ over the temperature range simulated is $28.4 \mathrm{cal} / \mathrm{mol}-\mathrm{K}$ which is $2.2 \%$ below the experimentally measured value of $29.0 \mathrm{cal} / \mathrm{mol}-\mathrm{K}$ [39]. 


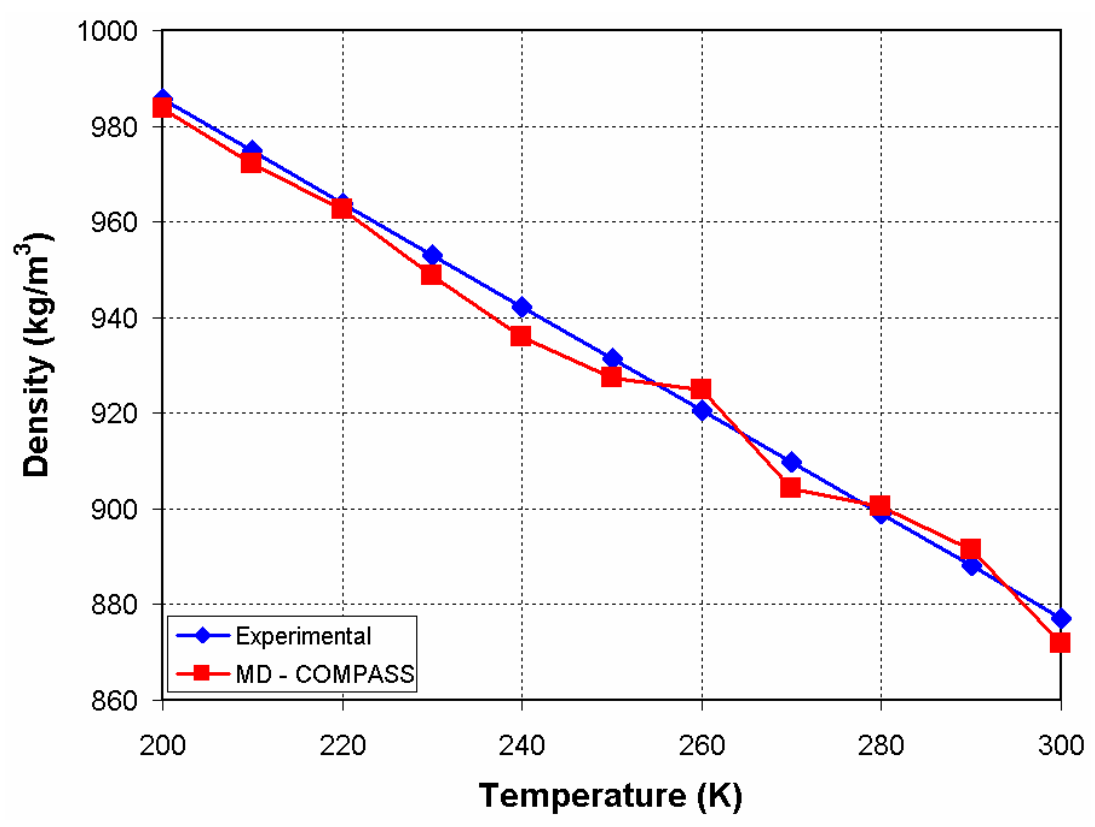

Figure 2.6: Benzene Experimental and MD Density vs. Temperature

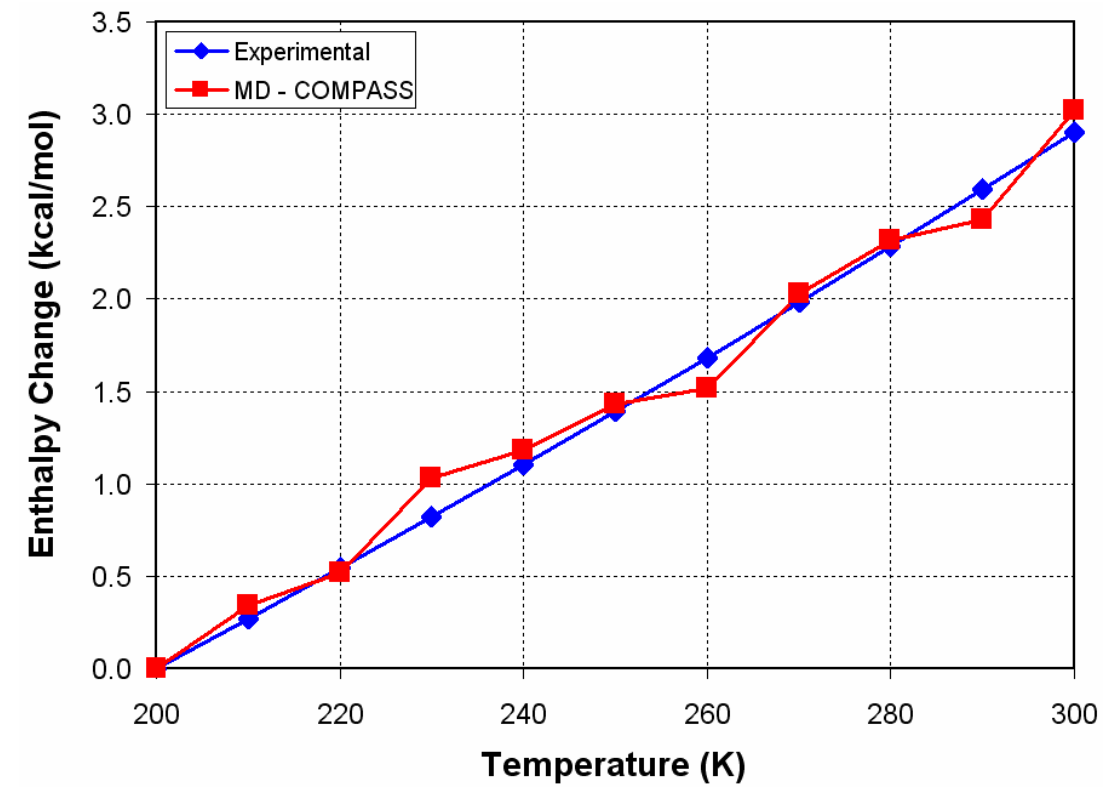

Figure 2.7: Benzene Experimental and MD Enthalpy Change vs. Temperature 
The average RMS deviation over the temperature range simulated for the density of liquid DMAZ is $15.8 \%$. The average molecular dynamics simulated value for $C_{p}$ over the temperature range simulated is $69.0 \mathrm{cal} / \mathrm{mol}-\mathrm{K}$ which is $36.9 \%$ above the experimentally measured value of $50.4 \mathrm{cal} / \mathrm{mol}-\mathrm{K}$.

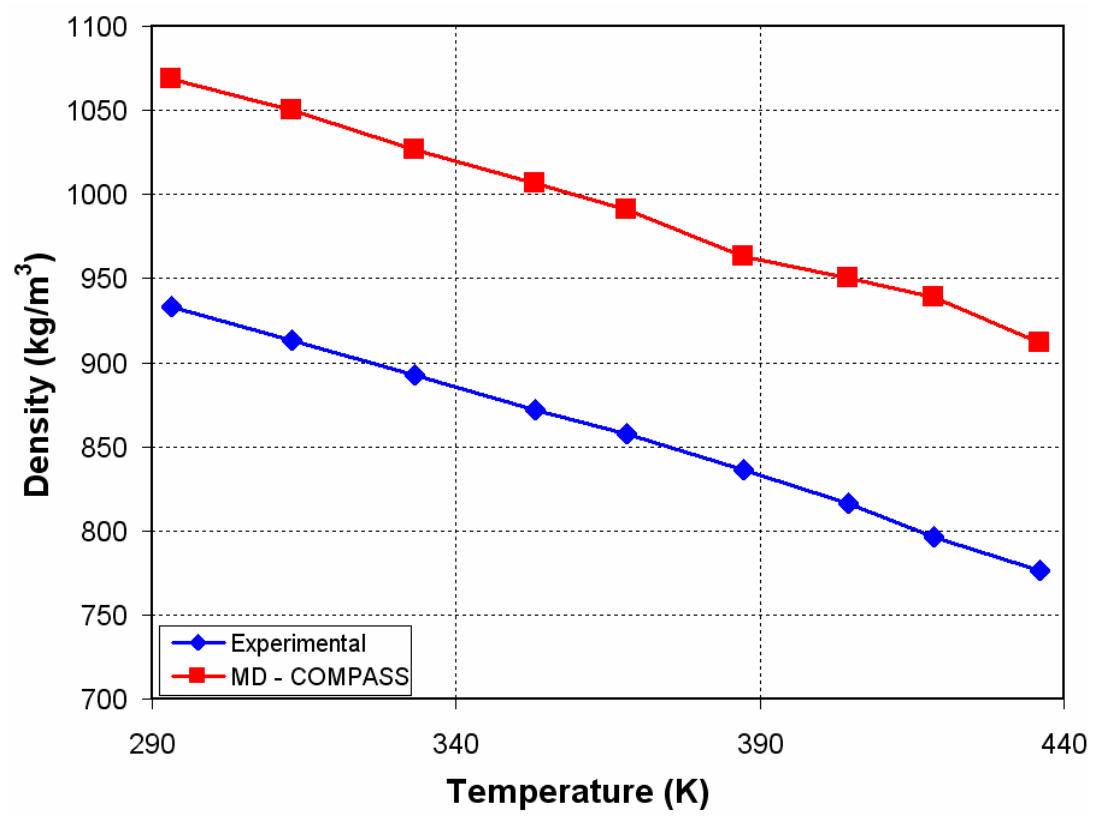

Figure 2.8: DMAZ Experimental and MD Density vs. Temperature

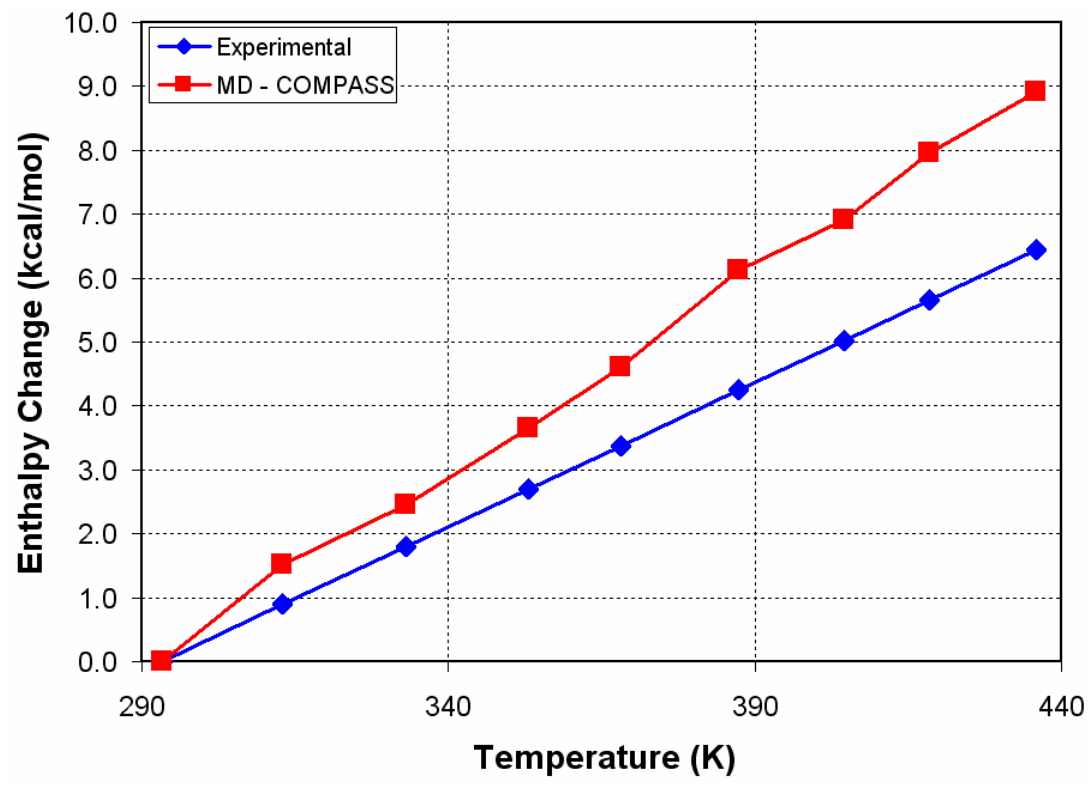

Figure 2.9: DMAZ Experimental and MD Enthalpy Change vs. Temperature 
These poor results for DMAZ show a clear need to improve the COMPASS force field model in order to more closely match experimental data. Property calculations and molecular dynamics modeling techniques used to produce these results are discussed in detail in the following chapter. 


\section{CHAPTER 3}

\section{THERMOPHYSICAL PROPERTY CALCULATION METHOD}

The method developed to calculate the necessary thermophysical properties for HEDM propellants consists of three main steps. First is the use of quantum mechanical energy calculations to determine the kinetic energy and intramolecular potential energy of the compound of interest as a function of temperature. Second is the application of molecular dynamics to determine the density, enthalpy change, and entropy change of the compound of interest at a range of temperatures and pressures. Third is the use of additivity methods to determine the kinematic viscosity and thermal conductivity of the compound of interest over the same temperature and pressure ranges. Together these three steps enable the accurate prediction of all the necessary thermophysical properties of rocket fuels for conceptual rocket engine powerhead design. Figure 3.1 - Figure 3.4 detail these steps in flow chart form, with Figure 3.1 providing an overview of the entire property calculation method.

\subsection{Property Calculation OVerview}

The property calculation method starts with an initial molecular configuration of the HEDM compound and corresponding model compound. These molecular configurations are provided either from published data or from quantum mechanical energy minimization. A method developed by Lagache et al. [40] and Cadena et al. $[41,42]$ is used to determine the enthalpy and specific heat of the liquid HEDM and model compounds as a function of temperature and pressure. This method breaks down the calculation of enthalpy (and specific heat) into ideal gas and residual components. The ideal gas component is determined through the use of quantum mechanically 
calculated normal mode vibrational frequencies (Figure 3.2). The residual component is determined through molecular dynamics simulation.

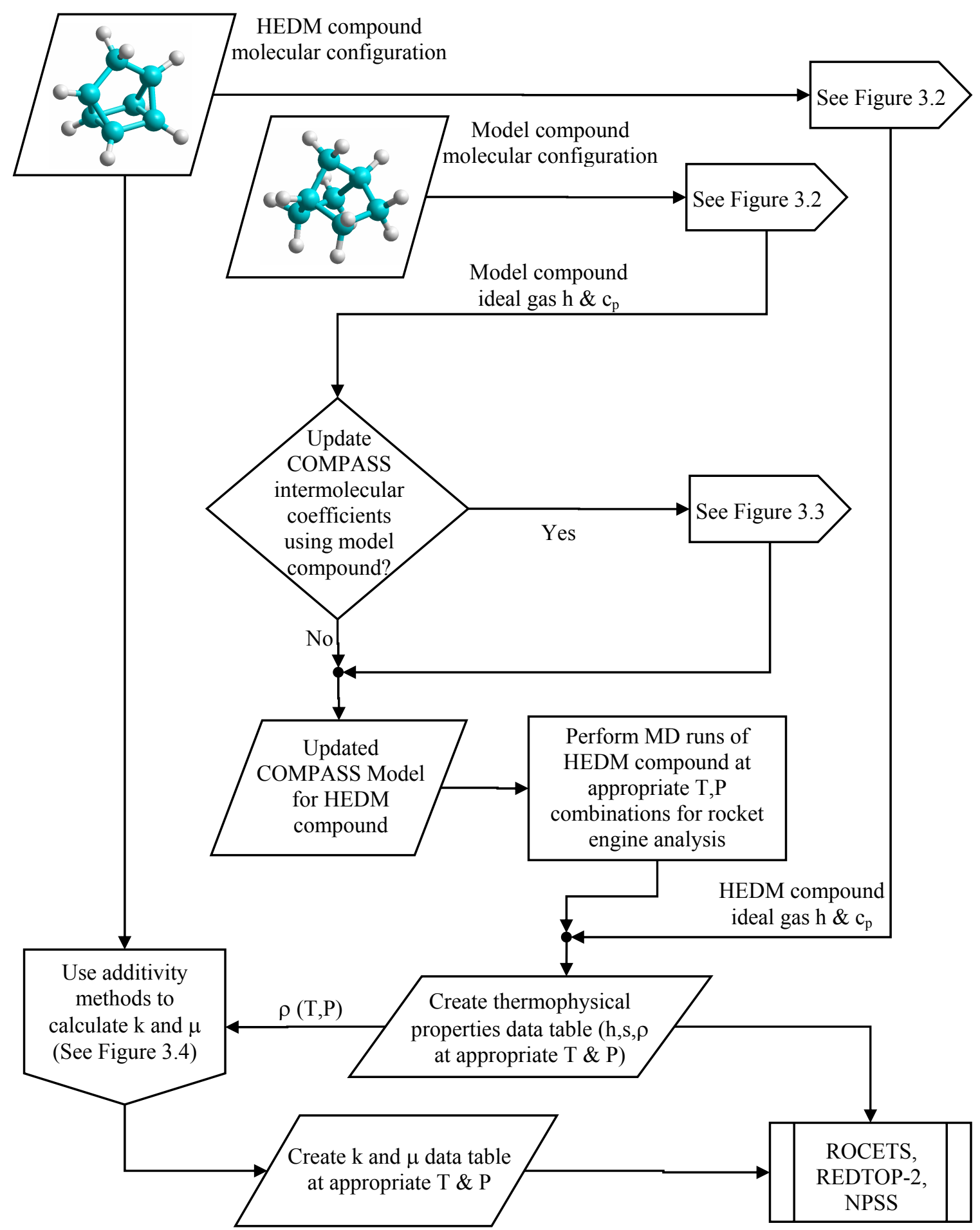

Figure 3.1: Thermophysical Property Calculation Method Overview 


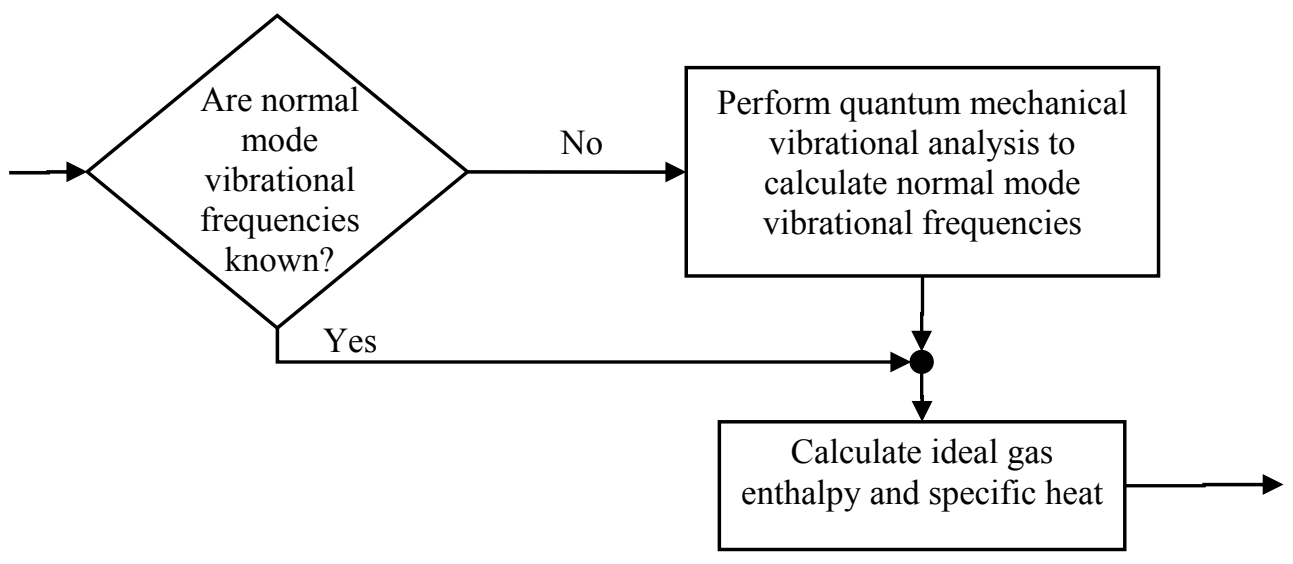

Figure 3.2: Part I: Quantum Mechanics

The following equations describe how the specific heat at constant pressure $\left(C_{p}\right)$ is broken down into ideal gas and residual components:

$$
\begin{gathered}
C_{P}(T, P)=C_{P}^{i g}(T)-R+C_{P}^{\text {res }}(T, P)=C_{V}^{i g}(T)+C_{P}^{\text {res }}(T, P) \\
C_{V}^{i g}(T)=C_{V}^{t r}+C_{V}^{\text {rot }}+C_{V}^{v i b}=\frac{3}{2} R+\frac{3}{2} R+\sum_{i=1}^{3 N-6} R\left(\frac{\theta_{v i b_{i}}}{T}\right)^{2} \frac{e^{\theta_{v i b_{i}} / T}}{\left(e^{\theta_{v b_{i}} / T}-1\right)^{2}} \\
h_{\text {sens }}^{i g}-R T=\int_{298.15 K}^{T} C_{P}^{i g}(\tau) d \tau-R T \\
C_{P}^{\text {res }}(T, P)=\left(\frac{\partial\left\langle H^{r e s}(T, P)\right\rangle}{\partial T}\right)_{P}=\frac{\partial}{\partial T}\left(P E^{i n t e r}(T, P)+\frac{P}{\rho(T, P)}\right)_{P}
\end{gathered}
$$

In equation (12), $R$ is the universal gas constant, $C_{V}^{\text {tr }}, C_{V}^{\text {rot }}$, and $C_{V}^{v i b}$ are the translational, rotational, and vibrational components of the specific heat at constant volume respectively, $N$ is the number of atoms in the molecule, and $\theta_{v i b_{i}}$ is the characteristic vibrational temperature of the $i^{\text {th }}$ vibrational normal mode. This equation is valid for non-linear molecules. For linear molecules, $C_{V}^{\text {rot }}$ is equal to $R$ and the summation for $C_{V}^{\text {vib }}$ goes from 1 to $3 \mathrm{~N}-5$. A harmonic oscillator is assumed for the 
vibrational contribution to specific heat. In equation (14), $P E^{\text {inter }}$ is the intermolecular potential energy calculated from molecular dynamics simulation, $P$ is the input pressure for the molecular dynamics simulation, and $\rho$ is the resulting density from the molecular dynamics simulation. The intermolecular potential energy from molecular dynamics simulation is found by summing the last two terms of equation (2)

Molecular dynamics simulations of the model compound are performed to determine the intermolecular coefficient values $(\sigma$ and $\varepsilon$ ) necessary to accurately predict the density and enthalpy of the model compound (Figure 3.3). Density predictions are performed solely with molecular dynamics simulations. Enthalpy and entropy predictions are performed with a combination of molecular dynamics simulations and quantum mechanically calculated ideal gas enthalpy and specific heat.

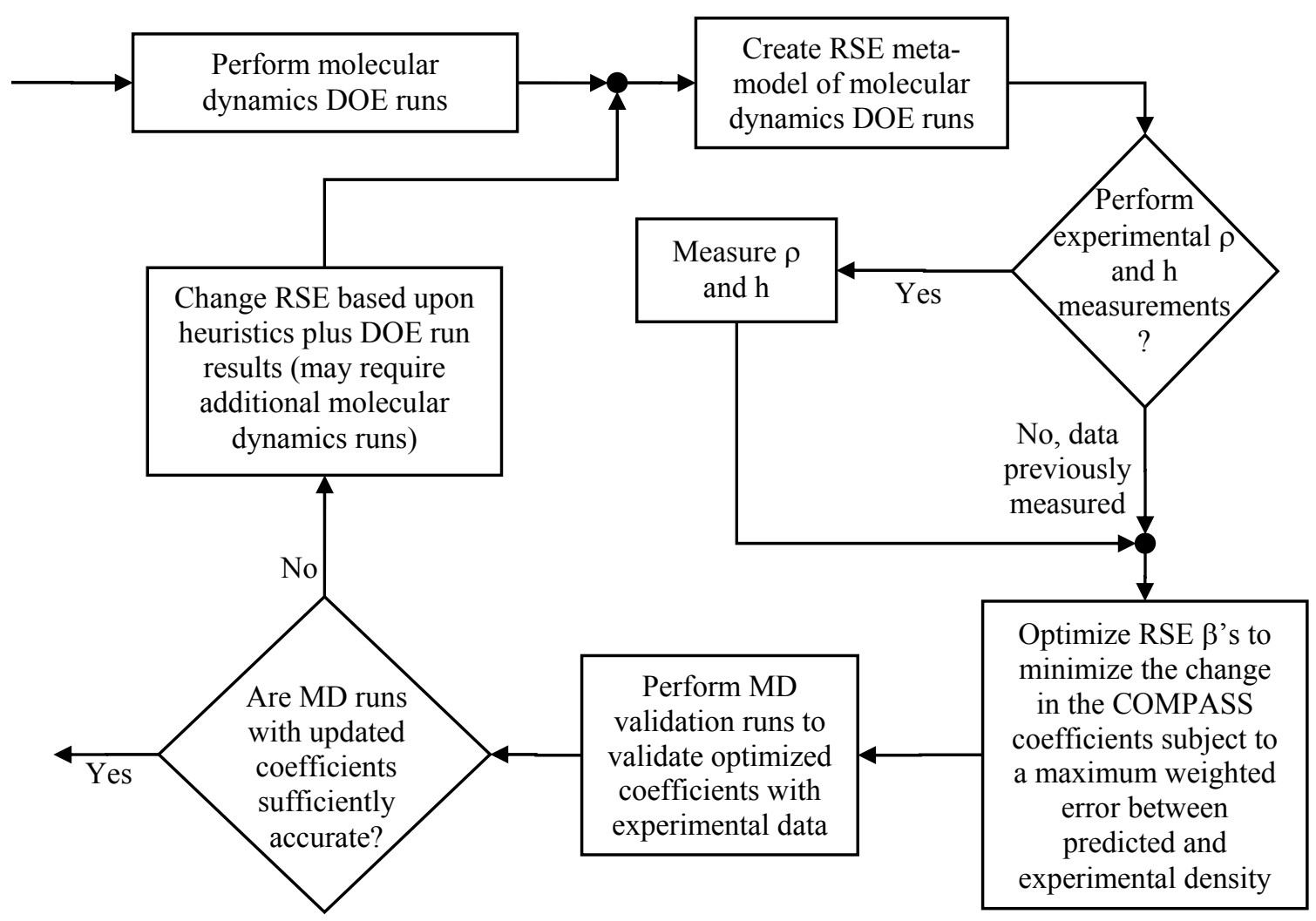

Figure 3.3: Part II: Molecular Dynamics 
The updated intermolecular coefficient values from the model compound are used to perform molecular dynamics simulations of the HEDM compound at appropriate temperature and pressure combinations for rocket engine analysis. Density, enthalpy, and entropy predictions are performed in the same manner as with the model compound.

The HEDM compound density predictions and molecular configuration are used to perform additivity method calculations of kinematic viscosity and thermal conductivity (Figure 3.4).

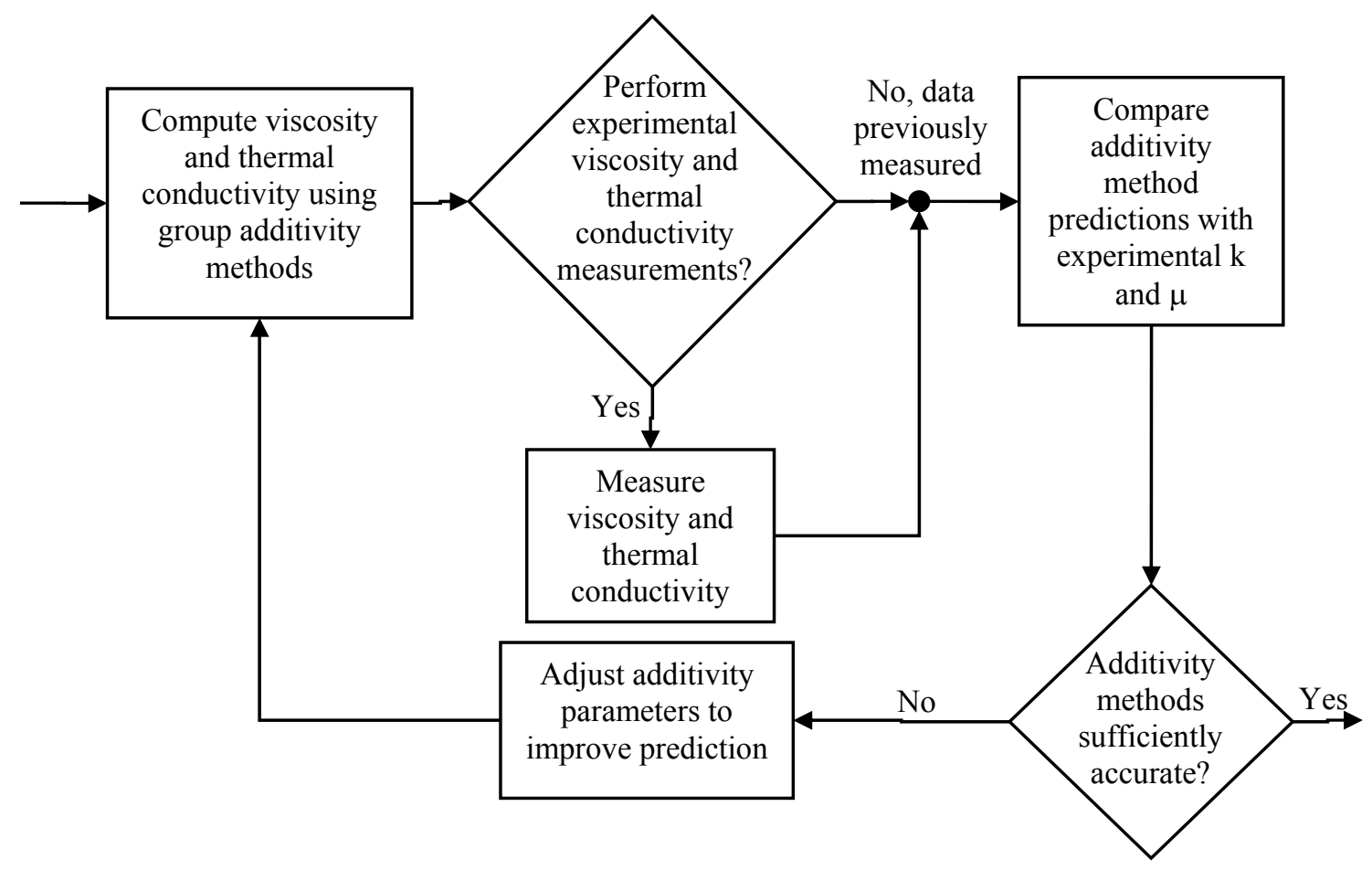

Figure 3.4: Part III: Additivity Methods

\subsection{QUANTUM MECHANICAL ANALYSIS}

Quantum mechanical analyses of both the model compound and corresponding HEDM compound are performed in order to determine each compound's ideal gas enthalpy and specific heat as a function of temperature. As previously discussed, the method developed by Lagache et al. [40] and Cadena et al. [41,42] breaks down the 
calculation of liquid specific heat and enthalpy into ideal gas and residual components. The calculation of the ideal gas component is discussed in this section.

\subsubsection{Determining the Minimum ENERGy Molecular Configuration}

In order to perform both molecular dynamics simulations and normal mode vibrational frequency calculations, the optimized geometry (i.e., global minimum energy configuration) of the molecule of interest is needed. This geometry is found through a two step process. First, a molecular dynamics conformational search of the different dihedral angles found in the molecule is performed. A conformational search is a technique for determining all the different dihedral angles that produce a local minimum. This search method typically uses some domain spanning technique (such as a grid search, simulated annealing, or genetic algorithm) to find the approximate global minimum energy configuration.

Second, the approximate global minimum energy configuration found from this conformational search is used as an initial configuration (starting point) for a quantum mechanical energy minimization in order to find the global minimum energy configuration. A gradient-based optimizer is used with a quantum mechanical energy calculation routine to adjust the molecular configuration in order to find the true global minimum energy configuration. In this work, we employ an industry-standard, quantum chemistry package developed at Iowa State University called the General Atomic and Molecular Electronic Structure System (GAMESS) [43]. This program is chosen because it is freely available, able to perform all the necessary computations, and easy to use.

A notional model of this process is shown in Figure 3.5. The red x's represent the approximate local minimum energy configurations found using the domain-spanning method. The red circled $\mathrm{x}$ represents the approximate global minimum energy configuration. The green plus represents the true global minimum energy configuration found by applying the gradient-based optimization. 


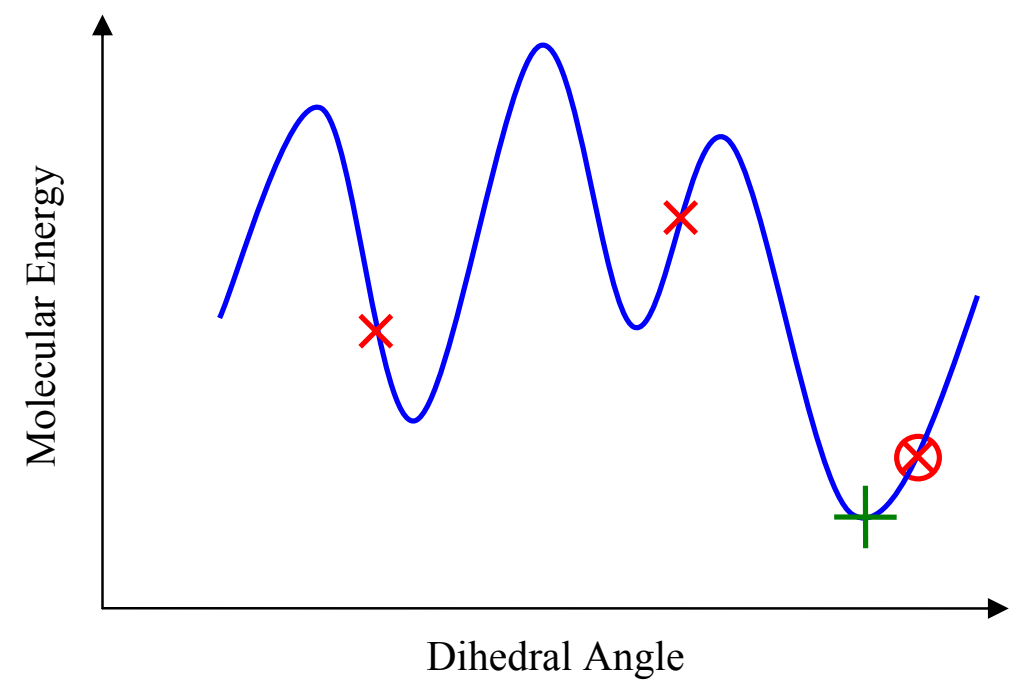

Figure 3.5: Notional Molecular Energy vs. Dihedral Angle

For this research work, GAMESS uses the density functional theory (DFT) energy calculation method with the B3LYP functional and the 6-31G* basis set [44]. DFT is a method used to calculate the ground-state electronic energy of a molecule [45, p.573]. A functional is a rule that takes functions as its argument [45, pp.573-574]. In DFT, a functional represents the link between a molecule's electronic energy and the location of its electrons. A basis set is a set of functions used in quantum chemistry calculations to represent the atomic and molecular orbitals [45]. The $6-31 \mathrm{G}^{*}$ basis set has been shown to be effective for use in the creation of molecular dynamics force fields [46]. Upon the recommendation of Prof. David Sherrill in the Georgia Tech School of Chemistry \& Biochemistry, the combination of DFT with the B3LYP functional and 6-31G* basis set was used for all quantum mechanical energy calculations [47].

\subsubsection{CALCULATION OF IDEAL GAS ENTHALPY AND SPECIFIC HEAT}

With the global minimum energy configuration known, a quantum mechanical vibrational analysis can be performed in order to calculate the vibrational normal mode frequencies of the molecule of interest. A harmonic vibrational analysis is performed 
using GAMESS with the resulting harmonic vibrational frequencies scaled to account for anharmonic effects [48]. Quantum mechanically calculated harmonic vibrational frequencies are generally larger than the vibrational frequencies observed experimentally [49]. As a result, a scale factor (or multiplication factor) is typically used to adjust the calculated vibrational frequencies to better match those observed experimentally. This scale factor, which depends on the energy calculation method and basis set used, is generally between 0.89 and 1.00 [48]. For DFT with the B3LYP functional and 6-31G* basis set, this scale factor is recommended to be 0.9989 [48].

With the set of normal mode vibrational frequencies calculated by a quantum mechanical vibrational analysis and scaled for anharmonic effects, we can calculate the vibrational contribution to the ideal gas specific heat. The following equations are used for a non-linear molecule:

$$
\begin{gathered}
C_{V}^{v i b}(T)=\sum_{i=1}^{3 N-6} R\left(\frac{\theta_{v i b_{i}}}{T}\right)^{2} \frac{e^{\theta_{v i b_{i}} / T}}{\left(e^{\theta_{v i b_{i}} / T}-1\right)^{2}} \\
\theta_{v i b_{i}}=\frac{h v_{i}}{k_{B}}
\end{gathered}
$$

In equation (16), $h$ is Planck's constant, $k_{B}$ is Boltzmann's constant, and $v_{i}$ is the $i^{\text {th }}$ normal mode vibrational frequency found from quantum mechanical analysis. The vibrational frequencies in equation (16), $v_{i}$, are the scaled normal mode vibrational frequencies from quantum mechanical calculations. Using equation (12), we can calculate the ideal gas specific heat as a function of temperature for our molecule of interest. Ideal gas sensible enthalpy (less RT) is calculated by integrating the ideal gas specific heat in the following manner:

$$
h_{\text {sens }}^{i g}-R T=\int_{298.15 K}^{T} C_{P}^{i g}(\tau) d \tau-R T
$$


A validation of this ideal gas calculation method was performed on benzene to verify that this method can accurately calculate the ideal gas specific heat of a molecule over a range of temperatures. Figure 3.6 is a graph of benzene's ideal gas specific heat at constant volume as a function of temperature. The blue line is the experimental ideal gas $\mathrm{C}_{\mathrm{V}}$ taken from the NASA Thermobuild Database [50]. The red line is the quantum mechanically calculated ideal gas $\mathrm{C}_{\mathrm{V}}$ found using equation (12).

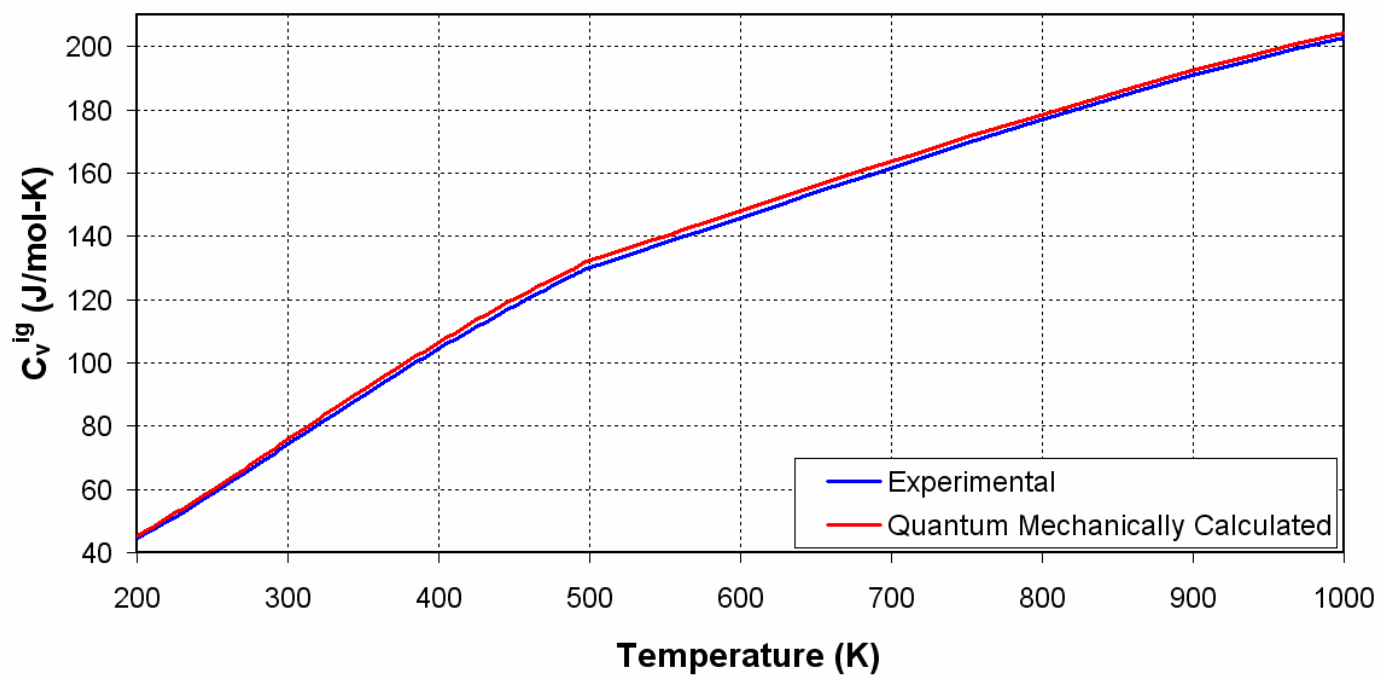

Figure 3.6: Benzene Ideal Gas $C_{V}$ vs. Temperature

The average deviation of the quantum mechanically calculated ideal gas $\mathrm{C}_{V}$ over the temperature range from $200-1000 \mathrm{~K}$ is $1.88 \%$. This deviation is acceptable for application of this method to enthalpy and entropy predictions of rocket engine propellants. If more precise predictions of the ideal gas $C_{V}$ are needed, improved predictions of the normal mode vibrational frequencies can be made using more complex basis sets and functionals [45, pp.588-589, 48].

\subsection{Molecular Dynamics ANAlysis}

Molecular dynamics simulations are used for two purposes: 1) determination of the optimized values for the intermolecular parameters of the COMPASS force field 
model (last two terms in equation (2)), and 2) calculation of the enthalpy, entropy, and density of the HEDM molecule of interest over the entire range of temperature and pressure experienced in a liquid rocket engine.. The COMPASS (Condensed-phase Optimized Molecular Potentials for Atomistic Simulation Studies) model, as the name implies, is a molecular force field model that is optimized for condensed-phases $[8,51,52,53]$. This model is particularly useful for predicting thermophysical properties for rocket propellants because the propellant is normally in a condensed phase for the majority of the time it flows through the different engine components. Figure 3.7 shows a snapshot of a molecular dynamics simulation of one such potential rocket propellant and HEDM molecule, liquid DMAZ. The blue atoms are nitrogen, the cyan atoms are carbon, and the white atoms are hydrogen.

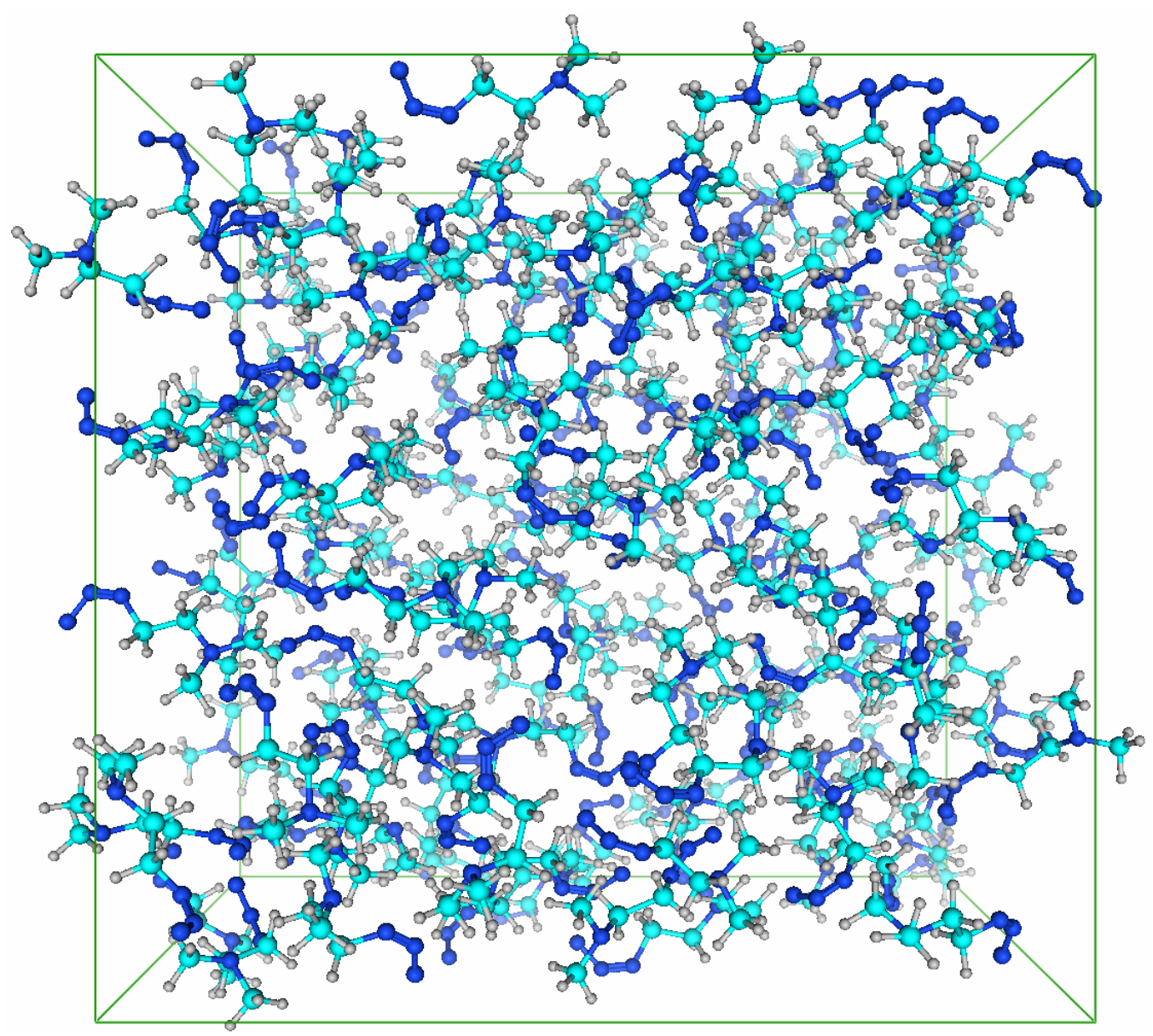

Figure 3.7: Molecular Dynamics Snapshot of Liquid DMAZ 
Molecular dynamics is used initially to determine the appropriate values for the parameters used in the intermolecular terms of the COMPASS model. These terms describe the intermolecular potential (potential between two atoms in different molecules or two atoms in the same molecule not connected through a bond, angle, or dihedral).

Molecular dynamics is used in conjunction with experimental data to determine these parameter values. Due to the fact that little or no experimental data is known for the HEDM propellant of interest, a model compound is used in this step of the method. A model compound should be chosen based on the following rules:

\section{Rule 1: The model compound must have a "similar" molecular structure as the corresponding HEDM compound.}

In this rule, "similar" refers to a comparable molecular geometry and bond structure. For quadricyclane, this means that the model compound must also be a hydrocarbon with a bicyclic structure. For DMAZ, the model compound must have an azide chain (three nitrogen atoms bonded together).

\section{Rule 2: $\quad$ The model compound must have the same atom types as the HEDM compound.}

Atom types are defined in molecular dynamics as an element plus some distinguishing bonding characteristics [8]. For instance, quadricyclane (see Figure 3.8) is defined by three types: two carbon atom types and one hydrogen atom type. The hydrogen atom type, denoted $\mathrm{h} 1$, is a nonpolar hydrogen. The first carbon atom type, denoted $\mathrm{c} 4$, is a generic $\mathrm{sp}^{3}$ carbon. The top carbon atom in Figure 3.8 is a c4 carbon. The second carbon atom type, denoted $\mathrm{c} 43$, is a $\mathrm{sp}^{3}$ carbon with three heavy atoms attached. The remaining carbon atoms in Figure 3.8 are c 43 carbons. 


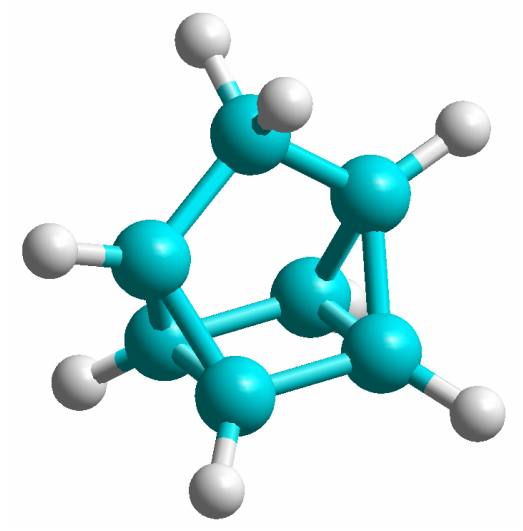

Figure 3.8: Ball and Cylinder Rendering of Quadricyclane $\left(\mathrm{C}_{7} \mathrm{H}_{8}\right)$

\section{Rule 3: The model compound must have some density and enthalpy (or specific heat) experimental data in the liquid phase.}

This rule is necessary because the intermolecular parameters are optimized in order to match the experimentally measured density and specific heat in the liquid phase.

Armed with a model compound chosen based upon these rules, molecular dynamics simulations of the model compound are compared with the corresponding experimental data.

Once the intermolecular parameters have been optimized, they are used for molecular dynamics simulations of the HEDM compound at temperatures and pressures experienced in a liquid rocket engine. Enthalpy change, entropy change, and density are computed at each temperature and pressure combination. These results can be imported into liquid rocket engine powerhead design codes and then used to perform conceptual engine designs using these HEDM propellants.

A potential drawback of this technique of using the optimized intermolecular parameters on a wide temperature and pressure range of molecular dynamics simulations of HEDM compounds is that the parameter optimization is based upon DOE runs at a single liquid temperature and pressure (typically $298 \mathrm{~K}$ and $1 \mathrm{~atm}$ ). An assumption is 
made that one can optimize the intermolecular parameters for a single temperature and pressure combination, and then use those optimized parameters for molecular dynamics simulations at significantly different temperature and pressure combinations.

Molecular dynamics simulations of benzene using the baseline COMPASS model were performed over a wide range of pressures to examine the ability of the COMPASS parameters, optimized for the prediction of properties of liquid compounds at $298 \mathrm{~K}$ and 1 atm., to accurately predict thermophysical properties at vastly different pressures. The results of these simulations are provided in Figure 3.9-Figure 3.11. The results indicate that molecular dynamics can accurately predict the thermophysical properties of substances even when the simulated pressure is significantly different than the one used to optimize the intermolecular potential parameter values. Adding the enthalpy and entropy of formation of liquid benzene to the sensible enthalpies and entropies calculated from the molecular dynamics simulations, the average RMS deviations for density, total enthalpy, and total entropy are provided in Table 3.1. The enthalpy and entropy of formation of liquid benzene are $11.81 \mathrm{kcal} / \mathrm{mol}$ and $41.57 \mathrm{cal} / \mathrm{mol}-\mathrm{K}$ respectively [39].

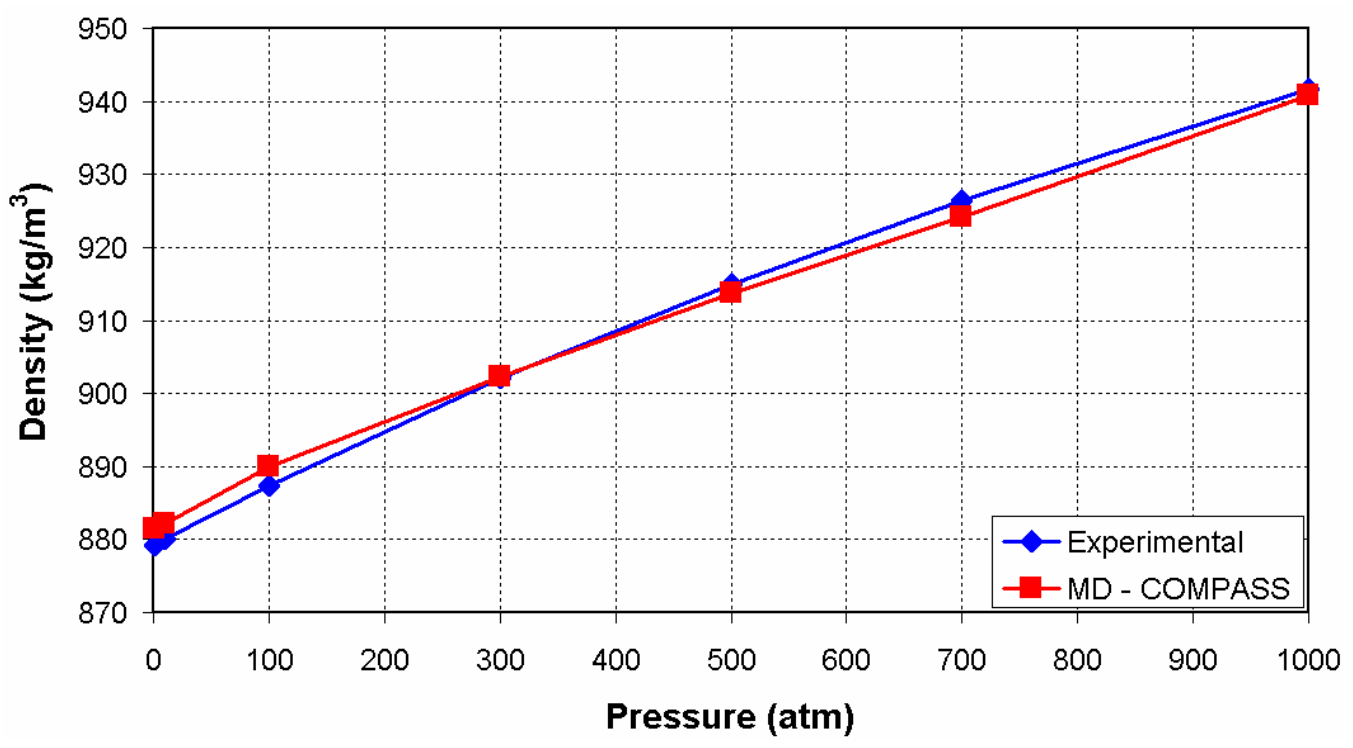

Figure 3.9: Benzene Density vs. Pressure $(\mathrm{T}=298$ K) 


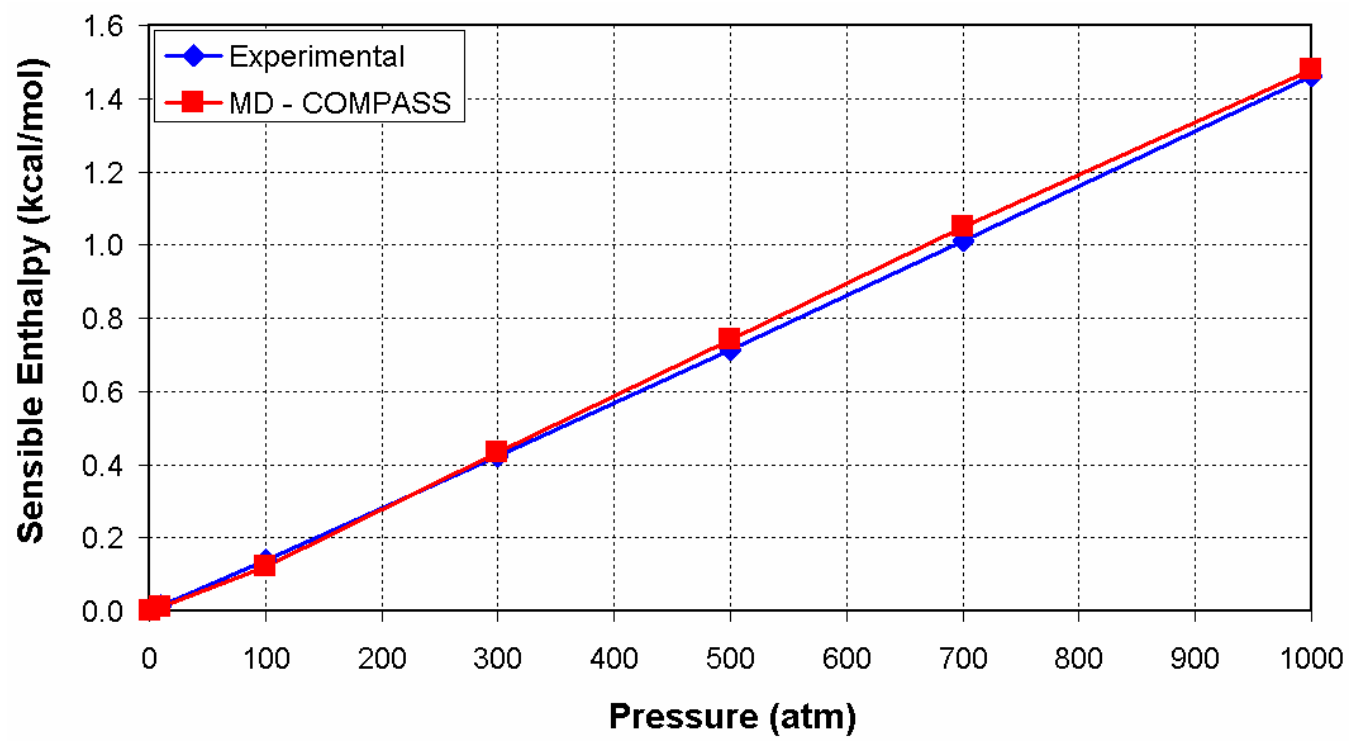

Figure 3.10: Benzene Sensible Enthalpy vs. Pressure $(T=298 \mathrm{~K})$

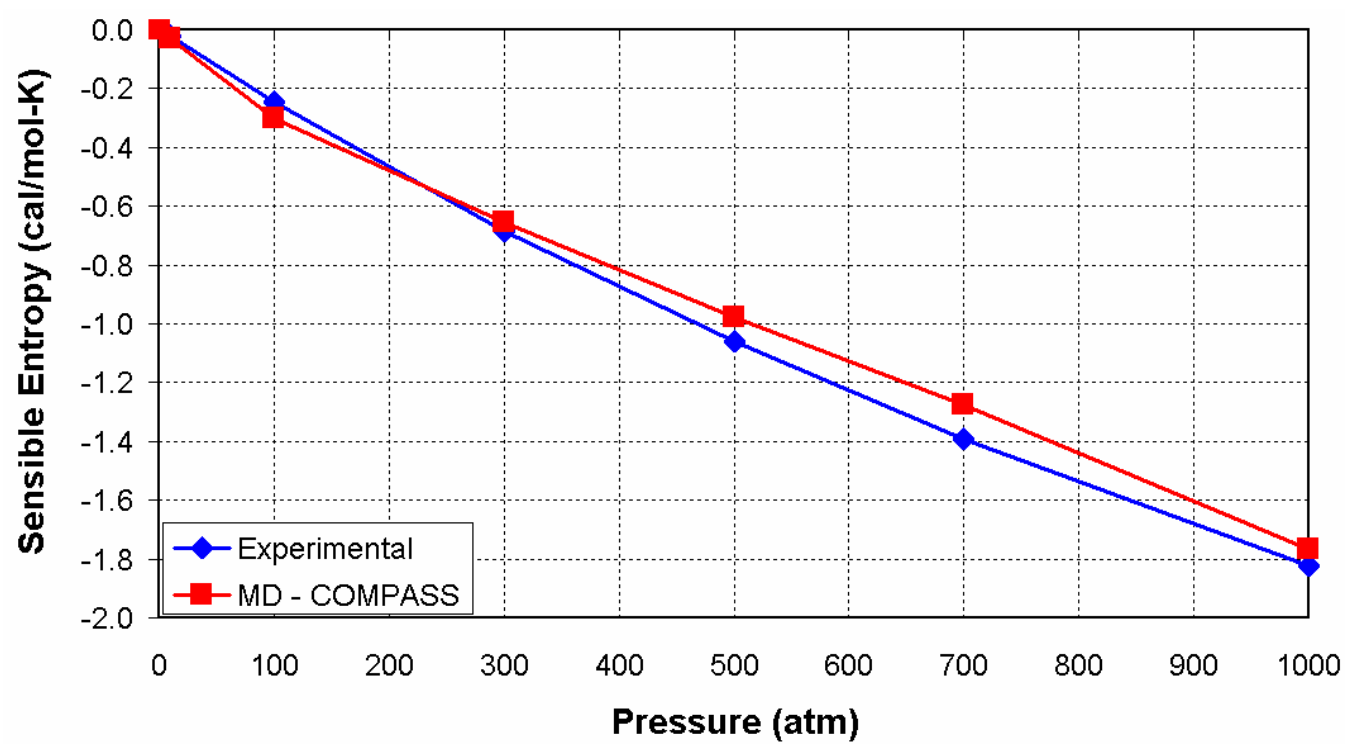

Figure 3.11: Benzene Sensible Entropy vs. Pressure $(T=298$ K)

Table 3.1: Average RMS Deviations of MD Benzene Property Predictions

\begin{tabular}{lc}
\hline & COMPASS Average RMS Deviation \\
\hline Density & $0.18 \%$ \\
Total Enthalpy & $0.13 \%$ \\
Total Entropy & $0.12 \%$ \\
\hline
\end{tabular}


The results shown in Table 3.1 indicate that it is fair to assume that one can use the intermolecular force field parameters optimized at one temperature and pressure for molecular dynamics condensed phase simulations at significantly different pressures.

\subsubsection{DESCRIPTION OF KEY MOLECULAR DYNAMICS ISSUES}

There are several key issues that need to be understood when performing molecular dynamics calculations. These include periodic boundary conditions, longrange cutoffs and corrections terms, time-step sizes and number of time-steps, and the different types of molecular dynamics simulations.

Periodic boundary conditions are a technique of surrounding the computational domain with exact replicas of the computational domain. This technique is useful in simulating thermodynamic properties that are based upon a continuum fluid [31]. Figure 3.12 is a schematic of this idea [10]. The central box is the computational domain and all the surrounding boxes are exact replicas, or images, of the central box.

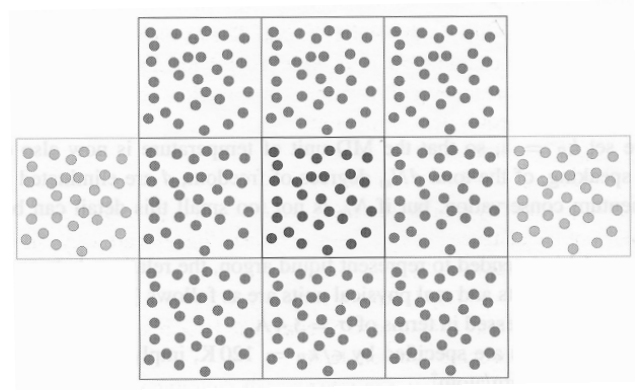

Figure 3.12: Schematic of Periodic Boundary Conditions in Two Dimensions

Long-range cutoffs allow the simulations to avoid detailed calculations of the intermolecular potential between a pair of atoms that are far apart. Long-range cutoffs are used in order to greatly speed up simulations, because all intermolecular potential calculations between two atoms outside the cutoff distance are ignored [54, pp.64-65]. Cutoff distances can be used because the intermolecular potential rapidly approaches zero at large distances (see Figure 2.4). Figure 3.13 is a schematic of the idea of a long-range 
cutoff. The blue atom in the center has a long-range cutoff distance of $r_{\text {LRC }}$ for all intermolecular potential calculations. The intermolecular potentials between the blue atom and the green atoms within the dotted circle are calculated. The intermolecular potentials between the blue atom and the red atoms outside of the dotted circle are not calculated. In this simple case, this reduces the number of intermolecular calculations between the blue atom and the rest of the atoms from seven (four red and three green) to three.

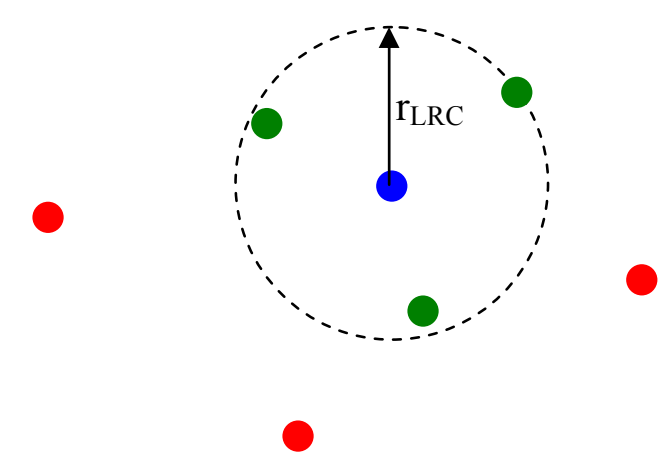

Figure 3.13: Schematic of a Long-Range Cutoff in Two Dimensions

Long-range energy and pressure corrections are used to account for the potential not modeled outside the long-range cutoff distance [31]. For the van der Waals intermolecular potential term (the last term in equation (2)), a simple analytically determined correction is applied to the overall energy and pressure of the system. The equations below are the long-range energy and pressure corrections to the van der Waals potential energy [8].

$$
\begin{gathered}
E_{L R C}=\frac{1}{2} \sum_{i=1}^{n} N_{i} \sum_{j=1}^{n} \rho_{j} 4 \pi \int_{r_{L R C}}^{\infty} E_{i j}(r) r^{2} d r \\
P_{L R C}=\frac{1}{6} \sum_{i=1}^{n} \rho_{i} \sum_{j=1}^{n} \rho_{j} 4 \pi \int_{r_{L R C}}^{\infty} r^{3}\left(\frac{d E_{i j}(r)}{d r}\right) d r
\end{gathered}
$$


In the above equations, $n$ is the number of different intermolecular atom types (described earlier in section 3.3) interacting with an intermolecular pair potential $E_{i j}(r)$. $N_{i}$ is the number of atoms of atom type $i$ and $\rho_{i}$ is the density of atom type $i$. The variable $r$ is the distance between the two atoms whose intermolecular pair potential is being calculated.

The long range Coulomb interactions are modeled using the particle-particle, particle-mesh technique $[10,55,38]$. Coulomb interactions are a result of the charges associated with two atoms $i$ and $j$ (term 11 of equation (2)). This technique for modeling Coulomb interactions is an efficient means of modeling these interactions without utilizing a cutoff distance. A cutoff distance was not used for modeling Coulomb interactions due to the fact that these interactions extend a much larger distance than do van der Waals interactions. As seen in term 11 of equation (2), the Coulomb potential is proportional to $\mathrm{r}^{-1}$ as opposed to $\mathrm{r}^{-6}$ for van der Waals interactions (term 12 of equation (2)).

Two different types of molecular dynamics simulations are utilized for this research work. The first type is a constant number of particles $(\mathrm{N})$, constant volume $(\mathrm{V})$, constant temperature (T) simulation. This type of simulation is known as an NVT simulation [54, pp.40-41]. The second type is a constant number of particles, constant pressure (P), constant temperature (NPT) simulation [54, pp.41-42].

For each molecular dynamics run performed for this research work, the following steps were used.

\section{An initial molecular configuration is input (typically a grid of 125 molecules lined up along the three Cartesian axes).}

A study of the prediction of density and intermolecular potential energy as a function of the number of molecules simulated was performed in order to determine the 
appropriate simulation size. The goal is to find the smallest simulation size that can provide accurate results. Figure 3.14 is a graph of density and intermolecular potential energy of liquid benzene as a function of the number of molecules simulated. As can be seen from the graph, the density and intermolecular energy vary significantly at simulation sizes below 125 molecules, but level off at simulation sizes above 125 molecules. From 125 to 343 molecules, density varies by $0.15 \%$ while intermolecular potential energy varies by $0.86 \%$. A smaller simulation size is desired in order to minimize the required computational time. For this study, it is assumed that the simulation size of 125 molecules found for liquid benzene can be used for liquid HEDM compounds as well.

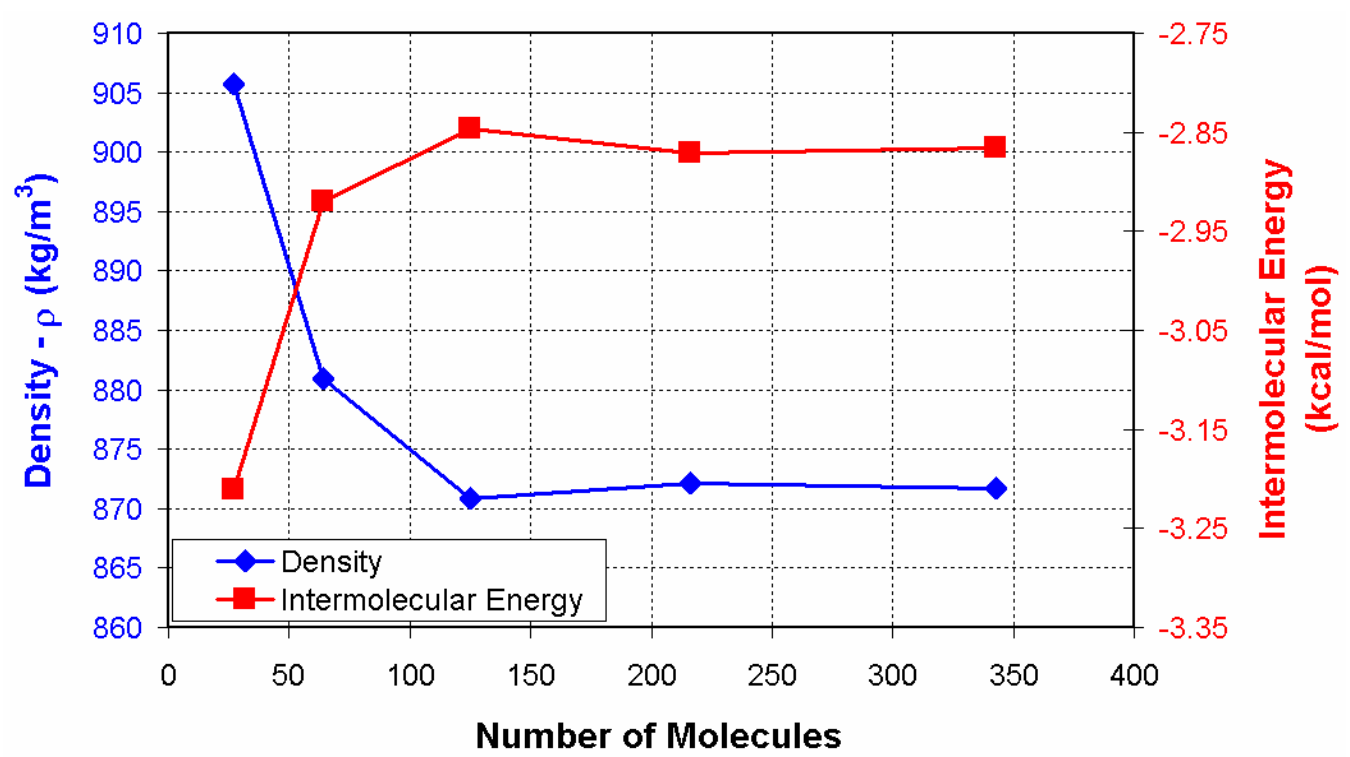

Figure 3.14: Benzene Molecular Dynamics Scaling Issue: $T=300 \mathrm{~K}, \mathrm{P}=1 \mathrm{~atm}$

2. An NVT simulation is then run at the desired temperature and input density using a very small time-step ( $0.1 \mathrm{fs})$ to relieve any pressure gradients that may have been inadvertently introduced into the system with the initial configuration. 
NVT simulations typically require 100,000 time-steps to relieve any initial pressure gradients.

\section{An NPT simulation is then used to gather energy and density data at the desired temperature and pressure. The time-step used for NPT simulations is typically between 0.2 fs and $1.0 \mathrm{fs}$.}

Pressure is not controlled during NVT simulations and is, therefore, generally not the same pressure as that desired during NPT simulations. As a result, at the start of NPT simulations the calculated pressure is substantially different from the desired pressure. Therefore NPT simulations typically require 600,000 to 1 million time-steps to converge to a stable pressure, temperature, and volume. Once convergence has been reached, another 500,000 to 2 million time-steps are run in NPT mode and statistics are collected. Figure 3.15 and Figure 3.16 are graphs showing the convergence history of the calculated density and total enthalpy respectively of a liquid quadricyclane molecular dynamics simulation.

As can be seen from these figures, approximately 600,000 time steps are required for the calculated density to converge to its final solution. Total energy converges more quickly $(\sim 500,000$ time steps). Once convergence has been achieved, both high frequency and low frequency fluctuations still occur. The average of over 1 million time steps is used to compute the density for a particular molecular dynamics simulation. In

the case of Figure 3.15, the average is taken from time step 600,000 to time step 2.2 million. The temperature and energy are also averaged over this simulation period and those results are the ones recorded for use in enthalpy, entropy, and density predictions. 


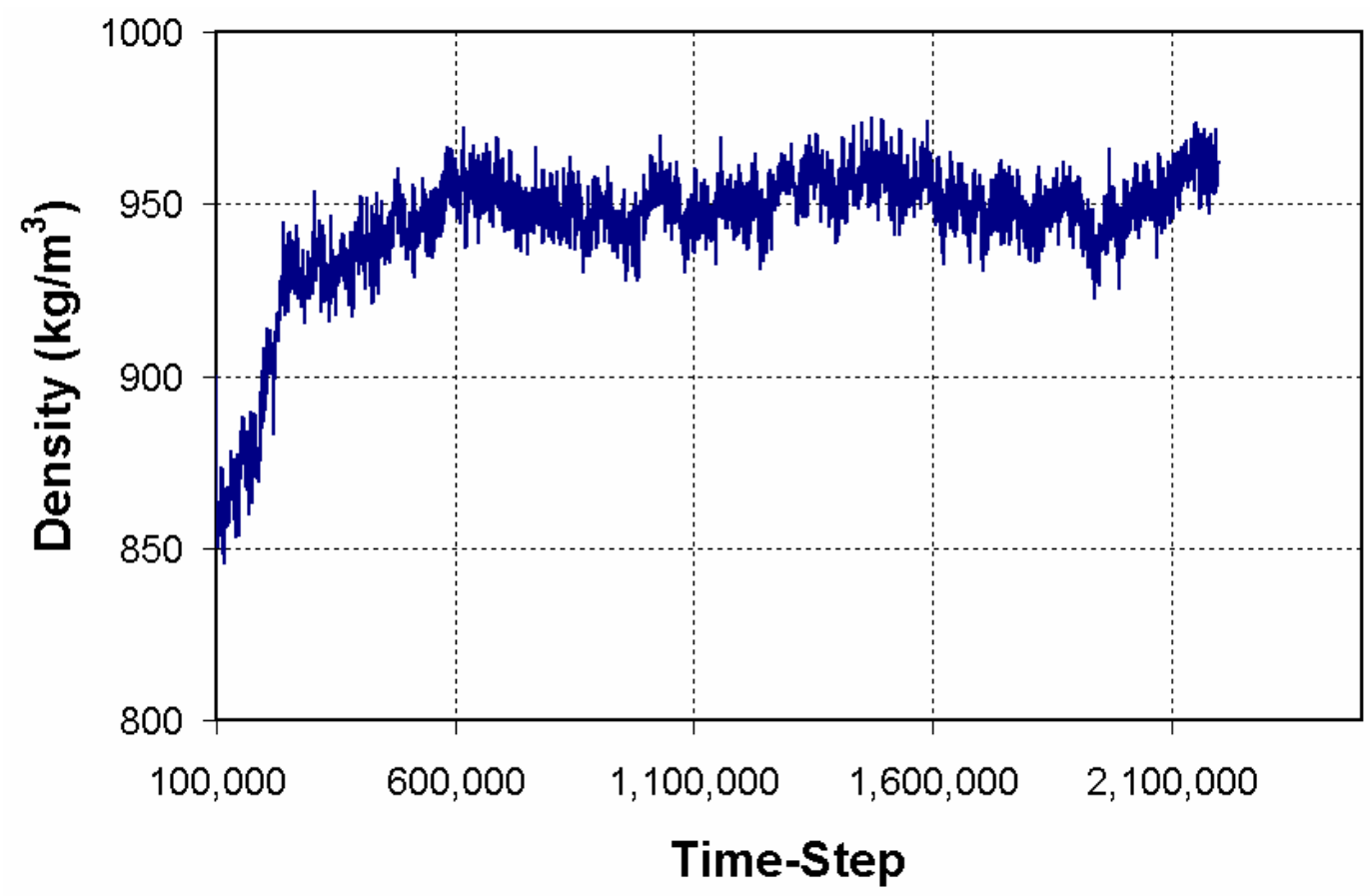

Figure 3.15: Density vs. Time-Step for Quadricyclane

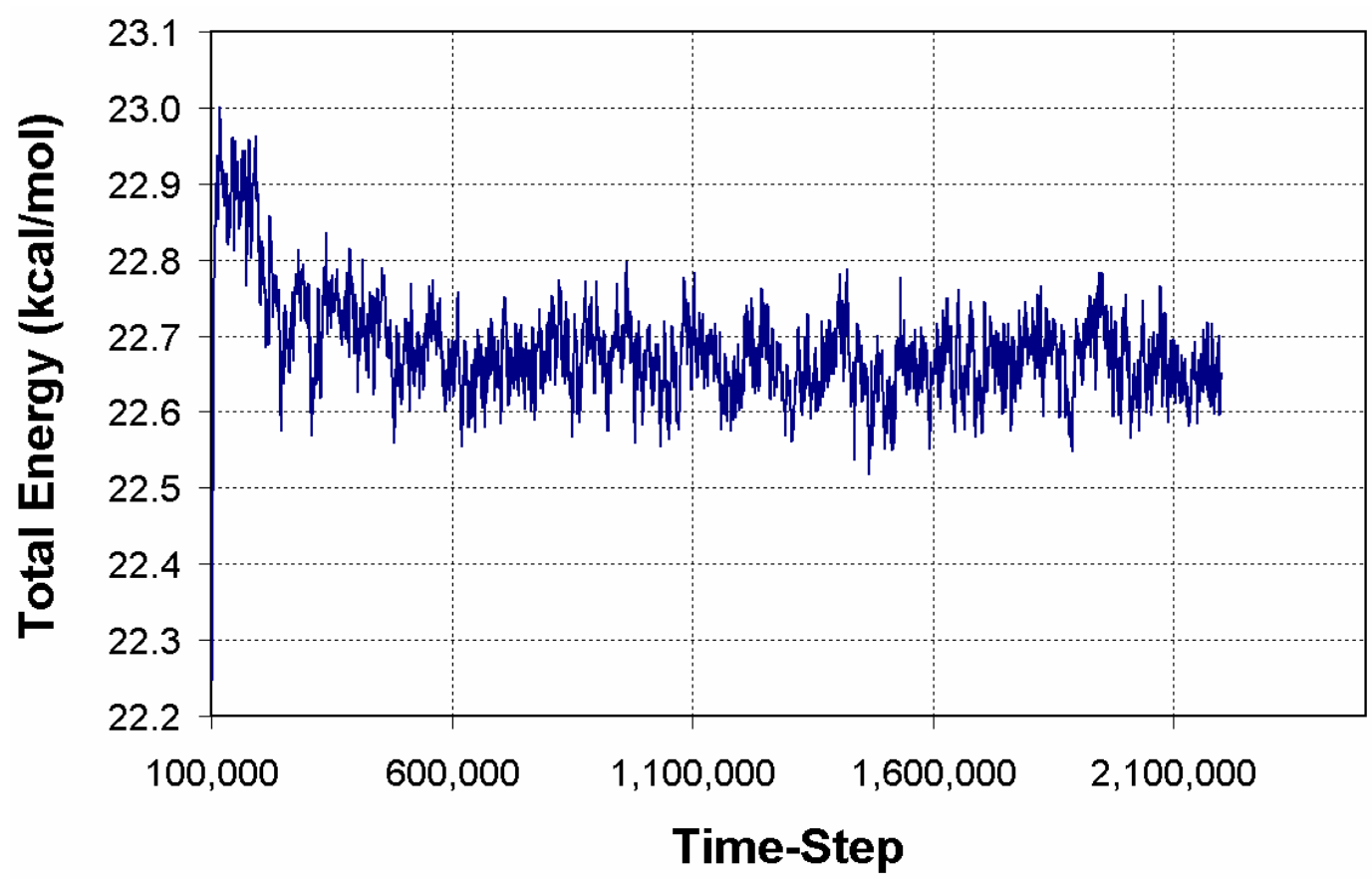

Figure 3.16: Total Energy vs. Time-Step for Quadricyclane 


\subsubsection{Fitting Intermolecular Parameters}

Molecular dynamics is used initially to fit the intermolecular parameters. This is done by comparing the results of MD simulations with experimental data and adjusting the intermolecular parameters of the force field function in order to better match experimental data. For this work, the experimental data of interest is the density and specific heat of the model compound as a function of temperature and pressure.

The intermolecular potential parameters are initially set using a $6^{\text {th }}$ order combination law for unlike atom pairs (two atoms of different atom types) shown in the equations below. Parameter values for like atom pairs (two atoms of the same atom type) are known from published results $[8,9,18,19,51,53]$. Parameter values for unlike atom pairs can be computed very quickly using the equations below.

$$
\begin{gathered}
\sigma_{i j}=\left[\frac{\left(\sigma_{i}\right)^{6}+\left(\sigma_{j}\right)^{6}}{2}\right]^{1 / 6} \\
\varepsilon_{i j}=2 \sqrt{\varepsilon_{i} \varepsilon_{j}}\left[\frac{\left(\sigma_{i}\right)^{3}\left(\sigma_{j}\right)^{3}}{\left(\sigma_{i}\right)^{6}+\left(\sigma_{j}\right)^{6}}\right]
\end{gathered}
$$

In the above equations, $\sigma_{i}$ is the Lennard-Jones-9-6 parameter for atom $i, \sigma_{j}$ is the Lennard-Jones-9-6 parameter for atom $j, \varepsilon_{i}$ is the energy potential well depth for atom $i, \varepsilon_{j}$ is the energy potential well depth for atom $j$, and $\sigma_{i j}$ and $\varepsilon_{i j}$ are combinations for a pair of atoms $i$ and $j$. The two combination parameters $\sigma_{i j}$ and $\varepsilon_{i j}$ are used in the last two terms of the COMPASS model shown in equation (2). The term Lennard-Jones refers to the potential upon which these parameters are used. The Lennard-Jones-9-6 potential, which is the last term of the COMPASS model, can and has been used by itself as a potential function. It is effective in modeling simple molecules and was originally used in the 1960's for the modeling of liquid argon [10]. 


\subsubsection{DESIGN OF EXPERIMENTS}

A design of experiments (DOE) is performed on the intermolecular parameters to determine the sensitivity of density to changes in these parameters. DOE techniques are a structured approach for changing design variable settings (in the case of this work, the design variables are $\sigma_{i j}$ and $\varepsilon_{i j}$ ) and observing the resulting response (in the case of this work, the response is density) [56, pp.549-550]. The DOE used in this work is the central composite design (CCD) [56, pp.645-650].

For a molecule with two atom types, there are four design variables ( $\sigma$ and $\varepsilon$ for each atom type). A four design variable CCD run list results in 27 runs (with three center points) and is shown in Table 3.2 below. The ' 0 ' settings in Table 3.2 represent the baseline value for the particular design variable. The ' +1 ' settings represent the baseline value plus some perturbation. In the case of this molecular dynamics work, this perturbation typically was $10 \%$ of the baseline $\sigma$ or $\varepsilon$ value. The ' -1 ' settings represent the baseline value minus the same perturbation.

The first 16 runs are a full factorial array. The number of runs in a full factorial array is equal $2^{\mathrm{N}}$ where $\mathrm{N}$ equals the number of design variables. Runs 17-24 are the star points. Star points are designs within a CCD which have only a single variable altered from the baseline value. Runs 25-27 are the center points. The center points are designs with all design variable settings set at the baseline values. Multiple center points are used in general when the process or program being run has some variability. In the case of molecular dynamics, the initial velocity vectors of the molecules are computed using a random number generator with a random number seed. The three center points each have a different random number seed and result in slightly different simulation results. The JMP Statistical Discovery Software tool [57] was used to create all DOE run lists used for this research work. 
Table 3.2: Four Design Variable CCD Run List

\begin{tabular}{ccccc}
\hline Run \# & $\sigma_{1}$ & $\varepsilon_{1}$ & $\sigma_{2}$ & $\varepsilon_{2}$ \\
\hline 1 & -1 & -1 & -1 & -1 \\
2 & -1 & -1 & -1 & +1 \\
3 & -1 & -1 & +1 & -1 \\
4 & -1 & -1 & +1 & +1 \\
5 & -1 & +1 & -1 & -1 \\
6 & -1 & +1 & -1 & +1 \\
7 & -1 & +1 & +1 & -1 \\
8 & -1 & +1 & +1 & +1 \\
9 & +1 & -1 & -1 & -1 \\
10 & +1 & -1 & -1 & +1 \\
11 & +1 & -1 & +1 & -1 \\
12 & +1 & -1 & +1 & +1 \\
13 & +1 & +1 & -1 & -1 \\
14 & +1 & +1 & -1 & +1 \\
15 & +1 & +1 & +1 & -1 \\
16 & +1 & +1 & +1 & +1 \\
17 & -1 & 0 & 0 & 0 \\
18 & +1 & 0 & 0 & 0 \\
19 & 0 & -1 & 0 & 0 \\
20 & 0 & +1 & 0 & 0 \\
21 & 0 & 0 & -1 & 0 \\
22 & 0 & 0 & +1 & 0 \\
23 & 0 & 0 & 0 & -1 \\
24 & 0 & 0 & 0 & +1 \\
25 & 0 & 0 & 0 & 0 \\
26 & 0 & 0 & 0 & 0 \\
27 & 0 & 0 & 0 & 0 \\
\hline & & & &
\end{tabular}

With the DOE run list created, molecular dynamics simulations of the model compound are performed using the appropriate intermolecular parameter input values.

\subsubsection{RESPONSE SURFACE EQUATION}

A response surface is created from the molecular dynamics simulation results of the runs created in section 3.3.3. A response surface is a multidimensional curve-fit of some response variable of interest [56, p.643]. Two response surfaces are created to relate the predicted density and predicted specific heat at constant pressure $\left(C_{P}\right)$ from MD 
simulations to the settings for the intermolecular parameters. The response surface equation (RSE) used is a second order response surface which includes all first order effects and all second order effects, including cross terms. For the four design variable example discussed in 3.3.3, this results in the following RSE for density:

$$
\begin{gathered}
\rho=\beta_{\rho 0}+\beta_{\rho 1} \bar{\sigma}_{1}+\beta_{\rho 2} \bar{\varepsilon}_{1}+\beta_{\rho 3} \bar{\sigma}_{2}+\beta_{\rho 4} \bar{\varepsilon}_{2}+\beta_{\rho 5} \bar{\sigma}_{1}^{2}+\beta_{\rho 6} \bar{\varepsilon}_{1}^{2}+\beta_{\rho 7} \bar{\sigma}_{2}^{2}+\beta_{\rho 8} \bar{\varepsilon}_{2}^{2}+ \\
\beta_{\rho 9} \bar{\sigma}_{1} \bar{\varepsilon}_{1}+\beta_{\rho 10} \bar{\sigma}_{1} \bar{\sigma}_{2}+\beta_{\rho 11} \bar{\sigma}_{1} \bar{\varepsilon}_{2}+\beta_{\rho 12} \bar{\varepsilon}_{1} \bar{\sigma}_{2}+\beta_{\rho 13} \bar{\varepsilon}_{1} \bar{\varepsilon}_{2}+\beta_{\rho 14} \bar{\sigma}_{2} \bar{\varepsilon}_{2} \\
\bar{\sigma}_{i}=\frac{2\left(\sigma_{i}-\sigma_{i}^{\min }\right)}{\sigma_{i}^{\max }-\sigma_{i}^{\min }}-1
\end{gathered}
$$

In equation (22), the $\sigma$ and $\varepsilon$ multipliers are normalized values based upon equation (23). These values are normalized based upon the maximum and minimum settings for each multiplier. In the example discussed in 3.3.3, the maximum and minimum values are +1 and -1 . This normalization is performed to help compensate for severe differences in the magnitude of each design variable in the RSE. This enables the user to quickly see the relative importance of a particular term in the RSE by looking at the magnitude of the $\beta_{\mathrm{i}}$ 's.

A similar RSE is created for the predicted specific heat at constant pressure from MD simulation results. In the above equation, the $\beta_{\rho \mathrm{i}}$ values are coefficients calculated from the DOE simulation run results using a least squares fit. These coefficient values are calculated using the JMP software. In this example, 14 coefficients are created using a DOE with 27 runs. JMP also calculates the goodness of fit, or $\mathrm{R}^{2}$ value, for the RSE. The $\mathrm{R}^{2}$ value is a measure of how well the RSE approximates the responses from the DOE runs [56:323]. The equation for $\mathrm{R}^{2}$ is provided below:

$$
R^{2}=1-\frac{S S_{\text {error }}}{S S_{\text {total }}}
$$


In the above equation, $S S_{\text {error }}$ is the sum of squares for error [58, p.542] and $S S_{\text {total }}$ is the total sum of squares [58, p.543]. The closer $\mathrm{R}^{2}$ is to a value of 1 , the better the RSE approximates the molecular dynamics results. The RSEs created for this research work have $\mathrm{R}^{2}$ values between 0.90 and 0.99 .

\subsubsection{OPTIMIZATION AND VALIDATION}

Once the RSEs for density and specific heat are created, the intermolecular variable values are optimized. The goal of the optimization is to match the RSE predicted density and specific heat values with experimental results. The objective function for the optimizer is:

$$
S=\sum_{i=1}^{N}\left(x_{i}-1\right)^{2}
$$

In equation (25), $x_{i}$ is the current value for the multiplier for the $i^{\text {th }}$ intermolecular parameter and $N$ is the number of intermolecular parameters. When the intermolecular parameters are set to their baseline values, the $x_{i}$ 's are all equal to 1 . This optimization is subject to the following constraint:

$$
\begin{aligned}
& g=\sum_{i=1}^{M}\left[W_{\rho_{-} i} \sqrt{\left(\frac{\rho_{\text {predicted_i }}-\rho_{\text {actual }} i}{\rho_{\text {actual }} i}\right)^{2}}+W_{c p_{-} i} \sqrt{\left(\frac{C_{P_{\text {predicted }} i}-C_{P_{\text {actual }} i}}{C_{P_{\text {actual }} i}}\right)^{2}}\right] \\
& -E_{\max } \leq 0
\end{aligned}
$$

In equation (26), $M$ is the number of (T, P) combinations modeled, $\rho_{\text {predicted_i }}$ and $C_{\text {Ppredicted } i}$ are the RSE predicted values for density and specific heat at the $i^{\text {th }}(\mathrm{T}, \mathrm{P})$ combination. The variables $\rho_{\text {actual } \_i}$ and $C_{\text {Pactual } \_i}$ are the experimentally measured values for density and specific heat at the $i^{\text {th }}(\mathrm{T}, \mathrm{P})$ combination. $W_{\rho_{-} i}$ and $W_{c p_{-} i}$ are the weighting factors for the density and specific heat terms respectively for the $i^{\text {th }}(\mathrm{T}, \mathrm{P})$ combination. One may want to optimize the intermolecular parameters so that the 
density is predicted very accurately at the expense of a less accurate prediction of specific heat or optimize the intermolecular parameters so that a particular temperature and pressure combination is predicted more accurately than others. For the first case, one would make the density weighting factor larger than the specific heat weighting factor. For the second case, one would make the weighting factors for the particular temperature and pressure combination of interest larger than for the other combinations. $E_{\max }$ is the maximum allowable weighted error. Typically, the smaller $E_{\max }$ is, the larger the deviation from the baseline intermolecular parameters is required.

The optimized values for the intermolecular parameters are then used in molecular dynamics simulations to validate the new parameter values. This is done to verify that the RSEs did an adequate job at approximating the molecular dynamics results. If the validation runs produce results that do not match the RSE predicted results, additional terms may need to be added to the RSE to improve its approximation of the molecular dynamics results. In this research work, additional terms were not needed to improve the RSE prediction, but may, in general, be needed in some propellant analyses.

\subsubsection{Molecular Dynamics Thermophysical Property Calculations}

Molecular dynamics is used to compute the equilibrium properties of both the model compound and the HEDM compound at a range of temperatures and pressures $[31,10]$. This is done by expressing these equilibrium properties as a function of the positions and momenta of the particles in the system.

The first observable that we define is the kinetic energy per particle. For a system of point masses, the average kinetic energy of the system is defined using equation (27).

$$
E_{k i n}=\frac{1}{2} \sum_{i=1}^{N} m_{i}\left(\vec{v}_{i} \cdot \vec{v}_{i}\right)
$$


In the above equation, $N$ is the number of particles in the system and $m_{i}$ and $\vec{v}_{i}$ are the respective mass and velocity of particle $i$. In the case of polyatomic molecules, $m_{i}$ is the mass of the molecule and $\vec{v}_{i}$ is the velocity of the center of mass of the molecule. From this definition of kinetic energy the average translational temperature of the system of particles (in three-dimensional space) can be defined using equation (28):

$$
T_{\text {trans }}=\frac{2 E_{k i n}}{3 N k_{B}}=\frac{1}{3 N k_{B}} \sum_{i=1}^{N} m_{i}\left(\vec{v}_{i} \cdot \vec{v}_{i}\right)=\frac{2}{3 k_{B}}\left(\frac{1}{2 N} \sum_{i=1}^{N} m_{i}\left(\vec{v}_{i} \cdot \vec{v}_{i}\right)\right)
$$

where $k_{B}$ is Boltzmann's constant. With the translational temperature defined, the pressure can then be defined using equation (29).

$$
P=\frac{N k_{B} T_{\text {trans }}}{V}+\frac{1}{3 V} \sum_{i=1}^{N-1} \sum_{j=i+1}^{N} \sum_{\alpha=1}^{n} \sum_{\beta=1}^{n} \vec{f}_{i j}^{\alpha \beta} \cdot \vec{r}_{i j}^{\alpha \beta}
$$

where $V$ is the volume of the computational space, $\vec{f}_{i j}^{\alpha \beta}$ is the force vector of atom $\beta$ of molecule $j$ on atom $\alpha$ of molecule $i$, and $\vec{r}_{i j}^{\alpha \beta}$ is the position vector from atom $\beta$ of molecule $j$ to atom $\alpha$ of molecule $i$. The first term of equation (29) is the ideal gas term while the second term is the configurational or "virial" term that becomes prominent at higher densities (farther away from the ideal gas assumption). Density is defined as:

$$
\rho=\frac{N}{V} \sum_{i=1}^{N} m_{i}
$$

With these fundamental properties defined as a function of the positions and momenta of the particles, more complex equilibrium properties can also be defined. For example, the definition of enthalpy begins by dividing it into three components: enthalpy of formation and ideal gas sensible enthalpy and residual sensible enthalpy, 


$$
\begin{gathered}
h(T, P)=\Delta h_{f}^{0}+h_{\text {sens }}^{\text {ig }}(T)+h_{\text {sens }}^{\text {res }}(T, P) \\
h_{\text {sens }}^{\text {res }}(T, P)=\left[E_{\text {inter }}(T, P)+\frac{P}{\rho}\right]-\left[E_{\text {inter }}\left(T_{0}, P_{0}\right)+\frac{P_{0}}{\rho_{0}}\right] \\
E_{\text {inter }}(T, P)=E_{\text {vdwl }}(T, P)+E_{\text {coul }}(T, P)
\end{gathered}
$$

In equation (31), the enthalpy of formation, $\Delta h_{f}^{0}$, is typically defined as the enthalpy of the substance at $298 \mathrm{~K}$ and $1 \mathrm{~atm}$. with respect to the arbitrary basis elements that make up that particle at the same temperature and pressure [24]. The second and third terms in equation (31) are defined using the method developed by Lagache [40] and Cadena et al. [41,42] for calculating the enthalpy of a liquid substance using quantum mechanics and molecular dynamics.

The second term, $h_{\text {sens }}^{\text {ig }}$, is the ideal gas sensible enthalpy defined previously in equation (17). The ideal gas sensible enthalpy is based upon the normal mode vibrational frequencies found from quantum mechanical analysis. The third term in equation (31), the residual sensible enthalpy, $h_{\text {sens }}^{\text {res }}$, represents the condensed phase influence on the sensible enthalpy. This term, found from molecular dynamics simulations, is based upon the intermolecular energy (van der Waals and Coulombic) and the pressure divided by the density. This method for calculating enthalpy is different from that suggested by others $[10,54]$ that attempts to calculate enthalpy using solely molecular dynamics simulations. In this research work, it was found that the method developed by Lagache and Cadena resulted in a more accurate prediction of specific heat, enthalpy, and entropy than the method using only molecular dynamics.

Due to the fact that the intramolecular potential energy calculation (calculated from the quantum mechanical analysis) and the intermolecular potential energy calculation (calculated from molecular dynamics) are performed separately, any influence 
of one potential on the other is not captured using this method [40,41,42]. As a result, a study was performed in order to capture of the influence of the intermolecular potential energy on the intramolecular potential energy.

A series of NVT (constant number of particles, constant volume or density, constant temperature) molecular dynamics simulations of benzene were performed and the computed intermolecular and intramolecular energies were studied. The COMPASS potential model was used for both intermolecular and intramolecular energies. Figure 3.17 is a graph of van der Waals intermolecular energy and total intramolecular energy as a function of density for molecular dynamics simulations of benzene.

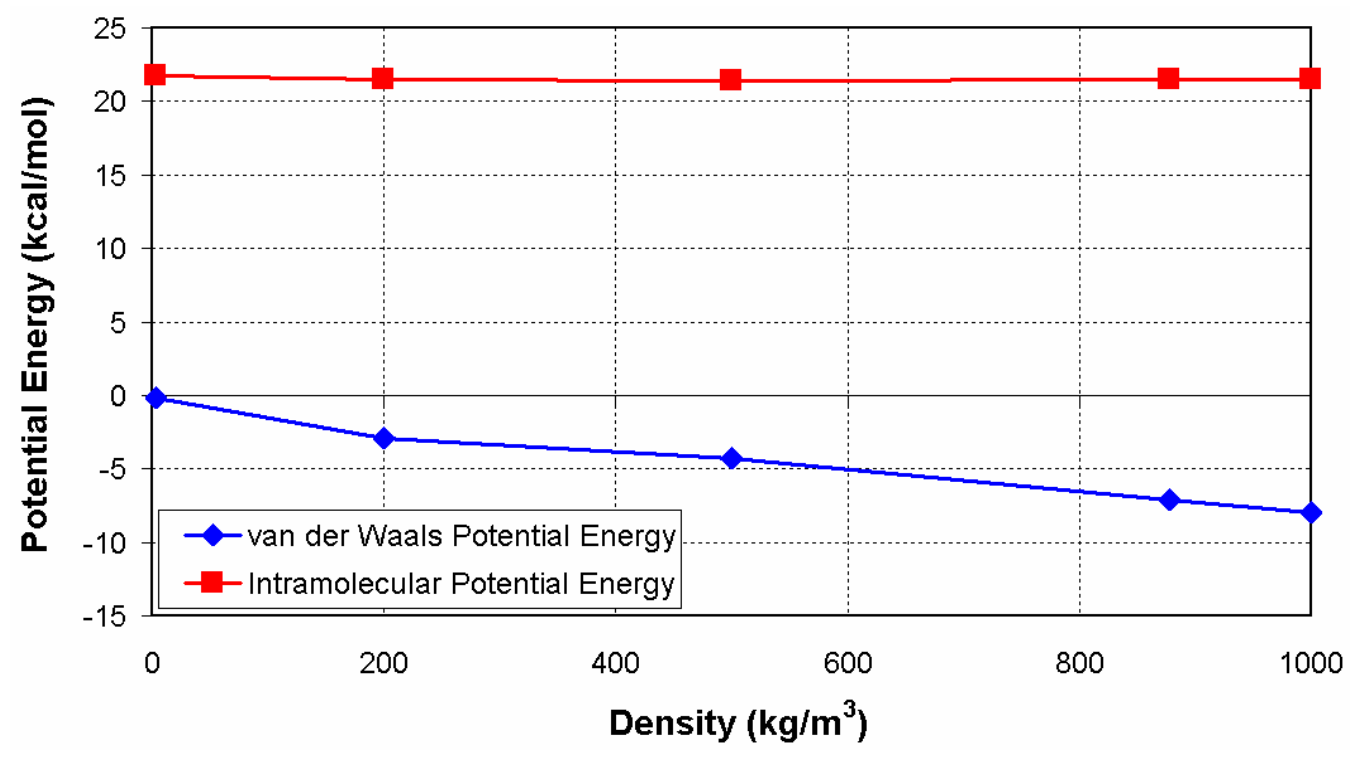

Figure 3.17: Benzene Potential Energy Interaction Issue

The results indicate that the intermolecular potential has very little influence on the intramolecular potential (the intramolecular potential energy line is fairly flat over a wide range of densities while the intermolecular potential energy varies significantly). Over the range of densities studied the intramolecular potential energy changes by $1.56 \%$. This insensitivity of intramolecular potential to changes in intermolecular potential indicates that the calculations of the two potentials can be performed separately with 
minimal error [59] and thus the method developed by Lagache et al. [40] and Cadena et al. $[41,42]$ can be used in the prediction of enthalpy and specific heat.

The second equilibrium property defined is entropy. Entropy can be defined similar to enthalpy by breaking up entropy into its two major components: entropy of formation and sensible entropy:

$$
s(T, P)=\Delta s_{f}^{1 a t m}+s_{\text {sens }}(T, P)
$$

The entropy of formation, $\Delta s_{f}^{1 a t m}$, is typically defined as the entropy of the substance at $298 \mathrm{~K}$ and $1 \mathrm{~atm}$. The entropy of a substance is normally defined to be zero at $0 \mathrm{~K}$. The second term, $s_{\text {sens }}$, is known as the sensible entropy and is defined as the difference in entropy between any given state and the state at which the formation entropy is defined. The entropy of formation of the molecule of interest is taken from the quantum mechanics energy calculation code GAMESS. The sensible entropy for a simple compressible substance is defined with the following "T ds" relation [60, p.236]:

$$
T * d s=d h-v^{*} d P=d h-\frac{d P}{\rho}
$$

Dividing through by the temperature, we get:

$$
d s=\frac{d h}{T}-\frac{d P}{\rho T}
$$

Using the chain rule [61, p.944], $d s$ can be broken up into temperature and pressure differentials with the following equation:

$$
d s=\frac{\partial h /\left.\partial T\right|_{P} d T}{T}+\frac{\partial h /\left.\partial P\right|_{T} d P}{T}-\frac{d P}{\rho T}=\left[\frac{\partial h /\left.\partial T\right|_{P}}{T}\right] d T+\left[\frac{\partial h /\left.\partial P\right|_{T}}{T}-\frac{1}{\rho T}\right] d P
$$


Due to the fact that the definition of the change in entropy from one state (temperature and pressure point) to another is path independent [62, pp.33-36], we define $S_{\text {sens }}\left(T_{1}, P_{1}\right)$ with the following equation:

$$
s_{\text {sens }}\left(T_{1}, P_{1}\right)=\left[s\left(T_{1}, P_{0}\right)-s\left(T_{0}, P_{0}\right)\right]+\left[s\left(T_{1}, P_{1}\right)-s\left(T_{1}, P_{0}\right)\right]
$$

where $T_{0}$ and $P_{0}$ are the temperature and pressure at which the entropy of formation is defined $(298 \mathrm{~K}$ and $1 \mathrm{~atm})$ and $s\left(T_{0}, P_{0}\right)$ is the entropy of formation, $\Delta \mathrm{s}_{f}^{1 \text { atm }}$. Figure 3.18 is a graphical representation of the process used for calculating sensible enthalpy. With enthalpy defined at $\mathrm{T}_{0}$ and $\mathrm{P}_{0}$, the entropy change is first calculated for a constant pressure process from $\left(\mathrm{T}_{0}, \mathrm{P}_{0}\right)$ to $\left(\mathrm{T}_{1}, \mathrm{P}_{0}\right)$. The entropy change is then calculated for a constant temperature process from $\left(\mathrm{T}_{1}, \mathrm{P}_{0}\right)$ to $\left(\mathrm{T}_{1}, \mathrm{P}_{1}\right)$, the desired temperature and pressure.

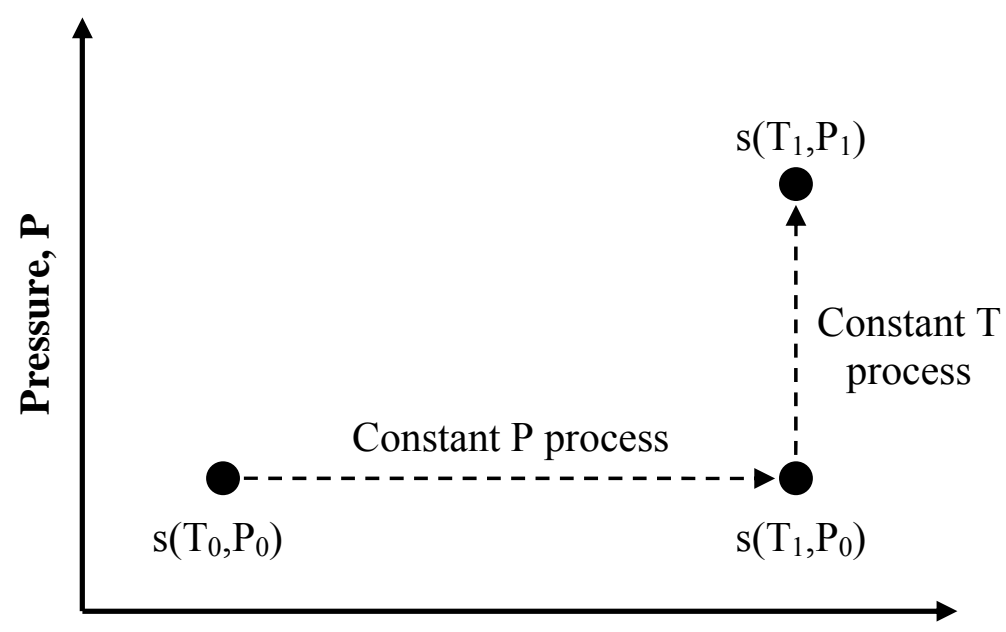

Temperature, $\mathrm{T}$

Figure 3.18: Process of Calculating Entropy Change

Using equation (37), the constant pressure process from $\left(\mathrm{T}_{0}, \mathrm{P}_{0}\right)$ to $\left(\mathrm{T}_{1}, \mathrm{P}_{0}\right)$ is defined as follows [63, p.534]: 


$$
s\left(T_{1}, P_{0}\right)-s\left(T_{0}, P_{0}\right)=\int_{T_{0}}^{T_{1}} d s=\int_{T_{0}}^{T_{1}}\left[\frac{\partial h /\left.\partial T\right|_{P}}{T}\right] d T=\int_{T_{0}}^{T_{1}} \frac{C_{P}\left(T, P_{0}\right)}{T} d T
$$

The constant temperature process from $\left(\mathrm{T}_{1}, \mathrm{P}_{0}\right)$ to $\left(\mathrm{T}_{1}, \mathrm{P}_{1}\right)$ is defined as follows:

$$
s\left(T_{1}, P_{1}\right)-s\left(T_{1}, P_{0}\right)=\int_{P_{0}}^{P_{1}} d s=\int_{P_{0}}^{P_{1}}\left[\frac{\partial h /\left.\partial P\right|_{T}}{T_{1}}-\frac{1}{\rho\left(T_{1}, P\right) T_{1}}\right] d P
$$

These equations can be numerically integrated to calculate the total entropy as a function of temperature and pressure in order to create property data tables for rocket engine design codes.

\subsubsection{ENTHALPY, ENTROPY, AND DENSITY DATA TABLE CREATION}

Molecular dynamics runs of the HEDM compounds are performed using the updated intermolecular parameters to populate the data tables needed for rocket engine design codes. Molecular dynamics simulations are performed at appropriate temperature and pressure combinations for rocket engine analysis. The temperature and pressure combinations are chosen to cover the entire range of temperatures and pressures that the HEDM propellant will experience while flowing through the different engine components.

An example REDTOP-2 [64] propellant input file format is shown in Figure 3.19. For REDTOP-2, the top of the input file defines the molecule (number of atoms of each element and molecular weight). Also included are some data specification options, the number of pressures and temperatures included in the data file, and whether or not REDTOP-2 should calculate the gaseous density using the perfect gas equation. After this top part of the input file is defined, the rest of the input file is a table of thermophysical properties. The thermophysical properties calculated by molecular dynamics simulations are placed in this part of the REDTOP-2 propellant input file. 
Special attention must be paid to the units for each thermophysical property to avoid a unit conversion error.

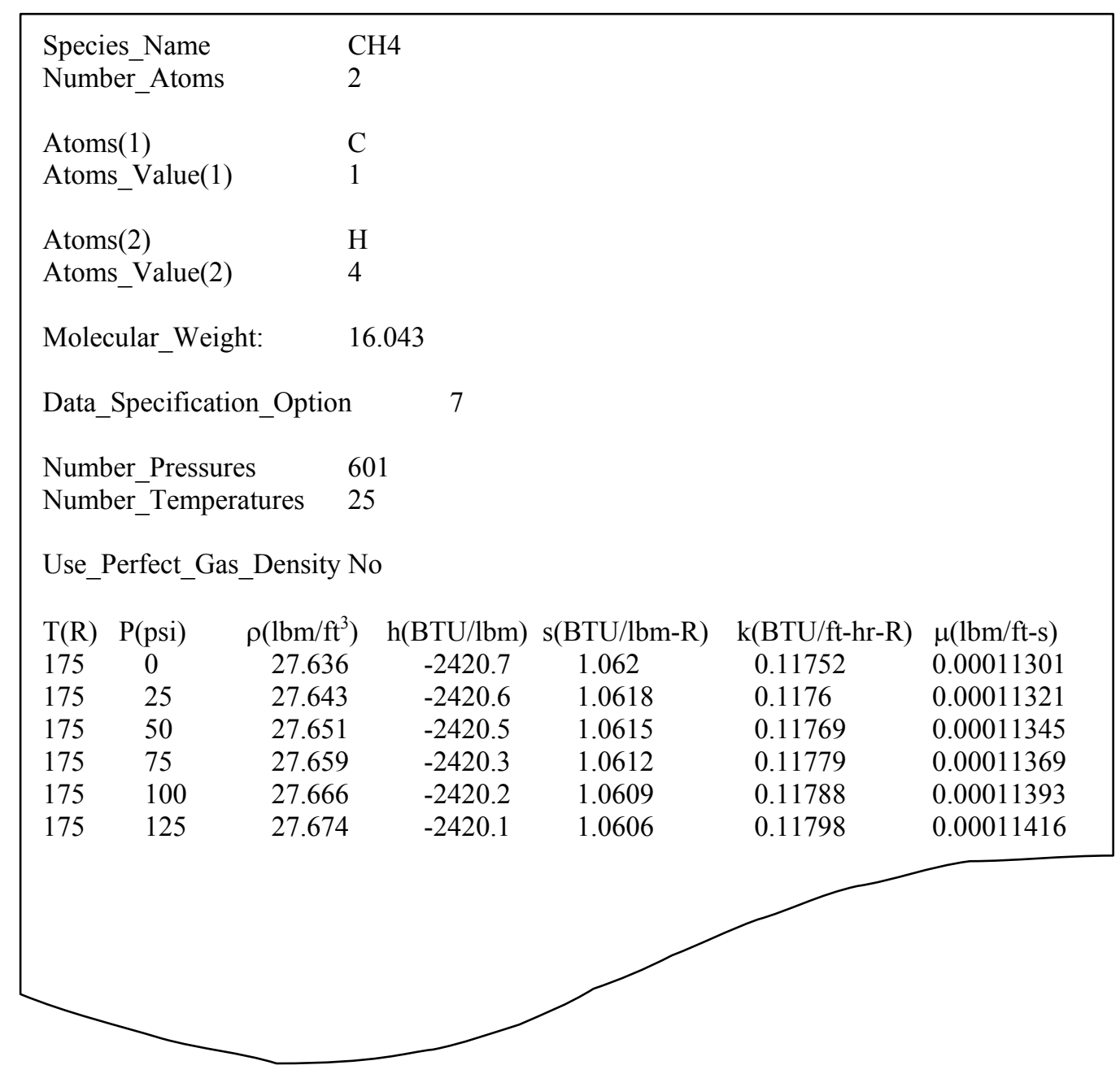

Figure 3.19: Sample REDTOP-2 Propellant Input File

\subsection{AdDitivity MethodS}

The third and final part of the thermophysical property calculation method is the use of additivity methods to determine the kinematic viscosity and thermal conductivity of the compounds of interest. Additivity methods are used instead of molecular dynamics for these two properties based upon the results of the sensitivity study discussed in Chapter 1. In this sensitivity study, it was shown that $\mathrm{I}_{\mathrm{sp}}$ is fairly insensitive to changes 
in kinematic viscosity and thermal conductivity. As a result, the predictions of kinematic viscosity and thermal conductivity do not need to be as accurate as the predictions of enthalpy, entropy, and density.

While not the only parameter affected by these properties, $I_{\mathrm{sp}}$ is the most important. Kinematic viscosity and thermal conductivity are important parameters in the calculation of pump efficiency and propellant feed line pressure losses [65]. These both contribute to the weight estimation of engine components. However, these effects are small compared to the effect of $I_{s p}$ when examining vehicle level metrics like gross vehicle weight.

As discussed in Chapter 2, additivity methods make use of the observation that a substance's physical properties depend on that substance's particular molecular structure [26,28, p.24]. The additivity methods used in this work were developed by Chung et al. [66]. Chung's method is a general multiparameter correlation that can be used to predict the viscosity and thermal conductivity of a wide range of hydrocarbons in the liquid, supercritical, and gaseous states and are based upon an input temperature and density [66]. Chung found the average absolute deviation for condensed phase shear viscosity to be $4 \%$ and for condensed phase thermal conductivity to be $8.6 \%$ for the hydrocarbons upon which Chung's correlations are based [66].

The temperature range in which the additivity methods are used in this work is the same temperature range used for the molecular dynamics simulations of the HEDM compound. The densities used as input into the additivity method model come from the computed densities from the molecular dynamics simulations at the desired temperature and pressure. 


\subsubsection{DESCRIPTION AND IMPLEMENTATION OF CHUNG'S METHOD}

Chung's additivity methods extend the additivity work performed by Chapman and Cowling [67] for dilute gas viscosity and thermal conductivity. Chung lists the following equations for the calculation of the dilute gas shear viscosity:

$$
\eta_{0}=\left(4.0785 \times 10^{-5}\right) \frac{(M T)^{0.5}}{V_{c}^{2 / 3} \Omega^{*}} F_{c}
$$

In equation (41), $\eta_{0}$ is the dilute gas shear viscosity in $\mathrm{P}, M$ is the molecular weight in $\mathrm{g} / \mathrm{mol}, T$ is the temperature in Kelvin, $V_{c}$ is the critical volume in $\mathrm{cm}^{3} / \mathrm{mol}, F_{C}$ and $\Omega^{*}$ are empirical coefficients defined with the following equations:

$$
\begin{gathered}
F_{c}=1-0.2756 \omega \\
\Omega^{*}=\frac{A}{T^{* B}}+\frac{C}{\exp \left(D T^{*}\right)}+\frac{E}{\exp \left(F T^{*}\right)}+G T^{* B} \sin \left(S T^{* W}-H\right)
\end{gathered}
$$

The empirical coefficients in the $\mathrm{W}^{*}$ equation are defined by Neufield et al. [68] and equal defined as: $\mathrm{A}=1.16145, \mathrm{~B}=0.14874, \mathrm{C}=0.52487, \mathrm{D}=0.77320, \mathrm{E}=2.16178$, $\mathrm{F}=2.43787, \mathrm{G}=-6.435^{*} 10^{-4}, \mathrm{H}=7.27371, \mathrm{~S}=18.0323$, and $\mathrm{W}=-0.76830$. In the above equations, $\omega$ is the acentric factor, $\mathrm{T}^{*}$ is the dimensionless temperature defined with the following equation:

$$
T^{*}=\frac{k_{B} T}{\varepsilon}
$$

where $k_{B}$ is Boltzmann's constant and $\varepsilon$ is the potential energy parameter, which is related to the critical temperature, $T_{c}$ (Kelvin), with the following equation: 


$$
\varepsilon=\frac{T_{c} k_{B}}{1.2593}
$$

The acentric factor, w, is defined by Pitzer [69] to equal:

$$
\omega=-1-\log _{10}\left(\frac{P_{\text {vap }}}{P_{c}}\right)
$$

where $P_{\text {vap }}$ is the vapor pressure at $\mathrm{T}=0.7^{*} \mathrm{~T}_{\mathrm{c}}$, the critical temperature (Kelvin) and $P_{c}$ is the critical pressure. The above list of equations is based upon the critical point of the substance of interest (critical density, temperature, and pressure). For this research work, the critical point is taken from literature values if available. If these values are not available in the literature, then the critical point is predicted using Joback's group contributions [70]. The equations used by Joback for computing the boiling temperature and the critical point are provided below:

$$
\begin{gathered}
T_{b}=198+\sum T_{b_{-} \text {contributions }} \\
T_{c}=\frac{T_{b}}{0.584+0.965 \sum T_{c_{-} \text {contributions }}-\left(\sum T_{c_{-} \text {contributions }}\right)^{2}} \\
P_{c}=\frac{1}{0.113+0.0032 n-\sum P_{c_{-} \text {contributions }}} \\
V_{c}=17.5+\sum V_{c_{-} \text {contributions }}
\end{gathered}
$$

In the above equations, $T_{b}$ is the boiling point temperature in $\mathrm{K}, T_{c}$ is the critical temperature in $\mathrm{K}, P_{c}$ is the critical pressure in bar, $V_{c}$ is the critical volume in liters $/ \mathrm{kmol}$, 
$n$ is the number of atoms in the molecule of interest, and the summations are all group contributions of different atoms and groups in the molecule of interest. Table 3.3 lists the group contributions developed by Joback [70].

Table 3.3: Joback Group Contributions [70]

\begin{tabular}{clcccc}
\hline & & $T_{C}$ & $P_{C}$ & $V_{c}$ & $T_{b}$ \\
\hline Nonring Increments & & & & & \\
& $-\mathrm{CH}_{3}$ & 0.0141 & -0.0012 & 65 & 23.58 \\
& $-\mathrm{CH}_{2^{-}}$ & 0.0189 & 0.0000 & 56 & 22.88 \\
& $>\mathrm{CH}-$ & 0.0164 & 0.0020 & 41 & 21.74 \\
& $>\mathrm{C}<$ & 0.0067 & 0.0043 & 27 & 18.25 \\
& $=\mathrm{CH}_{2}$ & 0.0113 & -0.0028 & 56 & 18.18 \\
& $=\mathrm{CH}-$ & 0.0129 & -0.0006 & 46 & 24.96 \\
& $=\mathrm{C}<$ & 0.0117 & 0.0011 & 38 & 24.14 \\
& $=\mathrm{C}=$ & 0.0026 & 0.0028 & 36 & 26.15 \\
& $\equiv \mathrm{CH}$ & 0.0027 & -0.0008 & 46 & 9.20 \\
& $\equiv \mathrm{C}-$ & 0.0020 & 0.0016 & 37 & 27.38 \\
Ring Increments & & & & \\
& $-\mathrm{CH} 2^{-}$ & 0.0100 & 0.0025 & 48 & 27.15 \\
& $>\mathrm{CH}-$ & 0.0122 & 0.0004 & 38 & 21.78 \\
& $>\mathrm{C}<$ & 0.0042 & 0.0061 & 27 & 21.32 \\
& $=\mathrm{CH}-$ & 0.0082 & 0.0011 & 41 & 26.73 \\
& $=\mathrm{C}<$ & 0.0143 & 0.0008 & 32 & 31.01 \\
Nitrogen Increments & & & & \\
& $-\mathrm{NH} \mathrm{H}_{2}$ & 0.0248 & 0.0109 & 36 & 73.23 \\
$>\mathrm{NH}$ (nonring) & 0.0295 & 0.0077 & 35 & 50.17 \\
$>\mathrm{NH}$ (ring) & 0.0130 & 0.0114 & 29 & 52.82 \\
$>\mathrm{N}-$ (nonring) & 0.0169 & 0.0074 & 9 & 11.74 \\
$-\mathrm{N}=$ (nonring) & 0.0255 & -0.0099 & 0 & 74.60 \\
& $-\mathrm{N}=$ (ring) & 0.0085 & 0.0076 & 34 & 57.55 \\
& $-\mathrm{CN}$ & 0.0496 & -0.0101 & 91 & 125.66 \\
& $-\mathrm{NO}{ }_{2}$ & 0.0437 & 0.0064 & 91 & 152.54 \\
\hline & & & & &
\end{tabular}

The dilute gas thermal conductivity is defined based upon the dilute gas shear viscosity using the following equations:

$$
\begin{gathered}
\lambda_{0}=7.452\left(\eta_{0} / M\right) \Psi \\
\Psi=1+\alpha \frac{0.215+0.28288 \alpha-1.061 \beta+0.26665 Z}{0.6366+\beta Z+1.061 \alpha \beta} \\
\alpha=\frac{c_{v}}{R}-3 / 2
\end{gathered}
$$




$$
\begin{gathered}
\beta=0.7862-0.7109 \omega+1.3168 \omega^{2} \\
Z=2.0+10.5\left(\frac{T}{T_{c}}\right)^{2}
\end{gathered}
$$

In the above equations, $\lambda_{0}$ is the dilute gas thermal conductivity in cal $/ \mathrm{cm}-\mathrm{s}-\mathrm{K}, c_{\nu}$ is the ideal gas heat capacity at constant volume in cal $/ \mathrm{mol}-\mathrm{K}$, and $R$ is the ideal gas constant (1.987 cal/mol-K).

The dilute gas shear viscosity and thermal conductivity are then used to compute the condensed phase shear viscosity and thermal conductivity [66]. The following relations are used to calculate the condensed phase shear viscosity:

$$
\begin{gathered}
\eta=\eta_{k}+\eta_{p} \\
\eta_{k}=\eta_{0}\left(1 / G_{2}+A_{6} Y\right) \\
\eta_{p}=\left[\frac{3.6344 \times 10^{-5}\left(M T_{c}\right)^{0.5}}{V_{c}^{2 / 3}}\right] A_{7} Y^{2} G_{2} \exp \left(A_{8}+A_{9} / T^{*}+A_{10} / T^{* 2}\right) \\
Y=\frac{\rho V_{c}}{6} \\
G_{1}=\frac{1.0-0.5 Y}{(1-Y)^{3}} \\
G_{2}=\frac{A_{1}\left[1-\exp \left(-A_{4} Y\right)\right] / Y+A_{2} G_{1} \exp \left(A_{5} Y\right)+A_{3} G_{1}}{A_{1} A_{4}+A_{2}+A_{3}}
\end{gathered}
$$

In the above equations, $\eta_{0}$ is the dilute gas viscosity. The constants $A_{1}$ through $A_{10}$ are defined with the following equation and Table 3.4 below: 


$$
A_{i}=a_{0}(i)+a_{1}(i) \omega
$$

Table 3.4: Constants Used for Condensed Phase Shear Viscosity Relation [66]

\begin{tabular}{ccc}
\hline$i$ & $a_{0}(i)$ & $a_{1}(i)$ \\
\hline 1 & 6.32402 & 50.4119 \\
2 & $1.21 \mathrm{E}-03$ & $-1.15 \mathrm{E}-03$ \\
3 & 5.28346 & 254.209 \\
4 & 6.62263 & 38.0957 \\
5 & 19.7454 & 7.63034 \\
6 & -1.89992 & -12.5367 \\
7 & 24.2745 & 3.44945 \\
8 & 0.79716 & 1.11764 \\
9 & -0.23816 & $6.77 \mathrm{E}-02$ \\
10 & $6.86 \mathrm{E}-02$ & 0.34793 \\
\hline
\end{tabular}

The condensed phase thermal conductivity is defined in a similar manner as the condensed phase shear viscosity. The following equations are used to define the condensed phase thermal conductivity:

$$
\begin{gathered}
\lambda=\lambda_{k}+\lambda_{p} \\
\lambda_{k}=\lambda_{0}\left(1 / H_{2}+B_{6} Y\right) \\
\lambda_{p}=\left[\frac{3.039 \times 10^{-4}\left(T_{c} / M\right)^{0.5}}{V_{c}^{2 / 3}}\right] B_{7} Y^{2} H_{2}\left(\frac{T}{T_{c}}\right)^{0.5} \\
H_{2}=\frac{B_{1}\left[1-\exp \left(-B_{4} Y\right)\right] / Y+B_{2} G_{1} \exp \left(B_{5} Y\right)+B_{3} G_{1}}{B_{1} B_{4}+B_{2}+B_{3}}
\end{gathered}
$$

In the above equations, $\lambda_{0}$ is the dilute gas thermal conductivity. The constants $B_{1}$ through $B_{7}$ are defined with the following equation and Table 3.5 below:

$$
B_{i}=b_{0}(i)+b_{1}(i) \omega
$$


Table 3.5: Constants Used for Condensed Phase Thermal Conductivity Relation [66]

\begin{tabular}{ccc}
\hline$i$ & $b_{0}(i)$ & $b_{1}(i)$ \\
\hline 1 & 2.41657 & 0.74824 \\
2 & -0.50924 & -1.50936 \\
3 & 6.61069 & 5.62073 \\
4 & 14.5425 & -8.91387 \\
5 & 0.79274 & 0.82019 \\
6 & -5.8634 & 12.8005 \\
7 & 81.171 & 114.158 \\
\hline
\end{tabular}

\subsubsection{CREATING Viscosity AND Thermal ConduCtivity DAta Tables}

With all the relations established for computing both the dilute and condensed phase shear viscosity and thermal conductivity, data tables can be created and imported into the appropriate rocket engine powerhead design code. The same temperature and pressure ranges used for the enthalpy, entropy, and density calculations (the ranges that one expects the propellant to experience while flowing through the rocket engine) are used for shear viscosity and thermal conductivity.

In the REDTOP-2 input file example shown in Figure 3.19, the two columns in the bottom right of the figure are the thermal conductivity and shear viscosity. As with importing enthalpy, entropy, and density, care must be taken to ensure that the correct units are used in any propellant input file.

An additional step in the additivity method process presented in this research work is needed to initially validate that this additivity method process can be used for the two HEDM compounds of interest: DMAZ and quadricyclane. The additivity method predictions are compared with experimentally measured values to ensure that the predictions are sufficiently accurate. The level of accuracy required is determined by the engine level sensitivity study provided in Section 1.2. In this sensitivity study, it was found that a factor of five increase or decrease in kinematic viscosity resulted in a 0.13 second change in vacuum $I_{s p}$ and a factor of five increase or decrease in thermal conductivity resulted in a 0.14 second change in vacuum $I_{s p}$. These vacuum $I_{s p}$ 
sensitivities are for a staged-combustion cycle rocket engine. It is expected that vacuum $I_{s p}$ is more sensitive to changes in kinematic viscosity and thermal conductivity for expander cycle engines, but not a great deal more.

As a result, in order to keep the error in vacuum $I_{s p}$ due to errors in kinematic viscosity and thermal conductivity under 0.25 seconds (an acceptable error in predicted vacuum $I_{s p}$ for conceptual vehicle design), a conservative error of $150 \%$ is deemed acceptable in the additivity method predictions of kinematic viscosity and thermal conductivity.

\subsection{EXPERIMENTAL METHODS}

In general application of the method developed in this thesis work, little or no experimental testing is needed. This is because the method uses previously published experimental data from model compounds to determine the appropriate settings for the intermolecular potential parameters.

For this thesis research, however, validation of the computational results from the quantum mechanical, molecular dynamics, and group additivity method analyses is necessary. This validation is done by comparing the computational results with experimentally measured thermophysical properties. Experimental data for quadricyclane was previously published by Wucherer et al. [1]. However, very little published experimental data exists for the other HEDM compound of interest, DMAZ. As a result, experimental testing of DMAZ was performed as part of this thesis work. A sample of DMAZ was obtained from MACH I, Inc. [71], a 3M distributor. The sample had a quoted purity of $99.5 \%$ DMAZ.

Experimental data at relatively low temperatures $(\mathrm{T}<450 \mathrm{~K})$ and pressures $(\mathrm{P}<20$ atm) was obtained in laboratories in the School of Chemical and Biomolecular Engineering and in the School of Materials Science and Engineering at Georgia Tech. Laboratory measurements of density, kinematic viscosity, and thermal conductivity of 
DMAZ were performed with the help of Dr. Tongfan Sun and Professor Amyn Teja in the School of Chemical and Biomolecular Engineering at Georgia Tech. Laboratory measurements of specific heat were performed with the help of Lex Nunnery, Jeremy Walker, and Professor Naresh Thadhani in the School of Materials Science and Engineering at Georgia Tech. ASTM standard testing procedures were followed in all cases except for the measurement of thermal conductivity. Thermal conductivity was measured using a testing procedure developed by Bleazard and Teja [72].

\subsubsection{DENSITY}

Density was measured using a high pressure pycnometer such as the one shown in Figure 3.20. The ASTM standard D 1481-02 [73] was followed to obtain density measurements. A detailed description of the procedure used to measure density can be found in this ASTM standard. An overview of this procedure is provided here:

1. Measure the weight of a known volume of the sample fluid in the pycnometer (the pycnometer has a very fine line cut with a diamond pencil below which the volume is precisely known).

2. Calculate density of sample fluid with measured weight and known volume.

3. Repeat for each temperature - the temperature is set by submersing the pycnometer into a bath of liquid water at a controlled temperature and allowing the pycnometer to equilibrate its temperature with the bath.

Table 3.6: Measured Density of DMAZ $(\rho)$

\begin{tabular}{ccc}
\hline$P(\mathrm{~atm})$ & $T(\mathrm{~K})$ & $\rho\left(\mathrm{kg} / \mathrm{m}^{3}\right)$ \\
\hline 1.00 & 293.25 & 933.5 \\
1.00 & 313.00 & 913.5 \\
1.00 & 333.10 & 892.6 \\
1.00 & 353.05 & 872.1 \\
1.00 & 368.05 & 857.5 \\
1.00 & 387.35 & 836.4 \\
8.00 & 404.55 & 816.5 \\
11.00 & 418.55 & 796.2 \\
19.00 & 435.95 & 776.6 \\
\hline
\end{tabular}




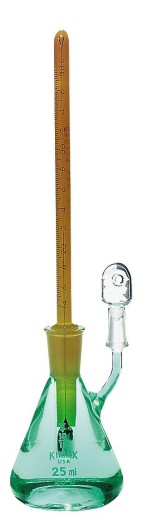

Figure 3.20: Cole-Farmer Pycnometer

\subsubsection{VISCOSITY}

The shear viscosity was measured using a high pressure Cannon-Fenske viscometer such as the one shown in Figure 3.21. The ASTM standards D 445-04 [74] and D 446-04 [75] were followed to obtain viscosity measurements. A detailed description of the procedure used to measure viscosity can be found in these ASTM standards. An overview of this procedure is provided here:

1. Fill the viscometer with the sample fluid.

2. Draw the sample fluid through the working capillary and bulb (location $\mathrm{C}$ in Figure 3.21) so that the lower level of the fluid is above the timing mark (location E), place rubber stoppers into the tubes to hold the test portion in place.

3. Insert the viscometer in the constant temperature bath.

4. Allow the viscometer to reach the bath temperature and remove the stoppers.

5. Measure how long the lower level of fluid takes to run from location $\mathrm{E}$ to location F.

6. Calculate the kinematic viscosity based upon this measured time and the type of viscometer used. 
Table 3.7: Measured Shear Viscosity of DMAZ $(\mu)$

\begin{tabular}{ccc}
\hline$P(\mathrm{~atm})$ & $T(\mathrm{~K})$ & $\mu(\mathrm{cP})$ \\
\hline 1.00 & 293.25 & 0.721 \\
1.00 & 313.00 & 0.564 \\
1.00 & 333.10 & 0.456 \\
1.00 & 353.05 & 0.379 \\
1.00 & 368.05 & 0.335 \\
1.00 & 387.35 & 0.284 \\
1.00 & 406.05 & 0.255 \\
8.00 & 406.35 & 0.249 \\
15.00 & 421.55 & 0.235 \\
19.00 & 438.95 & 0.209 \\
\hline
\end{tabular}

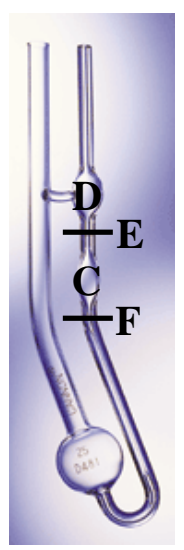

Figure 3.21: Cannon-Fenske Routine Viscometer

\subsubsection{THERMAL CONDUCTIVITY}

The thermal conductivity was measured using a relative transient hot-wire method with a Pyrex capillary filled with mercury (see Figure 3.22). A detailed description of the procedure used to measure thermal conductivity can be found in reference 72 . An overview of this procedure is provided here:

1. Fill the hot-wire cell with sample fluid.

2. Close the control relay to apply a step voltage to the bridge.

3. The wire in the hot-wire cell is subjected to this voltage and allowed to heat. This results in an increase in its resistance. 
4. Adjust the decade resistor to balance the bridge and calculate the rise in the resistance.

5. The thermal conductivity of the sample fluid is determined from this rise in resistance.

Table 3.8: Measured Thermal Conductivity of DMAZ

\begin{tabular}{ccc}
\hline$P(\mathrm{~atm})$ & $T(\mathrm{~K})$ & $\lambda(\mathrm{W} / \mathrm{m}-\mathrm{K})$ \\
\hline 1.00 & 297.40 & 0.133 \\
1.00 & 324.90 & 0.125 \\
1.00 & 349.30 & 0.118 \\
8.00 & 374.30 & 0.112 \\
11.00 & 398.50 & 0.107 \\
19.00 & 425.30 & 0.101 \\
\hline
\end{tabular}

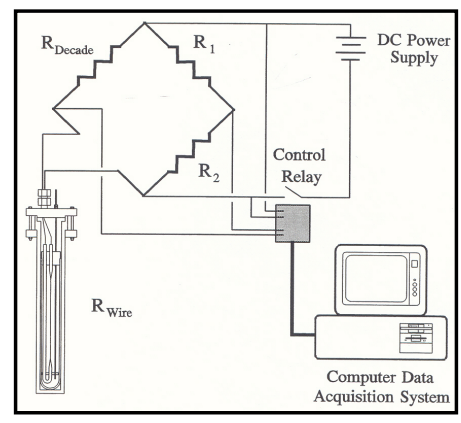

Figure 3.22: Transient Hot-Wire Cell

\subsubsection{SPECIFIC HEAT}

The specific heat was measured using a TA Instruments differential scanning calorimeter (DSC) such as the one shown in Figure 3.23. The samples were weighed using a TA Instruments thermogravimetric analyzer (TGA) such as the one shown in Figure 3.24. The ASTM standard E 1269-04 [76] was followed to obtain specific heat measurements. A detailed description of the procedure used to measure thermal 
conductivity can be found in this ASTM standard. An overview of this procedure is provided here:

1. Weigh a specimen holder using TGA.

2. Fill the specimen holder with a sample fluid.

3. Use a crimper to seal sample fluid.

4. Weigh specimen holder and sample fluid in TGA to measure weight of sample fluid.

5. Place specimen holder with sample fluid into DSC and set DSC to desired starting temperature for test.

6. Ramp up temperature and record the thermal response.

7. The specific heat of the sample fluid can be calculated from the recorded thermal response and measured weight.

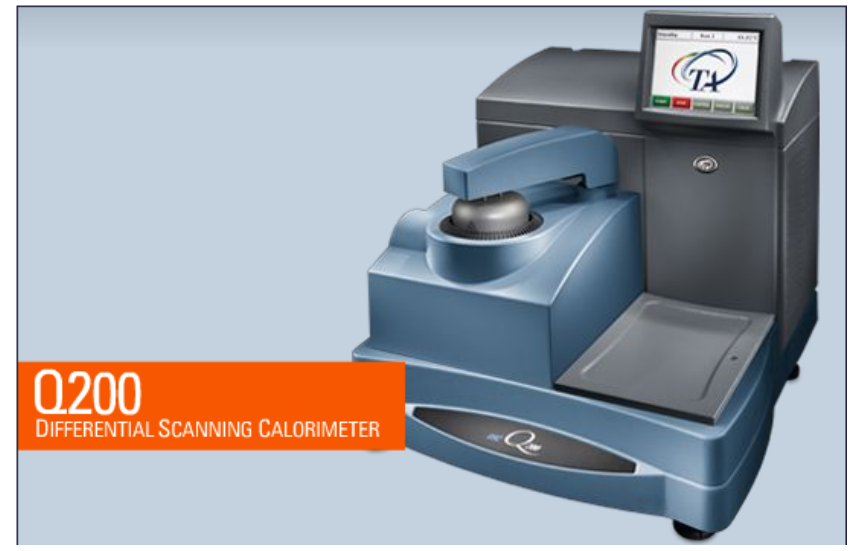

Figure 3.23: TA Instruments Differential Scanning Calorimeter 


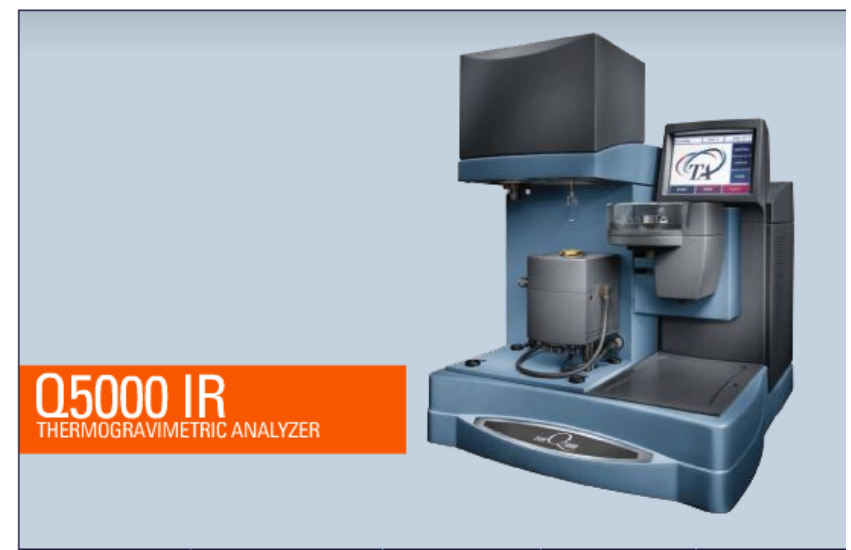

Figure 3.24: TA Instruments Thermogravimetric Analyzer

Table 3.9: Measured Specific Heat of DMAZ $\left(\mathrm{C}_{\mathrm{P}}\right)$

\begin{tabular}{ccc}
\hline$P(\mathrm{~atm})$ & $T(\mathrm{~K})$ & $C_{P}(\mathrm{~J} / \mathrm{g}-\mathrm{K})$ \\
\hline 1.00 & 320 & 1.782 \\
1.00 & 325 & 1.812 \\
1.00 & 330 & 1.817 \\
1.00 & 335 & 1.819 \\
1.00 & 340 & 1.820 \\
1.00 & 345 & 1.823 \\
1.00 & 350 & 1.823 \\
1.00 & 355 & 1.825 \\
1.00 & 360 & 1.825 \\
1.00 & 365 & 1.825 \\
1.00 & 370 & 1.830 \\
1.00 & 375 & 1.850 \\
\hline
\end{tabular}




\section{CHAPTER 4}

\section{FINAL APPLICATION}

An application of the thermophysical property calculation method described in this work was performed for two different HEDM compounds: Quadricyclane and DMAZ. A model compound is identified for each HEDM compound and utilized in the thermophysical property calculation method to determine the appropriate values for the intermolecular parameters of the COMPASS model to be used for the corresponding HEDM compound. This is done due to the fact that there is generally limited experimental data for HEDM compounds, but more extensive data for their corresponding model compounds. Model compounds are chosen based upon their having a similar molecular structure to their corresponding HEDM compounds.

\subsection{Model CoMpounds: NorbornANe AND Ethyl AZIDE}

The first step in calculating the thermophysical properties of DMAZ and quadricyclane is to select a model compound for each HEDM compound. The rules defined in Section 3.3 are used to select the appropriate model compounds. These rules are repeated here:

\section{Rule 1: The model compound must have a "similar" molecular structure as the corresponding HEDM compound.}

For DMAZ, this rule means that the model compound must have an azide chain (near linear chain of three nitrogen atoms). For quadricyclane, this rule means that the model compound must be a bicyclic compound (a molecule with two fused rings). 


\section{Rule 2: $\quad$ The model compound must have the same atom types as the HEDM compound.}

For DMAZ, this means that the molecule must have the three nitrogen atom types in the azide chain, a carbon atom type attached to the end of the azide chain, and a nonpolar hydrogen atom type. For quadricyclane, this means that the molecule must have two carbon atom types: one with two heavy atoms attached and one with three heavy atoms attached. The molecule must also have a nonpolar hydrogen atom type.

\section{Rule 3: The model compound must have some density and enthalpy (or specific heat) experimental data in the liquid phase.}

The model compound that meets the requirements of the first two rules must also have some density and enthalpy or specific heat experimental data. Extensive experimental data is preferable, but will often times not exist due to the potentially strict requirements set forth in rules 1 and 2 .

Using these three rules, the model compounds chosen for DMAZ and quadricyclane are ethyl azide and norbornane respectively. Figure 4.1 provides ball and cylinder renderings of ethyl azide and DMAZ. As can be seen from the ball and cylinder renderings, ethyl azide has the same azide chain found in DMAZ. Ethyl azide also has the same three nitrogen atom types and the same carbon atom type attached to the end of the azide chain. Ethyl azide also has a nonpolar hydrogen atom type like DMAZ. The standard carbons and nitrogen found on the right side of the DMAZ molecule in Figure 4.1 were not required in the model compound, because their intermolecular parameters are well defined in the COMPASS model (the baseline COMPASS values for these atom types were used in the modeling of DMAZ) and they do not play a significant role in the 
prediction of density. The outer atoms of the molecule are most important in the prediction of the liquid density of a substance [59].

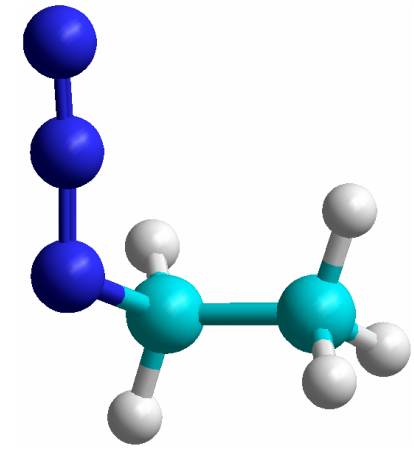

(a)

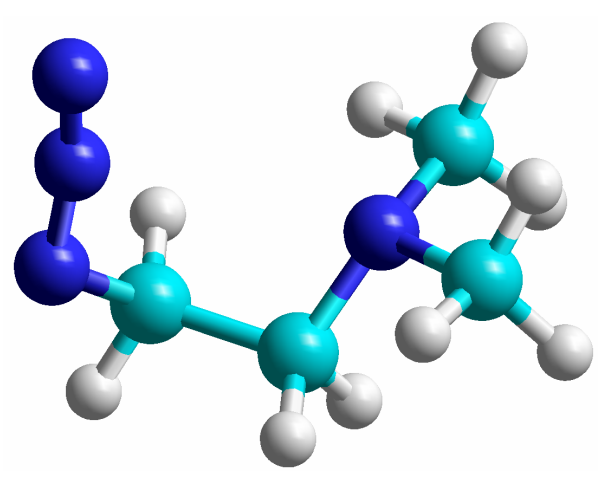

(b)

Figure 4.1: Ball and Cylinder Renderings of (a) Ethyl Azide and (b) DMAZ

Figure 4.2 provides ball and cylinder renderings for norbornane and quadricyclane. Norbornane is a bicyclic compound with the top three carbon atoms in Figure 4.2a belonging to two different five-carbon rings. Like quadricyclane, norbornane has two different carbon atom types: a carbon attached to two heavy atoms (the top carbon atom and bottom four carbon atoms in Figure 4.2a are this atom type) and a carbon atom attached to three heavy atoms (the middle two carbon atoms are this atom type). Norbornane also has the same nonpolar hydrogen atom type as quadricyclane.

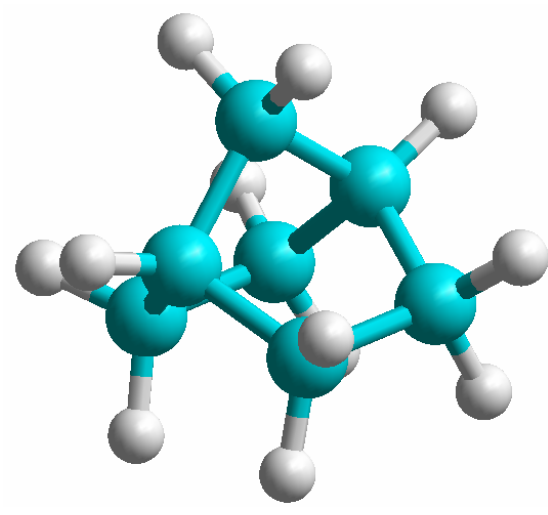

(a)

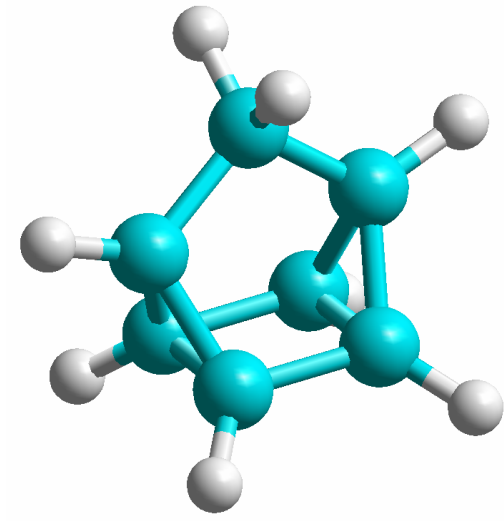

(b)

Figure 4.2: Ball and Cylinder Renderings of (a) Norbornane and (b) Quadricyclane 


\subsubsection{QUANTUM MECHANICS}

With the model compounds ethyl azide and norbornane chosen, quantum mechanical techniques are performed for two main purposes:

1. Find the minimum energy configuration of each model compound in order to perform vibrational normal mode quantum mechanical analyses and molecular dynamics simulations.

2. Calculate the normal mode vibrational frequencies in order to calculate the ideal gas sensible enthalpy.

\section{Determination of Minimum Energy Configuration}

The minimum energy configuration of each model compound is determined using the GAMESS quantum mechanical software. An initial molecular configuration (initial guess) is needed as input to GAMESS to start the optimization process. These initial molecular configurations are provided to GAMESS from a molecular dynamics conformational analysis performed using the LAMMPS molecular dynamics software [38]. Table 4.1 and Table 4.2 provide the results of the LAMMPS conformational search and GAMESS optimization for norbornane and ethyl azide respectively. As can be seen in Table 4.1, the LAMMPS conformational search provides a very good starting point for the norbornane geometry and only a small refinement by GAMESS is required. For ethyl azide (Table 4.2), the LAMMPS conformational provides a poorer starting point that requires more refinement from the GAMESS optimizer.

The minimum energy configurations found by GAMESS are used for quantum mechanical vibrational analyses and for molecular dynamics simulations. As discussed in section 3.2.1, a DFT energy calculation method with the B3LYP functional and the 6$31 \mathrm{G}^{*}$ basis set is used for all GAMESS energy calculations. 
Table 4.1: Atomic Coordinates for Optimized Norbornane Geometry

\begin{tabular}{ccccccc}
\hline & Conformational Search Results & \multicolumn{3}{c}{ GAMESS Results } \\
\hline Atom & $X(\AA)$ & $Y(\AA)$ & $Z(\AA)$ & $X(\AA)$ & $Y(\AA)$ & $Z(\AA)$ \\
\hline C & -0.194 & 0.078 & 2.109 & -0.192 & 0.079 & 2.097 \\
C & -0.064 & -1.061 & 1.065 & -0.063 & -1.058 & 1.059 \\
C & 0.081 & 1.208 & 1.083 & 0.084 & 1.205 & 1.075 \\
C & -1.094 & -0.619 & 0.006 & -1.109 & -0.630 & 0.001 \\
C & 1.336 & -0.780 & 0.486 & 1.351 & -0.794 & 0.487 \\
C & 1.433 & 0.758 & 0.492 & 1.450 & 0.769 & 0.493 \\
C & -0.998 & 0.919 & 0.020 & -1.012 & 0.933 & 0.016 \\
H & 0.575 & 0.023 & 2.909 & 0.558 & 0.025 & 2.897 \\
H & -1.206 & 0.140 & 2.561 & -1.188 & 0.143 & 2.552 \\
H & -0.208 & -2.090 & 1.444 & -0.205 & -2.076 & 1.437 \\
H & 0.073 & 2.241 & 1.479 & 0.075 & 2.227 & 1.468 \\
H & -2.119 & -0.958 & 0.290 & -2.113 & -0.968 & 0.285 \\
H & -1.971 & 1.378 & 0.316 & -1.965 & 1.382 & 0.319 \\
H & -0.852 & -1.037 & -0.999 & -0.893 & -1.057 & -0.986 \\
H & -0.708 & 1.323 & -0.977 & -0.752 & 1.351 & -0.963 \\
H & 1.445 & -1.195 & -0.542 & 1.475 & -1.220 & -0.515 \\
H & 2.125 & -1.232 & 1.134 & 2.121 & -1.240 & 1.128 \\
H & 2.277 & 1.104 & 1.136 & 2.272 & 1.110 & 1.133 \\
H & 1.585 & 1.164 & -0.535 & 1.622 & 1.184 & -0.506 \\
\hline
\end{tabular}

Table 4.2: Atomic Coordinates for Optimized Ethyl Azide Geometry

\begin{tabular}{ccccccc}
\hline & Conformational Search Results & \multicolumn{3}{c}{ GAMESS Results } \\
\hline Atom & $X(\AA)$ & $Y(\AA)$ & $Z(\AA)$ & $X(\AA)$ & $Y(\AA)$ & $Z(\AA)$ \\
\hline $\mathrm{N}$ & -1.603 & 0.630 & -3.827 & -1.229 & 0.511 & -3.933 \\
$\mathrm{~N}$ & -1.258 & -0.208 & -3.156 & -1.177 & -0.275 & -3.160 \\
$\mathrm{~N}$ & -1.092 & -1.231 & -2.436 & -1.215 & -1.160 & -2.311 \\
$\mathrm{C}$ & 0.199 & -1.558 & -1.811 & 0.112 & -1.540 & -1.797 \\
$\mathrm{C}$ & 0.697 & -0.530 & -0.793 & 0.661 & -0.541 & -0.785 \\
$\mathrm{H}$ & 0.987 & -1.753 & -2.577 & 0.813 & -1.678 & -2.616 \\
$\mathrm{H}$ & -0.027 & -2.518 & -1.313 & -0.035 & -2.503 & -1.331 \\
$\mathrm{H}$ & 1.441 & -1.014 & -0.123 & 1.610 & -0.889 & -0.389 \\
$\mathrm{H}$ & -0.127 & -0.184 & -0.136 & -0.031 & -0.416 & 0.039 \\
$\mathrm{H}$ & 1.117 & 0.307 & -1.35 & 0.826 & 0.432 & -1.240 \\
\hline
\end{tabular}

\section{Calculation of Ideal Gas Sensible Enthalpy}

With the minimum energy configurations of norbornane and ethyl azide determined, a normal mode vibrational analysis can be performed using GAMESS in order to calculate the normal mode vibrational frequencies. These vibrational frequencies 
are needed in order to calculate the ideal gas component of sensible enthalpy. Table 4.3 and Table 4.4 provide the results of the GAMESS normal mode vibrational analysis of norbornane and ethyl azide respectively. There are $3 \mathrm{~N}-6$ vibrational modes in a nonlinear molecule where $\mathrm{N}$ is the number of atoms in the molecule. For norbornane this translates into 51 vibrational modes $(3 * 19-6=51)$, and for ethyl azide this translates into 24 vibrational modes $(3 * 10-6=24)$. The 6 degrees of freedom subtracted from the $3 \mathrm{~N}$ are the 3 translational and 3 rotational ( 2 for a linear molecule) degrees of freedom. The wave number (second column in both Table 4.3 and Table 4.4) is the value calculated and outputted by GAMESS. The wave number is related to the vibrational frequency and characteristic vibrational temperature with the following equations:

$$
\begin{gathered}
v=c k \\
\theta_{v i b}=\frac{h v}{k_{B}}
\end{gathered}
$$

In the above equations, $v$ is the vibrational frequency, $c$ is the speed of light in a vacuum, $k$ is the wave number, $\theta_{\text {vib }}$ is the characteristic vibrational temperature, $h$ is Planck's constant, and $k_{B}$ is Boltzmann's constant. The vibrational contribution to the specific heat at constant volume from a particular vibrational mode is calculated with the following equation:

$$
C_{V}^{v i b}(T)=R\left(\frac{\theta_{v i b}}{T}\right)^{2} \frac{e^{\theta_{v i b} / T}}{\left(e^{\theta_{v i b} / T}-1\right)^{2}}
$$

where $R$ is the universal gas constant and $T$ is the temperature. 
Table 4.3: Normal Mode Vibrational Frequencies of Norbornane

\begin{tabular}{|c|c|c|c|}
\hline Mode & Wave \#, $k(1 / \mathrm{cm})$ & $\theta_{\text {vib }}(K)$ & $C_{V}^{v i b}-298.15 \mathrm{~K}(\mathrm{~J} / \mathrm{mol}-\mathrm{K})$ \\
\hline 1 & 151 & 217 & 7.9562 \\
\hline 2 & 330 & 475 & 6.7600 \\
\hline 3 & 400 & 576 & 6.1473 \\
\hline 4 & 461 & 663 & 5.5927 \\
\hline 5 & 546 & 786 & 4.8051 \\
\hline 6 & 761 & 1095 & 3.0019 \\
\hline 7 & 764 & 1100 & 2.9752 \\
\hline 8 & 810 & 1165 & 2.6540 \\
\hline 9 & 822 & 1183 & 2.5718 \\
\hline 10 & 825 & 1187 & 2.5543 \\
\hline 11 & 887 & 1277 & 2.1647 \\
\hline 12 & 899 & 1294 & 2.0951 \\
\hline 13 & 930 & 1338 & 1.9244 \\
\hline 14 & 959 & 1380 & 1.7759 \\
\hline 15 & 967 & 1391 & 1.7356 \\
\hline 16 & 971 & 1397 & 1.7151 \\
\hline 17 & 975 & 1403 & 1.6941 \\
\hline 18 & 1008 & 1450 & 1.5417 \\
\hline 19 & 1048 & 1508 & 1.3704 \\
\hline 20 & 1102 & 1586 & 1.1639 \\
\hline 21 & 1135 & 1633 & 1.0521 \\
\hline 22 & 1148 & 1652 & 1.0103 \\
\hline 23 & 1173 & 1688 & 0.9333 \\
\hline 24 & 1195 & 1720 & 0.8696 \\
\hline 25 & 1245 & 1791 & 0.7415 \\
\hline 26 & 1248 & 1796 & 0.7338 \\
\hline 27 & 1282 & 1845 & 0.6564 \\
\hline 28 & 1294 & 1862 & 0.6322 \\
\hline 29 & 1302 & 1873 & 0.6163 \\
\hline 30 & 1316 & 1894 & 0.5867 \\
\hline 31 & 1341 & 1929 & 0.5403 \\
\hline 32 & 1354 & 1948 & 0.5173 \\
\hline 33 & 1363 & 1960 & 0.5026 \\
\hline 34 & 1367 & 1967 & 0.4953 \\
\hline 35 & 1508 & 2169 & 0.3051 \\
\hline 36 & 1522 & 2189 & 0.2905 \\
\hline 37 & 1528 & 2199 & 0.2839 \\
\hline 38 & 1530 & 2202 & 0.2818 \\
\hline 39 & 1556 & 2239 & 0.2571 \\
\hline 40 & 3041 & 4376 & 0.0008 \\
\hline 41 & 3047 & 4384 & 0.0007 \\
\hline 42 & 3054 & 4394 & 0.0007 \\
\hline 43 & 3057 & 4398 & 0.0007 \\
\hline 44 & 3068 & 4415 & 0.0007 \\
\hline 45 & 3081 & 4432 & 0.0006 \\
\hline 46 & 3086 & 4440 & 0.0006 \\
\hline 47 & 3092 & 4448 & 0.0006 \\
\hline 48 & 3096 & 4455 & 0.0006 \\
\hline 49 & 3105 & 4468 & 0.0006 \\
\hline 50 & 3110 & 4475 & 0.0006 \\
\hline 51 & 3113 & 4480 & 0.0006 \\
\hline
\end{tabular}


Table 4.4: Normal Mode Vibrational Frequencies of Ethyl Azide

\begin{tabular}{cccc}
\hline Mode & Wave \#, $k(1 / \mathrm{cm})$ & $\theta_{\text {vib }}(\mathrm{K})$ & $C_{V}^{\text {vib }}-298.15 \mathrm{~K}(\mathrm{~J} / \mathrm{mol}-\mathrm{K})$ \\
\hline 1 & 67 & 96 & 8.2430 \\
2 & 196 & 283 & 7.7191 \\
3 & 287 & 414 & 7.1004 \\
4 & 400 & 576 & 6.1476 \\
5 & 574 & 826 & 4.5501 \\
\hline 6 & 662 & 952 & 3.7822 \\
7 & 809 & 1165 & 2.6576 \\
8 & 855 & 1230 & 2.3632 \\
9 & 1003 & 1442 & 1.5666 \\
10 & 1105 & 1591 & 1.1519 \\
11 & 1168 & 1681 & 0.9472 \\
12 & 1300 & 1870 & 0.6196 \\
13 & 1342 & 1931 & 0.5385 \\
14 & 1397 & 2011 & 0.4466 \\
15 & 1441 & 2073 & 0.3850 \\
16 & 1518 & 2184 & 0.2943 \\
17 & 1522 & 2189 & 0.2904 \\
18 & 1534 & 2207 & 0.2782 \\
19 & 2252 & 3240 & 0.0187 \\
20 & 3038 & 4371 & 0.0008 \\
21 & 3040 & 4374 & 0.0008 \\
22 & 3102 & 4463 & 0.0006 \\
23 & 3122 & 4491 & 0.0005 \\
24 & 3143 & 4522 & 0.0005 \\
\hline
\end{tabular}

The total specific heat at constant volume of a molecule is the sum of each of these vibrational contributions, the translational contribution, and the rotational contribution. This equation for a non-linear molecule is provided below:

$$
C_{V}^{i g}(T)=C_{P}^{i g}(T)-R=C_{V}^{t r}+C_{V}^{r o t}+C_{V}^{v i b}=\frac{3}{2} R+\frac{3}{2} R+\sum_{i=1}^{3 N-6} R\left(\frac{\theta_{v i b_{i}}}{T}\right)^{2} \frac{e^{\theta_{v i b_{i}} / T}}{\left(e^{\theta_{v b_{i}} / T}-1\right)^{2}}
$$

Table 4.5 and Table 4.6 provide the results of this calculation of the ideal gas specific heat and sensible enthalpy as a function of temperature for norbornane and ethyl azide respectively. The equation used to calculate the ideal gas sensible enthalpy (less RT) is provided below:

$$
h_{\text {sens }}^{i g}-R T=\int_{298.15 K}^{T} C_{V}^{i g}(\tau) d \tau
$$


Table 4.5: Ideal Gas Sensible Enthalpy Results for Norbornane

\begin{tabular}{cccccc}
\hline $\begin{array}{c}\text { Temperature } \\
(K)\end{array}$ & $\begin{array}{c}C_{V_{-} \text {trans }} \\
(\mathrm{J} / \mathrm{mol}-\mathrm{K})\end{array}$ & $\begin{array}{c}C_{V_{-} \text {rot }} \\
(\mathrm{J} / \mathrm{mol}-\mathrm{K})\end{array}$ & $\begin{array}{c}C_{V_{-} \text {vib }} \\
(\mathrm{J} / \mathrm{mol}-\mathrm{K})\end{array}$ & $\begin{array}{c}C_{V} \\
(\mathrm{~J} / \mathrm{mol}-\mathrm{K})\end{array}$ & $\begin{array}{c}h_{\text {sens }}^{\text {ig }}-R T \\
(\mathrm{~kJ} / \mathrm{mol})\end{array}$ \\
\hline 298.15 & 12.4715 & 12.4715 & 73.5130 & 98.4563 & 0.0000 \\
360.00 & 12.4715 & 12.4715 & 102.7500 & 127.6934 & 6.9937 \\
365.00 & 12.4715 & 12.4715 & 105.1030 & 130.0463 & 7.6380 \\
370.00 & 12.4715 & 12.4715 & 107.4480 & 132.3913 & 8.2941 \\
375.00 & 12.4715 & 12.4715 & 109.7840 & 134.7274 & 8.9619 \\
380.00 & 12.4715 & 12.4715 & 112.1110 & 137.0535 & 9.6414 \\
\hline
\end{tabular}

Table 4.6: Ideal Gas Sensible Enthalpy Results for Ethyl Azide

\begin{tabular}{cccccc}
\hline $\begin{array}{c}\text { Temperature } \\
(K)\end{array}$ & $\begin{array}{c}C_{V_{-} \text {trans }} \\
(\mathrm{J} / \mathrm{mol}-\mathrm{K})\end{array}$ & $\begin{array}{c}C_{V_{-} \text {rot }} \\
(\mathrm{J} / \mathrm{mol}-\mathrm{K})\end{array}$ & $\begin{array}{c}C_{V_{-} \text {vib }} \\
(\mathrm{J} / \mathrm{mol}-\mathrm{K})\end{array}$ & $\begin{array}{c}C_{V} \\
(\mathrm{~J} / \mathrm{mol}-\mathrm{K})\end{array}$ & $\begin{array}{c}h_{\text {sens }}^{\text {ig }}-R T \\
(\mathrm{~kJ} / \mathrm{mol})\end{array}$ \\
\hline 273.15 & 12.4715 & 12.4715 & 44.5560 & 69.4987 & -1.7942 \\
283.15 & 12.4715 & 12.4715 & 46.3670 & 71.3100 & -1.0902 \\
293.15 & 12.4715 & 12.4715 & 48.1890 & 73.1321 & -0.3679 \\
298.15 & 12.4715 & 12.4715 & 49.1030 & 74.0464 & 0.0000 \\
303.15 & 12.4715 & 12.4715 & 50.0190 & 74.9624 & 0.3725 \\
313.15 & 12.4715 & 12.4715 & 51.8550 & 76.7980 & 1.1313 \\
323.15 & 12.4715 & 12.4715 & 53.6930 & 78.6362 & 1.9085 \\
\hline
\end{tabular}

\subsubsection{MOLECULAR DYNAMicS}

With the ideal gas sensible enthalpy calculated, molecular dynamics simulations of the model compounds can be performed to determine the optimal values for the intermolecular parameters of the COMPASS model $(\sigma$ and $\varepsilon$ in the last term of equation (2)) for each atom type to most closely match experimental density and enthalpy data.

\section{Design of Experiments}

The creation of a DOE of molecular dynamics runs is performed with the goal of creating a response surface equation to approximate the design space. The design space being explored is of the density and specific heat as a function of the intermolecular parameter multipliers. Table 4.11 and Table 4.12 are the DOE's of molecular dynamics 
runs for norbornane and ethyl azide respectively in order to determine the sensitivity of density to changes in the intermolecular parameters. The multiplier values in these tables are multipliers of the baseline COMPASS intermolecular parameter values for each atom type of interest. These DOE run tables are created using the JMP Statistical Discovery software tool [57]. The baseline values are provided in Table 4.7 and Table 4.8 for norbornane and ethyl azide respectively.

Table 4.7: Norbornane Baseline COMPASS Intermolecular Parameter Values

\begin{tabular}{cccccc}
\hline$\sigma_{c 4}$ & $\varepsilon_{c 4}$ & $\sigma_{c 43}$ & $\varepsilon_{c 43}$ & $\sigma_{h 1}$ & $\varepsilon_{h 1}$ \\
\hline 3.854 & 0.062 & 3.854 & 0.040 & 2.878 & 0.023 \\
\hline
\end{tabular}

Table 4.8: Ethyl Azide Baseline COMPASS Intermolecular Parameter Values

\begin{tabular}{cccccccccc}
\hline$\sigma_{n 1 z}$ & $\varepsilon_{n 1 z}$ & $\sigma_{n 2 t}$ & $\varepsilon_{n 2 t}$ & $\sigma_{n 2 z}$ & $\varepsilon_{n 2 z}$ & $\sigma_{c 4 z}$ & $\varepsilon_{c 4 z}$ & $\sigma_{h 1}$ & $\varepsilon_{h 1}$ \\
\hline 3.520 & 0.085 & 3.300 & 0.050 & 3.400 & 0.120 & 3.650 & 0.080 & 2.878 & 0.023 \\
\hline
\end{tabular}

Table 4.9 and Table 4.10 provide the baseline COMPASS model charge bond increments for each bond type for norbornane and ethyl azide respectively. The charge bond increments are used to compute the charge associated with each atom in a molecule.

Table 4.9: Norbornane Baseline COMPASS Charge Bond Increments

\begin{tabular}{cccc}
\hline$\delta_{c 4-c 43}$ & $\delta_{c 4-c 4}$ & $\delta_{c 4-h 1}$ & $\delta_{c 43-h 1}$ \\
\hline 0.0000 & 0.0000 & -0.0530 & -0.0530 \\
\hline
\end{tabular}

Table 4.10: Ethyl Azide Baseline COMPASS Charge Bond Increments

\begin{tabular}{cccccc}
\hline$\delta_{\text {n1z-n2t }}$ & $\delta_{\text {n2t-n2z }}$ & $\delta_{\text {n2z-c4z }}$ & $\delta_{\text {c4z-c4 }}$ & $\delta_{\text {c4t-h1 }}$ & $\delta_{\text {c4-h1 }}$ \\
\hline-0.3860 & 0.2470 & -0.3110 & 0.0000 & -0.0530 & -0.0530 \\
\hline
\end{tabular}


Equation (73) describes how the charge associated with each atom is calculated from the charge bond increments. In equation (73), the charge for the $i^{\text {th }}$ atom in the molecule is calculated by summing the charge bond increments for the $n$ atoms bonded to atom $i$. For this research work, the charge bond increments were not adjusted in the intermolecular parameter optimization process. Discussion on the potential benefits of adjusting these parameters is provided in Chapter 6 .

$$
q_{i}=\sum_{j=1}^{n} \delta_{i j}
$$

All runs in a particular DOE for density are performed at the same temperature and pressure: $360 \mathrm{~K}$ and $1 \mathrm{~atm}$ for norbornane and $298.15 \mathrm{~K}$ and $1 \mathrm{~atm}$ for ethyl azide. This is done because experimental density data exists for liquid norbornane and liquid ethyl azide respectively at these temperatures and pressures. 
Table 4.11: Norbornane DOE - Intermolecular Parameter Multipliers and Results

\begin{tabular}{|c|c|c|c|c|c|c|c|}
\hline Run & $\sigma_{c 4}$ & $\varepsilon_{c 4}$ & $\sigma_{c 43}$ & $\varepsilon_{c 43}$ & $\sigma_{h 1}$ & $\varepsilon_{h 1}$ & $\begin{array}{l}\text { Density } \\
\left(\mathrm{kg} / \mathrm{m}^{3}\right)\end{array}$ \\
\hline 1 & 0.75 & 0.75 & 0.875 & 0.8 & 0.75 & 1.3 & 6.6 \\
\hline 2 & 0.75 & 0.75 & 0.875 & 0.8 & 1.25 & 0.8 & 8.7 \\
\hline 3 & 0.75 & 0.75 & 0.875 & 1.3 & 0.75 & 0.8 & 5.8 \\
\hline 4 & 0.75 & 0.75 & 0.875 & 1.3 & 1.25 & 1.3 & 510.2 \\
\hline 5 & 0.75 & 0.75 & 1.25 & 0.8 & 0.75 & 0.8 & 386.9 \\
\hline 6 & 0.75 & 0.75 & 1.25 & 0.8 & 1.25 & 1.3 & 615.1 \\
\hline 7 & 0.75 & 0.75 & 1.25 & 1.3 & 0.75 & 1.3 & 675.9 \\
\hline 8 & 0.75 & 0.75 & 1.25 & 1.3 & 1.25 & 0.8 & 646.2 \\
\hline 9 & 0.75 & 1.25 & 0.75 & 0.8 & 0.75 & 0.8 & 6.8 \\
\hline 10 & 0.75 & 1.25 & 0.75 & 0.8 & 1.25 & 1.3 & 9.0 \\
\hline 11 & 0.75 & 1.25 & 0.75 & 1.3 & 0.75 & 1.3 & 6.7 \\
\hline 12 & 0.75 & 1.25 & 0.75 & 1.3 & 1.25 & 0.8 & 9.0 \\
\hline 13 & 0.75 & 1.25 & 1.25 & 0.8 & 0.75 & 1.3 & 499.7 \\
\hline 14 & 0.75 & 1.25 & 1.25 & 0.8 & 1.25 & 0.8 & 553.9 \\
\hline 15 & 0.75 & 1.25 & 1.25 & 1.3 & 0.75 & 0.8 & 659.7 \\
\hline 16 & 0.75 & 1.25 & 1.25 & 1.3 & 1.25 & 1.3 & 646.7 \\
\hline 17 & 1.25 & 0.75 & 0.75 & 0.8 & 0.75 & 0.8 & 6.3 \\
\hline 18 & 1.25 & 0.75 & 0.75 & 0.8 & 1.25 & 1.3 & 381.3 \\
\hline 19 & 1.25 & 0.75 & 0.75 & 1.3 & 0.75 & 1.3 & 7.5 \\
\hline 20 & 1.25 & 0.75 & 0.75 & 1.3 & 1.25 & 0.8 & 7.9 \\
\hline 21 & 1.25 & 0.75 & 1.25 & 0.8 & 0.75 & 1.3 & 551.6 \\
\hline 22 & 1.25 & 0.75 & 1.25 & 0.8 & 1.25 & 0.8 & 586.8 \\
\hline 23 & 1.25 & 0.75 & 1.25 & 1.3 & 0.75 & 0.8 & 678.7 \\
\hline 24 & 1.25 & 0.75 & 1.25 & 1.3 & 1.25 & 1.3 & 656.3 \\
\hline 25 & 1.25 & 1.25 & 0.75 & 0.8 & 0.75 & 1.3 & 6.1 \\
\hline 26 & 1.25 & 1.25 & 0.75 & 0.8 & 1.25 & 0.8 & 7.2 \\
\hline 27 & 1.25 & 1.25 & 0.75 & 1.3 & 0.75 & 0.8 & 7.4 \\
\hline 28 & 1.25 & 1.25 & 0.75 & 1.3 & 1.25 & 1.3 & 476.8 \\
\hline 29 & 1.25 & 1.25 & 1.25 & 0.8 & 0.75 & 0.8 & 573.6 \\
\hline 30 & 1.25 & 1.25 & 1.25 & 0.8 & 1.25 & 1.3 & 642.0 \\
\hline 31 & 1.25 & 1.25 & 1.25 & 1.3 & 0.75 & 1.3 & 703.9 \\
\hline 32 & 1.25 & 1.25 & 1.25 & 1.3 & 1.25 & 0.8 & 668.2 \\
\hline 33 & 1.25 & 1 & 1 & 1 & 1 & 1 & 664.0 \\
\hline 34 & 0.75 & 1 & 1 & 1 & 1 & 1 & 630.9 \\
\hline 35 & 1. & 1.25 & 1. & 1 & 1 & 1 & 699.2 \\
\hline 36 & 1 & 0.75 & 1 & 1 & 1 & 1 & 658.3 \\
\hline 37 & 1 & 1 & 1.25 & 1 & 1 & 1 & 656.2 \\
\hline 38 & 1 & 1 & 0.875 & 1 & 1 & 1 & 6.5 \\
\hline 39 & 1 & 1 & 1 & 1.25 & 1 & 1 & 746.7 \\
\hline 40 & 1 & 1 & 1 & 0.75 & 1 & 1 & 584.4 \\
\hline 41 & 1 & 1 & 1 & 1 & 1.25 & 1 & 568.4 \\
\hline 42 & 1 & 1 & 1 & 1 & 0.75 & 1 & 626.2 \\
\hline 43 & 1 & 1 & 1 & 1 & 1 & 1.25 & 719.8 \\
\hline 44 & 1 & 1 & 1 & 1 & 1 & 0.75 & 633.0 \\
\hline 45 & 1 & 1 & 1 & 1 & 1 & 1 & 692.9 \\
\hline 46 & 1 & 1 & 1 & 1 & 1 & 1 & 689.5 \\
\hline 47 & 1 & 1 & 1 & 1 & 1 & 1 & 683.1 \\
\hline 48 & 1 & 1 & 1 & 1.25 & 0.75 & 1.25 & 802.3 \\
\hline 49 & 1 & 1 & 1 & 1.35 & 0.65 & 1.35 & 808.6 \\
\hline 50 & 1 & 1 & 1 & 1.45 & 0.55 & 1.45 & 811.4 \\
\hline
\end{tabular}


Table 4.12: Ethyl Azide DOE - Intermolecular Parameter Multipliers and Density

\begin{tabular}{|c|c|c|c|c|c|c|c|c|c|c|c|}
\hline Run & $\sigma_{n 1 z}$ & $\varepsilon_{n 1 z}$ & $\sigma_{n 2 t}$ & $\varepsilon_{n 2 t}$ & $\sigma_{n 2 z}$ & $\varepsilon_{n 2 z}$ & $\sigma_{c 4 z}$ & $\varepsilon_{C 4 z}$ & $\sigma_{h 1}$ & $\varepsilon_{h 1}$ & $\begin{array}{l}\text { Density } \\
\left(\mathrm{kg} / \mathrm{m}^{3}\right)\end{array}$ \\
\hline 1 & 0.8 & 0.8 & 0.8 & 0.8 & 0.8 & 0.8 & 0.8 & 0.8 & 1.2 & 1.2 & 762.3 \\
\hline 2 & 0.8 & 0.8 & 0.8 & 0.8 & 0.8 & 0.8 & 1.2 & 1.2 & 1.2 & 0.8 & 799.1 \\
\hline 3 & 0.8 & 0.8 & 0.8 & 0.8 & 0.8 & 1.2 & 0.8 & 1.2 & 0.8 & 1.2 & 1182.1 \\
\hline 4 & 0.8 & 0.8 & 0.8 & 0.8 & 0.8 & 1.2 & 1.2 & 0.8 & 0.8 & 0.8 & 915.2 \\
\hline 5 & 0.8 & 0.8 & 0.8 & 0.8 & 1.2 & 0.8 & 0.8 & 1.2 & 0.8 & 0.8 & 836.4 \\
\hline 6 & 0.8 & 0.8 & 0.8 & 0.8 & 1.2 & 0.8 & 1.2 & 0.8 & 0.8 & 1.2 & 852.3 \\
\hline 7 & 0.8 & 0.8 & 0.8 & 0.8 & 1.2 & 1.2 & 0.8 & 0.8 & 1.2 & 0.8 & 688.5 \\
\hline 8 & 0.8 & 0.8 & 0.8 & 0.8 & 1.2 & 1.2 & 1.2 & 1.2 & 1.2 & 1.2 & 821.8 \\
\hline 9 & 0.8 & 0.8 & 0.8 & 1.2 & 0.8 & 0.8 & 0.8 & 1.2 & 0.8 & 0.8 & 1127.8 \\
\hline 10 & 0.8 & 0.8 & 0.8 & 1.2 & 0.8 & 0.8 & 1.2 & 0.8 & 0.8 & 1.2 & 966.3 \\
\hline 11 & 0.8 & 0.8 & 0.8 & 1.2 & 0.8 & 1.2 & 0.8 & 0.8 & 1.2 & 0.8 & 713.8 \\
\hline 12 & 0.8 & 0.8 & 0.8 & 1.2 & 0.8 & 1.2 & 1.2 & 1.2 & 1.2 & 1.2 & 863.1 \\
\hline 13 & 0.8 & 0.8 & 0.8 & 1.2 & 1.2 & 0.8 & 0.8 & 0.8 & 1.2 & 1.2 & 720.0 \\
\hline 14 & 0.8 & 0.8 & 0.8 & 1.2 & 1.2 & 0.8 & 1.2 & 1.2 & 1.2 & 0.8 & 778.5 \\
\hline 15 & 0.8 & 0.8 & 0.8 & 1.2 & 1.2 & 1.2 & 0.8 & 1.2 & 0.8 & 1.2 & 909.9 \\
\hline 16 & 0.8 & 0.8 & 0.8 & 1.2 & 1.2 & 1.2 & 1.2 & 0.8 & 0.8 & 0.8 & 871.5 \\
\hline 17 & 0.8 & 0.8 & 1.2 & 0.8 & 0.8 & 0.8 & 0.8 & 1.2 & 0.8 & 0.8 & 870.5 \\
\hline 18 & 0.8 & 0.8 & 1.2 & 0.8 & 0.8 & 0.8 & 1.2 & 0.8 & 0.8 & 1.2 & 845.2 \\
\hline 19 & 0.8 & 0.8 & 1.2 & 0.8 & 0.8 & 1.2 & 0.8 & 0.8 & 1.2 & 0.8 & 661.5 \\
\hline 20 & 0.8 & 0.8 & 1.2 & 0.8 & 0.8 & 1.2 & 1.2 & 1.2 & 1.2 & 1.2 & 805.6 \\
\hline 21 & 0.8 & 0.8 & 1.2 & 0.8 & 1.2 & 0.8 & 0.8 & 0.8 & 1.2 & 1.2 & 714.6 \\
\hline 22 & 0.8 & 0.8 & 1.2 & 0.8 & 1.2 & 0.8 & 1.2 & 1.2 & 1.2 & 0.8 & 781.4 \\
\hline 23 & 0.8 & 0.8 & 1.2 & 0.8 & 1.2 & 1.2 & 0.8 & 1.2 & 0.8 & 1.2 & 885.0 \\
\hline 24 & 0.8 & 0.8 & 1.2 & 0.8 & 1.2 & 1.2 & 1.2 & 0.8 & 0.8 & 0.8 & 872.0 \\
\hline 25 & 0.8 & 0.8 & 1.2 & 1.2 & 0.8 & 0.8 & 0.8 & 0.8 & 1.2 & 1.2 & 704.7 \\
\hline 26 & 0.8 & 0.8 & 1.2 & 1.2 & 0.8 & 0.8 & 1.2 & 1.2 & 1.2 & 0.8 & 781.7 \\
\hline 27 & 0.8 & 0.8 & 1.2 & 1.2 & 0.8 & 1.2 & 0.8 & 1.2 & 0.8 & 1.2 & 958.4 \\
\hline 28 & 0.8 & 0.8 & 1.2 & 1.2 & 0.8 & 1.2 & 1.2 & 0.8 & 0.8 & 0.8 & 860.6 \\
\hline 29 & 0.8 & 0.8 & 1.2 & 1.2 & 1.2 & 0.8 & 0.8 & 1.2 & 0.8 & 0.8 & 856.8 \\
\hline 30 & 0.8 & 0.8 & 1.2 & 1.2 & 1.2 & 0.8 & 1.2 & 0.8 & 0.8 & 1.2 & 877.5 \\
\hline 31 & 0.8 & 0.8 & 1.2 & 1.2 & 1.2 & 1.2 & 0.8 & 0.8 & 1.2 & 0.8 & 728.3 \\
\hline 32 & 0.8 & 0.8 & 1.2 & 1.2 & 1.2 & 1.2 & 1.2 & 1.2 & 1.2 & 1.2 & 826.2 \\
\hline 33 & 0.8 & 1.2 & 0.8 & 0.8 & 0.8 & 0.8 & 0.8 & 1.2 & 0.8 & 0.8 & 1125.0 \\
\hline 34 & 0.8 & 1.2 & 0.8 & 0.8 & 0.8 & 0.8 & 1.2 & 0.8 & 0.8 & 1.2 & 988.4 \\
\hline 35 & 0.8 & 1.2 & 0.8 & 0.8 & 0.8 & 1.2 & 0.8 & 0.8 & 1.2 & 0.8 & 717.8 \\
\hline 36 & 0.8 & 1.2 & 0.8 & 0.8 & 0.8 & 1.2 & 1.2 & 1.2 & 1.2 & 1.2 & 869.9 \\
\hline 37 & 0.8 & 1.2 & 0.8 & 0.8 & 1.2 & 0.8 & 0.8 & 0.8 & 1.2 & 1.2 & 717.6 \\
\hline 38 & 0.8 & 1.2 & 0.8 & 0.8 & 1.2 & 0.8 & 1.2 & 1.2 & 1.2 & 0.8 & 790.6 \\
\hline 39 & 0.8 & 1.2 & 0.8 & 0.8 & 1.2 & 1.2 & 0.8 & 1.2 & 0.8 & 1.2 & 910.7 \\
\hline 40 & 0.8 & 1.2 & 0.8 & 0.8 & 1.2 & 1.2 & 1.2 & 0.8 & 0.8 & 0.8 & 863.0 \\
\hline 41 & 0.8 & 1.2 & 0.8 & 1.2 & 0.8 & 0.8 & 0.8 & 0.8 & 1.2 & 1.2 & 778.4 \\
\hline 42 & 0.8 & 1.2 & 0.8 & 1.2 & 0.8 & 0.8 & 1.2 & 1.2 & 1.2 & 0.8 & 836.2 \\
\hline 43 & 0.8 & 1.2 & 0.8 & 1.2 & 0.8 & 1.2 & 0.8 & 1.2 & 0.8 & 1.2 & 1186.4 \\
\hline 44 & 0.8 & 1.2 & 0.8 & 1.2 & 0.8 & 1.2 & 1.2 & 0.8 & 0.8 & 0.8 & 968.0 \\
\hline 45 & 0.8 & 1.2 & 0.8 & 1.2 & 1.2 & 0.8 & 0.8 & 1.2 & 0.8 & 0.8 & 850.6 \\
\hline 46 & 0.8 & 1.2 & 0.8 & 1.2 & 1.2 & 0.8 & 1.2 & 0.8 & 0.8 & 1.2 & 853.6 \\
\hline 47 & 0.8 & 1.2 & 0.8 & 1.2 & 1.2 & 1.2 & 0.8 & 0.8 & 1.2 & 0.8 & 704.7 \\
\hline 48 & 0.8 & 1.2 & 0.8 & 1.2 & 1.2 & 1.2 & 1.2 & 1.2 & 1.2 & 1.2 & 834.4 \\
\hline 49 & 0.8 & 1.2 & 1.2 & 0.8 & 0.8 & 0.8 & 0.8 & 0.8 & 1.2 & 1.2 & 703.5 \\
\hline 50 & 0.8 & 1.2 & 1.2 & 0.8 & 0.8 & 0.8 & 1.2 & 1.2 & 1.2 & 0.8 & 773.9 \\
\hline
\end{tabular}


Table 4.12 (continued)

\begin{tabular}{|c|c|c|c|c|c|c|c|c|c|c|c|}
\hline 51 & 0.8 & 1.2 & 1.2 & 0.8 & 0.8 & 1.2 & 0.8 & 1.2 & 0.8 & 1.2 & 969.6 \\
\hline 52 & 0.8 & 1.2 & 1.2 & 0.8 & 0.8 & 1.2 & 1.2 & 0.8 & 0.8 & 0.8 & 851.7 \\
\hline 53 & 0.8 & 1.2 & 1.2 & 0.8 & 1.2 & 0.8 & 0.8 & 1.2 & 0.8 & 0.8 & 843.8 \\
\hline 54 & 0.8 & 1.2 & 1.2 & 0.8 & 1.2 & 0.8 & 1.2 & 0.8 & 0.8 & 1.2 & 839.8 \\
\hline 55 & 0.8 & 1.2 & 1.2 & 0.8 & 1.2 & 1.2 & 0.8 & 0.8 & 1.2 & 0.8 & 74.5 \\
\hline 56 & 0.8 & 1.2 & 1.2 & 0.8 & 1.2 & 1.2 & 1.2 & 1.2 & 1.2 & 1.2 & 821.9 \\
\hline 57 & 0.8 & 1.2 & 1.2 & 1.2 & 0.8 & 0.8 & 0.8 & 1.2 & 0.8 & 0.8 & 925.7 \\
\hline 58 & 0.8 & 1.2 & 1.2 & 1.2 & 0.8 & 0.8 & 1.2 & 0.8 & 0.8 & 1.2 & 881.0 \\
\hline 59 & 0.8 & 1.2 & 1.2 & 1.2 & 0.8 & 1.2 & 0.8 & 0.8 & 1.2 & 0.8 & 685.2 \\
\hline 60 & 0.8 & 1.2 & 1.2 & 1.2 & 0.8 & 1.2 & 1.2 & 1.2 & 1.2 & 1.2 & 824.2 \\
\hline 61 & 0.8 & 1.2 & 1.2 & 1.2 & 1.2 & 0.8 & 0.8 & 0.8 & 1.2 & 1.2 & 729.9 \\
\hline 62 & 0.8 & 1.2 & 1.2 & 1.2 & 1.2 & 0.8 & 1.2 & 1.2 & 1.2 & 0.8 & 798.8 \\
\hline 63 & 0.8 & 1.2 & 1.2 & 1.2 & 1.2 & 1.2 & 0.8 & 1.2 & 0.8 & 1.2 & 917.8 \\
\hline 64 & 0.8 & 1.2 & 1.2 & 1.2 & 1.2 & 1.2 & 1.2 & 0.8 & 0.8 & 0.8 & 894.3 \\
\hline 65 & 1.2 & 0.8 & 0.8 & 0.8 & 0.8 & 0.8 & 0.8 & 1.2 & 0.8 & 0.8 & 344.5 \\
\hline 66 & 1.2 & 0.8 & 0.8 & 0.8 & 0.8 & 0.8 & 1.2 & 0.8 & 0.8 & 1.2 & 589.2 \\
\hline 67 & 1.2 & 0.8 & 0.8 & 0.8 & 0.8 & 1.2 & 0.8 & 0.8 & 1.2 & 0.8 & 428.8 \\
\hline 68 & 1.2 & 0.8 & 0.8 & 0.8 & 0.8 & 1.2 & 1.2 & 1.2 & 1.2 & 1.2 & 660.2 \\
\hline 69 & 1.2 & 0.8 & 0.8 & 0.8 & 1.2 & 0.8 & 0.8 & 0.8 & 1.2 & 1.2 & 568.8 \\
\hline 70 & 1.2 & 0.8 & 0.8 & 0.8 & 1.2 & 0.8 & 1.2 & 1.2 & 1.2 & 0.8 & 643.7 \\
\hline 71 & 1.2 & 0.8 & 0.8 & 0.8 & 1.2 & 1.2 & 0.8 & 1.2 & 0.8 & 1.2 & 628.1 \\
\hline 72 & 1.2 & 0.8 & 0.8 & 0.8 & 1.2 & 1.2 & 1.2 & 0.8 & 0.8 & 0.8 & 667.4 \\
\hline 73 & 1.2 & 0.8 & 0.8 & 1.2 & 0.8 & 0.8 & 0.8 & 0.8 & 1.2 & 1.2 & 520.2 \\
\hline 74 & 1.2 & 0.8 & 0.8 & 1.2 & 0.8 & 0.8 & 1.2 & 1.2 & 1.2 & 0.8 & 622.3 \\
\hline 75 & 1.2 & 0.8 & 0.8 & 1.2 & 0.8 & 1.2 & 0.8 & 1.2 & 0.8 & 1.2 & 638.9 \\
\hline 76 & 1.2 & 0.8 & 0.8 & 1.2 & 0.8 & 1.2 & 1.2 & 0.8 & 0.8 & 0.8 & 552.7 \\
\hline 77 & 1.2 & 0.8 & 0.8 & 1.2 & 1.2 & 0.8 & 0.8 & 1.2 & 0.8 & 0.8 & 523.6 \\
\hline 78 & 1.2 & 0.8 & 0.8 & 1.2 & 1.2 & 0.8 & 1.2 & 0.8 & 0.8 & 1.2 & 635.6 \\
\hline 79 & 1.2 & 0.8 & 0.8 & 1.2 & 1.2 & 1.2 & 0.8 & 0.8 & 1.2 & 0.8 & 550.4 \\
\hline 80 & 1.2 & 0.8 & 0.8 & 1.2 & 1.2 & 1.2 & 1.2 & 1.2 & 1.2 & 1.2 & 688.1 \\
\hline 81 & 1.2 & 0.8 & 1.2 & 0.8 & 0.8 & 0.8 & 0.8 & 0.8 & 1.2 & 1.2 & 586.7 \\
\hline 82 & 1.2 & 0.8 & 1.2 & 0.8 & 0.8 & 0.8 & 1.2 & 1.2 & 1.2 & 0.8 & 656.9 \\
\hline 83 & 1.2 & 0.8 & 1.2 & 0.8 & 0.8 & 1.2 & 0.8 & 1.2 & 0.8 & 1.2 & 660.7 \\
\hline 84 & 1.2 & 0.8 & 1.2 & 0.8 & 0.8 & 1.2 & 1.2 & 0.8 & 0.8 & 0.8 & 660.0 \\
\hline 85 & 1.2 & 0.8 & 1.2 & 0.8 & 1.2 & 0.8 & 0.8 & 1.2 & 0.8 & 0.8 & 648.5 \\
\hline 86 & 1.2 & 0.8 & 1.2 & 0.8 & 1.2 & 0.8 & 1.2 & 0.8 & 0.8 & 1.2 & 711.8 \\
\hline 87 & 1.2 & 0.8 & 1.2 & 0.8 & 1.2 & 1.2 & 0.8 & 0.8 & 1.2 & 0.8 & 615.0 \\
\hline 88 & 1.2 & 0.8 & 1.2 & 0.8 & 1.2 & 1.2 & 1.2 & 1.2 & 1.2 & 1.2 & 717.2 \\
\hline 89 & 1.2 & 0.8 & 1.2 & 1.2 & 0.8 & 0.8 & 0.8 & 1.2 & 0.8 & 0.8 & 662.8 \\
\hline 90 & 1.2 & 0.8 & 1.2 & 1.2 & 0.8 & 0.8 & 1.2 & 0.8 & 0.8 & 1.2 & 685.4 \\
\hline 91 & 1.2 & 0.8 & 1.2 & 1.2 & 0.8 & 1.2 & 0.8 & 0.8 & 1.2 & 0.8 & 581.3 \\
\hline 92 & 1.2 & 0.8 & 1.2 & 1.2 & 0.8 & 1.2 & 1.2 & 1.2 & 1.2 & 1.2 & 693.7 \\
\hline 93 & 1.2 & 0.8 & 1.2 & 1.2 & 1.2 & 0.8 & 0.8 & 0.8 & 1.2 & 1.2 & 638.7 \\
\hline 94 & 1.2 & 0.8 & 1.2 & 1.2 & 1.2 & 0.8 & 1.2 & 1.2 & 1.2 & 0.8 & 697.7 \\
\hline 95 & 1.2 & 0.8 & 1.2 & 1.2 & 1.2 & 1.2 & 0.8 & 1.2 & 0.8 & 1.2 & 733.9 \\
\hline 96 & 1.2 & 0.8 & 1.2 & 1.2 & 1.2 & 1.2 & 1.2 & 0.8 & 0.8 & 0.8 & 745.9 \\
\hline 97 & 1.2 & 1.2 & 0.8 & 0.8 & 0.8 & 0.8 & 0.8 & 0.8 & 1.2 & 1.2 & 545.4 \\
\hline 98 & 1.2 & 1.2 & 0.8 & 0.8 & 0.8 & 0.8 & 1.2 & 1.2 & 1.2 & 0.8 & 632.1 \\
\hline 99 & 1.2 & 1.2 & 0.8 & 0.8 & 0.8 & 1.2 & 0.8 & 1.2 & 0.8 & 1.2 & 646.7 \\
\hline 100 & 1.2 & 1.2 & 0.8 & 0.8 & 0.8 & 1.2 & 1.2 & 0.8 & 0.8 & 0.8 & 611.0 \\
\hline 101 & 1.2 & 1.2 & 0.8 & 0.8 & 1.2 & 0.8 & 0.8 & 1.2 & 0.8 & 0.8 & 522.1 \\
\hline 102 & 1.2 & 1.2 & 0.8 & 0.8 & 1.2 & 0.8 & 1.2 & 0.8 & 0.8 & 1.2 & 647.0 \\
\hline 103 & 1.2 & 1.2 & 0.8 & 0.8 & 1.2 & 1.2 & 0.8 & 0.8 & 1.2 & 0.8 & 587.9 \\
\hline 104 & 1.2 & 1.2 & 0.8 & 0.8 & 1.2 & 1.2 & 1.2 & 1.2 & 1.2 & 1.2 & 688.6 \\
\hline
\end{tabular}


Table 4.12 (continued)

\begin{tabular}{|c|c|c|c|c|c|c|c|c|c|c|c|}
\hline 105 & 1.2 & 1.2 & 0.8 & 1.2 & 0.8 & 0.8 & 0.8 & 1.2 & 0.8 & 0.8 & 572.2 \\
\hline 106 & 1.2 & 1.2 & 0.8 & 1.2 & 0.8 & 0.8 & 1.2 & 0.8 & 0.8 & 1.2 & 665.9 \\
\hline 107 & 1.2 & 1.2 & 0.8 & 1.2 & 0.8 & 1.2 & 0.8 & 0.8 & 1.2 & 0.8 & 517.0 \\
\hline 108 & 1.2 & 1.2 & 0.8 & 1.2 & 0.8 & 1.2 & 1.2 & 1.2 & 1.2 & 1.2 & 688.4 \\
\hline 109 & 1.2 & 1.2 & 0.8 & 1.2 & 1.2 & 0.8 & 0.8 & 0.8 & 1.2 & 1.2 & 582.3 \\
\hline 110 & 1.2 & 1.2 & 0.8 & 1.2 & 1.2 & 0.8 & 1.2 & 1.2 & 1.2 & 0.8 & 663.1 \\
\hline 111 & 1.2 & 1.2 & 0.8 & 1.2 & 1.2 & 1.2 & 0.8 & 1.2 & 0.8 & 1.2 & 647.5 \\
\hline 112 & 1.2 & 1.2 & 0.8 & 1.2 & 1.2 & 1.2 & 1.2 & 0.8 & 0.8 & 0.8 & 695.6 \\
\hline 113 & 1.2 & 1.2 & 1.2 & 0.8 & 0.8 & 0.8 & 0.8 & 1.2 & 0.8 & 0.8 & 657.6 \\
\hline 114 & 1.2 & 1.2 & 1.2 & 0.8 & 0.8 & 0.8 & 1.2 & 0.8 & 0.8 & 1.2 & 694.9 \\
\hline 115 & 1.2 & 1.2 & 1.2 & 0.8 & 0.8 & 1.2 & 0.8 & 0.8 & 1.2 & 0.8 & 581.3 \\
\hline 116 & 1.2 & 1.2 & 1.2 & 0.8 & 0.8 & 1.2 & 1.2 & 1.2 & 1.2 & 1.2 & 699.9 \\
\hline 117 & 1.2 & 1.2 & 1.2 & 0.8 & 1.2 & 0.8 & 0.8 & 0.8 & 1.2 & 1.2 & 622.8 \\
\hline 118 & 1.2 & 1.2 & 1.2 & 0.8 & 1.2 & 0.8 & 1.2 & 1.2 & 1.2 & 0.8 & 692.6 \\
\hline 119 & 1.2 & 1.2 & 1.2 & 0.8 & 1.2 & 1.2 & 0.8 & 1.2 & 0.8 & 1.2 & 726.4 \\
\hline 120 & 1.2 & 1.2 & 1.2 & 0.8 & 1.2 & 1.2 & 1.2 & 0.8 & 0.8 & 0.8 & 742.1 \\
\hline 121 & 1.2 & 1.2 & 1.2 & 1.2 & 0.8 & 0.8 & 0.8 & 0.8 & 1.2 & 1.2 & 608.0 \\
\hline 122 & 1.2 & 1.2 & 1.2 & 1.2 & 0.8 & 0.8 & 1.2 & 1.2 & 1.2 & 0.8 & 686.5 \\
\hline 123 & 1.2 & 1.2 & 1.2 & 1.2 & 0.8 & 1.2 & 0.8 & 1.2 & 0.8 & 1.2 & 736.9 \\
\hline 124 & 1.2 & 1.2 & 1.2 & 1.2 & 0.8 & 1.2 & 1.2 & 0.8 & 0.8 & 0.8 & 742.0 \\
\hline 125 & 1.2 & 1.2 & 1.2 & 1.2 & 1.2 & 0.8 & 0.8 & 1.2 & 0.8 & 0.8 & 703.5 \\
\hline 126 & 1.2 & 1.2 & 1.2 & 1.2 & 1.2 & 0.8 & 1.2 & 0.8 & 0.8 & 1.2 & 739.9 \\
\hline 127 & 1.2 & 1.2 & 1.2 & 1.2 & 1.2 & 1.2 & 0.8 & 0.8 & 1.2 & 0.8 & 643.5 \\
\hline 128 & 1.2 & 1.2 & 1.2 & 1.2 & 1.2 & 1.2 & 1.2 & 1.2 & 1.2 & 1.2 & 733.8 \\
\hline 129 & 0.8 & 1 & 1 & 1 & 1 & 1 & 1 & 1 & 1 & 1 & 988.0 \\
\hline 130 & 1.2 & 1 & 1 & 1. & 1 & 1 & 1 & 1 & 1 & 1. & 728.8 \\
\hline 131 & 1 & 0.8 & 1 & 1 & 1 & 1 & 1 & 1 & 1 & 1 & 875.8 \\
\hline 132 & 1 & 1.2 & 1 & 1 & 1 & 1 & 1 & 1 & 1 & 1 & 900.3 \\
\hline 133 & 1 & 1 & 0.8 & 1 & 1 & 1 & 1 & 1 & 1 & 1 & 874.6 \\
\hline 134 & 1 & 1 & 1.2 & 1 & 1 & 1 & 1 & 1 & 1 & 1 & 870.0 \\
\hline 135 & 1 & 1 & 1 & 0.8 & 1 & 1 & 1 & 1 & 1 & 1 & 882.1 \\
\hline 136 & 1 & 1 & 1 & 1.2 & 1 & 1 & 1 & 1 & 1 & 1 & 910.2 \\
\hline 137 & 1 & 1 & 1 & 1 & 0.8 & 1 & 1 & 1 & 1 & 1 & 891.0 \\
\hline 138 & 1 & 1 & 1 & 1 & 1.2 & 1 & 1 & 1 & 1 & 1 & 840.6 \\
\hline 139 & 1 & 1 & 1 & 1 & 1 & 0.8 & 1 & 1 & 1 & 1 & 883.5 \\
\hline 140 & 1 & 1 & 1 & 1 & 1 & 1.2 & 1 & 1 & 1 & 1 & 909.9 \\
\hline 141 & 1 & 1 & 1 & 1 & 1 & 1 & 0.8 & 1 & 1 & 1 & 845.5 \\
\hline 142 & 1 & 1 & 1 & 1 & 1 & 1 & 1.2 & 1 & 1 & 1 & 868.5 \\
\hline 143 & 1 & 1 & 1 & 1 & 1 & 1 & 1 & 0.8 & 1 & 1 & 871.7 \\
\hline 144 & 1 & 1 & 1 & 1 & 1 & 1 & 1 & 1.2 & 1 & 1 & 907.2 \\
\hline 145 & 1 & 1 & 1 & 1 & 1 & 1 & 1 & 1 & 0.8 & 1 & 929.2 \\
\hline 146 & 1 & 1 & 1 & 1 & 1 & 1 & 1 & 1 & 1.2 & 1 & 782.6 \\
\hline 147 & 1 & 1 & 1 & 1 & 1 & 1 & 1 & 1 & 1 & 0.8 & 886.1 \\
\hline 148 & 1 & 1 & 1 & 1 & 1 & 1 & 1 & 1 & 1 & 1.2 & 898.6 \\
\hline 149 & 1 & 1 & 1 & 1 & 1 & 1 & 1 & 1 & 1 & 1 & 891.7 \\
\hline 150 & 1. & 1 & 1. & 1. & 1 & 1 & 1 & 1 & 1 & 1. & 885.7 \\
\hline 151 & 1 & 1 & 1 & 1 & 1 & 1 & 1 & 1 & 1 & 1 & 901.4 \\
\hline
\end{tabular}


In addition to the above DOE's used to determine the sensitivity of density to changes in intermolecular parameters, the DOE's in Table 4.13 and Table 4.14 are used to determine the sensitivity of specific heat to changes in intermolecular parameters. These DOE's are different from the density DOE's because for a particular set of intermolecular parameter settings, two runs are performed at different temperatures in order to calculate the change in enthalpy as a function of temperature (to compute specific heat). For norbornane, molecular dynamics runs are performed at $360 \mathrm{~K}$ and 380 $\mathrm{K}$, both at $1 \mathrm{~atm}$. For ethyl azide, molecular dynamics runs are performed at $273.15 \mathrm{~K}$ and $323.15 \mathrm{~K}$, both at $1 \mathrm{~atm}$. These temperatures and pressures are chosen because there is experimental liquid data available.

The combination of the density and specific heat DOE's are used to create response surface equations to determine the optimal settings for the intermolecular parameters. In Table 4.13 and Table 4.14, the specific heat is calculated using equations (11) - (14). These equations are repeated here for convenience:

$$
\begin{gathered}
C_{P}(T, P)=C_{P}^{i g}(T)-R+C_{P}^{r e s}(T, P) \\
C_{P}^{i g}(T)-R=C_{V}^{t r}+C_{V}^{r o t}+C_{V}^{v i b}=\frac{3}{2} R+\frac{3}{2} R+\sum_{i=1}^{3 N-6} R\left(\frac{\theta_{v i b_{i}}}{T}\right)^{2} \frac{e^{\theta_{v i b_{i}} / T}}{\left(e^{\theta_{v i b_{i}} / T}-1\right)^{2}} \\
h_{\text {sens }}^{i g}-R T=\int_{298.15 K}^{T} C_{P}^{i g}(\tau) d \tau-R T \\
C_{P}^{r e s}(T, P)=\left(\frac{\partial\left\langle H^{r e s}(T, P)\right\rangle}{\partial T}\right)_{P}=\frac{\partial}{\partial T}\left(P E^{i n t e r}(T, P)+\frac{P}{\rho(T, P)}\right)_{P}
\end{gathered}
$$


Table 4.13: Norbornane DOE - Intermolecular Parameter Multipliers and $C_{P}$

\begin{tabular}{|c|c|c|c|c|c|c|c|c|}
\hline Run & $T(K)$ & $\sigma_{c 4}$ & $\varepsilon_{C 4}$ & $\sigma_{c 43}$ & $\varepsilon_{c 43}$ & $\sigma_{h 1}$ & $\varepsilon_{h 1}$ & $\begin{array}{c}C_{P} \\
(\mathrm{cal} / \mathrm{mol}-\mathrm{K})\end{array}$ \\
\hline 1 & 360 & 1.1 & 1 & 1 & 1 & 1 & 1 & 467537 \\
\hline 2 & 380 & 1.1 & 1 & 1. & 1. & 1 & 1. & 46.7537 \\
\hline 3 & 360 & 0.9 & 1 & 1 & 1 & 1 & 1 & \\
\hline 4 & 380 & 0.9 & 1 & 1 & 1 & 1 & 1 & 48.6133 \\
\hline 5 & 360 & 1 & 1.1 & 1 & 1 & 1 & 1 & \\
\hline 6 & 380 & 1 & 1.1 & 1 & 1 & 1 & 1 & 41.7002 \\
\hline 7 & 360 & 1 & 0.9 & 1 & 1 & 1 & 1 & \\
\hline 8 & 380 & 1 & 0.9 & 1 & 1 & 1 & 1 & 48.9731 \\
\hline 9 & 360 & 1 & 1 & 1.1 & 1 & 1 & 1 & \\
\hline 10 & 380 & 1 & 1 & 1.1 & 1 & 1 & 1 & $39.15 / 1$ \\
\hline 11 & 360 & 1 & 1 & $0.9^{-}$ & 1 & 1 & 1 & 61 \\
\hline 12 & 380 & 1 & 1 & 0.9 & 1 & 1 & 1 & 50.6061 \\
\hline 13 & 360 & 1 & 1 & 1 & 1.1 & 1 & 1 & 12.6254 \\
\hline 14 & 380 & 1 & 1 & 1 & 1.1 & 1 & 1 & 42.0354 \\
\hline 15 & 360 & 1 & 1 & 1 & 0.9 & 1 & 1 & 517772 \\
\hline 16 & 380 & 1 & 1 & 1. & 0.9 & 1 & 1 & ग1./1/L \\
\hline 17 & 360 & 1 & 1 & 1 & 1 & 1.1 & 1 & 502703 \\
\hline 18 & 380 & 1 & 1 & 1. & 1 & 1.1 & 1 & $50.2 / 93$ \\
\hline 19 & 360 & 1 & 1 & 1 & 1 & 0.9 & 1 & 402156 \\
\hline 20. & 380 & 1 & 1. & 1. & 1. & 0.9 & 1 & 40.2156 \\
\hline 21 & 360 & 1 & 1 & 1 & 1 & 1 & 1.1 & 384435 \\
\hline 22 & 380 & 1 & 1 & 1. & 1. & 1 & 1.1 & 38.4435 \\
\hline 23 & 360 & 1 & 1 & 1 & 1 & 1 & 0.9 & 480030 \\
\hline 24. & 380 & 1 & 1 & 1. & 1. & 1. & 0.9 & 48.0030 \\
\hline 25 & 360 & 1 & 1 & 1 & 1 & 1 & 1 & 78 \\
\hline 26 & 380 & 1 & 1 & 1 & 1 & 1 & 1 & $4 . .0211$ \\
\hline
\end{tabular}


Table 4.14: Ethyl Azide DOE - Intermolecular Parameter Multipliers and $C_{P}$

\begin{tabular}{|c|c|c|c|c|c|c|c|c|c|c|c|c|}
\hline Run & $T(K)$ & $\sigma_{n 1 z}$ & $\varepsilon_{n 1 z}$ & $\sigma_{n 2 t}$ & $\varepsilon_{n 2 t}$ & $\sigma_{n 2 z}$ & $\varepsilon_{n 2 z}$ & $\sigma_{c 4 z}$ & $\varepsilon_{C 4 z}$ & $\sigma_{h 1}$ & $\varepsilon_{h 1}$ & $\begin{array}{c}C_{P} \\
(\mathrm{cal} / \mathrm{mol}-\mathrm{K})\end{array}$ \\
\hline 1 & 273.15 & 1.2 & 1 & 1 & 1 & 1 & 1 & 1 & 1 & 1 & 1 & \\
\hline 2 & 323.15 & 1.2 & 1. & 1. & 1 & 1 & 1. & 1 & 1. & 1 & 1 & 32.5021 \\
\hline 3 & 273.15 & 0.8 & 1 & 1 & 1 & 1 & 1 & 1 & 1 & 1 & 1 & \\
\hline 4 & 323.15 & 0.8 & 1. & 1 & 1 & 1 & 1 & 1 & 1 & 1 & 1 & 36.4428 \\
\hline 5 & 273.15 & 1 & 1.2 & 1 & 1 & 1 & 1 & 1 & 1 & 1 & 1 & \\
\hline 6 & 323.15 & 1. & 1.2 & 1. & 1 & 1 & 1 & 1 & 1 & 1 & 1 & 35.8510 \\
\hline 7 & 273.15 & 1 & 0.8 & 1 & 1 & 1 & 1 & 1 & 1 & 1 & 1 & \\
\hline 8 & 323.15 & 1 & 0.8 & 1 & 1 & 1 & 1 & 1 & 1 & 1 & 1 & 36.1449 \\
\hline 9 & 273.15 & 1 & 1 & 1.2 & 1 & 1 & 1 & 1 & 1 & 1 & 1 & \\
\hline 10 & 323.15 & 1 & 1 & 1.2 & 1 & 1 & 1 & 1 & 1 & 1 & 1 & 34.9052 \\
\hline 11 & 273.15 & 1 & 1 & 0.8 & 1 & 1 & 1 & 1 & 1 & 1 & 1 & \\
\hline 12 & 323.15 & 1 & 1 & 0.8 & 1 & 1 & 1 & 1 & 1 & 1 & 1 & 34.1106 \\
\hline 13 & 273.15 & 1 & 1 & 1 & $1.2^{\circ}$ & 1 & 1 & 1 & 1 & 1 & 1 & \\
\hline 14 & 323.15 & 1 & 1 & 1 & 1.2 & 1 & 1 & 1 & 1 & 1 & 1 & 36.4420 \\
\hline 15 & 273.15 & 1 & 1 & 1 & 0.8 & 1 & 1 & 1 & 1 & 1 & 1 & \\
\hline 16 & 323.15 & 1 & 1 & 1 & 0.8 & 1 & 1. & 1 & 1. & 1 & 1 & 32.3643 \\
\hline 17 & 273.15 & 1 & 1 & 1 & 1 & 1.2 & 1 & 1 & 1 & 1 & 1 & 304584 \\
\hline 18 & 323.15 & 1 & 1 & 1 & 1 & 1.2 & 1 & 1 & 1 & 1 & 1 & 30.4584 \\
\hline 19 & 273.15 & 1 & 1 & 1 & 1 & 0.8 & 1 & 1 & 1 & 1 & 1 & \\
\hline 20 & 323.15 & 1. & 1. & 1. & 1. & 0.8 & 1. & 1 & 1. & 1 & 1 & 38.0530 \\
\hline 21 & 273.15 & 1 & 1 & 1 & 1 & 1 & 1.2 & 1 & 1 & 1 & 1 & \\
\hline 22 & 323.15 & 1 & 1 & 1 & 1 & 1 & 1.2 & 1 & 1 & 1 & 1 & 3.5489 \\
\hline 23 & 273.15 & 1 & 1 & 1 & 1 & 1 & 0.8 & 1 & 1 & 1 & 1 & \\
\hline 24 & 323.15 & 1 & 1 & 1 & 1 & 1 & 0.8 & 1 & 1 & 1 & 1 & $35.6 / 40$ \\
\hline 25 & 273.15 & 1 & 1 & 1 & 1 & 1 & 1 & 1.2 & 1 & 1 & 1 & 220786 \\
\hline 26 & 323.15 & 1 & 1 & 1 & 1 & 1 & 1 & 1.2 & 1 & 1 & 1 & 35.9286 \\
\hline 27 & 273.15 & 1 & 1 & 1 & 1 & 1 & 1 & 0.8 & 1 & 1 & 1 & \\
\hline 28 & 323.15 & 1 & 1 & 1 & 1 & 1 & 1 & 0.8 & 1 & 1 & 1 & \\
\hline 29 & 273.15 & 1 & 1 & 1 & 1 & 1 & 1 & 1 & 1.2 & 1 & 1 & \\
\hline 30 & 323.15 & 1. & 1 & 1 & 1 & 1 & 1 & 1 & 1.2 & 1 & 1 & 36.2093 \\
\hline 31 & 273.15 & 1 & 1 & 1 & 1 & 1 & 1 & 1 & 0.8 & 1 & 1 & 35.4663 \\
\hline 32 & 323.15 & 1 & 1 & 1 & 1 & 1 & 1 & 1 & 0.8 & 1 & 1 & 35.4663 \\
\hline 33 & 273.15 & 1 & 1 & 1 & 1 & 1 & 1 & 1 & 1 & 1.2 & 1 & 325348 \\
\hline 34. & 323.15 & 1. & 1. & 1. & 1 & 1 & 1. & 1 & 1. & 1.2 & 1 & 32.5348 \\
\hline 35 & 273.15 & 1 & 1 & 1 & 1 & 1 & 1 & 1 & 1 & 0.8 & 1 & \\
\hline 36 & 323.15 & 1 & 1 & 1 & 1 & 1 & 1 & 1 & 1 & 0.8 & 1 & 37.5660 \\
\hline 37 & 273.15 & 1 & 1 & 1 & 1 & 1 & 1 & 1 & 1 & 1 & 1.2 & 250160 \\
\hline 38 & 323.15 & 1 & 1 & 1 & 1 & 1 & 1 & 1 & 1 & 1 & 1.2 & 35.0169 \\
\hline 39 & 273.15 & 1 & 1 & 1 & 1 & 1 & 1 & 1 & 1 & 1 & 0.8 & 240177 \\
\hline 40 & 323.15 & 1 & 1 & 1 & 1 & 1 & 1 & 1 & 1 & 1 & 0.8 & $34.81 / 1 /$ \\
\hline 41 & 273.15 & 1 & 1 & 1 & 1 & 1 & 1 & 1 & 1 & 1 & 1 & 368872 \\
\hline 42 & 323.15 & 1 & 1 & 1 & 1 & 1 & 1 & 1 & 1 & 1 & 1 & $30.88 / 2$ \\
\hline
\end{tabular}




\section{Response Surface Equations}

With the DOE's completed, a response surface equation (RSE) can be created for both density and specific heat for each model compound. The RSE's are simply multidimensional polynomial curve fits of the relationship between density and specific heat and the intermolecular parameter multiplier settings. The RSE's are approximations of the design space that are used by an optimizer to determine the most appropriate settings for the intermolecular parameters to best match experimental density and specific heat data for each model compound.

In order to produce an accurate RSE for the density of norbornane, the low density gas phase DOE runs are first removed before fitting a RSE to the data. These removed runs, whose common trait is a $\sigma_{c 43}$ multiplier that is less than one, would adversely affect the accuracy of a RSE if left in the DOE. This is because the RSE is a simple quadratic approximation of the design space and the phase change from liquid to gas would be approximated very poorly. Leaving out these runs is acceptable as the goal of the RSE is to approximate the thermophysical properties in the condensed phase (the HEDM propellants will only exist in the condensed phase while flowing through the different engine components and break down into simpler hydrocarbons and other compounds before any potential phase change would occur).

The JMP Statistical Discovery software tool [57] is used to fit a RSE to each of the DOE tables. A separate RSE is fit for density and specific heat for each model compound (a total of four RSE's). Table 4.15 - Table 4.18 provide the RSE coefficients for each of the four RSE's. The form of each equation is slightly different as there are a different number of terms for each RSE and some of the coefficients use normalized values while others do not (specifically the $\sigma_{\mathrm{h} 1}$ and $\varepsilon_{\mathrm{h} 1}$ terms for ethyl azide do not use normalized values for $\sigma_{\mathrm{h} 1}$ and $\varepsilon_{\mathrm{h} 1}$ due to RSE limitations in JMP). The exact form of each RSE is provided after each table. 
It is important to note that the fitting of RSE's is a bit of an art. The choices made for the fitting of RSE's in this research project could be changed and a slightly different RSE created. The point of the creation of RSE's in this project is to create the best possible approximation of the results from molecular dynamics simulations. A slightly different RSE should not affect the overall process, other than possibly requiring a few more (or less) molecular dynamics DOE runs.

Table 4.15: Norbornane Density RSE Coefficients

\begin{tabular}{ccccc}
\hline$i$ & $j$ & $x_{i}$ & $x_{j}$ & $\beta_{\mathrm{ij}}$ \\
\hline 0 & & & & 689.85516 \\
1 & & $\sigma_{\mathrm{c} 4}$ & & 34.061695 \\
2 & & $\varepsilon_{\mathrm{c} 4}$ & & 26.173528 \\
3 & & $\sigma_{\mathrm{c} 43}$ & & 31.771247 \\
4 & & $\varepsilon_{\mathrm{c} 43}$ & & 82.790442 \\
5 & & $\sigma_{\mathrm{h} 1}$ & & -25.67215 \\
6 & & $\varepsilon_{\mathrm{h} 1}$ & & 42.493777 \\
1 & 2 & $\sigma_{\mathrm{c} 4}$ & $\varepsilon_{\mathrm{c} 4}$ & 3.6072249 \\
1 & 3 & $\sigma_{\mathrm{c} 4}$ & $\sigma_{\mathrm{c} 43}$ & -7.612901 \\
2 & 3 & $\varepsilon_{\mathrm{c} 4}$ & $\sigma_{\mathrm{c} 43}$ & -14.38961 \\
1 & 4 & $\sigma_{\mathrm{c} 4}$ & $\varepsilon_{\mathrm{c} 43}$ & -14.76551 \\
2 & 4 & $\varepsilon_{\mathrm{c} 4}$ & $\varepsilon_{\mathrm{c} 43}$ & -5.904058 \\
3 & 4 & $\sigma_{\mathrm{c} 43}$ & $\varepsilon_{\mathrm{c} 43}$ & -5.939189 \\
1 & 5 & $\sigma_{\mathrm{c} 4}$ & $\sigma_{\mathrm{h} 1}$ & -10.60018 \\
2 & 5 & $\varepsilon_{\mathrm{c} 4}$ & $\sigma_{\mathrm{h} 1}$ & -7.648735 \\
3 & 5 & $\sigma_{\mathrm{c} 43}$ & $\sigma_{\mathrm{h} 1}$ & 50.824863 \\
4 & 5 & $\varepsilon_{\mathrm{c} 43}$ & $\sigma_{\mathrm{h} 1}$ & -39.13925 \\
1 & 6 & $\sigma_{\mathrm{c} 4}$ & $\varepsilon_{\mathrm{h} 1}$ & -8.379117 \\
2 & 6 & $\varepsilon_{\mathrm{c} 4}$ & $\varepsilon_{\mathrm{h} 1}$ & -10.81184 \\
3 & 6 & $\sigma_{\mathrm{c} 43}$ & $\varepsilon_{\mathrm{h} 1}$ & -25.02224 \\
4 & 6 & $\varepsilon_{\mathrm{c} 43}$ & $\varepsilon_{\mathrm{h} 1}$ & -17.70281 \\
5 & 6 & $\sigma_{\mathrm{h} 1}$ & $\varepsilon_{\mathrm{h} 1}$ & -3.963299 \\
1 & 1 & $\sigma_{\mathrm{c} 4}$ & $\sigma_{\mathrm{c} 4}$ & -23.65182 \\
2 & 2 & $\varepsilon_{\mathrm{c} 4}$ & $\varepsilon_{\mathrm{c} 4}$ & 7.7031104 \\
3 & 3 & $\sigma_{\mathrm{c} 43}$ & $\sigma_{\mathrm{c} 43}$ & -45.9505 \\
4 & 4 & $\varepsilon_{\mathrm{c} 43}$ & $\varepsilon_{\mathrm{c} 43}$ & -9.867059 \\
5 & 5 & $\sigma_{\mathrm{h} 1}$ & $\sigma_{\mathrm{h} 1}$ & -67.95598 \\
6 & 6 & $\varepsilon_{\mathrm{h} 1}$ & $\varepsilon_{\mathrm{h} 1}$ & 11.233854 \\
\hline & & & & \\
\hline
\end{tabular}

$$
\rho_{\text {Norbornane }}=\beta_{0}+\sum_{i=1}^{6} \beta_{i} \bar{x}_{i}+\sum_{i=1}^{6} \sum_{j=1}^{6} \beta_{i j} \bar{x}_{i} \bar{x}_{j}
$$




$$
\bar{x}_{i}=\frac{2\left(x_{i}-x_{i \min }\right)}{x_{i \max }-x_{i \min }}-1
$$

In equation (78), $\beta_{i}$ is the coefficient value found in the fifth column of Table 4.15 and $\bar{x}_{i}$ is the normalized intermolecular multiplier value defined in equation (79). The values $x_{\text {imax }}$ and $x_{\text {imin }}$ in equation (79) are the maximum and minimum settings for the $i^{\text {th }}$ intermolecular multiplier. These maximum and minimum settings are the smallest and largest values for a particular intermolecular multiplier found in Table 4.13.

Table 4.16: Norbornane Specific Heat RSE Coefficients

$$
\begin{array}{ccccc}
\hline i & j & x_{i} & x_{j} & \beta_{i j} \\
\hline 0 & & & & 42.238433 \\
1 & & \sigma_{\mathrm{c} 4} & & -2.324575 \\
2 & & \varepsilon_{\mathrm{c} 4} & -9.091162 \\
3 & & \sigma_{\mathrm{c} 43} & -10.73292 \\
4 & & \varepsilon_{\mathrm{c} 43} & -13.71269 \\
5 & & \sigma_{\mathrm{h} 1} & 12.579713 \\
6 & & \varepsilon_{\mathrm{h} 1} & -14.33476 \\
1 & 1 & \sigma_{\mathrm{c} 4} & \sigma_{\mathrm{c} 4} & 11.67144 \\
6 & 6 & \varepsilon_{\mathrm{h} 1} & \varepsilon_{\mathrm{h} 1} & -23.34893 \\
\hline & & & & \\
C_{P-\text { Norbornane }}= & & & \\
& & & & \\
&
\end{array}
$$

Equation (80) is similar in form to equation (78) except all but two of the second order terms are left out. Only two of the second order terms are kept because they are the second order terms whose changes have the largest impact on $C_{P \text {-Norbornane }}$ (found from a Pareto analysis). 
Table 4.17: Ethyl Azide Density RSE Coefficients

\begin{tabular}{|c|c|c|c|c|}
\hline$i$ & $j$ & $x_{i}$ & $x_{j}$ & $\beta_{i j}$ \\
\hline 0 & & & & 1229.5746 \\
\hline 1 & & $\sigma_{\mathrm{n} 1 \mathrm{z}}$ & & -99.70213 \\
\hline 2 & & $\varepsilon_{\mathrm{n} 1 \mathrm{z}}$ & & 7.0746341 \\
\hline 3 & & $\sigma_{\mathrm{n} 2 \mathrm{t}}$ & & 4.0069674 \\
\hline 4 & & $\varepsilon_{\mathrm{n} 2 \mathrm{t}}$ & & 6.8752419 \\
\hline 5 & & $\sigma_{\mathrm{n} 2 \mathrm{z}}$ & & -7.847345 \\
\hline 6 & & $\varepsilon_{\mathrm{n} 2 \mathrm{z}}$ & & 13.156627 \\
\hline 7 & & $\sigma_{\mathrm{c} 4 \mathrm{z}}$ & & 11.578421 \\
\hline 8 & & $\varepsilon_{\mathrm{c} 4 \mathrm{z}}$ & & 17.775449 \\
\hline 9 & & $\sigma_{\mathrm{h} 1}$ & & -368.9607 \\
\hline 10 & & $\varepsilon_{\mathrm{h} 1}$ & & 31.484708 \\
\hline 1 & 2 & $\sigma_{\mathrm{n} 1 \mathrm{z}}$ & $\varepsilon_{\mathrm{n} 1 \mathrm{z}}$ & 1.6563847 \\
\hline 1 & 3 & $\sigma_{\mathrm{n} 1 \mathrm{z}}$ & $\sigma_{\mathrm{n} 2 \mathrm{t}}$ & 28.97636 \\
\hline 2 & 3 & $\varepsilon_{\mathrm{n} 1 \mathrm{z}}$ & $\sigma_{\mathrm{n} 2 \mathrm{t}}$ & 0.3579746 \\
\hline 1 & 4 & $\sigma_{\mathrm{n} 1 \mathrm{z}}$ & $\varepsilon_{\mathrm{n} 2 \mathrm{t}}$ & 0.4073676 \\
\hline 2 & 4 & $\varepsilon_{\mathrm{n} 1 \mathrm{z}}$ & $\varepsilon_{\mathrm{n} 2 \mathrm{t}}$ & 0.7747843 \\
\hline 3 & 4 & $\sigma_{\mathrm{n} 2 \mathrm{t}}$ & $\varepsilon_{\mathrm{n} 2 \mathrm{t}}$ & 3.2870754 \\
\hline 1 & 5 & $\sigma_{\mathrm{n} 1 \mathrm{z}}$ & $\sigma_{\mathrm{n} 2 \mathrm{z}}$ & 20.290816 \\
\hline 2 & 5 & $\varepsilon_{\mathrm{n} 1 \mathrm{z}}$ & $\sigma_{\mathrm{n} 2 \mathrm{z}}$ & -2.31504 \\
\hline 3 & 5 & $\sigma_{\mathrm{n} 2 \mathrm{t}}$ & $\sigma_{\mathrm{n} 2 \mathrm{z}}$ & 15.873996 \\
\hline 4 & 5 & $\varepsilon_{\mathrm{n} 2 \mathrm{t}}$ & $\sigma_{\mathrm{n} 2 \mathrm{z}}$ & -0.834524 \\
\hline 1 & 6 & $\sigma_{\mathrm{n} 1 \mathrm{z}}$ & $\varepsilon_{\mathrm{n} 2 \mathrm{z}}$ & 1.6962967 \\
\hline 2 & 6 & $\varepsilon_{\mathrm{n} 1 \mathrm{z}}$ & $\varepsilon_{\mathrm{n} 2 \mathrm{z}}$ & 0.866964 \\
\hline 3 & 6 & $\sigma_{\mathrm{n} 2 \mathrm{t}}$ & $\varepsilon_{\mathrm{n} 2 \mathrm{z}}$ & -0.419556 \\
\hline 4 & 6 & $\varepsilon_{n 2 t}$ & $\varepsilon_{\mathrm{n} 2 \mathrm{z}}$ & 0.5934893 \\
\hline 5 & 6 & $\sigma_{\mathrm{n} 2 \mathrm{z}}$ & $\varepsilon_{\mathrm{n} 2 \mathrm{z}}$ & 4.6550237 \\
\hline 1 & 7 & $\sigma_{\mathrm{n} 1 \mathrm{z}}$ & $\sigma_{\mathrm{c} 4 \mathrm{z}}$ & 13.856638 \\
\hline 2 & 7 & $\varepsilon_{\mathrm{n} 1 \mathrm{z}}$ & $\sigma_{c 4 z}$ & 0.5957754 \\
\hline 3 & 7 & $\sigma_{\mathrm{n} 2 \mathrm{t}}$ & $\sigma_{\mathrm{c} 4 \mathrm{z}}$ & 1.0337681 \\
\hline 4 & 7 & $\varepsilon_{\mathrm{n} 2 \mathrm{t}}$ & $\sigma_{\mathrm{c} 4 \mathrm{z}}$ & 1.435135 \\
\hline 5 & 7 & $\sigma_{\mathrm{n} 2 \mathrm{z}}$ & $\sigma_{c 4 z}$ & 8.1702146 \\
\hline 6 & 7 & $\varepsilon_{\mathrm{n} 2 \mathrm{z}}$ & $\sigma_{c 4 z}$ & 0.2808496 \\
\hline 1 & 8 & $\sigma_{\mathrm{n} 1 \mathrm{z}}$ & $\varepsilon_{\mathrm{c} 4 \mathrm{z}}$ & -12.72267 \\
\hline 2 & 8 & $\varepsilon_{\mathrm{n} 1 \mathrm{z}}$ & $\varepsilon_{\mathrm{c} 4 \mathrm{z}}$ & -0.699664 \\
\hline 3 & 8 & $\sigma_{\mathrm{n} 2 \mathrm{t}}$ & $\varepsilon_{\mathrm{c} 4 \mathrm{z}}$ & -4.961784 \\
\hline 4 & 8 & $\varepsilon_{n 2 t}$ & $\varepsilon_{\mathrm{c} 4 \mathrm{z}}$ & -0.748892 \\
\hline 5 & 8 & $\sigma_{\mathrm{n} 2 \mathrm{z}}$ & $\varepsilon_{\mathrm{c} 4 \mathrm{z}}$ & -12.77332 \\
\hline 6 & 8 & $\varepsilon_{\mathrm{n} 2 \mathrm{z}}$ & $\varepsilon_{\mathrm{c} 4 \mathrm{z}}$ & 7.7261451 \\
\hline 7 & 8 & $\sigma_{\mathrm{c} 4 \mathrm{z}}$ & $\varepsilon_{\mathrm{c} 4 \mathrm{z}}$ & 25.028974 \\
\hline 1 & 1 & $\sigma_{\mathrm{n} 1 \mathrm{z}}$ & $\sigma_{\mathrm{n} 1 \mathrm{z}}$ & -33.56724 \\
\hline 2 & 2 & $\varepsilon_{\mathrm{n} 1 \mathrm{z}}$ & $\varepsilon_{\mathrm{n} 1 \mathrm{z}}$ & -3.919436 \\
\hline 3 & 3 & $\sigma_{\mathrm{n} 2 \mathrm{t}}$ & $\sigma_{\mathrm{n} 2 \mathrm{t}}$ & -19.6184 \\
\hline 4 & 4 & $\varepsilon_{n 2 t}$ & $\varepsilon_{\mathrm{n} 2 \mathrm{t}}$ & 4.1803872 \\
\hline 5 & 5 & $\sigma_{\mathrm{n} 2 \mathrm{z}}$ & $\sigma_{\mathrm{n} 2 \mathrm{z}}$ & -26.17988 \\
\hline 6 & 6 & $\varepsilon_{\mathrm{n} 2 \mathrm{z}}$ & $\varepsilon_{\mathrm{n} 2 \mathrm{z}}$ & 4.7082149 \\
\hline 7 & 7 & $\sigma_{\mathrm{c} 4 \mathrm{z}}$ & $\sigma_{\mathrm{c} 4 \mathrm{z}}$ & -34.9253 \\
\hline 8 & 8 & $\varepsilon_{\mathrm{c} 4 \mathrm{z}}$ & $\varepsilon_{\mathrm{c} 4 \mathrm{z}}$ & -2.513984 \\
\hline 9 & 1 & $\sigma_{\mathrm{h} 1}-1.00135$ & $\sigma_{\mathrm{n} 1 \mathrm{z}}$ & 156.59829 \\
\hline 9 & 2 & $\sigma_{\mathrm{h} 1}-1.00135$ & $\varepsilon_{\mathrm{n} 1 \mathrm{z}}$ & -3.604505 \\
\hline
\end{tabular}


Table 4.17 (continued)

\begin{tabular}{ccccc}
\hline 9 & 3 & $\sigma_{\mathrm{h} 1}-1.00135$ & $\sigma_{\mathrm{n} 2 \mathrm{t}}$ & 16.333168 \\
9 & 4 & $\sigma_{\mathrm{h} 1}-1.00135$ & $\varepsilon_{\mathrm{n} 2 \mathrm{t}}$ & -12.24265 \\
9 & 5 & $\sigma_{\mathrm{h} 1}-1.00135$ & $\sigma_{\mathrm{n} 2 \mathrm{z}}$ & 74.35384 \\
9 & 6 & $\sigma_{\mathrm{h} 1}-1.00135$ & $\varepsilon_{\mathrm{n} 2 \mathrm{z}}$ & -32.39317 \\
9 & 7 & $\sigma_{\mathrm{h} 1}-1.00135$ & $\sigma_{\mathrm{c} 4 \mathrm{z}}$ & 59.161797 \\
9 & 8 & $\sigma_{\mathrm{h} 1}-1.00135$ & $\varepsilon_{\mathrm{c} 4 \mathrm{z}}$ & 42.71738 \\
9 & 10 & $\sigma_{\mathrm{h} 1}-1.00135$ & $\varepsilon_{\mathrm{h} 1}-1.00405$ & 0 \\
10 & 1 & $\varepsilon_{\mathrm{h} 1}-1.00405$ & $\sigma_{\mathrm{n} 1 \mathrm{z}}$ & -10.02733 \\
10 & 2 & $\varepsilon_{\mathrm{h} 1}-1.00405$ & $\varepsilon_{\mathrm{n} 1 \mathrm{z}}$ & 0.0819719 \\
10 & 3 & $\varepsilon_{\mathrm{h} 1}-1.00405$ & $\sigma_{\mathrm{n} 2 \mathrm{t}}$ & -17.85526 \\
10 & 4 & $\varepsilon_{\mathrm{h} 1}-1.00405$ & $\varepsilon_{\mathrm{n} 2 \mathrm{t}}$ & -3.982509 \\
10 & 5 & $\varepsilon_{\mathrm{h} 1}-1.00405$ & $\sigma_{\mathrm{n} 2 \mathrm{z}}$ & -14.00624 \\
10 & 6 & $\varepsilon_{\mathrm{h} 1}-1.00405$ & $\varepsilon_{\mathrm{n} 2 \mathrm{z}}$ & 0 \\
10 & 7 & $\varepsilon_{\mathrm{h} 1}-1.00405$ & $\sigma_{\mathrm{c} 4 \mathrm{z}}$ & 0 \\
10 & 8 & $\varepsilon_{\mathrm{h} 1}-1.00405$ & $\varepsilon_{\mathrm{c} 4 \mathrm{z}}$ & -10.95187 \\
9 & 9 & $\sigma_{\mathrm{h} 1}-1.00135$ & $\sigma_{\mathrm{h} 1}-1.00135$ & -900.4714 \\
10 & 10 & $\varepsilon_{\mathrm{h} 1}-1.00405$ & $\varepsilon_{\mathrm{h} 1}-1.00405$ & 10.100483 \\
\hline
\end{tabular}

The RSE for the density (and specific heat) of ethyl azide differs from that for norbornane because of a limitation in the software used to create the RSE (JMP). JMP limits the number of design variables that can be used in a response surface analysis to 8 [57]. In order to get around this limitation, any additional design variables (in this case, $\sigma_{\mathrm{h} 1}$ and $\left.\varepsilon_{\mathrm{h} 1}\right)$ are not normalized by the design variable normalization equation, equation (79).

$$
\begin{aligned}
\rho_{\text {Ethyl Azide }}= & \beta_{0}+\sum_{i=1}^{8} \beta_{i} \bar{x}_{i}+\sum_{i=9}^{10} \beta_{i} x_{i}+\sum_{i=1}^{8} \sum_{j=1}^{8} \beta_{i j} \bar{x}_{i} \bar{x}_{j}+ \\
& \sum_{i=9}^{10} \sum_{j=1}^{8} \beta_{i j}\left(x_{i}-x_{i 0}\right) \bar{x}_{j}+\sum_{i=9}^{10} \sum_{j=9}^{10} \beta_{i j}\left(x_{i}-x_{i 0}\right)\left(x_{j}-x_{j 0}\right)
\end{aligned}
$$

In equation (81), $\bar{x}_{i}$ is the normalized intermolecular multiplier value defined in equation (79) while $x_{i}$ is non-normalized intermolecular multiplier value. The terms $x_{i 0}$ and $x_{j 0}$ are the values shown subtracted from the $\sigma_{\mathrm{h} 1}$ and $\varepsilon_{\mathrm{h} 1}$ intermolecular multiplier values in the second order terms found in the third and fourth columns of Table 4.17. 
The summations in equation (81) are broken up into normalized (1-8) and nonnormalized (9-10) terms.

Table 4.18: Ethyl Azide Specific Heat RSE Coefficients

\begin{tabular}{ccccc}
\hline$i$ & $j$ & $x_{i}$ & $x_{j}$ & $\beta_{i j}$ \\
\hline 0 & & & 47.361169 \\
1 & & $\sigma_{\mathrm{n} 1 \mathrm{z}}$ & -1.97035 \\
2 & & $\varepsilon_{\mathrm{n} 1 \mathrm{z}}$ & -0.14695 \\
3 & & $\sigma_{\mathrm{n} 2 \mathrm{t}}$ & 0.3973 \\
4 & $\varepsilon_{\mathrm{n} 2 \mathrm{t}}$ & 2.03885 \\
5 & $\sigma_{\mathrm{n} 2 \mathrm{z}}$ & -3.7973 \\
6 & $\varepsilon_{\mathrm{n} 2 \mathrm{z}}$ & -1.06255 \\
7 & $\sigma_{\mathrm{c} 4 \mathrm{z}}$ & -1.083 \\
8 & $\varepsilon_{\mathrm{c} 4 \mathrm{z}}$ & 0.3715 \\
9 & $\sigma_{\mathrm{h} 1}$ & -12.578 \\
10 & $\varepsilon_{\mathrm{h} 1}$ & 0.498 \\
1 & $\sigma_{\mathrm{n} 1 \mathrm{z}}$ & $\sigma_{\mathrm{n} 1 \mathrm{z}}$ & -0.808719 \\
4 & 4 & $\varepsilon_{\mathrm{n} 2 \mathrm{t}}$ & $\varepsilon_{\mathrm{n} 2 \mathrm{t}}$ & -0.878019 \\
5 & 5 & $\sigma_{\mathrm{n} 2 \mathrm{z}}$ & $\sigma_{\mathrm{n} 2 \mathrm{z}}$ & -1.025469 \\
9 & 9 & $\sigma_{\mathrm{h} 1}-1$ & $\sigma_{\mathrm{h} 1}-1$ & -5.769231 \\
\hline \multicolumn{5}{c}{} \\
$C_{P-\text { Ethyl Azide }}=\beta_{0}+\sum_{i=1}^{8} \beta_{i} \bar{x}_{i}+\sum_{i=9}^{10} \beta_{i} x_{i}+\beta_{11} \bar{x}_{1} \bar{x}_{1}+\beta_{44} \bar{x}_{4} \bar{x}_{4}$
\end{tabular}

Equation (82) has a similar structure to equation (81) except only the most influential second order terms (based upon a Pareto analysis) are kept in the RSE. This is due to the same reasoning described previously for norbornane: it is believed the specific heat design space is smoother (there are no large jumps in specific heat from run to run like there are with density).

Table 4.19 provides the fit statistics for the four RSE's created. Each RSE predicts the molecular dynamics results fairly accurately with the largest RMS error being $2.48 \%$. 
Table 4.19: RSE Fit Statistics

\begin{tabular}{ccc}
\hline & $R^{2}$ & $R M S$ Error \\
\hline Norbornane Density & 0.9885 & $1.19 \%$ \\
Norbornane Specific Heat & 0.9688 & $1.34 \%$ \\
Ethyl Azide Density & 0.9625 & $2.48 \%$ \\
Ethyl Azide Specific Heat & 0.9078 & $1.12 \%$ \\
\hline
\end{tabular}

\section{Optimization and Validation}

With the RSE's created, the settings for the intermolecular parameters can be optimized in order to best predict the experimental values for density and specific heat at the temperature and pressure used in the DOE runs. The goal of the optimization is to minimize the change in the intermolecular parameters from the baseline COMPASS values subject to the constraint that the optimized solutions (predicted weighted normalized density and specific heat) have a specified maximum error with respect to the molecular dynamics predictions for these values. This optimization form is used in order to avoid large changes to the baseline COMPASS model values while still satisfying the constraint that the predicted values from the RSE must reasonably match the molecular dynamics results.

Equations (25) and (26), repeated in equations (83) and (84) for convenience, describe this optimization method in detail.

$$
\begin{gathered}
S=\sum_{i=1}^{N}\left(x_{i}-1\right)^{2} \\
g=\sum_{i=1}^{M}\left[W_{\rho_{-} i} \sqrt{\left(\frac{\rho_{\text {predicted }_{-} i}-\rho_{\text {actual }_{-} i}}{\rho_{\text {actual }} i}\right)^{2}}+W_{c p_{-} i} \sqrt{\left(\frac{C_{P_{\text {predicted } \_}-}-C_{P_{\text {actual }_{-} i}}}{C_{P_{\text {actual }} i}}\right)^{2}}\right] \\
-E_{\max } \leq 0
\end{gathered}
$$


The goal of the optimization is to minimize the objective function $S$, subject to the constraint $g$ less than or equal to zero. The value $x_{i}$ in equation (83) is the intermolecular parameter multiplier for atom type $i$. The summation in equation (83) is over the number of atom type intermolecular parameters changed for a given compound. The parameters $\rho_{\text {actual_ } i}$ and $C_{\text {Pactual_i }}$ in equation (84) are the experimental values for density and specific heat respectively at the $i^{\text {th }}$ temperature and pressure. The parameters $\rho_{\text {predicted } \_i}$ and $C_{\text {Ppredicted_i }}$ are the density and specific heat predicted by the RSE's at the same temperature and pressure. The parameters $W_{\rho_{-} i}$ and $W_{c p_{-} i}$ in equation (84) are the weighting values for density and specific heat respectively at the same temperature and pressure. Only one temperature and pressure point is used for both norbornane and ethyl azide due to a limited amount of experimental data for these model compounds. The weighting values were each set to 0.5 based upon the results from the engine level sensitivity study provided in section 1.2.2. These results showed a nearly equal sensitivity of specific impulse to changes in density, enthalpy, or entropy. $E_{\max }$, the maximum allowable weighted error, is set to 0.01 . This means that the sum of the two weighted deviations between the RSE predicted value and the experimental value must be less than $1.0 \%$. This value was chosen in order to obtain a fairly close approximation of experimental results at the temperature and pressure of interest. Table 4.20 and Table 4.21 are the results of this optimization for norbornane and ethyl azide respectively.

Table 4.20: Norbornane Optimized Intermolecular Parameters

\begin{tabular}{cccl}
\hline $\begin{array}{c}\text { Intermolecular } \\
\text { Parameter }\end{array}$ & $x_{i}$ & $\begin{array}{c}\text { Baseline COMPASS } \\
\text { Value }\end{array}$ & Optimized Value \\
\hline$\sigma_{\mathrm{c} 4}$ & 1.0899 & $3.85400 \AA$ & $4.20031 \AA$ \\
$\varepsilon_{\mathrm{c} 4}$ & 1.4685 & $0.06200 \mathrm{kcal} / \mathrm{mol}$ & $0.09105 \mathrm{kcal} / \mathrm{mol}$ \\
$\sigma_{\mathrm{c} 43}$ & 0.9448 & $3.85400 \AA$ & $3.64123 \AA$ \\
$\varepsilon_{\mathrm{c} 43}$ & 1.4332 & $0.04000 \mathrm{kcal} / \mathrm{mol}$ & $0.05733 \mathrm{kcal} / \mathrm{mol}$ \\
$\sigma_{\mathrm{h} 1}$ & 0.7974 & $2.87800 \AA$ & $2.29484 \AA$ \\
$\varepsilon_{\mathrm{h} 1}$ & 1.1797 & $0.02300 \mathrm{kcal} / \mathrm{mol}$ & $0.02713 \mathrm{kcal} / \mathrm{mol}$ \\
\hline
\end{tabular}


Table 4.21: Ethyl Azide Optimized Intermolecular Parameters

\begin{tabular}{ccll}
\hline $\begin{array}{c}\text { Intermolecular } \\
\text { Parameter }\end{array}$ & $x_{i}$ & $\begin{array}{c}\text { Baseline COMPASS } \\
\text { Value }\end{array}$ & Optimized Value \\
\hline$\sigma_{\mathrm{n} 1 \mathrm{z}}$ & 0.9822 & $3.52000 \AA$ & $3.45717 \AA$ \\
$\varepsilon_{\mathrm{n} 1 \mathrm{z}}$ & 1.0125 & $0.08500 \mathrm{kcal} / \mathrm{mol}$ & $0.08606 \mathrm{kcal} / \mathrm{mol}$ \\
$\sigma_{\mathrm{n} 2 \mathrm{t}}$ & 1.0008 & $3.30000 \AA$ & $3.30264 \AA$ \\
$\varepsilon_{\mathrm{n} 2 \mathrm{t}}$ & 0.8542 & $0.05000 \mathrm{kcal} / \mathrm{mol}$ & $0.04271 \mathrm{kcal} / \mathrm{mol}$ \\
$\sigma_{\mathrm{n} 2 \mathrm{z}}$ & 1.1757 & $3.40000 \AA$ & $3.99723 \AA$ \\
$\varepsilon_{\mathrm{n} 2 \mathrm{z}}$ & 1.0753 & $0.12000 \mathrm{kcal} / \mathrm{mol}$ & $0.12903 \mathrm{kcal} / \mathrm{mol}$ \\
$\sigma_{\mathrm{c} 4 \mathrm{z}}$ & 1.0497 & $3.65000 \AA$ & $3.83136 \AA$ \\
$\varepsilon_{\mathrm{c} 4 \mathrm{z}}$ & 1.0087 & $0.08000 \mathrm{kcal} / \mathrm{mol}$ & $0.08070 \mathrm{kcal} / \mathrm{mol}$ \\
$\sigma_{\mathrm{h} 1}$ & 1.0184 & $2.87800 \AA$ & $2.93090 \AA$ \\
$\varepsilon_{\mathrm{h} 1}$ & 1.0019 & $0.02300 \mathrm{kcal} / \mathrm{mol}$ & $0.02304 \mathrm{kcal} / \mathrm{mol}$ \\
\hline
\end{tabular}

Molecular dynamics validation simulations were performed using these optimized intermolecular parameters to verify that the RSE predicted values for density and specific heat matched the molecular dynamics results (Table 4.22).

Table 4.22: Molecular Dynamics Validation Results

\begin{tabular}{cccc}
\hline & RSE Prediction & MD Validation & Experimental \\
\hline Norbornane $-360.00 \mathrm{~K}, 1$ & $\mathrm{~atm}$ & & \\
Density $\left(\mathrm{kg} / \mathrm{m}^{3}\right)$ & 895.72 & 851.45 & 914.00 \\
$\mathrm{C}_{\mathrm{P}}(\mathrm{cal} / \mathrm{mol}-\mathrm{K})$ & 36.07 & 39.59 & 36.07 \\
\hline Ethyl Azide $-298.15 \mathrm{~K}, 1 \mathrm{~atm}$ & & & \\
Density $\left(\mathrm{kg} / \mathrm{m}^{3}\right)$ & 876.47 & 864.56 & 876.50 \\
$\mathrm{C}_{\mathrm{P}}(\mathrm{cal} / \mathrm{mol}-\mathrm{K})$ & 29.25 & 30.96 & 28.00 \\
\hline
\end{tabular}

The norbornane RSE prediction of density is $5.2 \%$ higher than the molecular dynamics validation. The norbornane RSE prediction of specific heat is $8.9 \%$ lower than the molecular dynamics validation. The ethyl azide RSE prediction of density is $1.1 \%$ higher than the molecular dynamics validation. The ethyl azide RSE prediction of specific heat is $8.0 \%$ lower than the molecular dynamics validation. These RSE property 
predictions are sufficiently accurate with the density prediction errors under $5.2 \%$ and the specific heat prediction errors under $9 \%$.

The acceptable maximum error in specific heat prediction is higher than that for density, because specific heat is used in only one of two components of the calculation of the total enthalpy and total entropy (the other being the formation enthalpy and entropy found from either literature or quantum mechanical calculations). The calculation for total enthalpy is provided again in equation (85). The specific heat is used in the calculation of the second two terms on the right hand side of equation (85).

$$
h(T, P)=\Delta h_{f}^{0}+h_{\text {sens }}(T)=\Delta h_{f}^{0}+h_{\text {sens }}^{i g}(T)+h_{\text {sens }}^{\text {res }}(T, P)
$$

The heat of formation of liquid norbornane at $298.15 \mathrm{~K}$ and 1 atm is -22.0 $\mathrm{kcal} / \mathrm{mol}$ [77]. Assuming a constant $\mathrm{C}_{\mathrm{P}}$, the sensible enthalpy of norbornane from 298.15 $\mathrm{K}$ to $380 \mathrm{~K}$ (both at $1 \mathrm{~atm}$ ) is predicted to be $2.95 \mathrm{kcal} / \mathrm{mol}$ from the RSE prediction and $3.24 \mathrm{kcal} / \mathrm{mol}$ from the molecular dynamics simulations. This results in a total enthalpy at $380 \mathrm{~K}$ of $-19.05 \mathrm{kcal} / \mathrm{mol}$ from the RSE prediction and $-18.76 \mathrm{kcal} / \mathrm{mol}$ from the molecular dynamics simulations. This error in total enthalpy prediction by the RSE is only $1.54 \%$, significantly lower than the errors found in the prediction of specific heat. It is this total enthalpy that is needed by rocket engine powerhead design codes.

\subsection{HEDM COMPOUNDS: QUADRICYCLANE AND DMAZ}

With the optimized values for the intermolecular parameters validated, these values can then be used in the prediction of the thermophysical properties of each model compound's corresponding HEDM compound. 


\subsubsection{QUANTUM MECHANICS}

Like the model compounds, a quantum mechanical analysis must first be completed. This quantum mechanical analysis is performed to optimize the molecular configuration of the HEDM compound and determine the vibrational normal mode frequencies to predict the ideal gas sensible enthalpy.

Using the same process described for the model compounds in section 4.1.1, the following tables provide molecular geometries (Table 4.23 and Table 4.24), normal mode frequencies (Table 4.25 and Table 4.26), and ideal gas sensible enthalpies (Table 4.27 and Table 4.28) for quadricyclane and DMAZ: 
Table 4.23: Atomic Coordinates for Optimized Quadricyclane Geometry

\begin{tabular}{ccccccc}
\hline & Conformational Search Results & \multicolumn{3}{c}{ GAMESS Results } \\
\hline Atom & $X(\AA)$ & $Y(\AA)$ & $Z(\AA)$ & $X(\AA)$ & $Y(\AA)$ & $Z(\AA)$ \\
\hline C & 0.080 & 0.060 & 2.350 & 0.078 & 0.060 & 2.370 \\
C & 0.044 & -1.103 & 1.370 & 0.046 & -1.086 & 1.373 \\
C & 0.191 & 1.232 & 1.387 & 0.188 & 1.215 & 1.390 \\
C & -0.648 & -0.654 & 0.086 & -0.635 & -0.654 & 0.085 \\
C & 0.888 & -0.751 & 0.147 & 0.875 & -0.752 & 0.145 \\
C & 0.986 & 0.795 & 0.159 & 0.973 & 0.796 & 0.157 \\
C & -0.551 & 0.893 & 0.097 & -0.537 & 0.893 & 0.096 \\
H & 0.962 & -0.001 & 3.030 & 0.943 & 0.003 & 3.044 \\
H & -0.853 & 0.114 & 2.960 & -0.835 & 0.110 & 2.977 \\
H & -0.036 & -2.124 & 1.756 & -0.034 & -2.122 & 1.694 \\
H & 0.239 & 2.249 & 1.788 & 0.242 & 2.247 & 1.727 \\
H & -1.381 & -1.211 & -0.488 & -1.347 & -1.259 & -0.467 \\
H & 1.589 & -1.399 & -0.368 & 1.547 & -1.443 & -0.353 \\
H & 1.763 & 1.357 & -0.347 & 1.726 & 1.405 & -0.330 \\
H & -1.208 & 1.545 & -0.466 & -1.167 & 1.589 & -0.448 \\
\hline
\end{tabular}

Table 4.24: Atomic Coordinates for Optimized DMAZ Geometry

\begin{tabular}{ccccccc}
\hline & \multicolumn{3}{c}{ Conformational Search Results } & \multicolumn{3}{c}{ GAMESS Results } \\
\hline Atom & $X(\AA)$ & $Y(\AA)$ & $Z(\AA)$ & $X(\AA)$ & $Y(\AA)$ & $Z(\AA)$ \\
\hline $\mathrm{N}$ & -1.706 & 0.481 & -3.595 & -1.753 & 0.567 & -3.574 \\
$\mathrm{~N}$ & -1.480 & -0.240 & -2.794 & -1.459 & -0.240 & -2.820 \\
$\mathrm{~N}$ & -1.355 & -1.058 & -1.886 & -1.326 & -1.152 & -1.996 \\
$\mathrm{C}$ & 0.012 & -1.562 & -1.708 & 0.053 & -1.595 & -1.713 \\
$\mathrm{H}$ & 0.485 & -1.751 & -2.667 & 0.596 & -1.791 & -2.648 \\
$\mathrm{H}$ & -0.094 & -2.515 & -1.206 & -0.053 & -2.550 & -1.191 \\
$\mathrm{C}$ & 0.855 & -0.631 & -0.842 & 0.819 & -0.612 & -0.820 \\
$\mathrm{H}$ & 1.782 & -1.138 & -0.558 & 1.749 & -1.100 & -0.465 \\
$\mathrm{~N}$ & 1.107 & 0.642 & -1.494 & 1.078 & 0.660 & -1.489 \\
$\mathrm{C}$ & 2.241 & 0.604 & -2.393 & 2.203 & 0.594 & -2.413 \\
$\mathrm{H}$ & 2.130 & -0.196 & -3.115 & 2.046 & -0.193 & -3.157 \\
$\mathrm{H}$ & 3.191 & 0.456 & -1.871 & 3.168 & 0.395 & -1.906 \\
$\mathrm{H}$ & 2.301 & 1.535 & -2.944 & 2.289 & 1.544 & -2.952 \\
$\mathrm{C}$ & 1.198 & 1.750 & -0.566 & 1.226 & 1.765 & -0.550 \\
$\mathrm{H}$ & 0.286 & 1.821 & 0.014 & 0.327 & 1.848 & 0.069 \\
$\mathrm{H}$ & 1.314 & 2.676 & -1.117 & 1.342 & 2.703 & -1.105 \\
$\mathrm{H}$ & 2.040 & 1.663 & 0.127 & 2.101 & 1.659 & 0.122 \\
$\mathrm{H}$ & 0.299 & -0.451 & 0.069 & 0.202 & -0.417 & 0.063 \\
\hline
\end{tabular}


Table 4.25: Normal Mode Vibrational Frequencies of Quadricyclane

\begin{tabular}{|c|c|c|c|}
\hline Mode & $\begin{array}{l}\text { Wave \#, } k \\
\text { (1/cm) }\end{array}$ & $\theta_{v i b}(K)$ & $\begin{array}{c}C_{V}^{v i b}-298.15 \mathrm{~K} \\
(\mathrm{~J} / \mathrm{mol}-\mathrm{K})\end{array}$ \\
\hline 1 & 321 & 462 & 6.8328 \\
\hline 2 & 458 & 659 & 5.6193 \\
\hline 3 & 590 & 848 & 4.4085 \\
\hline 4 & 638 & 917 & 3.9874 \\
\hline 5 & 642 & 923 & 3.9535 \\
\hline 6 & 660 & 949 & 3.7998 \\
\hline 7 & 689 & 991 & 3.5607 \\
\hline 8 & 713 & 1026 & 3.3627 \\
\hline 9 & 751 & 1080 & 3.0753 \\
\hline 10 & 765 & 1101 & 2.9704 \\
\hline 11 & 818 & 1177 & 2.5981 \\
\hline 12 & 821 & 1181 & 2.5832 \\
\hline 13 & 831 & 1195 & 2.5176 \\
\hline 14 & 862 & 1241 & 2.3148 \\
\hline 15 & 883 & 1271 & 2.1888 \\
\hline 16 & 897 & 1291 & 2.1096 \\
\hline 17 & 900 & 1294 & 2.0946 \\
\hline 18 & 905 & 1302 & 2.0641 \\
\hline 19 & 933 & 1342 & 1.9116 \\
\hline 20 & 950 & 1367 & 1.8211 \\
\hline 21 & 954 & 1373 & 1.8002 \\
\hline 22 & 1003 & 1444 & 1.5625 \\
\hline 23 & 1052 & 1514 & 1.3542 \\
\hline 24 & 1075 & 1547 & 1.2621 \\
\hline 25 & 1107 & 1592 & 1.1474 \\
\hline 26 & 1137 & 1636 & 1.0456 \\
\hline 27 & 1150 & 1655 & 1.0034 \\
\hline 28 & 1163 & 1673 & 0.9648 \\
\hline 29 & 1243 & 1789 & 0.7458 \\
\hline 30 & 1272 & 1830 & 0.6787 \\
\hline 31 & 1352 & 1945 & 0.5205 \\
\hline 32 & 2799 & 4026 & 0.0021 \\
\hline 33 & 2842 & 4088 & 0.0017 \\
\hline 34 & 2915 & 4194 & 0.0013 \\
\hline 35 & 2939 & 4228 & 0.0012 \\
\hline 36 & 2945 & 4238 & 0.0011 \\
\hline 37 & 2951 & 4246 & 0.0011 \\
\hline 38 & 2952 & 4247 & 0.0011 \\
\hline 39 & 2964 & 4264 & 0.0010 \\
\hline
\end{tabular}


Table 4.26: Normal Mode Vibrational Frequencies of DMAZ

\begin{tabular}{|c|c|c|c|}
\hline Mode & $\begin{array}{c}\text { Wave \#, } k \\
\text { (1/cm) }\end{array}$ & $\theta_{v i b}(K)$ & $\begin{array}{c}C_{V}^{v i b}-298.15 \mathrm{~K} \\
(\mathrm{~J} / \mathrm{mol}-\mathrm{K})\end{array}$ \\
\hline 1 & 48 & 69 & 8.2772 \\
\hline 2 & 62 & 89 & 8.2526 \\
\hline 3 & 99 & 142 & 8.1579 \\
\hline 4 & 217 & 312 & 7.5944 \\
\hline 5 & 233 & 335 & 7.4911 \\
\hline 6 & 251 & 361 & 7.3682 \\
\hline 7 & 288 & 414 & 7.0960 \\
\hline 8 & 342 & 492 & 6.6588 \\
\hline 9 & 407 & 586 & 6.0876 \\
\hline 10 & 428 & 616 & 5.8963 \\
\hline 11 & 522 & 751 & 5.0260 \\
\hline 12 & 557 & 801 & 4.7043 \\
\hline 13 & 651 & 937 & 3.8737 \\
\hline 14 & 782 & 1125 & 2.8487 \\
\hline 15 & 849 & 1222 & 2.3989 \\
\hline 16 & 962 & 1384 & 1.7602 \\
\hline 17 & 985 & 1417 & 1.6482 \\
\hline 18 & 1057 & 1521 & 1.3340 \\
\hline 19 & 1067 & 1535 & 1.2946 \\
\hline 20 & 1073 & 1544 & 1.2714 \\
\hline 21 & 1116 & 1606 & 1.1153 \\
\hline 22 & 1171 & 1685 & 0.9396 \\
\hline 23 & 1206 & 1735 & 0.8408 \\
\hline 24 & 1272 & 1830 & 0.6791 \\
\hline 25 & 1304 & 1876 & 0.6112 \\
\hline 26 & 1315 & 1892 & 0.5893 \\
\hline 27 & 1342 & 1931 & 0.5386 \\
\hline 28 & 1384 & 1991 & 0.4675 \\
\hline 29 & 1405 & 2021 & 0.4352 \\
\hline 30 & 1443 & 2076 & 0.3820 \\
\hline 31 & 1466 & 2109 & 0.3528 \\
\hline 32 & 1485 & 2137 & 0.3302 \\
\hline 33 & 1486 & 2138 & 0.3291 \\
\hline 34 & 1497 & 2154 & 0.3167 \\
\hline 35 & 1500 & 2158 & 0.3134 \\
\hline 36 & 1501 & 2160 & 0.3123 \\
\hline 37 & 1512 & 2175 & 0.3005 \\
\hline 38 & 2236 & 3217 & 0.0199 \\
\hline 39 & 2896 & 4167 & 0.0014 \\
\hline 40 & 2910 & 4187 & 0.0013 \\
\hline 41 & 2920 & 4201 & 0.0013 \\
\hline 42 & 3025 & 4352 & 0.0008 \\
\hline 43 & 3059 & 4401 & 0.0007 \\
\hline 44 & 3065 & 4410 & 0.0007 \\
\hline 45 & 3073 & 4421 & 0.0007 \\
\hline 46 & 3101 & 4462 & 0.0006 \\
\hline 47 & 3103 & 4465 & 0.0006 \\
\hline 48 & 3105 & 4467 & 0.0006 \\
\hline
\end{tabular}


Table 4.27: Ideal Gas Sensible Enthalpy Results for Quadricyclane

\begin{tabular}{cccccc}
\hline $\begin{array}{c}\text { Temperature } \\
(K)\end{array}$ & $\begin{array}{c}C_{V_{-} \text {trans }} \\
(\mathrm{J} / \mathrm{mol}-\mathrm{K})\end{array}$ & $\begin{array}{c}C_{V_{-} \text {rot }} \\
(\mathrm{J} / \mathrm{mol}-\mathrm{K})\end{array}$ & $\begin{array}{c}C_{V_{-} \text {vib }} \\
(\mathrm{J} / \mathrm{mol}-\mathrm{K})\end{array}$ & $\begin{array}{c}C_{V} \\
(\mathrm{~J} / \mathrm{mol}-\mathrm{K})\end{array}$ & $\begin{array}{c}h_{\text {sens }}^{\text {ig }}-R T \\
(\mathrm{~kJ} / \mathrm{mol})\end{array}$ \\
\hline 283.00 & 12.4715 & 12.4715 & 68.5056 & 93.4485 & -1.4716 \\
293.00 & 12.4715 & 12.4715 & 73.3760 & 98.3189 & -0.5128 \\
298.15 & 12.4715 & 12.4715 & 75.8699 & 100.8128 & 0.0000 \\
303.00 & 12.4715 & 12.4715 & 78.2070 & 103.1499 & 0.4946 \\
313.00 & 12.4715 & 12.4715 & 82.9839 & 107.9268 & 1.5500 \\
323.00 & 12.4715 & 12.4715 & 87.6941 & 112.6370 & 2.6528 \\
333.00 & 12.4715 & 12.4715 & 92.3272 & 117.2701 & 3.8023 \\
343.00 & 12.4715 & 12.4715 & 96.8750 & 121.8179 & 4.9978 \\
\hline
\end{tabular}

Table 4.28: Ideal Gas Sensible Enthalpy Results for DMAZ

\begin{tabular}{cccccc}
\hline $\begin{array}{c}\text { Temperature } \\
(K)\end{array}$ & $\begin{array}{c}C_{V_{-} \text {trans }} \\
(\mathrm{J} / \text { mol-K) }\end{array}$ & $\begin{array}{c}C_{V_{-} \text {rot }} \\
(\mathrm{J} / \mathrm{mol}-\mathrm{K})\end{array}$ & $\begin{array}{c}C_{V_{-} \text {vib }} \\
(\mathrm{J} / \mathrm{mol}-\mathrm{K})\end{array}$ & $\begin{array}{c}C_{V} \\
(\mathrm{~J} / \mathrm{mol}-\mathrm{K})\end{array}$ & $\begin{array}{c}h_{\text {sens }}^{\text {ig }}-R T \\
(\mathrm{~kJ} / \mathrm{mol})\end{array}$ \\
\hline 293.25 & 12.4715 & 12.4715 & 106.1922 & 131.1351 & -0.6468 \\
298.15 & 12.4715 & 12.4715 & 107.9224 & 132.8653 & 0.0000 \\
313.00 & 12.4715 & 12.4715 & 113.1925 & 138.1354 & 2.0122 \\
333.10 & 12.4715 & 12.4715 & 120.3644 & 145.3073 & 4.8608 \\
353.05 & 12.4715 & 12.4715 & 127.4865 & 152.4294 & 7.8307 \\
368.05 & 12.4715 & 12.4715 & 132.8183 & 157.7612 & 10.1571 \\
387.35 & 12.4715 & 12.4715 & 139.6201 & 164.5630 & 13.2676 \\
404.55 & 12.4715 & 12.4715 & 145.6038 & 170.5467 & 16.1495 \\
418.55 & 12.4715 & 12.4715 & 150.4070 & 175.3499 & 18.5708 \\
435.95 & 12.4715 & 12.4715 & 156.2798 & 181.2227 & 21.6730 \\
\hline
\end{tabular}

\subsubsection{MOLECULAR DYNAMICS}

Using the validated optimized intermolecular parameters from the model compounds and the calculated ideal gas sensible enthalpy for the HEDM compounds, density, enthalpy, and entropy predictions can be made for the HEDM compounds.

\section{Comparison with Experimental Data}

Molecular dynamics simulations of the HEDM compounds are first performed at temperature and pressure combinations for which experimental data exists. These runs are performed to verify the accuracy of the molecular dynamics predictions of density, 
enthalpy, and entropy using the optimized intermolecular parameters obtained from analysis of each HEDM compound's corresponding model compound. The results of these molecular dynamics simulations are provided in Figure 4.3 - Figure 4.8. Both the baseline COMPASS model simulations (those using the published baseline COMPASS intermolecular parameter values) and the optimized intermolecular parameter simulations (using the optimized intermolecular parameters as a result of this thesis research) are provided. As a result of this thesis research, significant improvements in the predictive accuracy of molecular dynamics simulations are achieved.

The experimental measurements and corresponding molecular dynamics simulations for DMAZ are performed at a range of pressures. The experimental measurements of DMAZ at the three highest temperatures were performed at elevated pressures in order to suppress boiling. Minimum and maximum bars are provided for the DMAZ experimental measurements. A published data point [3] for the density of DMAZ at $298 \mathrm{~K}$ is provided in Figure 4.6 for reference.

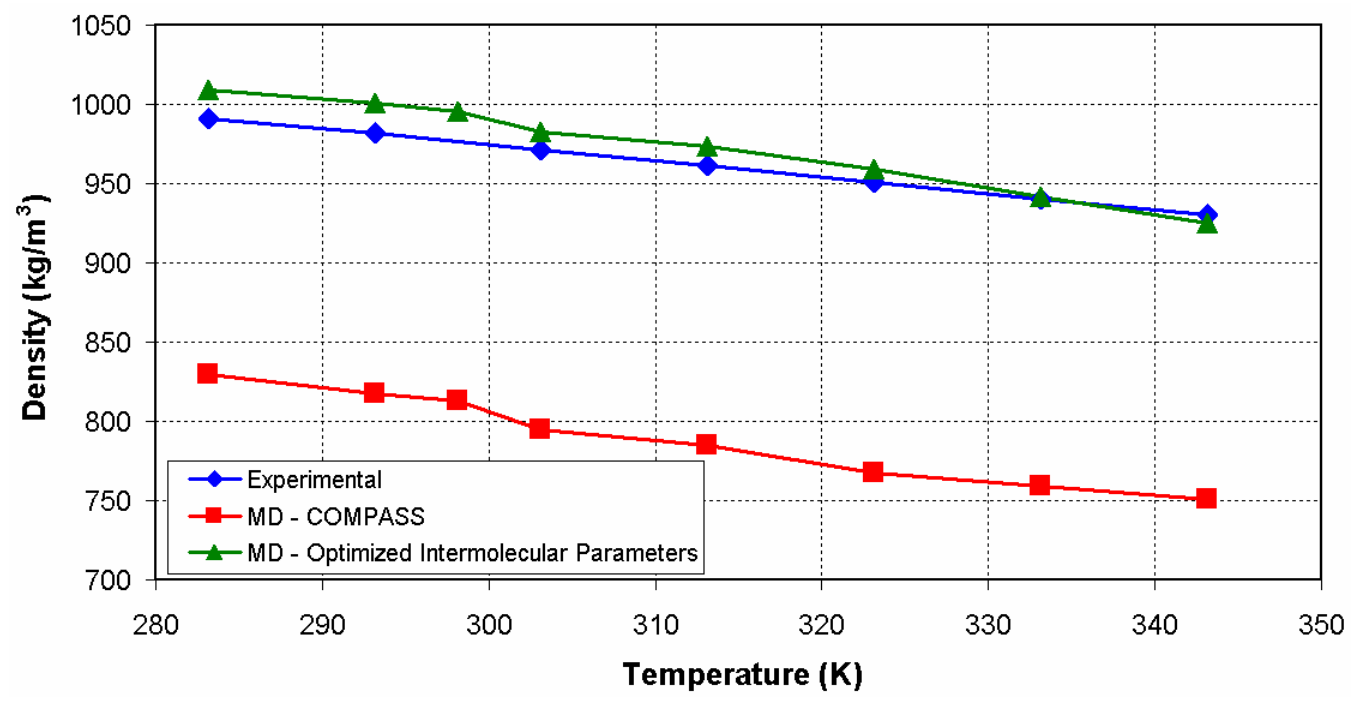

Figure 4.3: Quadricyclane Density vs. Temperature $(\mathrm{P}=1 \mathrm{~atm})$ 


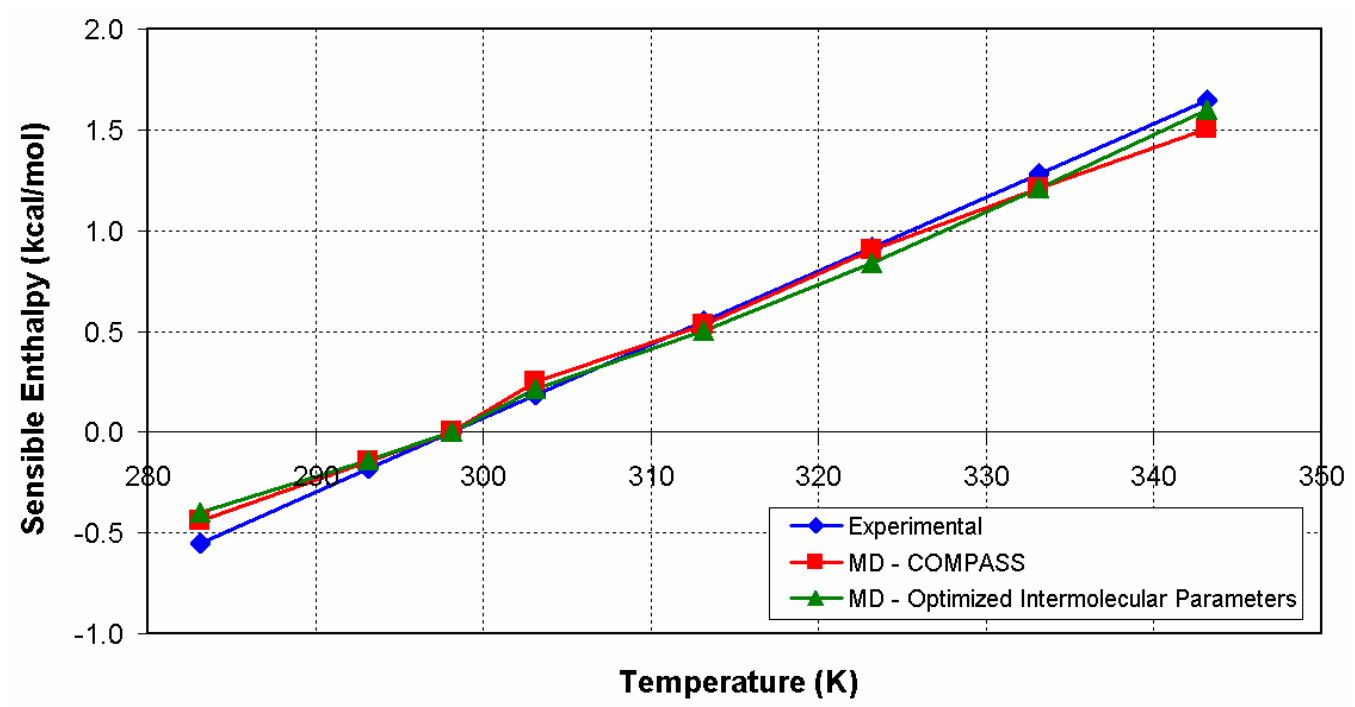

Figure 4.4: Quadricyclane Sensible Enthalpy vs. Temperature $(\mathrm{P}=1 \mathrm{~atm})$

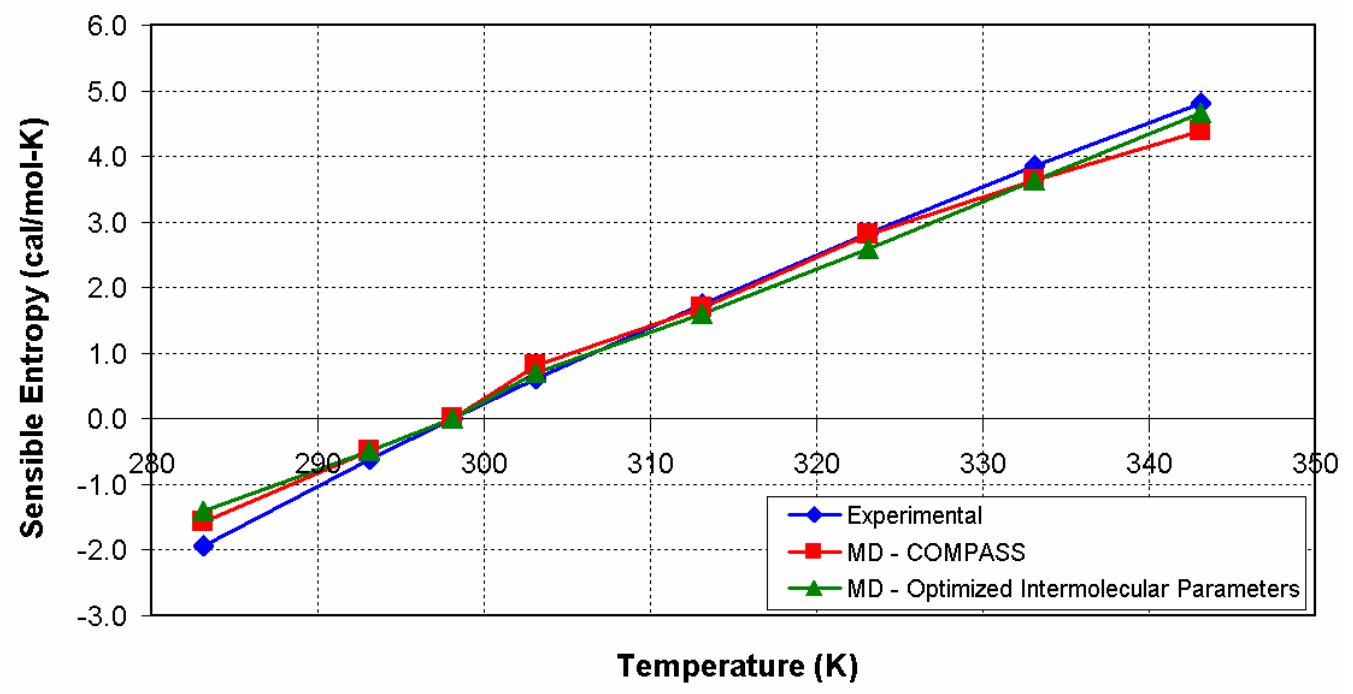

Figure 4.5: Quadricyclane Sensible Entropy vs. Temperature $(\mathrm{P}=1 \mathrm{~atm})$ 


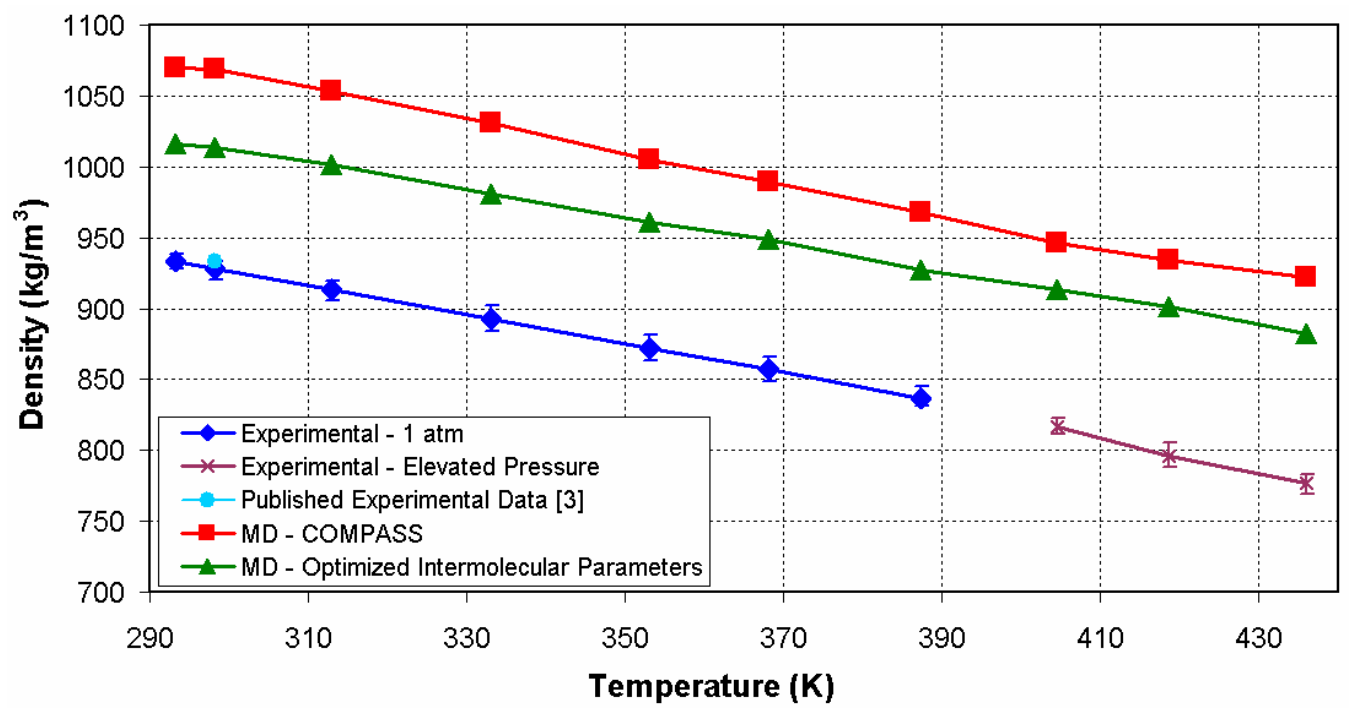

Figure 4.6: DMAZ Density vs. Temperature

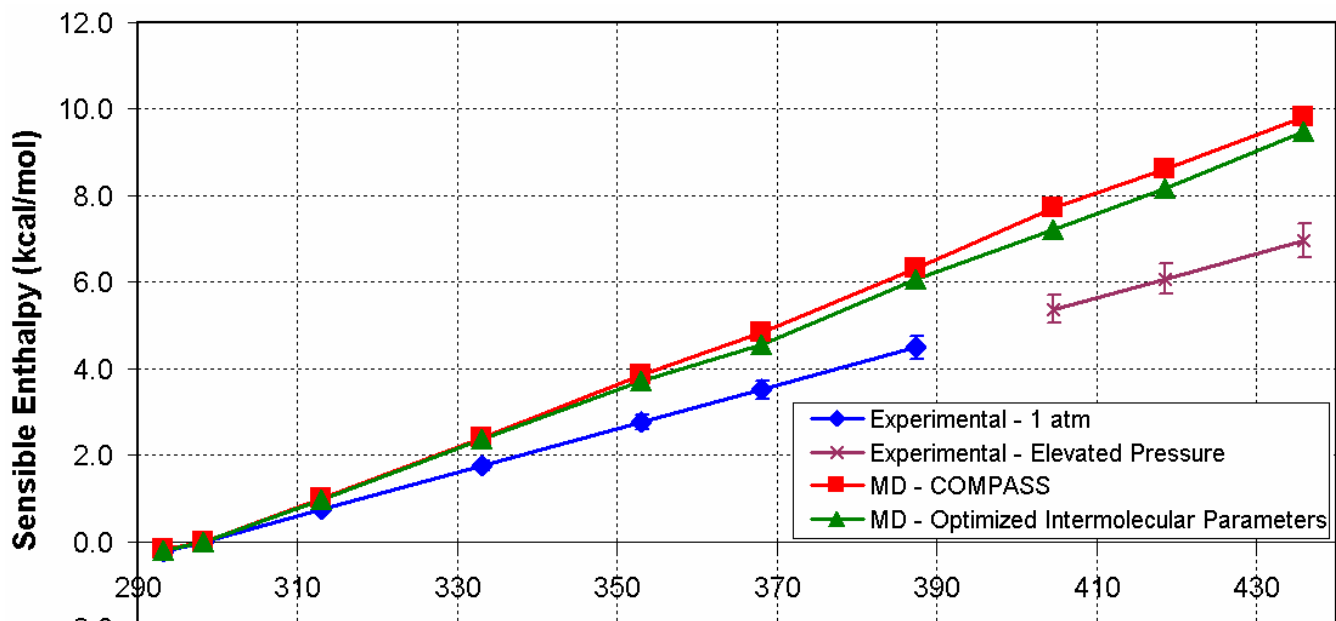

Temperature (K)

Figure 4.7: DMAZ Sensible Enthalpy vs. Temperature 


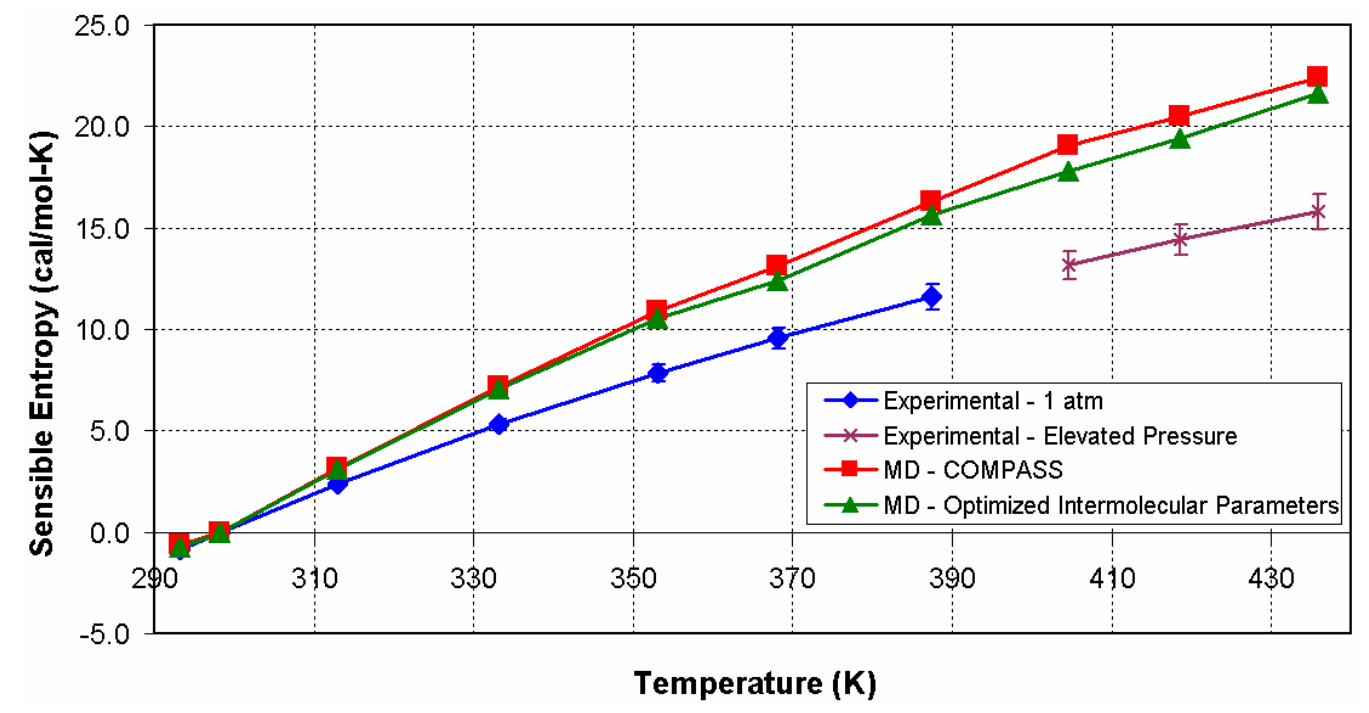

Figure 4.8: DMAZ Sensible Entropy vs. Temperature

The average RMS deviations of both the baseline COMPASS simulations and optimized intermolecular parameter simulations are provided in Table 4.29. The deviations in total enthalpy and total entropy provided in Table 4.29 are calculated by adding the sensible components shown in the above figures to the enthalpy and entropy of formation of each compound. The enthalpy and entropy of formation of each HEDM compound is provided in Table 4.30 .

Table 4.29: Average RMS Deviations of Molecular Dynamics Property Predictions

\begin{tabular}{ccc}
\hline & $\begin{array}{c}\text { COMPASS } \\
\text { Average RMS } \\
\text { Deviation }\end{array}$ & $\begin{array}{c}\text { Optimized Intermolecular } \\
\text { Parameters Average } \\
\text { RMS Deviation }\end{array}$ \\
\hline Quadricyclane & & \\
Density & $18.20 \%$ & $1.12 \%$ \\
Total Enthalpy & $0.08 \%$ & $0.08 \%$ \\
Total Entropy & $0.43 \%$ & $0.46 \%$ \\
Specific Heat (Cp) & $11.39 \%$ & $9.00 \%$ \\
\hdashline DMAZ & $15.95 \%$ & $10.95 \%$ \\
Density & $1.80 \%$ & $1.51 \%$ \\
Total Enthalpy & $6.73 \%$ & $5.67 \%$ \\
Total Entropy & $36.86 \%$ & $32.45 \%$ \\
Specific Heat (Cp) &
\end{tabular}


Table 4.30: HEDM Compound Enthalpy and Entropy of Formation

\begin{tabular}{ccc}
\hline HEDM Compound & $\Delta h_{f}^{0}(\mathrm{kcal} / \mathrm{mol})$ & $\Delta s_{f}^{\text {latm }}(\mathrm{cal} / \mathrm{mol}-\mathrm{K})$ \\
\hline Quadricyclane & $72.2[78]$ & $39.9[78]$ \\
DMAZ & $66.9[79]$ & $36.8[79]$ \\
\hline
\end{tabular}

As can be seen from Table 4.29, the average RMS deviations in density for both quadricyclane and DMAZ are reduced through the optimization of the molecular dynamics intermolecular potential function parameters. A greater improvement is shown with quadricyclane than with DMAZ. This is most likely due to three causes: (1) the lack of changes made to the charge bond parameters (the charge bond parameters for the nitrogen bonds in DMAZ are much larger than those for the carbon bonds in quadricyclane), (2) choice of model compound, and (3) quality of model compound density RSE. These causes are further discussed in section 6.2 with recommendations made for improvement.

The average RMS deviations in total enthalpy and total entropy for quadricyclane are very small for both the baseline COMPASS and optimized intermolecular simulations. The deviations in specific heat for quadricyclane are reduced through the optimization method developed in this research.

Specific heat is listed in addition to enthalpy and entropy, because it is a good indication of the accuracy in predicting the changes in these thermodynamic properties as a function of temperature and pressure. In rocket engine powerhead design, the total enthalpy is used in the combustion analysis, but only the change in entropy is used. This change in entropy is needed if one assumes that the propellant can exist as a product of the reaction as well as a reactant. If one assumes that the propellant can only exist as a reactant and not as a product, then entropy is not used in the combustion analysis.

The deviations for DMAZ are higher than those for quadricyclane. As a result, there is greater room for improvement in the predictive accuracy of the molecular 
dynamics simulations for DMAZ than for quadricyclane. Although improved accuracy is achieved for DMAZ, there is still room for further improvement and the causes outlined previously (charge bond parameters, choice of model compound, and quality of model compound RSEs) should be studied for future work. Additionally, a study of the phase change of compounds would be useful for future work, especially for applications other than conceptual rocket engine powerhead design such as avoiding pump cavitation when designing turbopumps.

\section{Data Table Simulations}

With the new intermolecular parameters validated for the two HEDM compounds, molecular dynamics simulations are performed in order to populate propellant property data tables to be used by rocket engine powerhead design codes. Table 4.31 and Table 4.32 are the results of these molecular dynamics simulations for quadricyclane and DMAZ respectively. 
Table 4.31: Quadricyclane Property Data Table Run Results

\begin{tabular}{|c|c|c|c|c|c|c|}
\hline $\begin{array}{c}\text { Temperature } \\
\text { (K) }\end{array}$ & $\begin{array}{c}\text { Pressure } \\
\text { (psi) }\end{array}$ & $\begin{array}{l}\text { Density } \\
\left(\mathrm{kg} / \mathrm{m}^{3}\right)\end{array}$ & $\begin{array}{c}\text { Sensible } \\
\text { Enthalpy } \\
\text { (kcal/mol) }\end{array}$ & $\begin{array}{c}\text { Sensible } \\
\text { Entropy } \\
\text { (cal/mol-K) }\end{array}$ & $\begin{array}{c}\text { Total } \\
\text { Enthalpy } \\
(\mathrm{kcal} / \mathrm{mol})\end{array}$ & $\begin{array}{c}\text { Total } \\
\text { Entropy } \\
(\mathrm{cal} / \mathrm{mol}-\mathrm{K})\end{array}$ \\
\hline 298.15 & 14.7 & 1001.0 & 0.0000 & 0.0000 & 72.2000 & 39.9327 \\
\hline $280^{\circ}$ & 14.7 & 1020.3 & -0.5422 & -1.9363 & 71.6578 & 37.9964 \\
\hline 280 & 500 & 1022.4 & -0.4843 & -1.9869 & 71.7157 & 37.9458 \\
\hline 280 & 1000 & 1028.7 & -0.4532 & -2.1378 & 71.7468 & 37.7949 \\
\hline 280 & 1500 & 1029.8 & -0.3863 & -2.1615 & 71.8137 & 37.7712 \\
\hline 280 & 2000 & 1031.9 & -0.3218 & -2.1921 & 71.8782 & 37.7406 \\
\hline 280 & 3000 & 1037.0 & -0.2096 & -2.3092 & 71.9904 & 37.6235 \\
\hline 280 & 5000 & 1050.0 & -0.0129 & -2.6199 & 72.1871 & 37.3128 \\
\hline 280. & 10000 & 1084.3 & 0.4267 & -3.4685 & 72.6267 & 36.4642 \\
\hline 300 & 14.7 & 996.8 & 0.0748 & 0.2493 & 72.2748 & 40.1820 \\
\hline 300 & 500 & 999.5 & 0.1302 & 0.1883 & 72.3302 & 40.1210 \\
\hline 300 & 1000 & 1007.0 & 0.1489 & 0.0012 & 72.3489 & 39.9339 \\
\hline 300 & 1500 & 1009.9 & 0.2103 & -0.0431 & 72.4103 & 39.8896 \\
\hline 300 & 2000 & 1019.3 & 0.2047 & -0.3030 & 72.4047 & 39.6297 \\
\hline 300 & 3000 & 1028.0 & 0.2889 & -0.5063 & 72.4889 & 39.4264 \\
\hline 300 & 5000 & 1035.8 & 0.5434 & -0.6239 & 72.7434 & 39.3088 \\
\hline 300 & 10000 & 1065.8 & 1.0320 & -1.3002 & 73.2320 & 38.6324 \\
\hline 350 & 14.7 & 930.0 & 1.8520 & 5.2915 & 74.0520 & 45.2242 \\
\hline 350 & 500 & 935.8 & 1.9032 & 5.2129 & 74.1032 & 45.1455 \\
\hline 350 & 1000 & 939.4 & 1.9525 & 5.1237 & 74.1525 & 45.0564 \\
\hline 350 & 1500 & 943.3 & 2.0171 & 5.0801 & 74.2171 & 45.0128 \\
\hline 350 & 2000 & 948.0 & 2.0610 & 4.9805 & 74.2610 & 44.9132 \\
\hline 350 & 3000 & 954.0 & 2.1812 & 4.8749 & 74.3812 & 44.8076 \\
\hline 350 & 5000 & 973.5 & 2.3550 & 4.5075 & 74.5550 & 44.4402 \\
\hline 350 & 10000 & 1008.2 & 2.8589 & 3.8728 & 75.0589 & 43.8054 \\
\hline 400 & 14.7 & 850.4 & 3.9660 & 9.9149 & 76.1660 & 49.8476 \\
\hline 400 & 500 & 857.1 & 4.0059 & 9.7998 & 76.2059 & 49.7325 \\
\hline 400 & 1000 & 861.3 & 4.0765 & 9.7572 & 76.2765 & 49.6899 \\
\hline 400 & 1500 & 863.3 & 4.1562 & 9.7375 & 76.3562 & 49.6702 \\
\hline 400 & 2000 & 870.3 & 4.1865 & 9.6005 & 76.3865 & 49.5332 \\
\hline 400 & 3000 & 889.1 & 4.2261 & 9.2911 & 76.4261 & 49.2238 \\
\hline 400 & 5000 & 915.9 & 4.3650 & 8.8471 & 76.5650 & 48.7798 \\
\hline 400 & 10000 & 954.8 & 4.8820 & 8.2365 & 77.0820 & 48.1691 \\
\hline
\end{tabular}


Table 4.32: DMAZ Property Data Table Run Results

\begin{tabular}{|c|c|c|c|c|c|c|}
\hline $\begin{array}{c}\text { Temperature } \\
\text { (K) }\end{array}$ & $\begin{array}{l}\text { Pressure } \\
\text { (psi) }\end{array}$ & $\begin{array}{l}\text { Density } \\
\left(\mathrm{kg} / \mathrm{m}^{3}\right)\end{array}$ & $\begin{array}{c}\text { Sensible } \\
\text { Enthalpy } \\
\text { (kcal/mol) }\end{array}$ & $\begin{array}{c}\text { Sensible } \\
\text { Entropy } \\
\text { (cal/mol-K) }\end{array}$ & $\begin{array}{c}\text { Total } \\
\text { Enthalpy } \\
(\mathrm{kcal} / \mathrm{mol})\end{array}$ & $\begin{array}{c}\text { Total } \\
\text { Entropy } \\
(\text { cal } / \text { mol-K) }\end{array}$ \\
\hline 298.15 & 14.7 & 1013.9 & 0.0000 & 0.0000 & 66.9000 & 36.8284 \\
\hline 290 & 14.7 & 1025.2 & -0.6516 & -2.2469 & 66.2484 & 34.5816 \\
\hline 290 & 500 & 1027.0 & -0.6095 & -2.4080 & 66.2905 & 34.4205 \\
\hline 290 & 1000 & 1030.3 & -0.5741 & -2.5995 & 66.3259 & 34.2290 \\
\hline 290 & 1500 & 1033.0 & -0.5336 & -2.7719 & 66.3664 & 34.0565 \\
\hline 290 & 2000 & 1034.9 & -0.4513 & -2.7997 & 66.4487 & 34.0288 \\
\hline 290 & 3000 & 1038.0 & -0.3284 & -2.9967 & 66.5716 & 33.8318 \\
\hline 290 & 5000 & 1042.8 & 0.0054 & -3.0801 & 66.9054 & 33.7483 \\
\hline 290 & 10000 & 1059.0 & 0.6495 & -3.8725 & 67.5495 & 32.9559 \\
\hline 340 & 14.7 & 974.5 & 2.7839 & 8.1880 & 69.6839 & 45.0165 \\
\hline 340 & 500 & 977.4 & 2.7869 & 7.9222 & 69.6869 & 44.7506 \\
\hline 340 & 1000 & 981.7 & 2.8173 & 7.7313 & 69.7173 & 44.5597 \\
\hline 340 & 1500 & 986.5 & 2.7792 & 7.3416 & 69.6792 & 44.1700 \\
\hline 340 & 2000 & 989.4 & 2.8024 & 7.1329 & 69.7024 & 43.9614 \\
\hline 340 & 3000 & 992.1 & 2.9140 & 6.9068 & 69.8140 & 43.7352 \\
\hline 340 & 5000 & 996.8 & 3.3561 & 7.1058 & 70.2561 & 43.9343 \\
\hline 340 & 10000 & 1014.5 & 3.9543 & 6.1886 & 70.8543 & 43.0170 \\
\hline 390 & 14.7 & 929.0 & 6.1075 & 15.6602 & 73.0075 & 52.4886 \\
\hline 390 & 500 & 935.2 & 6.1553 & 15.5328 & 73.0553 & 52.3612 \\
\hline 390 & 1000 & 936.4 & 6.1541 & 15.2726 & 73.0541 & 52.1011 \\
\hline 390 & 1500 & 940.0 & 6.2517 & 15.2684 & 73.1517 & 52.0968 \\
\hline 390 & 2000 & 944.7 & 6.2457 & 15.0017 & 73.1457 & 51.8302 \\
\hline 390 & 3000 & 948.8 & 6.3814 & 14.8459 & 73.2814 & 51.6743 \\
\hline 390 & 5000 & 952.2 & 6.7489 & 14.7814 & 73.6489 & 51.6098 \\
\hline 390 & 10000 & 975.1 & 7.3588 & 13.9325 & 74.2588 & 50.7610 \\
\hline 435 & 14.7 & 886.9 & 9.3077 & 21.3970 & 76.2077 & 58.2255 \\
\hline 435 & 500 & 888.6 & 9.4200 & 21.4192 & 76.3200 & 58.2476 \\
\hline 435 & 1000 & 891.5 & 9.4813 & 21.3186 & 76.3813 & 58.1470 \\
\hline 435 & 1500 & 894.4 & 9.5386 & 21.2102 & 76.4386 & 58.0386 \\
\hline 435 & 2000 & 899.4 & 9.5539 & 21.0091 & 76.4539 & 57.8376 \\
\hline 435 & 3000 & 907.1 & 9.6767 & 20.8232 & 76.5767 & 57.6516 \\
\hline 435 & 5000 & 916.0 & 9.9696 & 20.5667 & 76.8696 & 57.3952 \\
\hline 435 & 10000 & 940.2 & 10.5278 & 19.6124 & 77.4278 & 56.4408 \\
\hline
\end{tabular}

\subsubsection{ADDITIVITY METHODS}

Using the density predictions for the HEDM compounds and the definition of the molecule (atoms, bonds, groups), additivity methods are used to predict the kinematic viscosity and thermal conductivity of each HEDM compound as a function of temperature and pressure. 


\section{Comparison with Experimental Data}

Additivity method calculations are first performed at the temperature and pressure combinations at which experimental data exists. Similarly to the molecular dynamics predictions of density, enthalpy, and entropy, these additivity method calculations are performed in order to verify the accuracy of these methods with respect to experimental results.

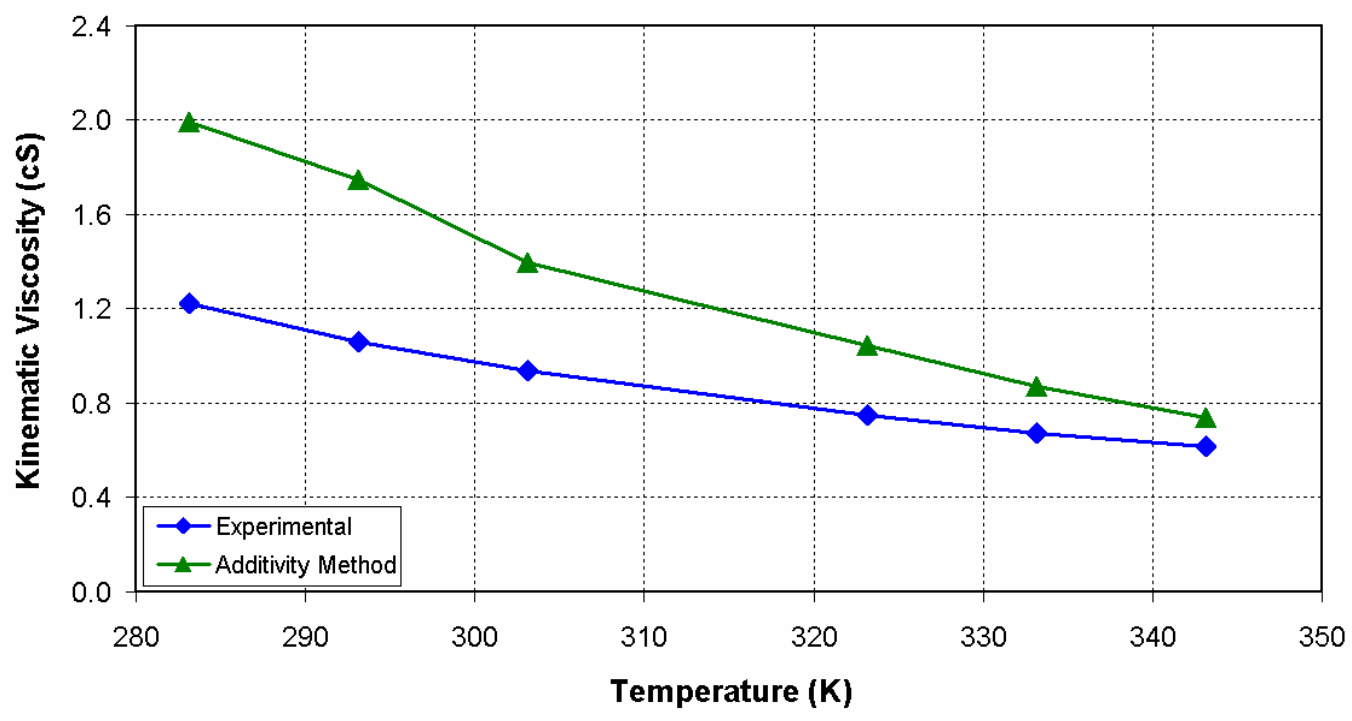

Figure 4.9: Quadricyclane Kinematic Viscosity vs. Temperature ( $\mathrm{P}=1 \mathrm{~atm})$

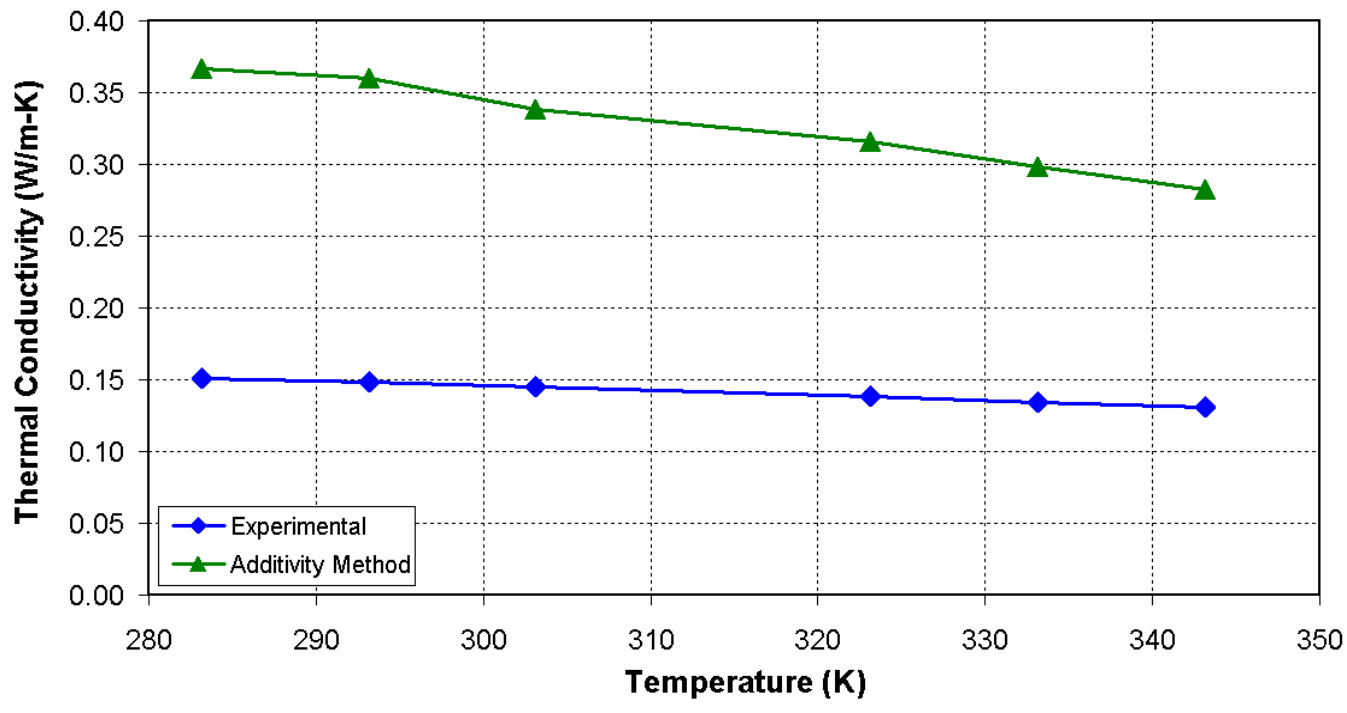

Figure 4.10: Quadricyclane Thermal Conductivity vs. Temperature ( $\mathrm{P}=1 \mathrm{~atm})$ 


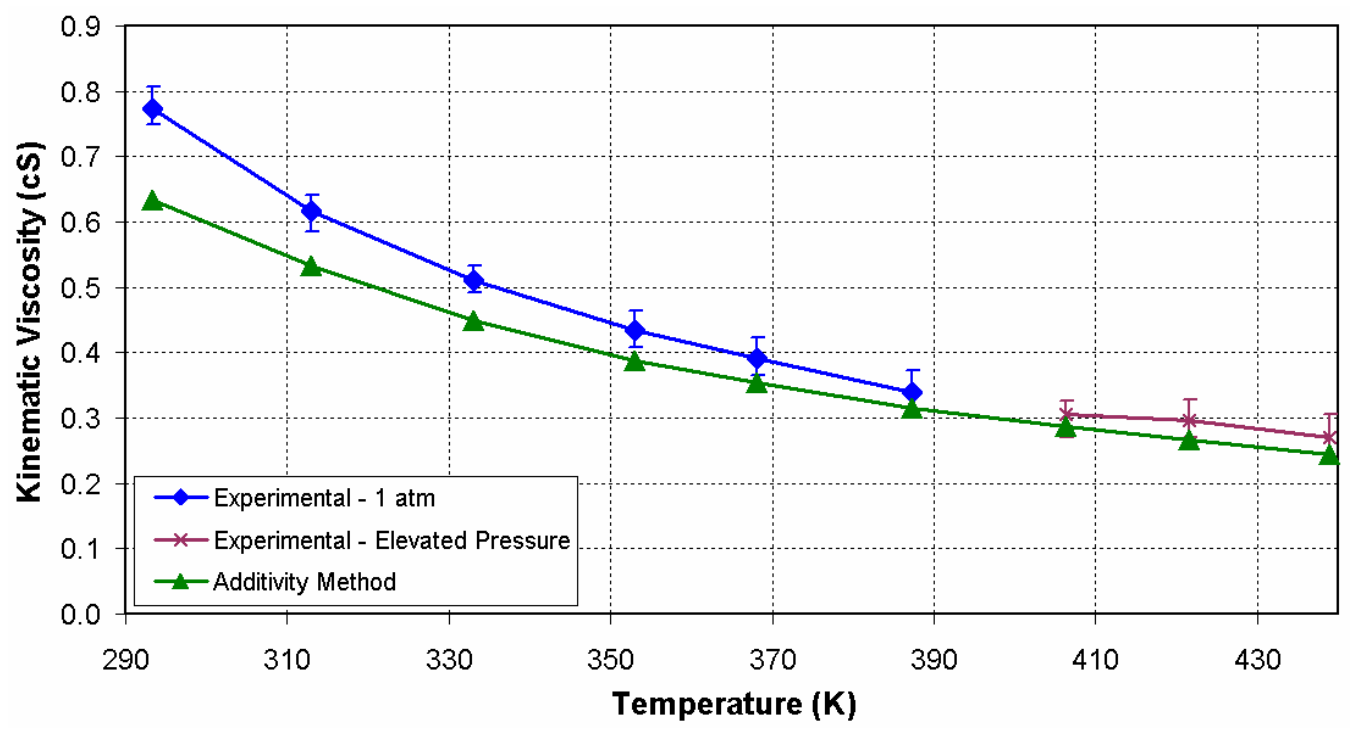

Figure 4.11: DMAZ Kinematic Viscosity vs. Temperature

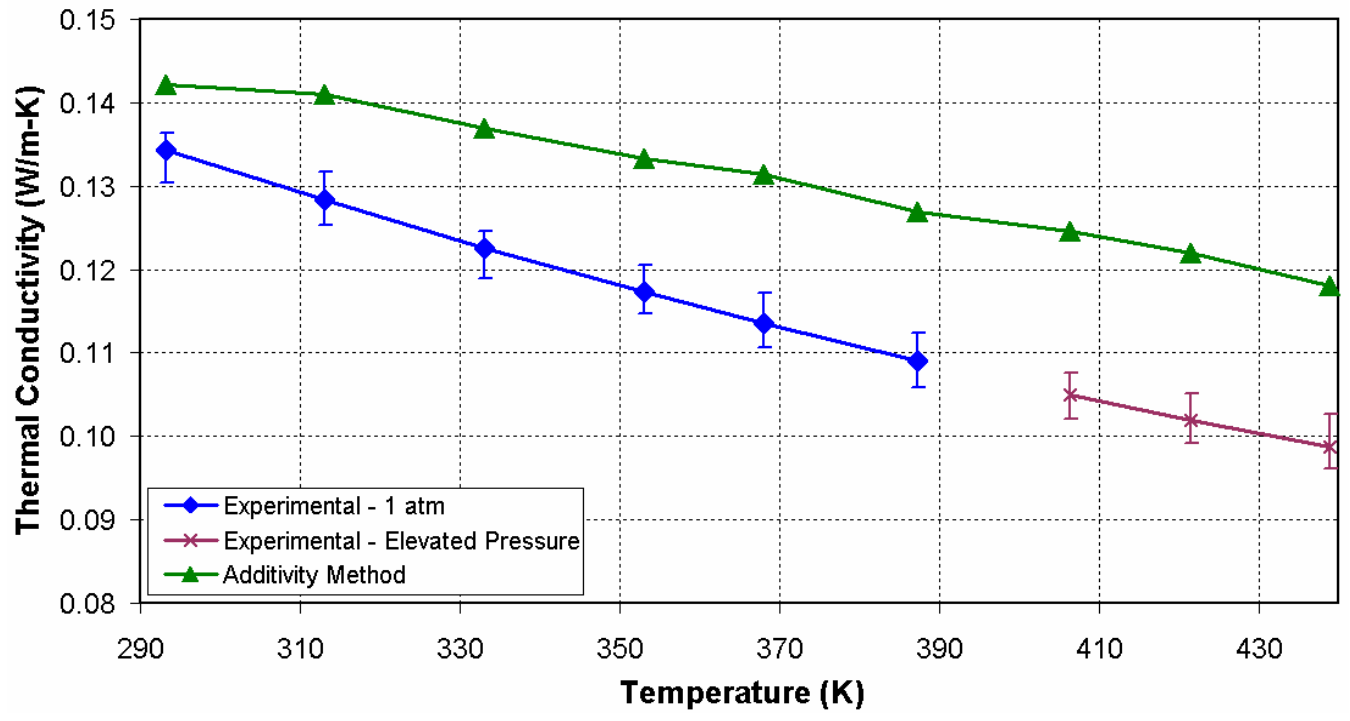

Figure 4.12: DMAZ Thermal Conductivity vs. Temperature

Similarly to the DMAZ results for density, enthalpy, and entropy, experimental measurements and corresponding additivity method calculations are performed at a range of pressures. The experimental measurements of DMAZ at the three highest 
temperatures were performed at elevated pressures in order to suppress boiling. Minimum and maximum bars are provided for the DMAZ experimental measurements.

The average RMS deviations of the additivity method calculations are provided in Table 4.33. The average RMS deviations for these properties are much larger than for density, enthalpy, and entropy. These higher RMS deviations for kinematic viscosity and thermal conductivity are fine for the application of rocket engine powerhead design because the sensitivity of $I_{\mathrm{sp}}$ to changes/errors in these parameters is very small (as was shown in section 1.3). Even an error of over $100 \%$, like that seen in the thermal conductivity of quadricyclane, has a minimal impact on $\mathrm{I}_{\mathrm{sp}}$, as shown in Figure 1.6. If greater accuracy is needed in the prediction of these properties, other calculation techniques could potentially be employed. Potential improvements in calculating these properties are discussed in Section 6.2.

Table 4.33: Additivity Method Average RMS Deviation

\begin{tabular}{cc}
\hline & $\begin{array}{c}\text { Additivity Method } \\
\text { Average RMS Deviation }\end{array}$ \\
\hline Quadricyclane & \\
Kinematic Viscosity & $45.64 \%$ \\
Thermal Conductivity & $129.73 \%$ \\
\hline DMAZ & $10.81 \%$ \\
Kinematic Viscosity & $14.59 \%$ \\
Thermal Conductivity & \\
\hline
\end{tabular}

\section{Data Table Calculations}

With the additivity method calculations validated for quadricyclane and DMAZ, data tables are populated at the same temperature and pressure combinations used in the data table population from molecular dynamics simulations. With the addition of kinematic viscosity and thermal conductivity additivity method predictions to the density, enthalpy, and entropy predictions from molecular dynamics simulations, all the necessary 
thermophysical properties will be predicted for use in rocket engine powerhead design codes.

Table 4.34: Quadricyclane Additivity Method Data Table Results

\begin{tabular}{|c|c|c|c|}
\hline $\begin{array}{c}\text { Temperature } \\
\text { (K) }\end{array}$ & $\begin{array}{c}\text { Pressure } \\
\text { (psi) }\end{array}$ & $\begin{array}{c}\text { Kinematic } \\
\text { Viscosity } \\
(c S)\end{array}$ & $\begin{array}{c}\text { Thermal } \\
\text { Conductivity } \\
(\mathrm{W} / \mathrm{m}-\mathrm{K})\end{array}$ \\
\hline 298.15 & 14.7 & 1.7262 & 0.3632 \\
\hline 280 & 14.7 & 2.3055 & 0.3830 \\
\hline 280 & 500 & 2.3659 & 0.3866 \\
\hline 280 & 1000 & 2.5580 & 0.3975 \\
\hline 280 & 1500 & 2.5932 & 0.3994 \\
\hline 280 & 2000 & 2.6626 & 0.4031 \\
\hline 280 & 3000 & 2.8401 & 0.4123 \\
\hline 280 & 5000 & 3.3705 & 0.4371 \\
\hline 280 & 10000 & 5.4483 & 0.5109 \\
\hline 300 & 14.7 & 1.6377 & 0.3578 \\
\hline 300 & 500 & 1.6885 & 0.3621 \\
\hline 300 & 1000 & 1.8393 & 0.3741 \\
\hline 300 & 1500 & 1.9041 & 0.3789 \\
\hline 300 & 2000 & 2.1304 & 0.3949 \\
\hline 300 & 3000 & 2.3712 & 0.4103 \\
\hline 300 & 5000 & 2.6171 & 0.4247 \\
\hline 300 & 10000 & 3.9178 & 0.4861 \\
\hline 350 & 14.7 & 0.7609 & 0.2914 \\
\hline 350 & 500 & 0.7981 & 0.2984 \\
\hline 350 & 1000 & 0.8238 & 0.3031 \\
\hline 350 & 1500 & 0.8516 & 0.3080 \\
\hline 350 & 2000 & 0.8887 & 0.3143 \\
\hline 350 & 3000 & 0.9381 & 0.3222 \\
\hline 350 & 5000 & 1.1344 & 0.3501 \\
\hline 350 & 10000 & 1.6586 & 0.4067 \\
\hline 400 & 14.7 & 0.4083 & 0.2253 \\
\hline 400 & 500 & 0.4241 & 0.2314 \\
\hline 400 & 1000 & 0.4346 & 0.2354 \\
\hline 400 & 1500 & 0.4398 & 0.2373 \\
\hline 400 & 2000 & 0.4587 & 0.2442 \\
\hline 400 & 3000 & 0.5174 & 0.2635 \\
\hline 400 & 5000 & 0.6282 & 0.2943 \\
\hline 400 & 10000 & 0.8755 & 0.3462 \\
\hline
\end{tabular}


Table 4.35: DMAZ Additivity Method Data Table Results

\begin{tabular}{|c|c|c|c|}
\hline $\begin{array}{c}\text { Temperature } \\
\text { (K) }\end{array}$ & $\begin{array}{l}\text { Pressure } \\
\text { (psi) }\end{array}$ & $\begin{array}{c}\text { Kinematic } \\
\text { Viscosity } \\
(c S)\end{array}$ & $\begin{array}{c}\text { Thermal } \\
\text { Conductivity } \\
(W / m-K)\end{array}$ \\
\hline 298.15 & 14.7 & 0.60794 & 0.14252 \\
\hline 290 & 14.7 & 0.66705 & 0.14522 \\
\hline 290 & 500 & 0.67043 & 0.14598 \\
\hline 290 & 1000 & 0.67669 & 0.14740 \\
\hline 290 & 1500 & 0.68189 & 0.14858 \\
\hline 290 & 2000 & 0.68559 & 0.14941 \\
\hline 290 & 3000 & 0.69171 & 0.15077 \\
\hline 290 & 5000 & 0.70136 & 0.15292 \\
\hline 290 & 10000 & 0.73579 & 0.16037 \\
\hline 340 & 14.7 & 0.42713 & 0.13603 \\
\hline 340 & 500 & 0.43032 & 0.13719 \\
\hline 340 & 1000 & 0.43512 & 0.13892 \\
\hline 340 & 1500 & 0.44058 & 0.14088 \\
\hline 340 & 2000 & 0.44394 & 0.14208 \\
\hline 340 & 3000 & 0.44710 & 0.14320 \\
\hline 340 & 5000 & 0.45269 & 0.14518 \\
\hline 340 & 10000 & 0.47486 & 0.15288 \\
\hline 390 & 14.7 & 0.31306 & 0.12810 \\
\hline 390 & 500 & 0.31774 & 0.13043 \\
\hline 390 & 1000 & 0.31866 & 0.13088 \\
\hline 390 & 1500 & 0.32144 & 0.13226 \\
\hline 390 & 2000 & 0.32513 & 0.13408 \\
\hline 390 & 3000 & 0.32841 & 0.13569 \\
\hline 390 & 5000 & 0.33117 & 0.13704 \\
\hline 390 & 10000 & 0.35078 & 0.14647 \\
\hline 435 & 14.7 & 0.25110 & 0.12017 \\
\hline 435 & 500 & 0.25205 & 0.12076 \\
\hline 435 & 1000 & 0.25369 & 0.12178 \\
\hline 435 & 1500 & 0.25535 & 0.12281 \\
\hline 435 & 2000 & 0.25826 & 0.12461 \\
\hline 435 & 3000 & 0.26283 & 0.12742 \\
\hline 435 & 5000 & 0.26830 & 0.13076 \\
\hline 435 & 10000 & 0.28413 & 0.14026 \\
\hline
\end{tabular}




\section{CHAPTER 5}

\section{CONCEPTUAL VEHICLE DESIGN CASE STUDY}

A conceptual vehicle design case study is performed using the resulting thermophysical property values calculated from Chapter 4 . The vehicle chosen for this case study is the Lunar Surface Access Module (LSAM) used in the NASA Exploration Systems Architecture Study (ESAS) [80]. This vehicle is chosen for study because the propellant choice for the ascent stage of the LSAM is still undetermined. The current baseline propellant combination for the ascent stage is NTO-MMH. A propellant trade study is performed to compare the NTO-MMH baseline propellant combination with a currently proposed alternative of LOX-Methane and two HEDM propellant combinations: LOX-DMAZ and LOX-Quadricyclane. A rendering of this vehicle, taken from the ESAS Final Report [80], is shown in Figure 5.1.

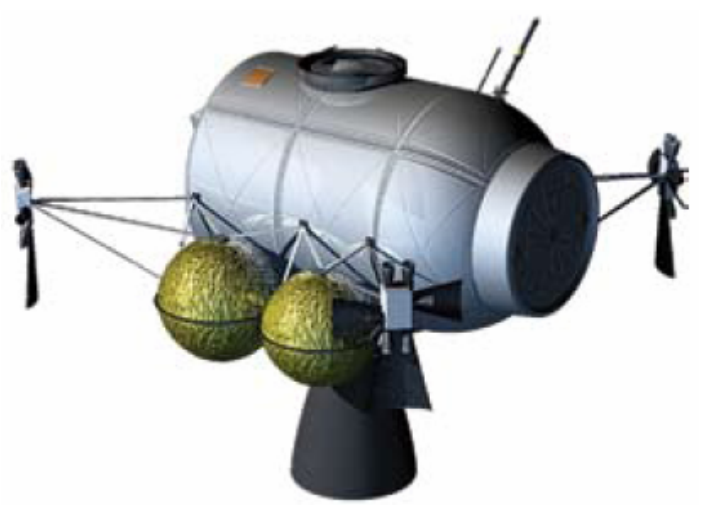

(a)

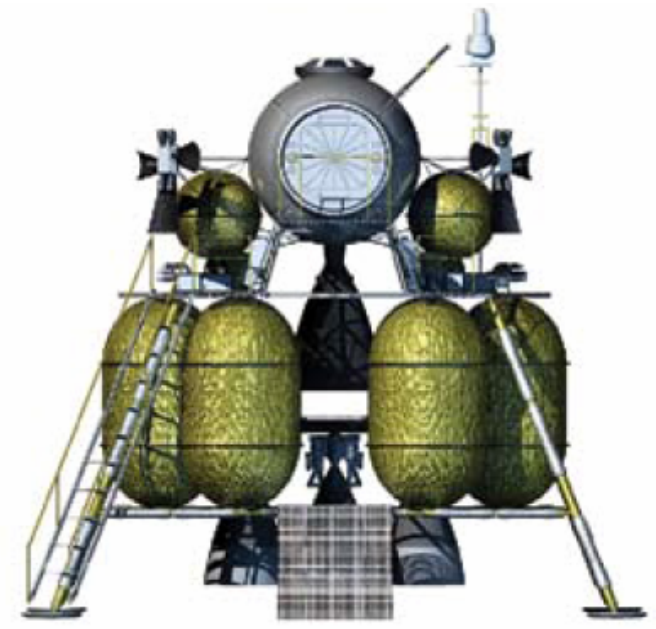

(b)

Figure 5.1: ESAS Baseline LSAM [80]

(a) Ascent stage

(b) Ascent + Descent Stages 
The ESAS report details the mission requirements for a crew plus cargo lunar mission [80]. An overview of the mission requirements is provided in Table 5.1. An important item in this mission parameter list is the requirement that the LSAM be able to orbit the Earth for up to 120 days before beginning the mission. This requirement is in place in order to allow several opportunities for the crew launch vehicle to launch and rendezvous with the previously launched orbiting cargo (including the LSAM).

Table 5.1: ESAS Mission Parameters

\begin{tabular}{lc}
\hline \multicolumn{1}{c}{ Parameter } & Value \\
\hline Number of Crew & 4 \\
Payload to Lunar Surface & $2395 \mathrm{~kg}$ \\
Mission Time & 7 days \\
On-orbit Time & 120 days \\
Dry Weight Margin & $20 \%$ \\
\hline
\end{tabular}

This requirement makes the LSAM propellant choices that much more important, because cryogenic propellant boiloff can be significant over such an extended period of time. Cryogenic propellants will boil off during long duration storage because of the environmental heating of the propellant tanks. As a result, extra propellant is needed to accommodate these losses. This extra propellant also requires larger propellant tanks [81].

Two typical methods of minimizing propellant boiloff are insulation and cryocoolers. Typical cryogenic propellant tank insulation is a multilayer insulation (MLI) consisting of alternating layers of aluminized Mylar and Nomex [82, p.438, 83, p.273]. This method is advantageous as it is a passive system requiring no power to operate. However, this type of system typically only reduces propellant boiloff; it does not completely eliminate it. Crycoolers are some sort of refrigeration system, often based on a Stirling or Brayton cycle, used to reach cryogenic temperatures [82, p.442]. This method of propellant cooling is advantageous because it has the ability to be a zero- 
boiloff system when used in combination with insulation. The main disadvantage of this system is that it requires power to operate and can be fairly heavy. In this case study, both methods of propellant boiloff minimization are examined for the different propellant combinations.

\subsection{DisCIPLINES}

In a general conceptual space vehicle design, a variety of discipline analyses are needed. These disciplines include configuration \& CAD, aerodynamics, propulsion, trajectory, weights \& sizing, safety \& reliability, operations, and cost \& economics. For this case study, only the propulsion, trajectory, and weights \& sizing disciplines were analyzed. The economics loop (safety \& reliability, operations, and cost \& economics) was ignored as the goal of this case study is to show the performance benefits of a new propellant combination. Configuration \& CAD is ignored as the configuration is already set and only small perturbations to the baseline are made. Aerodynamics is ignored as there are no aerodynamic forces on the lunar lander.

Figure 5.2 is a design structure matrix (DSM) of the disciplines considered. A DSM is useful in graphically displaying both the disciplines being analyzed and the information that must be passed between disciplines. The black dots in Figure 5.2 represent data that must be transferred from one discipline to another. For instance, the propulsion discipline transfers the specific impulse $\left(\mathrm{I}_{\mathrm{sp}}\right)$ determined through a powerhead design analysis to the trajectory discipline. The trajectory discipline uses the $I_{\mathrm{sp}}$ to determine the propellant mass flow rate needed for a particular thrust level. The dots in the upper-right represent feed-forward links of data while the dots in the lower-left represent feedback links of data, which require iteration to converge to a solution.

The propulsion analysis is performed using REDTOP-2 [64], a powerhead design and analysis code. This code uses a propellant data table text file for all thermophysical propellant properties used by the code. In order to incorporate the propellant data for 
quadricyclane and DMAZ, new data table text files were created for use in REDTOP-2. The trajectory analysis is performed using the Program to Optimize Simulated Trajectories - 3D (POST-3D) [84]. POST-3D is an industry-standard three degree-offreedom trajectory optimization code. The weights \& sizing analysis is performed using industry standard mass estimating relationships (MER) [85].

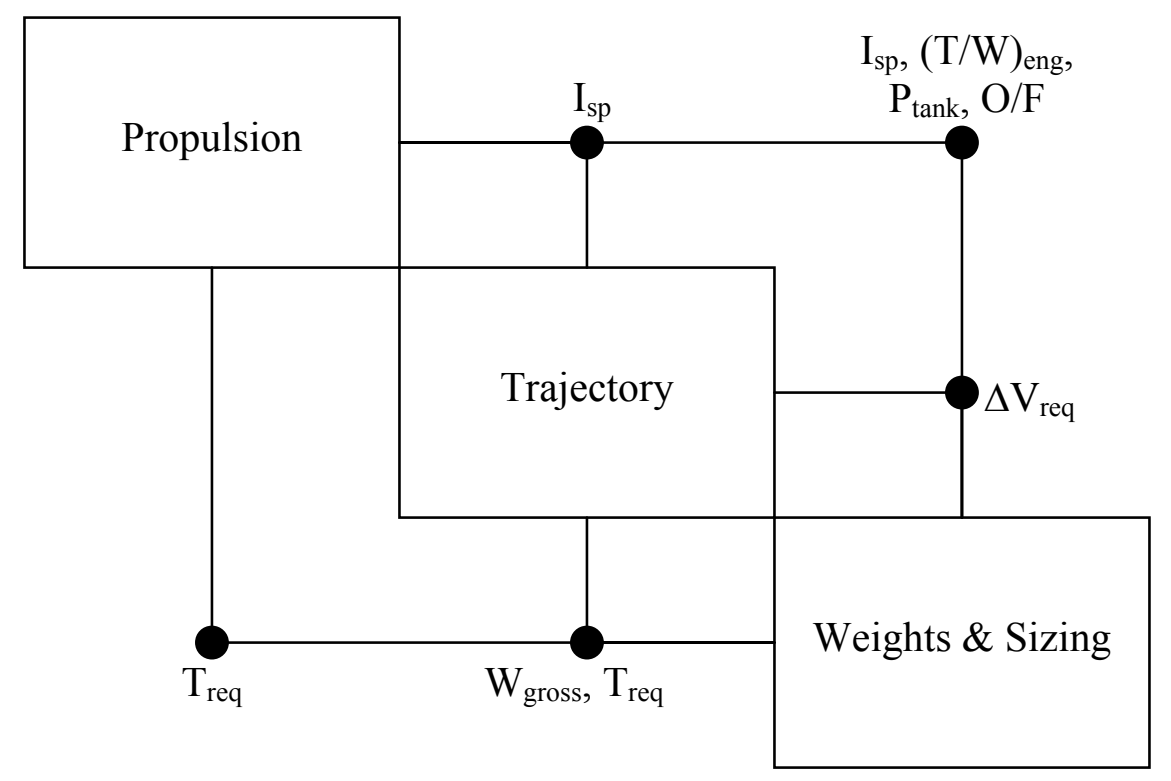

Figure 5.2: Design Structure Matrix of Conceptual Space Vehicle Design

\subsection{ReSUlts}

The results of the four different propellant combinations (1 - NTO-MMH, 2 LOX-Methane, 3 - LOX-DMAZ, and 4 - LOX-Quadricyclane) indicate that, from a performance point of view, the three alternatives to the current NTO-MMH design all hold potential for overall LSAM gross mass savings.

Table 5.2 provides a comparison of the design and performance characteristics of the four different ascent stage engine alternatives. The same cycle type (Expander), expansion ratio (84), and chamber pressure (610 psi) are used for each engine [86]. These choices are based upon the RL-10A-4 liquid rocket engine design. This engine is 
chosen as a high performance alternative to pressure-fed engines in order to produce a lighter weight LSAM vehicle allowing for a smaller cargo launch vehicle to be used to launch the LSAM and its associated Earth-departure stage into low-Earth orbit (LEO).

Table 5.2: Ascent Stage Engine Comparison

\begin{tabular}{lcccc}
\hline & Baseline & $\begin{array}{c}\text { Currently } \\
\text { Proposed } \\
\text { Alternative }\end{array}$ & $\begin{array}{c}\text { HEDM } \\
\text { Alternative \#1 }\end{array}$ & $\begin{array}{c}\text { HEDM } \\
\text { Alternative \#2 }\end{array}$ \\
\hline Oxidizer & NTO & LOX & LOX & LOX \\
Fuel & MMH & Methane & Quadricyclane & DMAZ \\
Mixture Ratio - O/F & 1.6 & 3.1 & 2.0 & 1.4 \\
Cycle Type & Expander & Expander & Expander & Expander \\
Expansion Ratio $(\varepsilon)$ & 84 & 84 & 84 & 84 \\
$P_{\text {Chamber }}(\mathrm{psi})$ & 610 & 610 & 610 & 610 \\
$\rho_{\text {Oxidizer }}\left(\mathrm{kg} / \mathrm{m}^{3}\right)$ & 1450 & 1141 & 1141 & 1141 \\
$\rho_{\text {Fuel }}\left(\mathrm{kg} / \mathrm{m}^{3}\right)$ & 880 & 420 & 1025 & 1030 \\
$\rho_{\text {Bulk }}\left(\mathrm{kg} / \mathrm{m}^{3}\right)$ & 1231 & 965 & 1102 & 1095 \\
$\mathrm{~T}_{\text {Oxidizer }}(\mathrm{R})$ & 536.67 & 163 & 163 & 163 \\
$\mathrm{~T}_{\text {Fuel }}(\mathrm{R})$ & 536.67 & 250 & 536.67 & 536.67 \\
Engine T/W & 48.3 & 44.9 & 49.6 & 49.7 \\
$\mathrm{I}_{\text {sp }}(\mathrm{sec})$ & 330.35 & 365.97 & 357.43 & 354.77 \\
\hline
\end{tabular}

As can be seen from the last row in Table 5.2, the three alternative propellant combinations all have a higher $\mathrm{I}_{\mathrm{sp}}$ than the NTO-MMH baseline. The mixture ratios chosen for the three alternatives are the peak $I_{\mathrm{sp}}$ mixture ratios for this engine configuration.

Table 5.3 and Table 5.4 are the mass results for the baseline propellant combination and the three possible propellant alternatives for the two propellant boiloff minimization methods. In both cases (MLI and MLI + cryocooler), the alternative propellant combinations have a lower overall LSAM gross mass than the NTO-MMH baseline. In the case where only MLI is used, the two HEDM alternatives provide more mass savings than the LOX-Methane alternative. When MLI + cryocoolers are used (a more aggressive propellant boiloff minimization method), then the LOX-Methane 
alternative becomes more attractive. This is because the LOX-Methane alternative has both cryogenic fuel and oxidizer where as the two HEDM alternatives have only cryogenic oxidizers. As a result, the LOX-Methane alternative is more sensitive to the type of boiloff minimization method used.

Table 5.3: LSAM Mass Results Comparison - MLI

\begin{tabular}{lcccc}
\hline & NTO- & LOX- & LOX- & LOX- \\
& $M M H$ & Methane & Quadricyclane & DMAZ \\
\hline Ascent Dry Mass (kg) & 4,660 & 4,810 & 4,690 & 4,690 \\
Ascent Takeoff Mass (kg) & 9,580 & 9,460 & 9,330 & 9,350 \\
Descent Dry Mass (kg) & 5,690 & 5,670 & 5,630 & 5,630 \\
Descent Gross Mass (kg) & 27,920 & 27,700 & 27,430 & 27,470 \\
Ascent Stage Takeoff Mass & $0.00 \%$ & $1.27 \%$ & $2.67 \%$ & $2.41 \%$ \\
Savings Compared to Baseline & & & & \\
\hline
\end{tabular}

Table 5.4: LSAM Mass Results Comparison - MLI + Cryocooler

\begin{tabular}{lccc}
\hline & $\begin{array}{c}\text { LOX- } \\
\text { Methane }\end{array}$ & $\begin{array}{c}\text { LOX- } \\
\text { Quadricyclane }\end{array}$ & $\begin{array}{c}\text { LOX- } \\
\text { DMAZ }\end{array}$ \\
\hline Ascent Dry Mass (kg) & 4,730 & 4,650 & 4,650 \\
Ascent Takeoff Mass (kg) & 9,160 & 9,140 & 9,180 \\
Descent Dry Mass (kg) & 5,590 & 5,580 & 5,590 \\
Descent Gross Mass (kg) & 27,120 & 27,070 & 27,140 \\
Ascent Stage Takeoff Mass & $4.38 \%$ & $4.58 \%$ & $4.17 \%$ \\
Savings Compared to Baseline & & & \\
\hline
\end{tabular}

The LOX-Quadricyclane alternative has the lowest LSAM gross weight for both boiloff minimization methods. This is due to a combination of high $\mathrm{I}_{\mathrm{sp}}$ and high bulk density. Bulk density is defined using the following equation:

$$
\rho_{\text {Bulk }}=\rho_{\text {Oxidizer }} * \frac{O / F}{O / F+1}+\rho_{\text {Fuel }} * \frac{1}{O / F+1}
$$


In the above equation, $\rho_{\text {Oxidizer }}$ is the oxidizer density, $\rho_{\text {Fuel }}$ is the fuel density, and $\mathrm{O} / \mathrm{F}$ is the oxidizer-to-fuel mixture ratio (by mass).

The LOX-Quadricyclane $\mathrm{I}_{\mathrm{sp}}$ and bulk density are both higher than that of LOXDMAZ. The LOX-Quadricyclane $\mathrm{I}_{\mathrm{sp}}$ is lower than that of LOX-Methane, but the significantly higher bulk density more than makes up for the slightly lower $\mathrm{I}_{\mathrm{sp}}$. These results indicate that the use of HEDM propellants may be an attractive option for the LSAM ascent stage engine. Further analysis including cost, operations, reliability, and safety should be done to further flush out the relative advantages and disadvantages of HEDM propellant combinations over NTO-MMH and LOX-Methane.

\subsection{COMPARISON WITH EXISTING METHODS}

A comparison of the results from the previous section can be made with those found using the current method of conceptual vehicle design using new propellants with very little thermophysical data. To use the current method, the enthalpy of formation and storage density for the new propellant must be known. This data is typically found from published results.

With this information, a one-dimensional chemical equilibrium analysis code can be used to get a prediction of the $\mathrm{I}_{\mathrm{sp}}$. Analysis codes such as CEA [87] and REDTOP [88] are examples of this type of analysis code. One-dimensional chemical equilibrium analysis codes typically only calculate the ideal $\mathrm{I}_{\mathrm{sp}}$ with no losses. REDTOP is the rare exception in which an efficiency multiplier can be applied to the ideal $\mathrm{I}_{\text {sp }}$ to attempt to account for these losses. This multiplier is based upon regressed existing engine performance data and not on any engine component analysis. As a result of being regressed from existing engine performance data, these efficiency multipliers, which depend upon the propellant type among other factors, are not generally applicable to new propellant combinations. 
Another drawback of this type of analysis code is the inability to calculate the engine weight directly by estimating individual component weights. The engine thrustto-weight is typically either an input or calculated from a technique developed by Way et al. [89]. This technique calculated engine thrust-to-weight using the following equations:

$$
\begin{gathered}
\frac{T_{v a c}}{W_{\text {Eng }}}=\frac{k \times T_{v a c}}{\text { Power }} \\
\text { Power }=\dot{m}_{\text {prop }} \times\left(h_{c}-h_{e}\right) \\
k=520 \frac{B T U}{l b f-s}
\end{gathered}
$$

In the above equations, $\left(T_{\text {vac }} / W_{E n g}\right)$ is the vacuum thrust-to-weight; $T_{\text {vac }}$ is the vacuum thrust in units of lbf; $\dot{m}_{\text {prop }}$ is the propellant mass flow rate through the nozzle in units of $\mathrm{lbm} / \mathrm{s} ; h_{c}$ and $h_{e}$ are the combustor and exhaust plane enthalpies respectively in units of $\mathrm{BTU} / \mathrm{lbm}$; $\mathrm{k}$ is a multiplication factor based upon regressed existing engine data used to predict engine thrust-to-weight in units of BTU/lbf-s.

The LSAM was designed using this engine analysis technique, using REDTOP as the chemical equilibrium analysis code in place of a rocket engine powerhead design code. A comparison of engine results is provided for both the REDTOP and REDTOP-2 results in Table 5.5. As can be seen from Table 5.5, the estimations for engine thrust-toweight are significantly different between the two engine analysis codes. It is assumed that the engine thrust-to-weights from REDTOP-2 are more accurate as the analysis is based on an estimation of engine weight at the component level (turbopump, combustor, valves, propellant feed lines, etc.). The $\mathrm{I}_{\mathrm{sp}}$ results from REDTOP are fairly close (between 1-5 second difference) to those from REDTOP-2 for the LOX-Methane, LOX- 
Quadricyclane, and LOX-DMAZ engines. However, the $I_{\text {sp }}$ results are significantly different for the NTO-MMH engine (15.5 second difference). This is likely due to the fact that there is no expander cycle NTO-MMH data in the REDTOP database used to calculate engine efficiencies. Assuming the results from REDTOP-2 are more accurate as the engine losses are estimated at the component level instead of the REDTOP method of simply applying an engine efficiency multiplier, then the REDTOP results indicate an inaccurately large performance difference between the baseline NTO-MMH engine and the other three engines.

Table 5.5: Comparison of Engine Analysis Code Results

\begin{tabular}{|c|c|c|c|c|}
\hline & Baseline & $\begin{array}{c}\text { Currently } \\
\text { Proposed } \\
\text { Alternative }\end{array}$ & $\begin{array}{c}\text { HEDM } \\
\text { Alternative \#1 }\end{array}$ & $\begin{array}{c}\text { HEDM } \\
\text { Alternative \#2 }\end{array}$ \\
\hline \multicolumn{5}{|c|}{ REDTOP-2 Powerhead Design Code } \\
\hline Engine $\mathrm{T} / \mathrm{W}$ & 48.3 & 44.9 & 49.6 & 49.7 \\
\hline $\mathrm{I}_{\mathrm{sp}}(\mathrm{sec})$ & 330.35 & 365.97 & 357.43 & 354.77 \\
\hline \multicolumn{5}{|c|}{ REDTOP Chemical Equilibrium Code } \\
\hline Engine $\mathrm{T} / \mathrm{W}$ & 76.6 & 67.9 & 68.8 & 69.5 \\
\hline $\mathrm{I}_{\mathrm{sp}}(\mathrm{sec})$ & 314.81 & 361.38 & 355.51 & 353.74 \\
\hline
\end{tabular}

Table 5.6 and Table 5.7 provide a comparison of the vehicle weight predictions using each engine analysis method. As expected from the previous analysis of engine $\mathrm{I}_{\mathrm{sp}}$, the baseline NTO-MMH combination has a larger weight difference compared with the alternative propellant combinations for the REDTOP analysis case. This is interpreted as an artificial penalization of the NTO-MMH combination due to a lower fidelity engine analysis model. If one were making funding choices based upon a conceptual vehicle design analysis, one may be more inclined to fund research into alternative propellant combinations for the LSAM ascent stage engine based upon the REDTOP results than upon the REDTOP-2 results. 
Table 5.6: Comparison of Vehicle Results - MLI

\begin{tabular}{|c|c|c|c|c|}
\hline & $\begin{array}{l}\text { NTO- } \\
M M H\end{array}$ & $\begin{array}{l}\text { LOX- } \\
\text { Methane }\end{array}$ & $\begin{array}{c}L O X- \\
\text { Quadricyclane }\end{array}$ & $\begin{array}{l}L O X- \\
D M A Z\end{array}$ \\
\hline \multicolumn{5}{|c|}{ REDTOP-2 Powerhead Design Code } \\
\hline Ascent Takeoff Mass (kg) & 9,580 & 9,460 & 9,330 & 9,350 \\
\hline Descent Gross Mass (kg) & 27,920 & 27,700 & 27,430 & 27,470 \\
\hline $\begin{array}{l}\text { Ascent Stage Takeoff Mass } \\
\text { Savings Compared to } \\
\text { Baseline }\end{array}$ & $0.00 \%$ & $1.27 \%$ & $2.67 \%$ & $2.41 \%$ \\
\hline \multicolumn{5}{|c|}{ REDTOP Chemical Equilibrium Code } \\
\hline Ascent Takeoff Mass (kg) & 9,800 & 9,440 & 9,290 & 9,300 \\
\hline Descent Gross Mass (kg) & 28,350 & 27,670 & 27,350 & 27,380 \\
\hline $\begin{array}{l}\text { Ascent Stage Takeoff Mass } \\
\text { Savings Compared to } \\
\text { Baseline }\end{array}$ & $0.00 \%$ & $3.72 \%$ & $5.24 \%$ & $5.09 \%$ \\
\hline
\end{tabular}

Table 5.7: Comparison of Vehicle Results - MLI + Cryocooler

\begin{tabular}{|c|c|c|c|}
\hline & $\begin{array}{c}\text { LOX- } \\
\text { Methane }\end{array}$ & $\begin{array}{c}\text { LOX- } \\
\text { Quadricyclane }\end{array}$ & $\begin{array}{c}L O X^{-} \\
D M A Z\end{array}$ \\
\hline \multicolumn{4}{|c|}{ REDTOP-2 Powerhead Design Code } \\
\hline Ascent Takeoff Mass (kg) & 9,160 & 9,140 & 9,180 \\
\hline Descent Gross Mass (kg) & 27,120 & 27,070 & 27,140 \\
\hline $\begin{array}{l}\text { Ascent Stage Takeoff Mass } \\
\text { Savings Compared to } \\
\text { Baseline }\end{array}$ & $4.38 \%$ & $4.58 \%$ & $4.17 \%$ \\
\hline \multicolumn{4}{|c|}{ REDTOP Chemical Equilibrium Code } \\
\hline Ascent Takeoff Mass (kg) & 9,130 & 9,100 & 9,130 \\
\hline Descent Gross Mass (kg) & 27,060 & 26,980 & 27,040 \\
\hline $\begin{array}{l}\text { Ascent Stage Takeoff Mass } \\
\text { Savings Compared to } \\
\text { Baseline }\end{array}$ & $6.81 \%$ & $7.14 \%$ & $6.81 \%$ \\
\hline
\end{tabular}




\section{CHAPTER 6}

\section{CONCLUSIONS AND RECOMMENDATIONS}

The goal of this research project was to develop a thermophysical property calculation method which can be used to calculate the properties necessary for the conceptual design of rocket engine powerheads. This goal was achieved through the use of a variety of property prediction methods including quantum mechanical energy calculations, molecular dynamics simulations, and group additivity calculation methods.

\subsection{ConClusions}

This goal had associated with it three specific objectives listed in section 1.5. These objectives are repeated below along with a summary of how and to what degree each one was achieved.

\section{Objective 1: The process of predicting the thermophysical properties of potential new liquid rocket propellants should primarily be analytical / numerical. It should require little or no new experimental work.}

This objective was included because this property calculation method must be able to be incorporated into a conceptual design environment in which a limited amount of time and resources are available. In conceptual design, where a turn around time on a design is typically measured in days, experimental measurements are very difficult to perform. As was the case with this research work, even simply obtaining a sample of HEDM propellant can be quite time consuming. As a result, the property prediction process requires no new experimental work. Model compounds with existing published 
experimental data are used to determine the most appropriate values for the intermolecular potential parameters used in molecular dynamics simulations. In this thesis research work, experimental data for the two HEDM compounds was used to validate the process and quantify its accuracy. However, in general application of this property prediction process, no new experimental data is needed.

Objective 2: Predict the density of HEDM molecules to within $10 \%$ of the experimentally measured value. Predict the total enthalpy and total entropy to within 5\% of the experimentally measured value. Predict the specific heat at constant pressure to within $10 \%$ of the experimentally measured value. Do this by improving the predictive capability of the COMPASS force field model.

A combination of quantum mechanical energy calculations and molecular dynamics simulations was used to predict the density, enthalpy, and entropy of HEDM molecules. Quantum mechanics was used to determine the minimum energy molecular configuration and the normal mode vibrational frequencies of both a model compound and its corresponding HEDM compound. The normal mode vibrational frequencies were used to calculate the ideal gas component of the specific heat. Molecular dynamics was used to predict the density and residual contribution (non-ideal gas or liquid contribution) to specific heat.

Model compounds were used in this process in order to have some experimental data against which to compare molecular dynamics simulation results, because in general very little experimental data exists for HEDM compounds. A set of rules was developed for choosing a particular model compound. These compounds have a similar molecular structure to their corresponding HEDM compounds and some experimental density and specific heat data. 
Molecular dynamics was used initially to determine the appropriate values for the parameters used in the intermolecular terms of the COMPASS model. These values were determined by comparing the results of molecular dynamics simulations of the model compounds with experimental data.

Response surface equations were created describing the relationship between these intermolecular parameter values and both density and specific heat of the model compounds. The intermolecular parameter values were then optimized using a gradientbased optimizer in order to minimize the difference between the molecular dynamics predicted values and experimental values.

Once validated, these updated intermolecular parameter values were then used in molecular dynamics simulations of each model compound's corresponding HEDM compound. Predictions of density, enthalpy, and entropy were made over a range of temperatures and pressures experienced in liquid rocket engines. The results were compared against experimentally measured data for both quadricyclane and DMAZ. The data for quadricyclane was found in the literature while the data for DMAZ was obtained through experimental measurements performed as part of this research work.

Table 6.1 provides a summary of the average deviations in the prediction of density, total enthalpy, and total entropy for the two HEDM compounds analyzed. Results are provided for the baseline COMPASS model and for the optimized intermolecular parameter model. As can be seen, the average deviations from the optimized intermolecular parameter simulations are lower for quadricyclane than for DMAZ. The average deviations for quadricyclane for all four thermophysical properties are lower than the objective. However, for DMAZ, only the objective for total enthalpy is met. The average deviation for the density and total entropy of DMAZ is slightly above the objective in both cases. The average deviation for specific heat is significantly above the objective. 
Table 6.1: Molecular Dynamics Average Deviations

\begin{tabular}{|c|c|c|c|}
\hline & $\begin{array}{c}\text { COMPASS } \\
\text { Average } R M S \\
\text { Deviation } \\
\end{array}$ & $\begin{array}{c}\text { Optimized Intermolecular } \\
\text { Parameters Average RMS } \\
\text { Deviation }{ }^{\dagger}\end{array}$ & Objective \\
\hline \multicolumn{4}{|l|}{ Quadricyclane } \\
\hline Density & $18.20 \%$ & $1.12 \%$ & $<10 \%$ \\
\hline Total Enthalpy & $0.08 \%$ & $0.08 \%$ & $<5 \%$ \\
\hline Total Entropy & $0.43 \%$ & $0.46 \%$ & $<5 \%$ \\
\hline Specific Heat $(\mathrm{Cp})$ & $11.39 \%$ & $9.00 \%$ & $<10 \%$ \\
\hline \multicolumn{4}{|l|}{ DMAZ } \\
\hline Density & $15.95 \%$ & $10.95 \%$ & $<10 \%$ \\
\hline Total Enthalpy & $1.80 \%$ & $1.51 \%$ & $<5 \%$ \\
\hline Total Entropy & $6.73 \%$ & $5.67 \%$ & $<5 \%$ \\
\hline Specific Heat (Cp) & $36.86 \%$ & $32.45 \%$ & $<10 \%$ \\
\hline $\begin{array}{l}\text { Calculations of tota } \\
\text { of formation. Calcu } \\
\text { mechanically predic } \\
\text { experimentally mea } \\
\text { the liquid entropy o }\end{array}$ & $\begin{array}{l}\text { alpy use ex } \\
\text { ns for total } \\
\text { alues for } g \\
\text { values for } \\
\text { hation [78, }\end{array}$ & $\begin{array}{l}\text { nentally measured values } \\
\text { py use a combination of } \\
\text { s entropy of formation a } \\
\text { nthalpy of vaporization }\end{array}$ & enthalpy \\
\hline
\end{tabular}

These results indicate that the process developed in this thesis research worked very well for quadricyclane but not as well for DMAZ. While some improvements are made in the prediction of density, total enthalpy, and total entropy of DMAZ, there is still significant room for further improvement.

Some possible reasons for the lack of accuracy in the prediction of the thermophysical properties of DMAZ are the choice of model compound and the fit of the density and specific heat RSEs. These points are further discussed in the following section along with suggestions for improvement.

Another interesting result is that the baseline COMPASS model performed fairly well at predicting the total enthalpy and total entropy of both quadricyclane and DMAZ. In the case of quadricyclane, the baseline COMPASS model satisfied the objectives without any need for parameter optimization. This result is due to the fact that the values for the formation enthalpy and formation entropy (obtained either from literature or from 
quantum mechanical energy analysis), which play a significant role in the calculation of total enthalpy and total entropy respectively, are the same for both the COMPASSpredicted and optimized intermolecular parameter-predicted enthalpy and entropy.

\section{Objective 3: Predict the kinematic viscosity and thermal conductivity of HEDM molecules so that the predicted values fall within a multiplication range of $40 \%$ to $250 \%$ of the experimental values.}

This objective is much less stringent than the previous objective due to the fact that $I_{\mathrm{sp}}$ is much less sensitive to changes in kinematic viscosity and thermal conductivity than it is to changes in density, enthalpy, and entropy. Despite this fact, a certain level of accuracy is still needed in the prediction of these properties; we do not want to be off by orders of magnitude, because errors of that magnitude can have an impact on the prediction of $I_{\mathrm{sp}}$.

Group additivity method calculations are used to predict the values of kinematic viscosity and thermal conductivity over a range of temperatures and pressures. Additivity methods make use of the observation that a substance's physical properties depend on that substance's particular molecular structure. As a result, experimental data for a variety of compounds can be regressed in order to quantify the contributions to certain thermodynamic and physical properties from individual bonds, atoms, and atom groups. Table 6.2 provides a summary of the average deviations in the prediction of kinematic viscosity and thermal conductivity of the two HEDM compounds analyzed. This table also provides the multiplier required to go from the experimental value to the predicted value. This multiplier must be within the range of $40 \%-250 \%$ to satisfy Objective 3. 
Table 6.2: Additivity Method Average Deviations

\begin{tabular}{cccc}
\hline & $\begin{array}{c}\text { Additivity Method } \\
\text { Average RMS Deviation }\end{array}$ & Multiplier & $\begin{array}{c}\text { Multiplier } \\
\text { Objective }\end{array}$ \\
\hline Quadricyclane & & & \\
Kinematic Viscosity & $45.64 \%$ & $145.64 \%$ & $40 \%-250 \%$ \\
Thermal Conductivity & $129.73 \%$ & $229.73 \%$ & $40 \%-250 \%$ \\
\hline DMAZ & $10.81 \%$ & & \\
Kinematic Viscosity & $14.59 \%$ & $114.59 \%$ & $40 \%-250 \%$ \\
Thermal Conductivity & & & \\
\hline
\end{tabular}

As can be seen from Table 6.2, additivity methods do an adequate job of predicting these physical properties for HEDM compounds and meet the objectives in all cases. For liquid rocket engine applications, these deviations are sufficiently small. However, for other applications in which the prediction of kinematic viscosity and thermal conductivity require more accuracy, different prediction techniques would need to be employed. A discussion of alternative prediction techniques is provided in section 6.2.

\subsection{RECOMMENDATIONS FOR FUTURE WORK}

Opportunities exist for future work to apply, modify, and improve the property calculation method. The following is a list of seven potential opportunities for future work (listed in order of importance).

\section{Analyze other potential new rocket propellants.}

While quadricyclane and DMAZ were examined as part of this thesis work, many other potential new rocket propellants exist. Propellants with azide chains similar to DMAZ, such as CPAZ $\left(\mathrm{C}_{5} \mathrm{H}_{10} \mathrm{~N}_{4}\right)$ and MMAZ $\left(\mathrm{C}_{3} \mathrm{H}_{8} \mathrm{~N}_{4}\right)$ [3], are two potential new propellants that could provide improved performance over DMAZ. The Army Research 
Laboratory has developed techniques for synthesizing these propellants and has interest in determining their performance characteristics when burned with inhibited red fuming nitric acid (IRFNA) [3]. As a result, these are the first two new propellants that should be examined using the methods developed in this thesis work. Ball and cylinder renderings of CPAZ and MMAZ are provided in Figure 6.1.

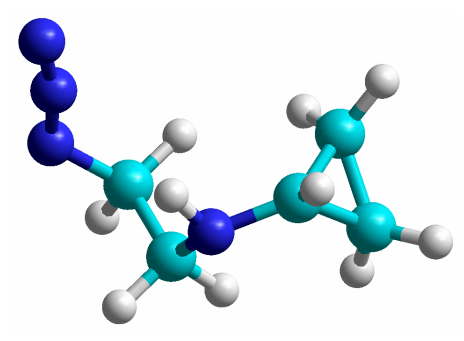

(a)

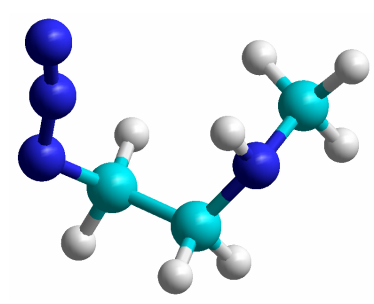

(b)

Figure 6.1: Ball and Cylinder Rendering of (a) CPAZ and (b) MMAZ

Cubane $\left(\mathrm{C}_{8} \mathrm{H}_{8}\right)$ is a molecule that has been widely studied theoretically as a potential new propellant (Figure 6.2) [30]. Quantum calculations of cubane indicate that it has an extremely high energy content and may yield a potentially high $\mathrm{I}_{\mathrm{sp}}$ when used as a rocket fuel. Difficulties synthesizing this compound [30] make it a more long-term potential rocket fuel than fuels such as DMAZ, CPAZ, and MMAZ that have received much more experimental and computational attention $[3,4]$.

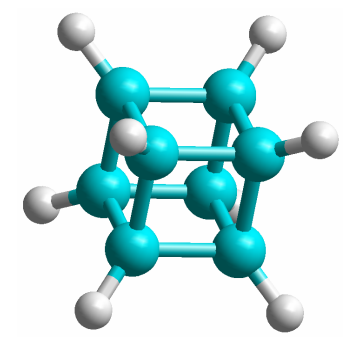

Figure 6.2: Ball and Cylinder Rendering of Cubane 
A class of compounds called silanes, are another potential set of new rocket propellants [90]. These compounds are quasi-stable silicon hydrides with high heats of formation. Preliminary equilibrium combustion calculations indicate a potentially high $I_{s p}$ when burned with hydrogen peroxide [90]. An example silane, tetrapropynyl silane $\left(\mathrm{SiC}_{12} \mathrm{H}_{12}\right)$ is provided in Figure 6.3.

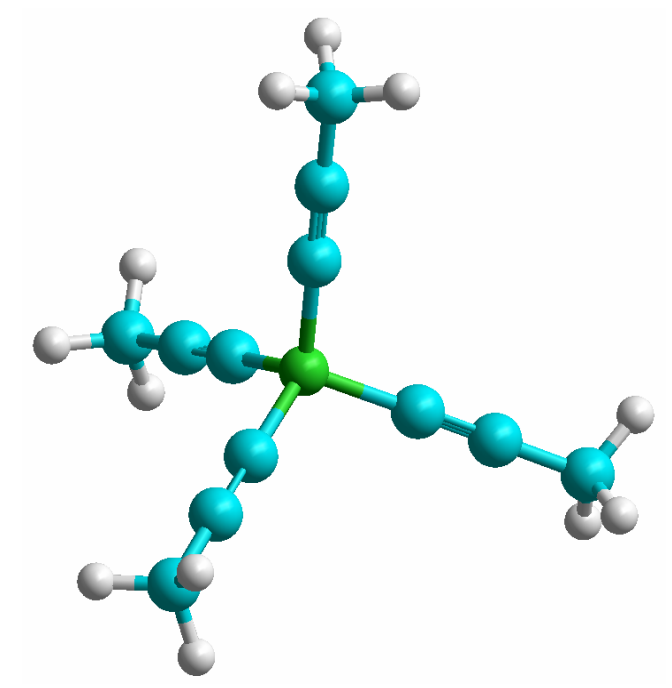

Figure 6.3: Ball and Cylinder Rendering of Tetrapropynyl Silane

\section{Do not use model compounds in the process of adjusting the intermolecular} potential parameters. Use quantum mechanics to determine these values.

As computing power increases, quantum mechanical calculations become a more and more attractive alternative for determining the proper values for the intermolecular potential parameters. Currently, quantum mechanical calculations are typically performed on a single molecule. While a great deal of information can be gained from these calculations (minimum energy molecular configurations, vibrational normal mode frequencies, intramolecular potential energies), one cannot determine the values for the intermolecular parameters as there is only one molecule. A quantum mechanical 
simulation with several molecules would be able to model the energy surface of the entire system. An analysis of this energy surface could potentially yield predictions for the intermolecular potential parameters used in molecular dynamics simulations.

Using quantum mechanics would eliminate the need to use model compounds to determine the appropriate values for these intermolecular potential parameters. This would remove many of the problems which occur when using model compounds (choosing the correct model compound, performing time-consuming DOE runs, and creating accurate RSEs of the DOE results).

\section{Incorporate the adjustment of charge bond parameters into the intermolecular parameter optimization.}

In this research work, only the Lennard-Jones $\sigma$ and $\varepsilon$ potential parameters were adjusted in order to improve the predictive accuracy of molecular dynamics simulations. An additional set of parameters describing the electrostatic forces between atoms, the charge bond increments, could also be adjusted in an attempt to improve the ability of molecular dynamics simulations to accurately match experimental results. The inaccuracy in the results for DMAZ may be due to poorly parameterized charge bond increments for the nitrogen-containing bonds. As can be seen in Table 4.9 and Table 4.10, the charge bond increments for these nitrogen-containing bonds are much larger than those for the carbon-containing bonds. These larger charge bond increments result in a larger electrostatic force associated with the nitrogen atoms and a larger influence on the simulated density and energy.

\section{Provide additional guidelines for selecting model compounds.}


While the previous future work suggestion is a long-term solution to improving the calculation method, this suggestion and the next are more appropriate short-term solutions. The predictions of density, enthalpy, and entropy for DMAZ could potentially improve with a better choice of model compound. While ethyl azide, the model compound of DMAZ used in this research work, satisfies the three rules listed in Chapter 3 for selecting a model compound, other potential model compounds exist (i.e. methyl azide) and may prove to be more suitable in this property prediction process.

Perhaps a requirement that the size of the model compound (either determined by molecular weight, number of atoms, or some length scale) be comparable to the corresponding HEDM compound would help to better define the most appropriate model compound. The reasoning behind using size as a factor is that in the liquid phase, the volume taken up by the structure of each molecule (space between atoms in a given molecule) is on the same scale as the volume between the molecules. The intermolecular potential mainly affects the spacing between molecules, not the spacing between atoms within a molecule. The space between atoms within a molecule is determined more by the intramolecular potential.

\section{Wrap an optimizer around the molecular dynamics simulation of the model compounds instead of performing DOE runs, creating density and specific heat RSE's, and optimizing the intermolecular potential parameters from the RSE's.}

Instead of creating RSE's, the optimizer would adjust the intermolecular potential parameter values directly in the molecular dynamics runs. This improvement would eliminate problems with creating an accurate RSE of a potentially complex design space. This new technique for determining the optimal settings of the intermolecular potential parameters would become more appealing as computational power increases, because the 
new technique would likely require more molecular dynamics simulations than the current method.

\section{Look into the problems molecular dynamics has with computing intramolecular potential energies.}

In this research work, quantum mechanical energy calculations were used to compute this energy. This energy was then added to the intermolecular potential energy results from molecular dynamics simulations in order to calculate the enthalpy and entropy of the compound of interest at a given temperature and pressure. If molecular dynamics could be used to accurately calculate the intramolecular potential energy, then one would be able to get both the intramolecular and intermolecular potential energies from the same simulation method. As discussed in section 3.3.6, this would allow one to capture the influence of the intermolecular potential on the intramolecular potential and vice versa. This influence, while shown to be small for benzene in section 3.3.6, could potentially be more significant for other molecules and at other temperatures and pressures.

\section{Improve the accuracy in calculating kinematic viscosity and thermal conductivity by using molecular dynamics to predict these values.}

Improved accuracy may be needed for applications other than rocket engine powerhead design. Using molecular dynamics to calculate these parameters will become more practical as computing power increases due to the fact that simulations used to predict kinematic viscosity and thermal conductivity generally require more iterations (and more computational time) than those used to predict density and specific heat [54, p.204]. 


\section{REFERENCES}

1. Wucherer, E.J. and A. Wilson. "Chemical, Physical and Hazards Properties of Quadricyclane." U.S. Air Force Research Laboratory, Edwards Air Force Base, California. PL-TR-97-3053, March 1998.

2. Tishkoff, J.M. and M.R. Berman. "Air Force Basic Research in Propellants and Combustion." U.S. Air Force Office of Scientific Research, Arlington, Virginia. AIAA Paper 2002-0901, January 2002.

3. McQuaid, M.J. “Computational Characterization of 2-Azidocycloalkanamines Notional Variations on the Hypergol 2-Azido-N,N-Dimethylethanamine (DMAZ)." U.S. Army Research Lab. JANNAF 30th Propellant Development and Chracterization Subcommittee Meeting, pp. 45-57, March 2002.

4. McQuaid, M.J. "Structure of Secondary 2-Azidoethanamines: A Hypergolic Fuel vs. a Nonhypergolic Fuel." U.S. Army Research Laboratory, Aberdeen Proving Ground, MD. ARL-TR-3176, April 2004.

5. Bai, S.D., Dumbacher, P., and J.W. Cole. "Development of Advanced Hydrocarbon Fuels at Marshall Space Flight Center." NASA/TP - 2002-211729, May 2002.

6. Palaszewski, B. "Solid Hydrogen Experiments for Atomic Propellants: Particle Formation, Imaging, Observations, and Analyses." NASA Glenn Research Center, Cleveland, Ohio. AIAA Paper 2003-4688, July 2003.

7. Palaszewski, B., Jurns, J., Breisacher, K., and K. Kearns. "Metallized Gelled Propellants Combustion Experiments in a Pulse Detonation Engine." NASA Glenn Research Center, Cleveland, Ohio. AIAA 2004-4191, July 2004.

8. Sun, H., "COMPASS: An ab Initio Force-Field Optimized for Condensed-Phase Applications - Overview with Details on Alkane and Benzene Compounds," Journal of Physical Chemistry B, 102: 7338-7364, 1998.

9. McQuaid, M.J., H. Sun, and D. Rigby, "Development and Validation of COMPASS Force Field Parameters for Molecules with Aliphatic Azide Chains," Journal of Computational Chemistry, 25: 61-71, 2004.

10. Rapaport, D.C., The Art of Molecular Dynamics Simulation, Second Edition, Cambridge University Press. Cambridge, United Kingdom, 2004.

11. Allinger, N.L., "Conformational Analysis, 130. MM2, A Hydrocarbon Force Field Utilizing $\mathrm{V}_{1}$ and $\mathrm{V}_{2}$ Torsional Terms," Journal of the American Chemical Society, Vol. 99, No. 25: 8127-8134, 1977. 
12. Blaney, J.M., P.K. Weiner, A. Dearing, P.A. Kollman, E.C. Jorgensen, S.J. Oatley, J.M. Burridge, and C.F. Blake, "Molecular Mechanics Simulation of Protein-Ligand Interactions: Binding of Thyroid Hormone Analogues to Prealbumin," Journal of the American Chemical Society, Vol. 104, No. 23: 64246434, 1982.

13. Wipff, G., A. Dearing, P.K. Weiner, J.M. Blaney, and P.A. Kollman, "Molecular Mechanics Studies of Enzyme-Substrate Interactions: The Interaction of L- and D-N-Acetyltryptophanamide with $\alpha$-Chymotrypsin," Journal of the American Chemical Society, Vol. 105, No. 4: 997-1005, 1983.

14. Weiner, S.J., P.A. Kollman, D.A. Case, U.C. Singh, C. Ghio, G. Alagona, S. Profeta, Jr., and P. Weiner, "A New Force Field for Molecular Mechanical Simulation of Nucleic Acids and Proteins," Journal of the American Chemical Society, Vol. 106, No. 3: 765-784, 1984.

15. Weiner, S.J., P.A. Kollman, D.T. Nguyen, and D.A. Case, "An all atom force field for simulations of proteins and nucleic acids," Journal of Computational Chemistry, Vol. 7, No. 2: 230-252, 1986.

16. Brooks, B.R., R.E. Bruccoleri, B.D. Olafson, D.J. States, S. Swaminathan, and M. Karplus, "CHARMM: A program for macromolecular energy, minimization, and dynamics calculations," Journal of Computational Chemistry, Vol. 4, No. 2: 187217, 1983.

17. Nilsson, L. and M. Karplus, "Empirical energy functions for energy minimization and dynamics of nucleic acids," Journal of Computational Chemistry, Vol. 7, No. 5: 591-616, 1986.

18. Hwang, M.J., T.P. Stockfish, and A.T. Hagler, "Derivation of Class II Force Fields. 2. Derivation and Characterization of a Class II Force Field, CFF93, for the Alkyl Functional Group and Alkane Molecules," Journal of the American Chemical Society, Vol. 116, No. 6: 2515-2525, 1994.

19. Sun, H., S.J. Mumby, J.R. Maple, and A.T. Hagler, "An ab Initio CFF93 AllAtom Force Field for Polycarbonates," Journal of the American Chemical Society, Vol. 116, No. 7: 2978-2987, 1994.

20. Wilson, E.B., Decius, J.C. and P.C. Cross, Molecular Vibrations: The Theory of Infrared and Raman Vibrational Spectra, Dover Publications, Mineola, New York, 1980.

21. Maple, J.R., U. Dinur, and A.T. Hagler, "Derivation of Force Fields for Molecular Mechanics and Dynamics from ab initio Energy Surfaces," Proceedings of the National Academy of Sciences of the United States of America, Vol. 85, No. 15: 5350-5354, 1988. 
22. Seymour, D.C., "ROCETS User's Manual," Vehicle \& Systems Development Department, Space Transportation Directorate, Marshall Space Flight Center, 1999.

23. REDTOP-2, Spaceworks Engineering, Inc., http://www.spaceworks.aero.

24. Van Wylen, G., R. Sonntag, and C. Borgnakke, Fundamentals of Classical Thermodynamics, John Wiley \& Sons, Inc. New York, New York, 1994.

25. Castka, J.F., H.C. Metcalfe, R.E. Davis, J.E. Williams, Modern Chemistry, Holt, Rinehart, and Winston, Austin, Texas, 2002.

26. Joback, K.G., "Cranium: Component Software for Physical Property Estimation”, Molecular Knowledge Systems, Inc., Bedford, New Hampshire, 1998.

27. Moran, M.J. and H.N. Shapiro, Fundamentals of Engineering Thermodynamics, John Wiley \& Sons, Inc. New York, New York, 2000.

28. Benson, S.W., Thermochemical Kinetics: Methods for the Estimation of Thermochemical Data and Rate Parameters, Second Edition, John Wiley \& Sons, Inc. New York, New York, 1976.

29. Benson, S.W. and J.H. Buss, "Additivity Rules for the Estimation of Molecular Properties, Thermodynamic Properties,” Journal of Chemical Physics, Vol. 29, Issue 3: 546-572, 1958.

30. Schmitt, R.J., J.C. Bottaro, M. Petrie, P.E. Penwell, "Synthesis of New HEDM: Extra High Energy Oxidizers and Fuels," AFRL-PR-ED-TR-1998-0023, September 2003.

31. Frenkel, D. and B. Smit, Understanding Molecular Simulation, From Algorithms to Applications, Academic Press. San Diego, California, 1996.

32. Loudon, G.M., Organic Chemistry, Fourth Edition, Oxford University Press. New York, New York, 2002.

33. Clark, J. "Intermolecular Bonding - van der Waals Forces," Online. May 20, 2005. Available: http://www.chemguide.co.uk/atoms/bonding/vdw.html.

34. Johnson, J.K., J.A. Zollweg, and K.E. Gubbins, "The Lennard-Jones equation of state revisited,” Journal of Molecular Physics, Vol. 78, No. 3: 591-618, 1993.

35. Cornell, W.D., P. Cieplak, C.I. Bayly, I.R. Gould, K.M. Merz, Jr., D.M. Ferguson, D.C. Spellmeyer, T. Fox, J.W. Caldwell, and P.A. Kollman, "A Second Generation Force Field for the Simulation of Proteins, Nucleic Acids, and 
Organic Molecules," Journal of the American Chemical Society, Vol. 117, No. 17: 5179-5197, 1995.

36. MacKerell, Jr., A.D., J. Wiórkiewicz-Kuczera, and M. Karplus, "An All-Atom Empirical Energy Function for the Simulation of Nucleic Acids," Journal of the American Chemical Society, Vol. 117, No. 48: 11946-11975, 1995.

37. Jorgensen, W.L, D.S. Maxwell, and J. Tirado-Rives, "Development and Testing of the OPLS All-Atom Force Field on Conformational Energetics and Properties of Organic Liquids," Journal of the American Chemical Society, Vol. 118, No. 45: 11225-11236, 1996.

38. Plimpton, S.J., "Fast Parallel Algorithms for Short-Range Molecular Dynamics," Journal of Computational Physics, Vol. 117: 1-19, 1995.

39. Huber, M.L., "NIST Thermophysical Properties of Hydrocarbon Mixtures Database (SUPERTRAPP) Version 3.1," NIST Supercritical Fluid Property Consortium and Standard Reference Data, Physical and Chemical Properties Division, Boulder, Colorado.

40. Lagache, M., P. Ungerer, A. Bloutin, and A.H. Fuchs, "Prediction of thermodynamic derivative properties of fluids by Monte Carlo simulation," Physical Chemistry Chemical Physics, Vol. 3: 4333-4339, 2001.

41. Cadena, C., Q. Zhao, R.Q. Snurr, and E.J. Maginn, "Molecular Modeling and Experimental Studies of the Thermodynamic and Transport Properties of Pyridinium-Based Ionic Liquids," Journal of Physical Chemistry B, Vol. 110: 2821-2832, 2006.

42. Cadena, C. and E.J. Maginn, "Molecular Simulation Study of Some Thermophysical and Transport Properties of Triazolium-Based Ionic Liquids," Journal of Physical Chemistry B, Vol. 110: 18026-18039, 2006.

43. Schmidt, M.W., K.K. Baldridge, J.A. Boatz, S.T. Elbert, M.S. Gordon, J.H. Jensen, S. Koseki, N. Matsunaga, K.A. Nguyen, S. Su, T.L. Windus, M. Dupuis, and J.A. Montgomery, "General Atomic and Molecular Electronic Structure System,” Journal of Computational Chemistry, Vol. 14: 1347-1363, 1993.

44. Hehre, W.J., R. Ditchfield, J.A. Pople, "Self-Consistent Molecular Orbital Methods. XII. Further Extensions of Gaussian-Type Basis Sets for Use in Molecular Orbital Studies of Organic Molecules," Journal of Chemical Physics, Vol. 56, No. 5: 2257-2261, 1972.

45. Levine, I.N., Quantum Chemistry, Fifth Edition, Prentice Hall. Upper Saddle River, New Jersey, 2000. 
46. Sun, H., J. Mumby, J.R. Maple, and A.T. Hagler, "Ab Initio Calculations on Small Molecule Analogues of Polycarbonates," Journal of Physical Chemistry, Vol. 99: 5873-5882, 1995.

47. Sherrill, C.D., Associate Professor of Chemistry \& Biochemistry, Georgia Tech School of Chemistry \& Biochemistry, Atlanta, GA. Personal Interview. May 3, 2006.

48. Scott, A.P. and L. Radom, "Harmonic Vibrational Frequencies: An Evaluation of Hartree-Fock, Møller-Plesset, Quadratic Configuration Interaction, Density Functional Theory, and Semiempirical Scale Factors," Journal of Physical Chemistry, Vol. 100: 16502-16513, 1996.

49. Hehre, W.J., Radom, L., Schleyer, P.v.R., and J.A. Pople, Ab Initio Molecular Orbital Theory, John Wiley \& Sons, Inc., New York, New York, 1986.

50. McBride, B.J., Zehe, M.J., and S. Gordon, "NASA Glenn Coefficients for Calculating Thermodynamic Properties of Individual Species," NASA-TP-2002211556, 2002.

51. Sun, H. and D. Rigby, "Polysiloxanes: ab initio force field and structural, conformational and thermophysical properties," Spectrochimica Acta Part A, 53: 1301-1323, 1997.

52. Rigby, D. and H. Sun, "Computer Simulations of Poly(ethylene oxide): Force Field, PVT Diagram and Cyclization Behaviour," Polymer International, 44: 311330, 1997.

53. Sun, H., Fried, J.R., and P. Ren, "The COMPASS force field: parameterization and validation for phosphazenes," Computational and Theoretical Polymer Science, 8: 229-246, 1998.

54. Allen, M.P. and D.J. Tildesley, Computer Simulation of Liquids, Oxford Science Publications, Oxford University Press, Oxford, England, 1987.

55. Sadus, R.J., Molecular Simulation of Fluids: Theory, Algorithms and ObjectOrientation, Elsevier Science B.V., Amsterdam, The Netherlands, 1999.

56. BreyFogle, F.W. III, Six Sigma, Second Edition - Implementing Six Sigma Smarter Solutions Using Statistical Methods, John Wiley \& Sons, Inc., Hoboken, New Jersey, 2003.

57. JMP - The Statistical Discovery Software, SAS Institute, http://www.jmp.com, October 2006. 
58. Hayter, A.J., Probability and Statistics For Engineers and Scientists, $2^{\text {nd }}$ Edition, Thompson Learning, Inc., Pacific Grove, California, 2002.

59. Ludovice, P.J., Associate Professor of Chemical \& Biomolecular Engineering, Georgia Tech School of Chemical \& Biomolecular Engineering, Atlanta, GA. Personal Interview. December 15, 2006.

60. van Wylen, G., R. Sonntag, and C. Borgnakke, Fundamentals of Classical Thermodynamics, John Wiley \& Sons, Inc., New York, New York, 1994.

61. Thomas, G.B. Jr. and R.L. Finney, Calculus and Analytic Geometry, $9^{\text {th }}$ Edition, Addison-Wesley Publishing Company, Reading, Massachusetts, 1996.

62. Denbigh, K., The Principles of Chemical Equilibrium, Fourth Edition, Cambridge University Press, Cambridge, England, 1981.

63. Black, W.Z. and J.G. Hartley, Thermodynamics, $3^{\text {rd }}$ Edition, HarperCollins College Publishers, New York, New York, 1996.

64. REDTOP-2, SpaceWorks Engineering, Inc., http://www.spaceworks.aero, December 2006.

65. Humble, R.W., Henry, G.N., and W.J. Larson, Space Propulsion Analysis and Design, McGraw-Hill Companies, Inc., New York, New York, 1995.

66. Chung, T., Ajlan, M., Lee, L.L., and K.E. Starling, "Generalized Multiparameter Correlation for Nonpolar and Polar Fluid Transport Properties," Industrial and Engineering Chemistry Research, Vol. 27: 671-679, 1988.

67. Chapman, S. and T.G. Cowling, The Mathematical Theory of Non-uniform Gases, Cambridge University Press, Cambridge, London, 1952.

68. Neufeld, P.D., Janzen, A.R., and R.A. Aziz, Journal of Chemical Physics, Vol. 57: 1100, 1972.

69. Pitzer, K.S., Journal of Chemical Physics, Vol. 7: 583-590, 1939.

70. Joback, K. G.; Reid, R. C. Estimation of Pure-Component Properties from GroupContributions. Chem. Eng. Comm. Vol. 57: 233-243, 1987.

71. MACH I, Inc., www.machichemicals.com, February 13, 2007.

72. Bleazard, J.G., "The Thermal Conductivity of Aqueous Electrolyte Solutions and Polar Liquids," Ph.D. Dissertation, Georgia Institute of Technology. Atlanta, Georgia, 1995. 
73. "Standard Test Method for Density and Relative Density of Viscous Materials by Lipkin Bicapillary Pycnometer," ASTM Standard D 1481-02, ASTM International, West Conshohocken, Pennsylvania, 2002.

74. "Standard Test Method for Kinematic Viscosity of Transparent and Opaque Liquids and the Calculation of Dynamic Viscosity," ASTM Standard D 445-04, ASTM International, West Conshohocken, Pennsylvania, 2004.

75. "Standard Specifications and Operating Instructions for Glass Capillary Kinematic Viscometers," ASTM Standard D 446-04, ASTM International, West Conshohocken, Pennsylvania, 2004.

76. "Standard Test Method for Determining Specific Heat Capacity by Differential Scanning Calorimetry," ASTM Standard E 1269-04, ASTM International, West Conshohocken, Pennsylvania, 2004.

77. Boyd, R.H., Sanwal, S.N., Shary-Tehrany, S, and D. McNally, "The thermochemistry, thermodynamic functions, and molecular structures of some cyclic hydrocarbons," Journal of Physical Chemistry, Vol. 75: 1264-1271, 1971.

78. Osmont, A and L. Catoire, "Evaluating Missile Fuels," Propellants, Explosives, Pyrotechnics, Vol 31, No. 5: 343-354, 2006.

79. McQuaid, M.J., "Computationally Based Design and Screening of HydrazineAlternative Hypergolic Fuels," Proceeding of the 23th Army Science Conference, Orlando, FL, 2002.

80. "NASA Exploration Systems Architecture Study Final Report," NASA-TM2005-214062, November 2005.

81. "Zero Boiloff Storage of Cryogenic Propellants Achieved at Lewis' Supplemental Multilayer Insulation Research Facility," NASA Glenn Research Center, http://www.grc.nasa.gov/WWW/RT1998/5000/5870plachta.html, January 2007.

82. Wertz, J.R. and W.J. Larson, Space Mission Analysis and Design, Microcosm Press, El Segundo, CA, 1999.

83. Humble, R.W., Henry, G.N., and W.J. Larson, Space Propulsion Analysis and Design, McGraw-Hill Companies, Inc., New York, New York, 1995.

84. Brauer, G.L., Cornick, D.E., Habeger, A.R., Petersen, F.M., and R. Stevenson, "Program to Optimize Simulated Trajectories (POST)," Final Report Martin Marietta Corporation, Denver, CO, 1975.

85. Rohrschneider, R.R., "Development of a Mass Estimating Relationship Database for Launch Vehicle Conceptual Design," Master's Project, School of Aerospace 
Engineering, Georgia Institute of Technology, 2002.

86. RL-10 Fact Sheet, Pratt \& Whitney Corporation, P\&W TS 9.01, 1999.

87. Gordon, S. and B.J. McBride, "Computer Program for Calculation of Complex Chemical Equilibrium Compositions and Applications I. Analysis," NASA-RP1311, 1994.

88. REDTOP, SpaceWorks Engineering, Inc., http://www.spaceworks.aero, January 2007.

89. Way, D. and J. Olds, "SCORES: Web-Based Rocket Propulsion Analysis for Space Transportation System Design," Space Systems Design Lab, Georgia Institute of Technology, Atlanta, Georgia. AIAA Paper 1999-2353, June 1999.

90. Dobbins, T.A., "Novel Alkynylsilane Compounds as Rocket Propellant Candidates," Organic Technologies, Coshocton, Ohio. AIAA Paper 2002-4347, July 2002. 


\section{VITA}

Timothy Salim Kokan was born on February 16, 1978 in Woodbridge, Virginia. He attended grade school at Elizabeth Vaughn Elementary, Woodbridge Middle, and Gar-Field High School in Woodbridge, Virginia. After graduating from Gar-Field High School in May of 1996, he enrolled at the University of Virginia. In May of 2000, he graduated with honors from UVA with a Bachelor of Science in Aerospace Engineering with a minor in Computer Science. The following fall he enrolled at the Georgia Institute of Technology to pursue graduate studies in Aerospace Engineering. Tim received his Master's Degree in Aerospace Engineering from Georgia Tech in May of 2002 and continued toward his Doctoral degree in the same field.

While at Georgia Tech, Tim spent his first year working with Dr. Amy Pritchett as a member of the Georgia Tech Reconfigurable Flight Simulator Lab. Here he studied modeling and simulation of aircraft and spacecraft. In August of 2001, Tim transferred to work with Dr. John Olds as a member of the Space Systems Design Lab. Here he studied reusable launch vehicle design with a concentration in propulsion and was a team leader for several advanced vehicle concepts. He also performed extensive research into highenergy-density matter propellants and their application to liquid rocket engines. During his graduate studies, Tim worked summers at Pratt \& Whitney Space Propulsion in West Palm Beach, Florida and the NASA Marshall Space Flight Center Propulsion Research Laboratory in Huntsville, Alabama.

Tim is a member of the American Institute of Aeronautics and Astronautics, and is a member of the Sigma Gamma Tau National Aerospace Honor Society. 